\title{
Inter-organisational Knowledge Transfer among Research and Development Organisations: Implications for Information and Communication Technology Support
}

\author{
Silke Retzer \\ A thesis submitted to Victoria University of Wellington \\ in fulfilment of the requirements for the degree of \\ Doctor of Philosophy \\ in Information Systems \\ School of Information Management \\ Te Whare Wananga o te Upoko o te Ika a Maui \\ Victoria University of Wellington \\ Wellington, New Zealand \\ 2010
}





\section{Abstract}

The purpose of this thesis was to investigate the nature of knowledge transfer among key organisations in the New Zealand (NZ) research and development (R\&D) sector. From these findings, practical implications for Information and Communication Technology (ICT) support were drawn.

Knowledge-intensive R\&D activities have seldom been investigated from a social network perspective. As Allen et al. (2007) point out there is a lack of research addressing the issue of knowledge transfer in inter-organisational social networks, specifically in the R\&D sector. By employing social network analysis (SNA), this study addressed this gap and analysed and identified inter-organisational knowledge transfer structures and processes among organisations by focussing on three cases. Key themes related to SNA included the identification of the network topology, informal key roles of organisations by their levels of interactivity in the networks and their types of relationships for transferring knowledge. The concepts underlying social network analysis played a significant role in informing this research.

Using a mixed method approach of case research and social network analysis, this thesis comprises an in-depth investigation into the nature of knowledge transfer among key R\&D organisations in the $\mathrm{NZ}$ environmental sector. The mixed methods approach employed followed three consecutive steps for each of the three cases. First, a qualitative phase was conducted. Data were collected from workshop/seminar participants. This helped to achieve a deeper understanding of the field under study, and the results were used to develop an online survey instrument for the second quantitative phase. In this quantitative phase the online survey was distributed to employees of various R\&D organisations. In total, 168 participants took part in this survey. Besides addressing SNA concepts, additional key themes covered by this survey were types of knowledge transferred, media used and preferred media characteristics as well as knowledge services for learning. In a final qualitative phase 
the workshop/seminar participants were asked follow-up questions in relation to key survey results with the aim of confirmation and explanation.

This research aimed to address practical and theoretical issues alike. From a practical perspective, this research provides important background information on how to increase collaboration among R\&D organisations in New Zealand. R\&D key players, such as Crown Research Institutes, private research organisations, universities, government departments, and industry organisations, need to collaborate more in order to increase commercialisation of research knowledge, reduce costs (e.g. reuse knowledge) and drive innovation.

From a theoretical perspective, this thesis adds knowledge to the application and further development of SNA concepts, such as tie strength, centrality and network structure, in an inter-organisational knowledge transfer context. Key findings were the definition of intermediate ties and their importance for knowledge transfer in inter-organisational social networks, recommendations of suitable centrality measures for knowledge networks and the identification of small-world networks in the R\&D sector.

Finally, implications for ICT support could be drawn. These implications may help increase inter-organisational collaboration. Possible ICT support was categorised into three levels that appeared useful in this study's knowledge transfer context: the individual level, the group level and the inter-organisational level. 


\section{Acknowledgements}

My sincere thanks are going to the following people who and organisations which have supported me on this $\mathrm{PhD}$ journey:

My primary supervisor, Prof. Pak Yoong, and my co-supervisors Dr.Val Hooper and Assoc. Prof. Hans Lehmann for making this such a memorable, inspiring and rewarding experience.

The people who and organisations which participated in my research. I appreciate their willingness to take part, and the time and effort they spent in doing so.

Markus, my partner, for his love, support and inspiration on this journey.

My family and friends in Germany, for always believing in my ability to achieve this milestone.

The members of the $\mathrm{PhD}$ study group at SIM, for always being supportive and interested.

Prof. Ronald Maier, Assoc. Prof. Hans Lehmann and Assoc. Prof. Julie Fisher for supporting me with their references to obtain a $\mathrm{PhD}$ scholarship.

Education New Zealand for providing financial support in the form of the New Zealand International Doctoral Research Scholarship (NZIDRS).

My German employer, msg systems, to grant me long-term leave for this $\mathrm{PhD}$.

The dedicated staff at SIM for their support.

Finally, I would like to thank my proof-readers Deborah Laurs and Jackie Bell for their great work in proof-reading my write-ups and the final $\mathrm{PhD}$ thesis.

Dedication

To my dear grandparents. 


\section{Table of Contents}

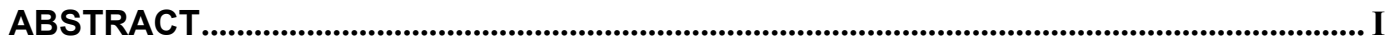

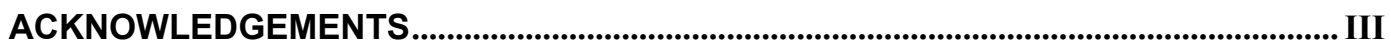

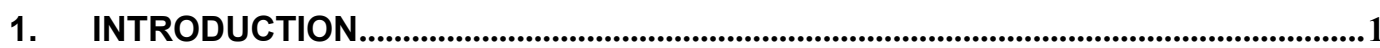

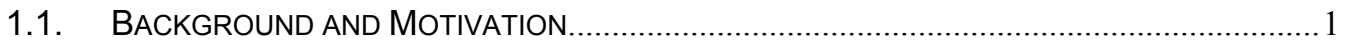

1.2. RESEARCH OBJECTIVES AND THE RESEARCH QUESTIONS ........................................ 4

1.3. RESEARCH DESIGN AND METHODOLOGY ............................................................... 5

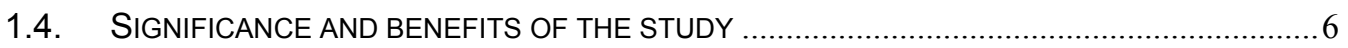

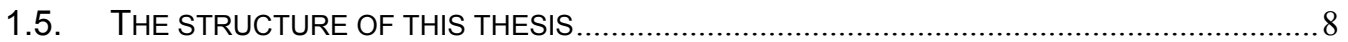

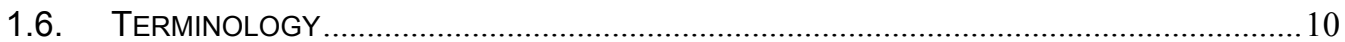

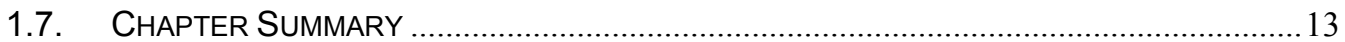

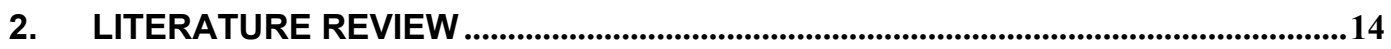

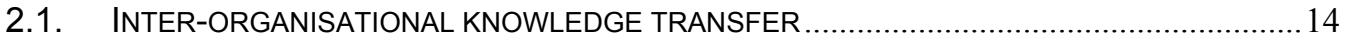

2.1.1 Definition of inter-organisational knowledge transfer .................................. 14

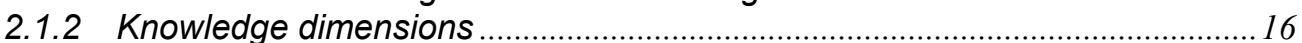

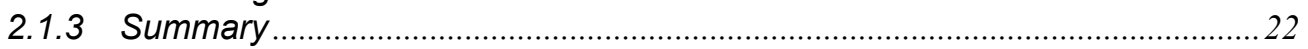

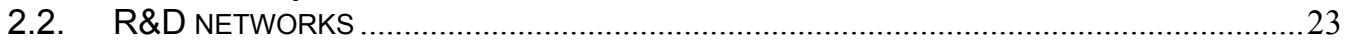

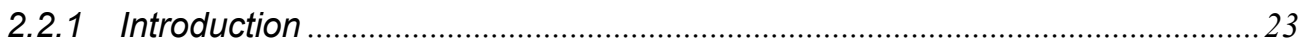

2.2.2 R\&D networks and inter-organisational knowledge transfer ............................24

2.2.3 The importance of informal knowledge transfer in R\&D networks.................. 30

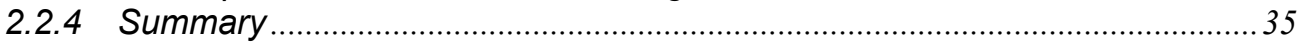

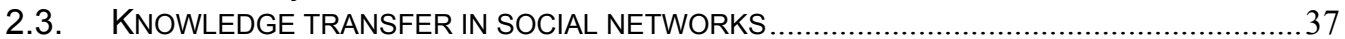

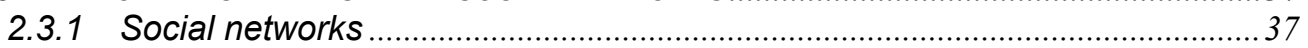

2.3.2 Knowledge transfer from a social network perspective ...................................... 37

2.3.3 Ontological network structures and knowledge transfer .................................40

2.3.4 Advice giving and seeking as key knowledge transfer processes in social networks

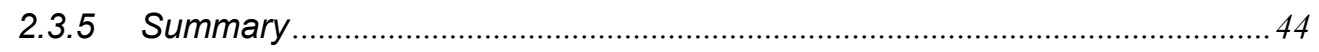

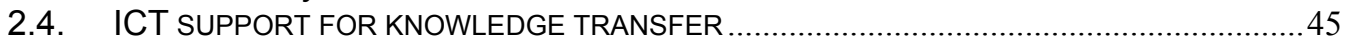

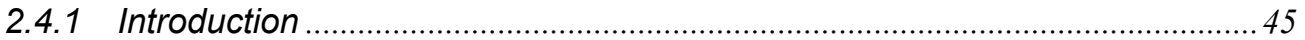

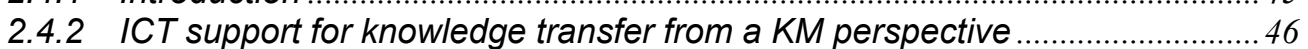

2.4.3 ICT support for knowledge transfer in social networks..................................52

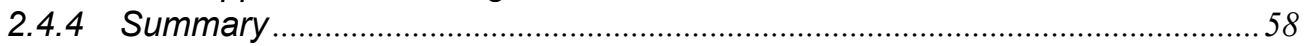

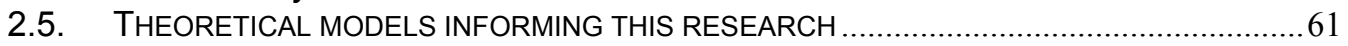

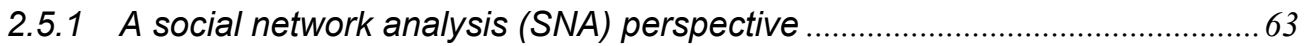

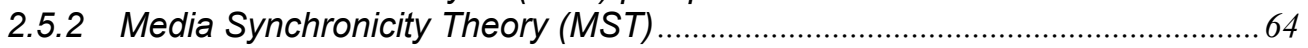

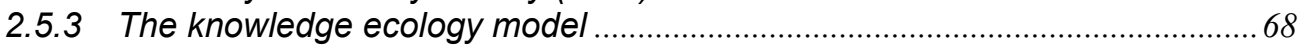

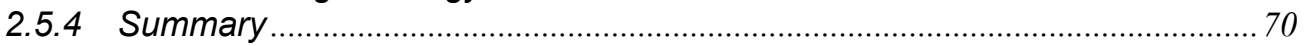

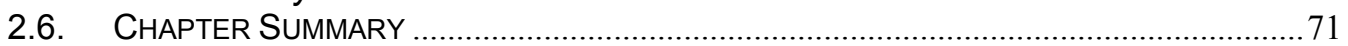

3. THE RESEARCH: PARADIGM, STRATEGY AND DESIGN .......................................74

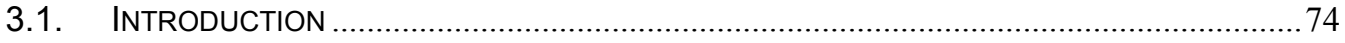

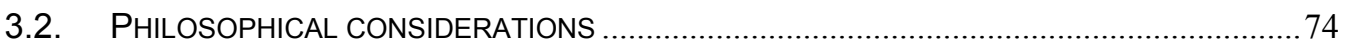

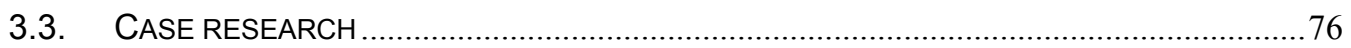

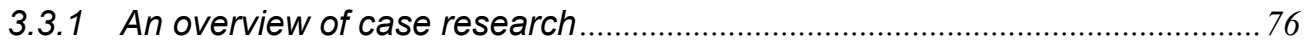

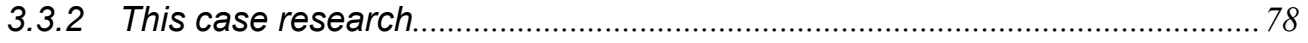

3.3.3 The unit of analysis .................................................................................... 81

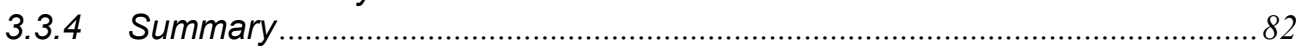

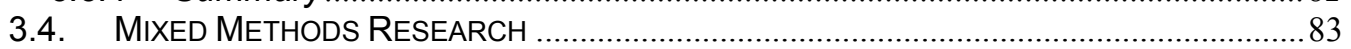

3.4.1 The nature of mixed methods research .................................................. 83

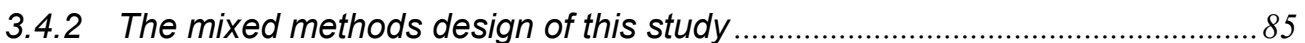

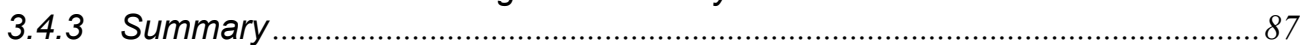

3.5. QUANTITATIVE RESEARCH WITH SOCIAL NETWORK ANALYSIS ....................................87

3.6. PHASE 1: QUALITATIVE DATA COLLECTION AND ANALYSIS PROCEDURES ..................97 
3.7. PhASE 2: QUANTITATIVE DATA COLLECTION FOR CONDUCTING SNA ........................102

3.8. PHASE 3: QUALITATIVE DATA COLLECTION AND ANALYSIS USING A WIKI TOOL..........108

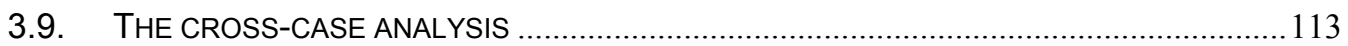

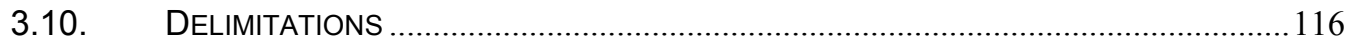

3.11. SUMMARY OF THE RESEARCH STRATEGY, DESIGN AND METHODS .......................116

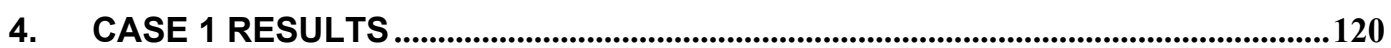

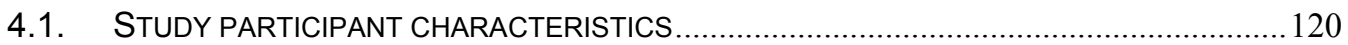

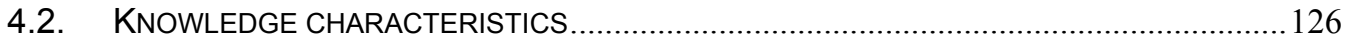

4.3. KEY FINDINGS RELATED TO STRUCTURAL PROPERTIES OF THE ADVICE NETWORK ....130

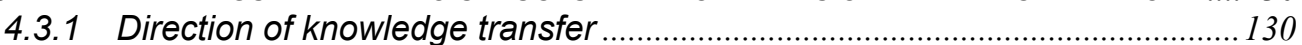

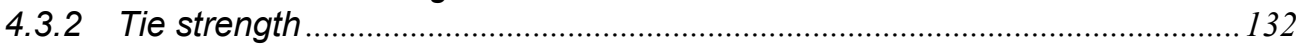

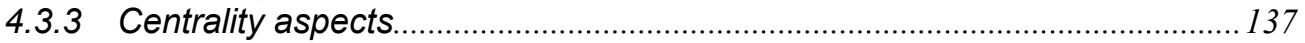

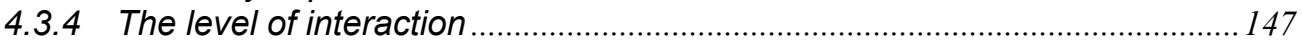

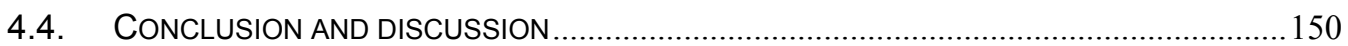

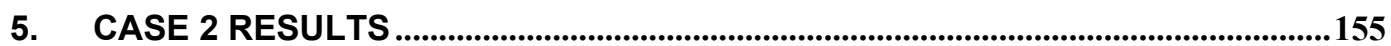

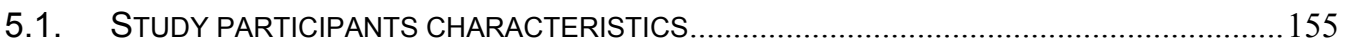

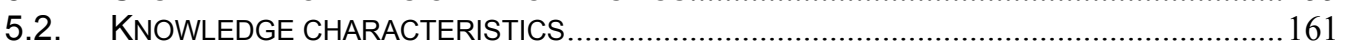

5.3. KEY FINDINGS RELATED TO STRUCTURAL PROPERTIES OF THE ADVICE NETWORK ....164

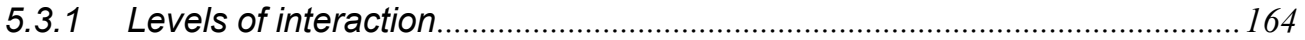

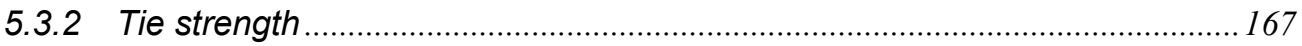

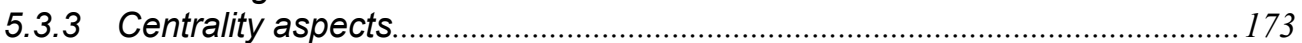

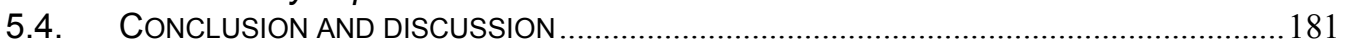

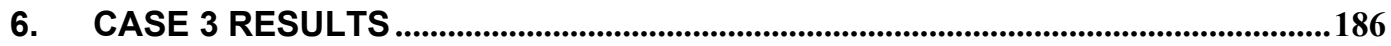

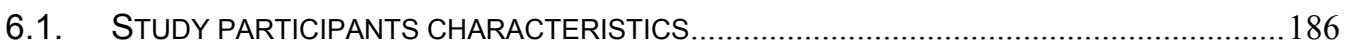

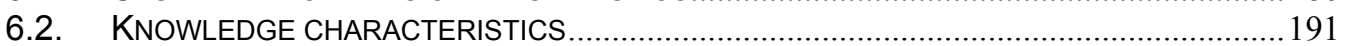

6.3. KEY FINDINGS RELATED TO STRUCTURAL PROPERTIES OF THE ADVICE NETWORK ....194

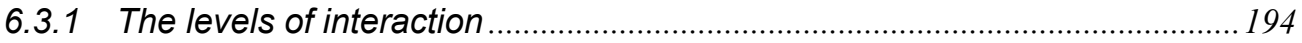

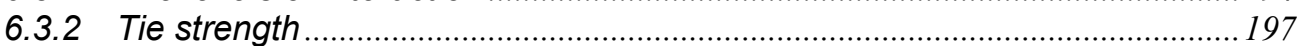

6.3.3 Centrality aspects.....................................................................................2. 200

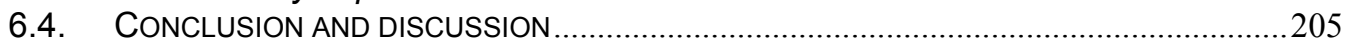

7. KEY RESULTS OF THE CROSS-CASE ANALYSIS: INTER-ORGANISATIONAL KNOWLEDGE TRANSFER IN SMALL-WORLD NETWORKS ..........................................210

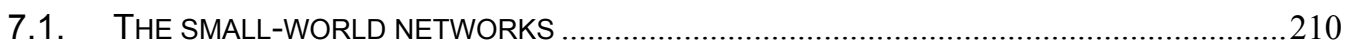

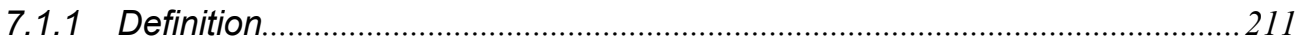

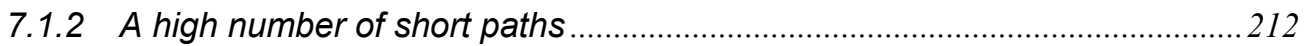

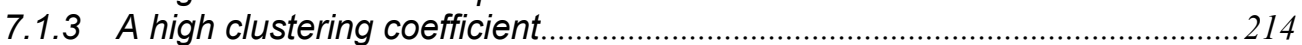

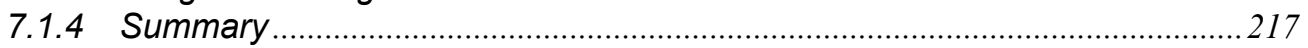

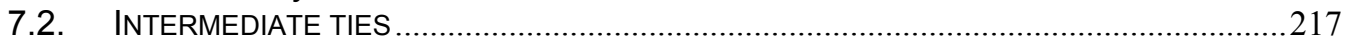

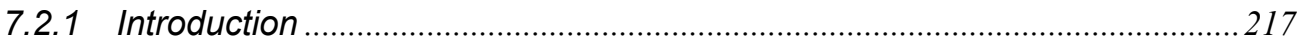

7.2.2 Criterion 1: $k$-means clustering for initial classification ...................................221

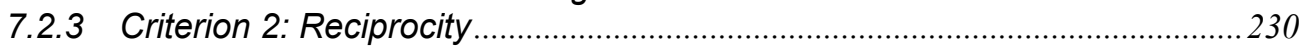

7.2.4 The important roles of intermediate ties .....................................................2235

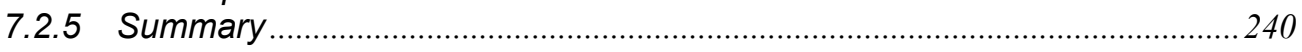

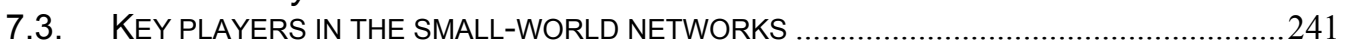

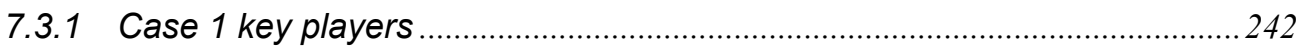

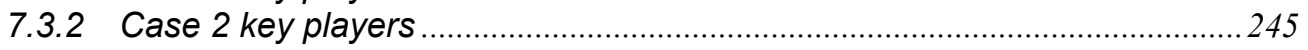

7.3.3 Case 3 key players .................................................................................2. 248

7.3.4 Most influential key players (Information Centrality scores) .........................250

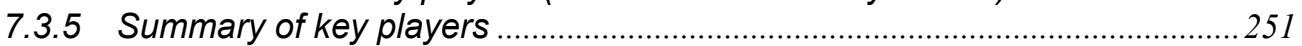

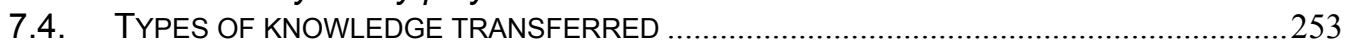

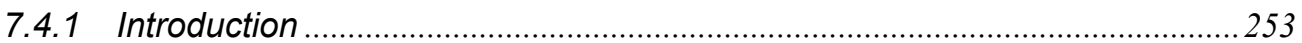

7.4.2 Electronically available types of knowledge ................................................2.255

7.4.3 Electronically unavailable types of knowledge ............................................2.256

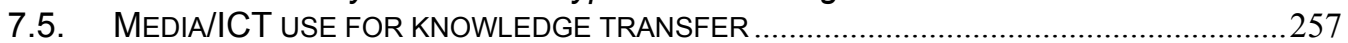

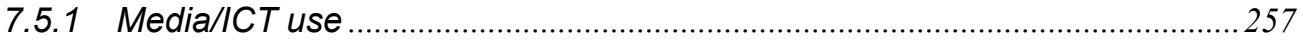

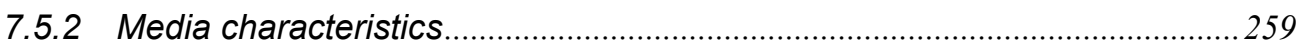




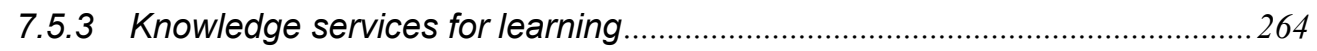

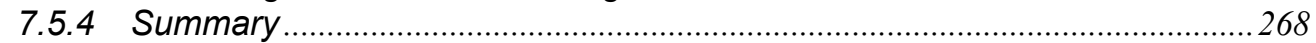

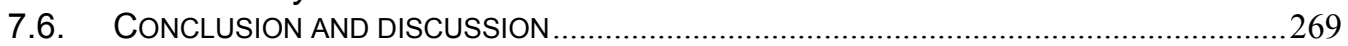

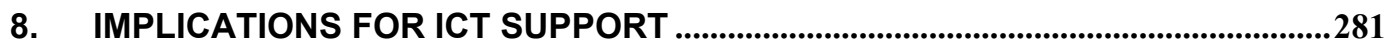

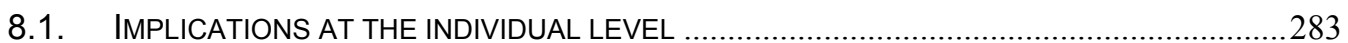

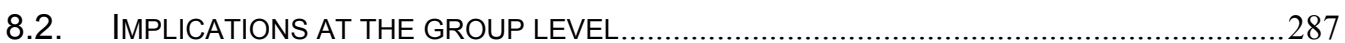

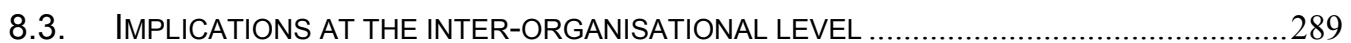

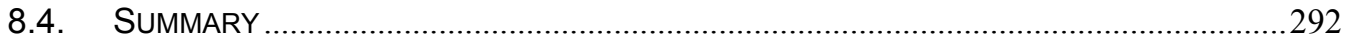

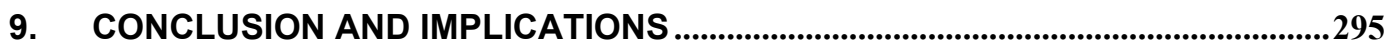

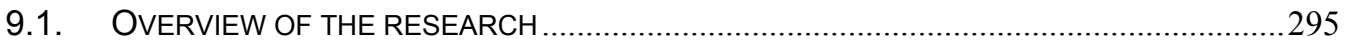

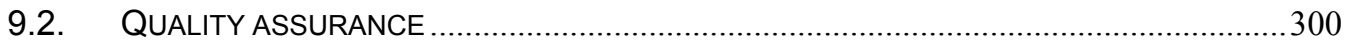

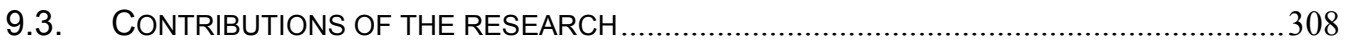

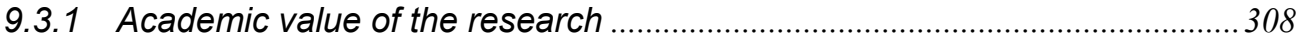

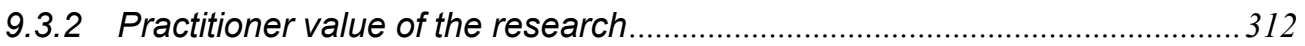

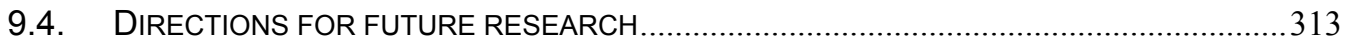

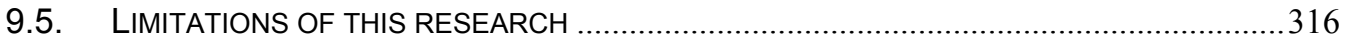

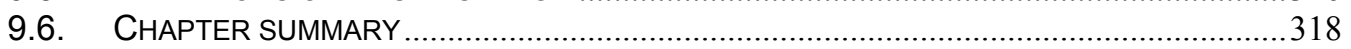

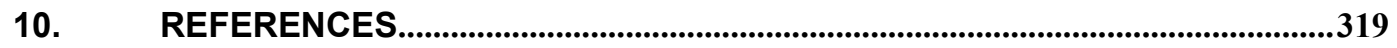

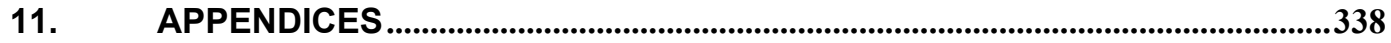




\section{List of figures}

Figure 1: Potential knowledge transfer around a research institute in a business network

Figure 2: Random network structure ........................................................ 41

Figure 3: Small-world network structure .................................................... 42

Figure 4: Knowledge transfer mechanisms (Despres \& Chauvel, 2000,

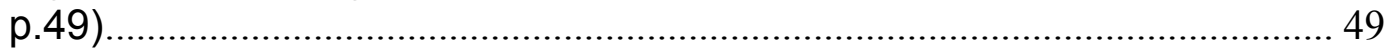

Figure 5: Multiple case study design ......................................................... 79

Figure 6: Decision tree for mixed methods design criteria for timing, weighting, and mixing (adapted from Creswell, 2007, p.80) ...................... 86

Figure 7: Components of data analysis: interactive model (Huberman \& Miles, 1998, p.181)

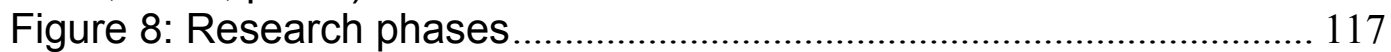

Figure 9: Research process outline ............................................................. 119

Figure 10: Job beginners (0-3 years) by gender in Case 1 ...................... 123

Figure 11: Study participants by job experience in Case 1 ..................... 123

Figure 12: Advice network of all organisations in Case 1 (whole network)

Figure 13: Weighted tie strength between key organisations .................. 134

Figure 14: Tie strength between groups of organisations in Case 1....... 136

Figure 15: Job beginners (0-3 years) by gender in Case 2 ..................... 158

Figure 16: Study participants by job experience in Case 2 ..................... 159

Figure 17: Case 2 advice network (whole network)................................... 168

Figure 18: Weighted tie strength between key (groups of) organisations in

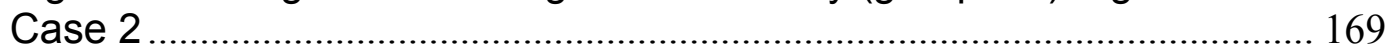

Figure 19: Tie strength between groups of organisations in Case 2....... 172

Figure 20: Job beginners (0-3 years) by gender in Case 3 .................... 187

Figure 21: Case 3 advice network (whole network)................................. 198

Figure 22: This study's three small-world networks in comparison.......... 214

Figure 23: Case 2 small-world network ..................................................... 233

Figure 24: This study's three small-world networks in comparison......... 237

Figure 25: : Role 2 example................................................................... 238

Figure 26: Small-world network 1: Matrix of key sinks and key sources. 244

Figure 27: Small-world network 2: Matrix of key sinks and key sources. 247

Figure 28: Small-world network 3: Matrix of key sinks and key sources. 249

Figure 29: Preferred ICT-based knowledge services 


\section{List of tables}

Table 1: Boisot's knowledge category model (McAdam \& McCreedy, 1999,

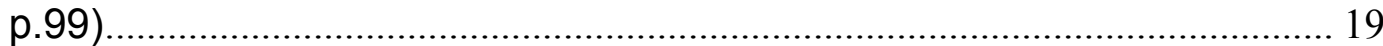

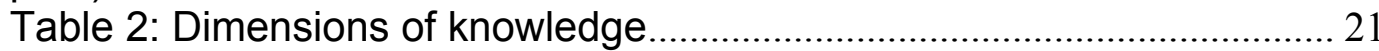

Table 3: Transfer effects associated with four combinations of knowledge complexity and tie strength (adapted from Hansen, 1999 p. 89)................ 38

Table 4: Knowledge transfer and ICT support ............................................ 51

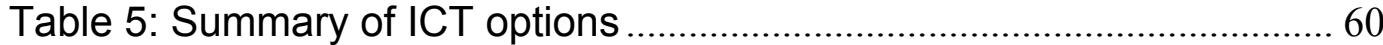

Table 6: Knowledge services (adapted from Wenger \& Ferguson, 2006, p.89).

Table 7: Overall numbers of study participants ......................................... 81

Table 8: Summary of methods used in Phase 1 .......................................... 98

Table 9: Key themes as a result of data analysis in Phase 1................... 101

Table 10: Coded examples of Phase 3 explaining/confirming key themes

Table 11: Key categories/findings of the cross-case analysis ................. 114

Table 12: Characteristics of key groups of organisations in Case 1....... 121

Table 13: No of study participants per organisational key type in Case 1

Table 14: Possible relationships between groups of organisations ........ 125

Table 15: Types of knowledge transferred compared between giving advice and seeking advice network in Case 1 ............................................ 128

Table 16: Types of knowledge and their flow characteristics................... 141

Table 17: Closeness centrality per group of organisation in Case 1........ 143

Table 18: Information centrality per group of organisation in Case 1 ...... 144

Table 19: Flow betweenness centrality scores per group of organisation in

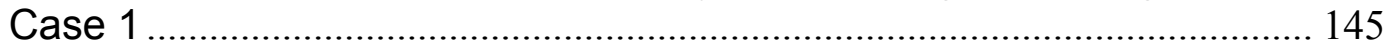

Table 20: Descriptive statistics for Case 1 ............................................... 147

Table 21: Summary of univariate statistics for key organisations by column

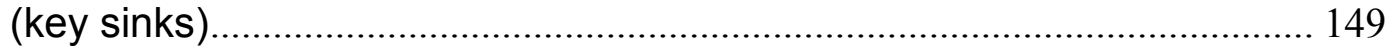

Table 22: Summary of univariate statistics for key organisations by row

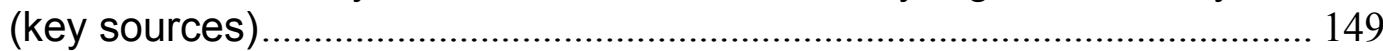

Table 23: Characteristics of key groups of organisations in Case 2 ....... 156

Table 24: No of study participants per key organisational type in Case 2

Table 25: Possible relationships between groups of organisation in Case 2

Table 26: Types of knowledge transferred in Case 2 .............................. 163

Table 27: Descriptive statistics of the Case 2 network.............................. 165

Table 28: Summary of univariate statistics for groups of organisations in

Case 2 by column (key sinks) ................................................................. 166

Table 29: Summary of univariate statistics for groups of organisations in

Case 2 by row (key sources) ..................................................................... 166

Table 30: Types of knowledge and their flow characteristics................... 175

Table 31: Closeness centrality per group of organisation in Case 2....... 177

Table 32: Case 2 Information centrality per group of organisation .......... 178

Table 33: Flow betweenness centrality scores per group of organisation in

Case 2 
Table 34: Study participants by job experience in Case 3

Table 35: No of study participants per organisational key type in Case 3

Table 36: Possible relationships between groups of organisation in Case 3

Table 37: Types of knowledge transferred in Case 3

Table 38: Descriptive statistics of the Case 3 network

Table 39: Summary of univariate statistics for groups of organisations in

Case 3 by column (key sinks) .

Table 40: Summary of univariate statistics for groups of organisations in

Case 3 by row (key sources) ................................................................... 196

Table 41: Types of knowledge and their flow characteristics.................. 200

Table 42: Closeness centrality per group of organisation in Case 3....... 202

Table 43: Information centrality per group of organisation in Case 3 ..... 203

Table 44: Flow betweenness centrality scores per group of organisation in

Case 3

Table 45: Clustering coefficients and network density ............................. 215

Table 46: Case 2 uni-directional (reciprocal) tie strength values.............. 219

Table 47: Values in relation to type of tie strength in Case 2................... 220

Table 48: Classification of total tie strength values for Case 1 ................. 225

Table 49: Initial cluster centres in Case 2 .................................................. 226

Table 50: Final cluster centres in Case 2 ............................................... 227

Table 51: Cluster memberships in Case 2 ................................................ 227

Table 52: Classification of total tie strength values for Case 2 ................ 228

Table 53: Classification of total tie strength values for Case 3 ................. 229

Table 54: Final classification of total tie strength values for Case 2 ........ 232

Table 55: Small-world network 1: Level of interaction by column (key

sinks)

Table 56: Small-world network 1: Level of interaction by row (key sources)

Table 57: Small-world network 2: Level of interaction by column (key sinks)

Table 58: Small-world network 2: Level of interaction by row (key sources)

Table 59: Small-world network 3: Level of interaction by column (key sinks)

Table 60: Small-world network 3: Level of interaction by row (key sources)

Table 61: Sum of Information Centrality scores per group of organisation

Table 62: Summary of key players in the three small-world networks ... 252 Table 63: Electronically available types of knowledge most important for transfer per case

Table 64: Electronically unavailable types of knowledge most important for

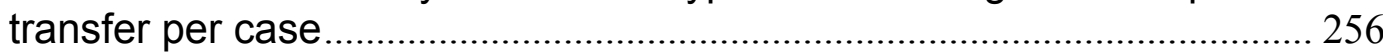

Table 65: Key study results that guided implications for ICT ...................... 282 Table 66: Mapping of knowledge service characteristics and ICT support (adapted from Wenger \& Ferguson, 2006) ............................................ 284 Table 67: Most preferred media characteristics ...................................... 285 Table 68: Summary: Levels of ICT support................................................ 294 


\section{List of appendices}

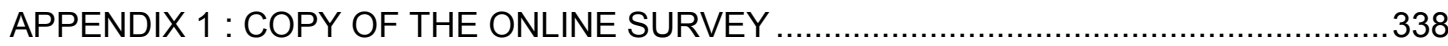

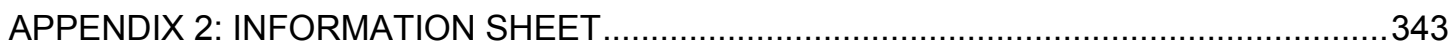

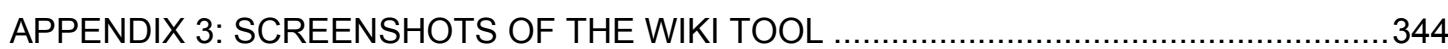

APPENDIX 4 : META-MATRIX OUTLINING A SET OF INITIAL CATEGORIES AS A

RESULT OF THE CROSS-CASE ANALYSIS.

APPENDIX 5 : CLUSTERED SUMMARY TABLE OUTLINING THE CLUSTERED CATEGORIES AS KEY CATEGORIES OF THE CROSS-CASE ANALYSIS. ......................347

APPENDIX 6: CASE 1 SURVEY RESULTS - BY GENDER ............................................348

APPENDIX 7: CASE 1 SURVEY RESULTS - TYPES OF KNOWLEDGE TRANSFERRED

APPENDIX 8: CASE 1 SURVEY RESULTS - CONTACT FREQUENCIES ……..................350

APPENDIX 9: CASE 1 SURVEY RESULTS - TIE STRENGTH MATRICES .........................354

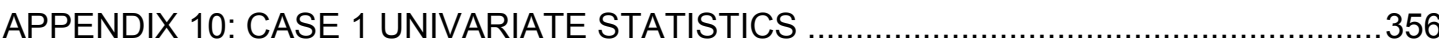

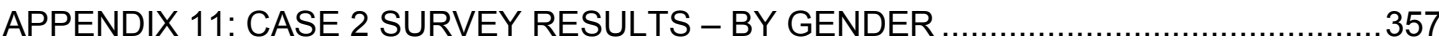

APPENDIX 12: CASE 2 SURVEY RESULTS - TYPES OF KNOWLEDGE

TRANSFERRED 358

APPENDIX 13: CASE 2 SURVEY RESULTS - TIE STRENGTH MATRICES ……...............359

APPENDIX 14: CASE 2 SURVEY RESULTS - CONTACT FREQUENCIES .........................360

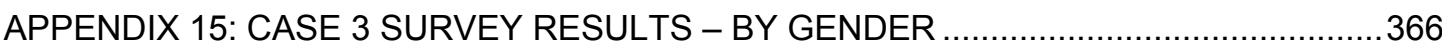

APPENDIX 16: CASE 3 SURVEY RESULTS - TYPES OF KNOWLEDGE

TRANSFERRED

APPENDIX 17: CASE 3 SURVEY RESULTS - TIE STRENGTH MATRICES …....................369

APPENDIX 18: CASE 3 SURVEY RESULTS - CONTACT FREQUENCIES …....................370

APPENDIX 19: CROSS-CASE ANALYSIS - DICHOTOMISED NETWORK DATA PER

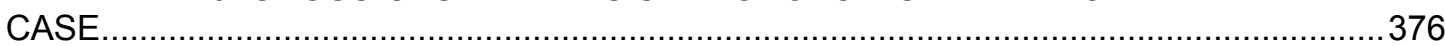

APPENDIX 20: CROSS-CASE ANALYSIS - INTERMEDIATE TIES DATA ….....................379

APPENDIX 21: CROSS-CASE ANALYSIS - NON-AGGREGATED INTERMEDIATE TIES DATA PER CASE

APPENDIX 22: CROSS CASE ANALYSIS - CASE 2 TIE STRENGTH DISTRIBUTION.....392 APPENDIX 23: CROSS-CASE ANALYSIS: UNIVARIATE STATISTICS RESULTS PER CASE AT THE GROUP OF ORGANISATION LEVEL

APPENDIX 24: CROSS-CASE ANALYSIS - INFORMATION CENTRALITY SCORES PER CASE

APPENDIX 25: CROSS-CASE ANALYSIS - PREFERRED TYPES OF KNOWLEDGE TRANSFERRED BY TIE STRENGTH IN CASE 2 AND CASE 3

APPENDIX 26: CASE 1 SURVEY RESULTS - MEDIA/ICT USE .....................................401

APPENDIX 27: CASE 2 SURVEY RESULTS - MEDIA/ICT USE ......................................402

APPENDIX 28: CASE 3 SURVEY RESULTS - MEDIA/ICT USE.......................................403

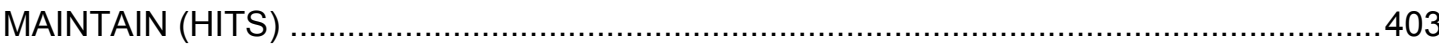

APPENDIX 29: CASE 1 SURVEY RESULTS - MEDIA/ICT USE BY TIE STRENGTH .......404

APPENDIX 30: CASE 2 SURVEY RESULTS - MEDIA/ICT USE BY TIE STRENGTH .......405 
APPENDIX 31: CASE 3 SURVEY RESULTS - MEDIA/ICT USE BY TIE STRENGTH .......406 APPENDIX 32: CASE 1 SURVEY RESULTS - PREFERRED MEDIA CHARACTERISTICS .407

APPENDIX 33: CASE 2 SURVEY RESULTS - PREFERRED MEDIA CHARACTERISTICS 409

APPENDIX 34: CASE 3 SURVEY RESULTS - PREFERRED MEDIA CHARACTERISTICS 411

APPENDIX 35: CASE 1 SURVEY RESULTS - PREFERRED KNOWLEDGE SERVICES .413 APPENDIX 36: CASE 2 SURVEY RESULTS - PREFERRED KNOWLEDGE SERVICES .414 APPENDIX 37: CASE 2 SURVEY RESULTS - PREFERRED LEARNING METHOD CHARACTERISTICS 415

APPENDIX 38: CASE 3 SURVEY RESULTS - PREFERRED KNOWLEDGE SERVICES .416 APPENDIX 39: CASE 3 SURVEY RESULTS - PREFERRED LEARNING METHOD CHARACTERISTICS 417 APPENDIX 40: INTERVIEW GUIDELINES 


\section{Introduction}

\subsection{Background and Motivation}

\section{Background}

The purpose of this study was underpinned by three key issues associated with the recognition that improvements are required in the New Zealand research and development (R\&D) sector. Firstly, there is a need for increased collaboration among $R \& D$ organisations. The results of an R\&D survey conducted by the Ministry of Research, Science and Technology (MORST) and Business NZ in 2006 highlight the importance for more collaboration among R\&D organisations (Business NZ, 2006) Examples from international businesses constantly demonstrate the benefits of collaboration between, for example, public sector organisations and other institutes. Furthermore, "in a small country such as New Zealand where a number of barriers of scale exist, collaboration across a variety of organisations is critical to keep research and development costs down and to increase the chances of successful innovation" (Business NZ, 2006).

Secondly, R\&D organisations are expected to source more non-governmental funding. MORST's latest annual report for 2006, Research and development in New Zealand: A decade in review, compares New Zealand's R\&D expenditure patterns with other members of the Organisation for Economic Co-operation and Development (OECD) and found that New Zealand's R\&D expenditure is still "only about one-third of the OECD average" (MORST, 2006, p.18). In order to raise New Zealand's R\&D investment to at least the OECD average, Porter's (2001) study on New Zealand's competitiveness suggested an increase of non-governmental R\&D funding. A key approach for research institutes in achieving this appears to be commercialisation, for example in the form of transferring critical R\&D knowledge to potential commercial users (Roberts, 1988). 
Finally, the drive for increased non-governmental $R \& D$ funding seems to be particularly significant for Crown Research Institutes (CRIs), as their governmental funding has continuously decreased in recent years (MORST, 2006). Therefore, the CRIs need to find different and innovative approaches in sourcing those nongovernmental funds.

Consequently, an improvement in collaboration and knowledge transfer among R\&D key players and potential stakeholders is required to overcome these key issues. Improved collaboration can stimulate interest in $R \& D$ activities, leading to increased commercialisation. An R\&D network consisting of $R \& D$ institutes, government agencies, universities and industry players, appears to provide the necessary environment for this collaboration.

\section{Theoretical Motivation}

Previous research into the nature of collaboration in R\&D networks provides some insights into the characteristics of informal relations between scientific and technical employees (Allen, James \& Gamlen, 2007). Employees working in R\&D tend to build very strong trust relationships with their colleagues. Thus, they turn to these relationships for assistance and advice and do not consider alternative sources, such as other personnel or data sources (Allen, 1977).

Therefore, these informal social relationships should be considered in order to improve and increase collaboration between R\&D firms. Compared to "formal channels of communication which rarely accurately reflect the working relationships between individuals" (Cross, Borgatti \& Parker, 2002a cited in Allen et al., 2007, p.180), informal relationships are the ones which really encourage the exchange of knowledge. Informal networks are crucial to the work of scientific and technical employees, as they can cross organisational and geographical boundaries. They also create the ability for the firm to innovate (Cross \& Parker, 2004). Thus, it is very important to understand how informal networks within R\&D are formed, how they are structured and how they work (Cross, Nohria \& Parker, 2002b). 
Informal social relationships have typically been investigated by using concepts of social network analysis. A social network comprises a set of social entities, such as individuals, groups or organisations, which are linked to each other in order to exchange, for example, information (Haythornthwaite, 1999). In regard to interorganisational knowledge transfer, the social relationship can be investigated at the inter-organisational level. Social network research focuses on relationships between these entities, which can be represented by "communications among members of a group" (Wasserman \& Faust, 1994, p.i) and "informal, interpersonal relations in social systems" (Scott, 2000, p.7). A social network perspective appeared to be an appropriate way of investigating knowledge transfer in these networks (Cross, Laseter, Parker \& Velasquez, 2006; Hansen, 1999). Moreover, this perspective has been applied previously to the knowledge management context and is suitable for the identification of social structures and knowledge transfer activities in a network (Anklam, 2002; Chan \& Liebowitz, 2006).

Knowledge transfer in these groups can be facilitated by different means that may include ICT. Besides the need for appropriate ICT systems to support knowledge transfer in locally distributed work environments (Pauleen \& Yoong, 2001), the appropriate storage and reuse of knowledge appears significant.

ICT for knowledge transfer is ideally part of an organisation-wide knowledge management strategy (Hansen, Nohria \& Tierney, 1999). An organisation's knowledge management strategy determines whether it views knowledge as largely stored in computers, or as residing in people. Organisations that rely on tacit knowledge and create and transfer highly complex knowledge, for example R\&D institutes, may follow a strategy that supports knowledge transfer between people. This strategy may also affect the technologies chosen to support this knowledge transfer. Moreover, in terms of an inter-organisational environment, the individual $\mathrm{KM}$ strategies and technologies of organisations participating in a network need to converge and represent together the network's KM goals. 
Furthermore, user preferences appear significant according to the effective use of ICT for knowledge transfer. User acceptance and motivation to use ICT seems to be significantly dependent on the additional value ICT provides to potential users. Therefore, ICT support for knowledge transfer in an inter-organisational context is also dependent on user preferences.

In academic literature, there is a history of investigating knowledge transfer, its success factors and its barriers, particularly in formal collaboration, for example in strategic alliances (e.g. Mowery, Oxley \& Silverman, 1996; Rolland \& Chauvel, 2000; Simonin, 1999; Zander \& Kogut, 1995). ICT has been suggested to support various stages of knowledge transfer (e.g. Holsapple \& Jones, 2004; Kwan \& Cheung, 2006). Nevertheless, there is a lack of research addressing the issue of informal knowledge transfer in inter-organisational social networks, specifically in the R\&D sector. As Allen et al. (2007) point out: "Research on knowledge-intensive R\&D activities with a social network perspective remains relatively rare" (p.179). In addition, the concepts of social network analysis (SNA) are still relatively young and therefore under constant development. Research into this area may help to add methodological knowledge. There is also a gap in research addressing the use of ICT to support social relations and their knowledge transfer in inter-organisational networks.

\subsection{Research Objectives and the Research Questions}

This study aims to gain a better understanding of how people who are working in the R\&D sector transfer knowledge within social networks. In regard to interorganisational knowledge transfer, the social relationship is investigated at the interorganisational level. In particular, this study looks at diverse groups of organisation, such as local government, universities and Crown Research Institutes, that are communicating with each other. Other key aspects are the identification of the

preferred types of knowledge and the media used for the transfer. In addition, this 
study aims to draw implications for ICT that may support these forms of knowledge transfer. Therefore, a number of propositions in the form of practical recommendations for ICT support are developed. For this purpose, knowledge transfer structures are linked to suitable supportive ICT while bearing in mind the context of this study. In addition to the inter-organisational level, these implications for ICT support are drawn at the individual and group level to address specific supportive tools for knowledge transfer among individuals and within groups as part of the larger inter-organisational R\&D networks.

This research is guided by the following research question:

What are the knowledge transfer structures and processes among organisations within an R\&D network?

This question consists of three sub-questions:

1) What are the key structural properties of advice networks and their implications for knowledge transfer?

2) Which types of knowledge are transferred in the advice networks?

3) How can ICT support these knowledge transfer structures and processes?

\subsection{Research Design and Methodology}

A mixed methods approach using qualitative and quantitative methods is employed to answer the three research sub-questions. This approach allows for an investigation of a phenomenon from diverse perspectives (Creswell, 2003). The researcher is able to develop a better understanding of the research context and therefore tends to produce better results. Case research is the leading research strategy. SNA is embedded in this strategy. The philosophical stance of this research is based on a combination of 
interpretive and positivist perspectives. This mixed methods approach uses complementary methods to gain a better understanding of the nature of knowledge transfer among R\&D organisations and will help answer the first two research subquestions. The information about the nature of this knowledge transfer provides a solid background to identify how ICT can facilitate this knowledge transfer to answer the third research sub-question.

A sequential approach is employed that follows three consecutive steps for each of the three cases. First, a qualitative phase of participant observation and interviews is undertaken. Data collection in this phase is conducted with workshop/seminar participants. This helps to gain a deeper understanding of the field under study. The results are used to develop an online survey instrument for the second phase. In this quantitative phase the online survey is distributed to employees of various R\&D organisations including previous workshop/seminar participants. The survey addresses SNA concepts and other key themes such as types of knowledge transferred, media used and preferred media characteristics as well as knowledge services for learning. In a final qualitative phase the study workshop/seminar participants are asked follow-up questions in order to confirm and explain key results of the survey. This study's design and methodology focuses on the investigation of knowledge transfer structures (with SNA) which are embedded in the key knowledge transfer processes of advice giving and seeking activities in the workplace.

\subsection{Significance and benefits of the study}

This study provides a number of significant contributions and implications for both academics and practitioners. The main areas are outlined below. These aspects will be expanded upon in detail in Chapter 8. 
Firstly, from an academic perspective, it addresses an acknowledged gap and emerging key theme in knowledge management. Successful knowledge transfer has been seen as vital for organisational versatility, innovation, and competitiveness. In the context of dynamic industrial co-operation such as is seen in the R\&D sector, successful inter-organisational knowledge transfer, together with existing social relations, plays a key role for organisations. Yet there has been almost no research into how informal knowledge transfer among R\&D organisations occurs (Allen et al., 2007). In order to identify potential for improvement this study explores knowledge transfer in social networks, which comprise members of various organisations.

In addition, social relations and organisational boundaries have been identified as two of six key emerging themes in the knowledge management discipline into which further research is required (Argote, McEvily \& Reagans, 2003). This study helps to address this gap in the research literature.

Secondly, the concepts of social network analysis are still young and therefore under constant development. Research into this area adds methodological knowledge to current SNA literature.

Thirdly, this study enriches research into ICT for collaborative inter-organisational knowledge transfer. This thesis aims to extend research into social or collaborative ICT (such as forums, chat and online diaries). This is a relatively new area of research, especially in relation to how these ICT can facilitate knowledge transfer between organisations. This should benefit practitioners and researchers alike.

Finally, from a practitioner perspective, this research aims to provide recommendations for suitable ICT support to enhance inter-organisational learning environments. In addition, managerial implications are discussed. 


\subsection{The structure of this thesis}

This section provides a brief outline of each of the remaining eight chapters of this thesis.

\section{Chapter 2: Literature review}

The literature review covers the definition of inter-organisational knowledge transfer in the context of this study. Further, the context of R\&D networks and the strategic importance of knowledge transfer within these networks are outlined. Moreover, important types of knowledge and knowledge transfer models from a knowledge management perspective are outlined and summarised. This particularly considers the informal knowledge transfer process of advice giving and seeking activities. The significance of workplace learning, especially inter-organisational learning in the workplace, is addressed. In addition, literature about knowledge transfer in social networks is investigated, and possible ICT to support inter-organisational knowledge transfer is introduced. Finally, informing theoretical concepts, the social network perspective, Media Synchronicity Theory (Dennis, Fuller \& Valacich, 2008) and Wenger and Ferguson's (2006) framework for knowledge services, are described.

\section{Chapter 3: The Research: Paradigm, Strategy and Design}

This chapter describes the selected research method and its practical execution. The philosophical and methodological approach of this study is outlined, including case research as the leading research strategy, which embeds SNA for measuring social network data. The case research includes a multiple case study of three cases. The mixed methods study design is explained and the methods for data collection and analysis are described. Moreover, the key concepts of SNA are explained in detail. 


\section{Chapter 4: Case 1 results}

This chapter describes the key results of the first of the three case studies. First, study participant characteristics are outlined. Key findings include types of knowledge transferred in the social network of Case 1 and its structural key properties. For Case 1 , the research sub-questions one and two are answered.

\section{Chapter 5: Case 2 results}

This chapter describes the key results of the second case study. First, study participant characteristics are outlined. Key findings include types of knowledge transferred and structural key properties of the network in Case 2. The research subquestions one and two can be answered for this case.

\section{Chapter 6: Case 3 results}

This chapter describes the key results of the third case study. As in Cases 1 and 2, key findings include types of knowledge transferred and structural key properties of the network in Case 3. The research sub-questions one and two can be answered for this case.

\section{Chapter 7: Key results of the cross-case analysis: inter-organisational knowledge} transfer in small-world networks

This chapter presents key results of the cross-case analysis of the three cases. The first part focuses on the application of social network analysis to the knowledge management context and outlines key findings in regard to the networks' structural properties. The second part describes key results in regard to study participants'

media/ICT use for knowledge transfer, their preferred media characteristics and knowledge services for learning. This chapter finally allows the research to fully answer the research sub-questions one and two. 


\section{Chapter 8: Implications for ICT support}

This chapter presents the practical recommendations for ICT support building upon key results of the cross-case analysis. These recommendations are presented in the form of propositions which help answer the third research sub-question.

\section{Chapter 9: Conclusion and Implications}

Finally, the conclusion and implications chapter summarises theoretical and practical contributions and outlines quality assurance aspects. Additional topics for further research are discussed.

\subsection{Terminology}

\section{Knowledge transfer}

Knowledge transfer in general examines the shift of knowledge from one knowledge domain to other specialised knowledge domains (Carlile \& Rebentisch, 2003). Interorganisational knowledge transfer, in the context of this research, describes the movement of domain knowledge between two or more organisations in a knowledgeintensive environment, such as the R\&D sector. This knowledge transfer is informal in nature, and it can show various levels of interaction dependent on the degree of unidirectional and reciprocal transfer. Inter-organisational knowledge transfer at the workplace further includes processes, such as advice giving and seeking, and structures which are dependent on the social structures among actors of the knowledge transfer network. 


\section{Small-world network}

A small-world network has a decentralised structure. Small-world networks include some degree of centralisation and thus do not have the same degree of decentralisation as random networks (Kwon, Oh \& Jeon, 2007). They also have a high number of direct connections between any two network members (Albert \& Barabasi, 2000). Small-world networks are typical in many large-scale real-world networks such as biological networks, the Internet or social networks (Nguyen, 2006).

\section{Relationship/link/connection}

In social network terminology, which is used throughout this thesis, a relationship is a specific kind of interaction between actors in a social network. It can be defined by the type of resource exchanged between these actors. The resource exchanged is referred to as the content of the relationship. In this thesis, this content is knowledge. Knowledge transferred between actors describes the interactions. The terms link or connection can be used instead of relationship.

\section{Tie}

A tie is the set of one or more specific interactions that connect actors (Haythornthwaite, 1996). Thus a tie describes a relationship with a certain set of characteristics between actors in a network (Hanneman, 2005). A tie is a term often used in social network analysis terminology. In particular, the terms weak tie and strong tie are often used. They describe either a relationship with less contact (weak tie) or a relationship with regular contact (strong tie), whereas the contact frequency represents only one indicator of tie strength besides others such reciprocity and intimacy. 


\section{Intermediate tie}

Besides weak and strong ties, intermediate ties are defined as medium-strong relationships. This level of tie strength may again be based on several indicators, for example contact frequency and reciprocity. In addition, the inter-organisational context of this study also considers the number of study participants from each organisation when measuring tie strength.

\section{Key sink/key source}

A key sink describes an organisation with a high level of receiving relationships. In contrast, such an organisation does not initiate a lot of relationships (Hanneman, 2005). Key sinks represent knowledge givers and therefore leaders in their knowledge domain in this study. Individual organisations may also be key sources of relationships. These organisations initiate a lot of relationships, but do not receive many relationships. Key sources represent knowledge seekers in this study's context.

\section{Key knowledge broker}

In this study, a key knowledge broker is both a key source and a key sink. Therefore, an organisation which represents a key knowledge broker receives and initiates a lot of relationships and has a high level of interactivity in regard to knowledge transfer (give and seek) in the network. 


\subsection{Chapter Summary}

The topic of inter-organisational knowledge transfer among R\&D organisations was introduced, the research objectives were identified and the research questions developed. The research methodology was outlined, and the potential value of the research in the academic and practitioner fields alike were highlighted. Finally, the structure of the thesis chapters was presented. 


\section{Literature Review}

The literature review provides an overview of the most relevant literature concerned with inter-organisational knowledge transfer in knowledge-intensive environments such as the R\&D sector. The review firstly covers an introduction to interorganisational knowledge transfer and current distinctions among types of knowledge in the knowledge management context. Then, R\&D networks are defined, and an

outline of literature investigating knowledge transfer in R\&D networks is given. This outline particularly highlights the importance of informal knowledge transfer. Consideration is also given to the linkage between knowledge transfer and workplace learning. Further, the role of social networks in knowledge transfer is explored. Possible ICT support for inter-organisational knowledge transfer in social networks is described. This includes two conceptual models that recommend certain types of ICT for knowledge transfer. In particular, the individual, group and inter-organisational levels are investigated for ICT support. Finally, the theoretical frameworks informing this research are described.

\subsection{Inter-organisational knowledge transfer}

In the first section, inter-organisational knowledge transfer is defined. Then, various types of knowledge are described. This includes an outline of how the concept of knowledge and the concept of commercialisation may be linked. Further, the importance of tacit and explicit knowledge is explored.

\subsubsection{Definition of inter-organisational knowledge transfer}

Knowledge transfer in general examines the shift of knowledge from one knowledge domain to other specialised knowledge domains (Carlile \& Rebentisch, 2003). Interorganisational knowledge transfer in particular can be defined as "an event through 
which one organisation learns from the experience of another" (Easterby-Smith, Lyles \& Tsang, 2008, p.677). In this regard, inter-organisational knowledge transfer involves at least two organisations. This aspect suggests meaningful perspectives to explore the interactive dynamics between these organisations (Easterby-Smith et al., 2008).

Recent studies that investigated the benefits of knowledge transfer in interorganisational environments considered formal as well as informal communication structures (e.g. Harryson, Dudkowski \& Stern, 2008; Pérez-Nordtvedt, Kedia, Datta \& Rasheed, 2008; Sammarra \& Biggiero, 2008). In addition, van Wijk, Jansen and Lyles (2008) recognised that previous research has argued that social relations among actors play an important role in facilitating knowledge transfer (Adler \& Kwon, 2002). Investigations of formal structures included the transfer between alliance partners (Becerra, Lunnan \& Huemer, 2008), supplier networks (Mason \& Leek, 2008) and innovation networks (Harryson et al., 2008; Sammarra \& Biggiero, 2008).

Inter-organisational knowledge transfer, in the context of this research, describes the movement of domain knowledge between two or more organisations in a knowledgeintensive environment, such as the R\&D sector. This knowledge transfer is informal in nature, and it can show various levels of interaction dependent on its degree of reciprocity. Reciprocity describes bi-directional knowledge transfer. The nature of inter-organisational knowledge transfer is further elaborated throughout this study. In particular, in Sections 4.3., 5.3. and 6.3. as well as Sections 7.1., 7.2. and 7.3. the structural properties of networks that underlie this knowledge transfer are described. This includes levels of interaction and direction and strength of knowledge transfer that further explain knowledge transfer in this study's context.

The following section describes various knowledge dimensions that are outlined in literature. 


\subsubsection{Knowledge dimensions}

This section on knowledge dimensions outlines knowledge as a part of commercialisation. It also explores tacit and explicit knowledge characteristics and then presents a range of alternative knowledge types described in the knowledge management literature.

\section{Knowledge in the context of commercialising innovation}

The nature of knowledge can be described by metaphors which, for example, view knowledge as an object, an asset, and as situated practice (Schultze \& Leidner, 2002). These differentiations are based on how knowledge can be operationalised. For example, the perspective that views knowledge as an object, which is independent from an individual, refers to rules, chunks, explanations and problem-solution sets as knowledge (Schultze \& Leidner, 2002). "These operationalisations are closely associated with problem-solving tasks in research on knowledge-based systems" (Schultze \& Leidner, 2002, p.221).

In contrast, other perspectives describe knowledge residing within the individual. This knowledge can be described as an asset comprising individual expertise, competence and job experience (Schultze \& Leidner, 2002). Moreover, knowledge as

situated practice describes knowledge being socially constructed and shared among people who are working in the same professional field.

According to Rogers (2003), knowledge is something that 'occurs', which might, for example, be the result of a knowledge creation or knowledge transfer process. Knowledge generation, which includes knowledge creation as a main component, and knowledge application, which includes knowledge transfer as a main component, are represented as the two dimensions of KM (Despres \& Chauvel, 2000). However, this "knowledge occurs when an individual (or other decision-making unit) is exposed to an innovation's existence and gains an understanding of how it functions" (Rogers, 2003, p.169). 
Knowledge arising from practice when applying innovations can be commercialised (Rogers, 2003). Rogers (2003) describes innovations as ideas derived from research that lead users in practice, who focus on problem-solving when applying an innovation. This definition of innovation may relate to different types of knowledge. For example, problem-solving solutions for the application of an innovation in a specific context may refer to knowledge as an object, particularly if this knowledge is codified, for example, in a document. Nevertheless, innovation may also relate to knowledge as an asset, if knowledge resides within individuals' expertise. This may specifically be the case in consultancies where "knowledge is the core asset" (Hansen et al., 1999, p.106), which can be commercialised in the form of consultancy services.

\section{Tacit and explicit knowledge characteristics}

Since Polanyi (1966) postulated that "we know more than we can tell" (p.4), knowledge has been differentiated into explicit and tacit knowledge. Explicit knowledge is factual knowledge that can be easily transferred, such as is found in documents. Tacit, also called implicit, knowledge is intuitive knowledge that is generally gained through personal experience (Nonaka \& Takeuchi, 1995). This distinction is significant for knowledge management, as it differentiates knowledge that is captured in individuals from explicit knowledge which is stored, for example, in documents. Tacit individual knowledge cannot be easily transferred or even stored on a physical device as is possible with explicit knowledge. Examples of tacit knowledge can be "knowledge of wine tasting, crafting a violin, or interpreting a complex seismic printout of an oil reservoir” (Nonaka \& Krogh, 2009, p.635).

Nonaka et al. (2000) suggest a model for converting knowledge. Four modes of conversion are proposed: (1) conversion from tacit into explicit knowledge, (2) converting explicit into tacit knowledge, (3) converting tacit knowledge into new tacit knowledge and (4) explicit knowledge into new explicit knowledge. They promote the possibility of transferring tacit into explicit knowledge by conducting "creative 
and essential dialogue, the use of 'abductive thinking', the use of metaphors in dialogue for concept creation, and the involvement of the industrial designers in project teams" (p.7). In particular, they believe that the successful transfer of tacit into explicit knowledge depends on the following three consecutive steps: the use of metaphor, analogy and mental model. Metaphor is a form of figurative language and "a distinctive method of expression" (Nonaka, 1991, p.100). It expresses knowledge in an intuitive way by using imagination and symbols and triggers the knowledge creating process (Nonaka, 1991). Analogy is defined as "an intermediate step between pure imagination and logical thinking" (Nonaka, 1991, p.100), thus it initially clarifies and structures the meaning of metaphors and is based on rational thinking (Nonaka, 1991). The mental model illustrates "a working model of the world by creating and manipulating these analogies" (Nonaka, 1994, p.16). It is defined as the final step in Nonaka's (1994) knowledge creation process and can be represented as systematic, consistent and transferable concepts in the form of schemata, paradigms, beliefs and viewpoints (Nonaka, 1994). These three steps - metaphor, analogy and the mental model - define the process that converts tacit into explicit knowledge as the requirement for transferring knowledge to others.

Tacit and explicit knowledge can also be understood as uncodified and codified knowledge. According to Boisot's (1987) knowledge category model, which is shown in Table 1, knowledge can be either articulated (codified) or tacit (uncodified). Boisot (1987) uses the term 'codified' to refer to knowledge that can be readily prepared for transmission purposes, for example in documents. Likewise, the term 'uncodified' refers to knowledge that cannot be easily prepared for transmission purposes, for example experiences. Boisot (1987) represents diffused knowledge as knowledge that is readily transferable to a group, an organisation or to an inter-organisational domain, while undiffused knowledge refers to knowledge that resides within individuals and is not easily accessible by others. 
Table 1: Boisot's knowledge category model (McAdam \& McCreedy, 1999, p.99)

\begin{tabular}{|c|c|c|c|c|}
\hline & Individual & Group & Organisation & $\begin{array}{c}\text { Interorganisational } \\
\text { domain }\end{array}$ \\
\hline $\begin{array}{l}\text { Articulated } \\
\text { knowledge }\end{array}$ & $\begin{array}{l}\text { Knowledge } \\
\text { calculus } \\
\text { (Type One) }\end{array}$ & $\begin{array}{l}\text { Quality circle's } \\
\text { documented } \\
\text { analysis of its } \\
\text { performace } \\
\text { (Type Three) }\end{array}$ & $\begin{array}{l}\text { Organisation } \\
\text { chart } \\
\text { (Type Three) }\end{array}$ & $\begin{array}{l}\text { Supplier's } \\
\text { patents and } \\
\text { documented } \\
\text { practices } \\
\text { (Type Three) }\end{array}$ \\
\hline $\begin{array}{l}\text { Tacit } \\
\text { knowledge }\end{array}$ & $\begin{array}{l}\text { Cross-cultural } \\
\text { negotiation skills } \\
\text { (Type Two) }\end{array}$ & $\begin{array}{l}\text { Team } \\
\text { coordination in } \\
\text { complex work } \\
\text { (Type Four) }\end{array}$ & $\begin{array}{l}\text { Corporate } \\
\text { culture } \\
\text { (Type Four) }\end{array}$ & $\begin{array}{l}\text { Customer's } \\
\text { attitudes to } \\
\text { products and } \\
\text { expectations } \\
\text { (Type Four) }\end{array}$ \\
\hline
\end{tabular}

In Table 1, Boisot's model indicates four particular stages of the described knowledge types: (1) codified and undiffused, (2) uncodified and undiffused, (3) codified and diffused, and (4) uncodified and diffused. These four combinations relate to the eight examples shown in Table 1. Types One and Two relate to individual knowledge, whereas types Three and Four cover group, organisational and inter-organisational domain knowledge. The following sections describe these types more in detail.

If knowledge is categorised as both codified (articulated knowledge) and undiffused (individual), which is represented by the upper left part of Table 1, then the knowledge is referred to as proprietary knowledge. In this case, the knowledge is ready for transfer but still resides within the individual as a knowing calculus. Also, knowledge can be uncodified and undiffused, which is related to personal knowledge, for example in the form of perceptions, experiences or cross-cultural negotiation skills. Codified and diffused knowledge is termed public knowledge and can, for example, be found in journals, books, organisational charts or supplier's patents and documented practices. Finally, common sense knowledge is relatively diffused but also uncodified. This type of knowledge is considered by Boisot (1987) to evolve slowly through a process of socialisation, harbouring customs and intuition. His model suggests corporate culture or customers' attitudes to and expectations of products for this type of knowledge. 
There are some parallels between Nonaka's (1994) thinking and that of Boisot (1987). For example, Nonaka's categorisation of explicit and tacit knowledge shows similarities to Boisot's codified and uncodified knowledge. However, both suffer from the same limitations insofar as codified and uncodified knowledge are but two discrete categories of knowledge. Moreover, the idea of diffused knowledge is rather general and it is not clear whether it comprises knowledge incorporated within the organisation together with diffusing knowledge to other organisations.

Nevertheless, tacit knowledge may not always be transferable into explicit knowledge for a number of reasons. For example, due to a lack of motivation, fear of losing knowledge and therefore competitive advantage, and cost, it might be inefficient to try converting tacit into explicit knowledge. Also, as a result of past unsuccessful attempts, technology for knowledge sharing does not seem to be an efficient means for supporting such a transfer (Connell, Klein \& Powell 2003). Another barrier to explicating knowledge may be a lack of personal awareness about having specific knowledge that could be shared with others.

Alternative knowledge dimensions

Apart from the differentiation between tacit and explicit knowledge, knowledge can be distinguished by several other means which are shown and explained in Table 2. The selected dimensions are often mentioned in knowledge management (KM) literature and they also appear to be relevant to knowledge transfer in R\&D networks. Table 2 follows the chronological order of the emergence of these dimensions. 
Table 2: Dimensions of knowledge

\begin{tabular}{|c|c|}
\hline Knowledge & Explanation and relevance of dimensions \\
\hline $\begin{array}{l}\text { Scientific knowledge and knowledge of the } \\
\text { particular circumstances of time and place } \\
\text { (Hayek, 1945) }\end{array}$ & $\begin{array}{l}\text { Scientific knowledge may be well researched } \\
\text { facts and figures, but also complex in nature. } \\
\text { R\&D networks develop and provide this highly } \\
\text { specified scientific knowledge. } \\
\text { Knowledge of the particular circumstances of } \\
\text { time and place refers to context specific } \\
\text { knowledge whereas scientific knowledge aims } \\
\text { to generalise results. }\end{array}$ \\
\hline $\begin{array}{l}\text { Knowledge as an object and knowledge as } \\
\text { a process (Hansen et al., 1999; Zack, } \\
\text { 1999) }\end{array}$ & $\begin{array}{l}\text { Knowledge can be viewed as a reusable object } \\
\text { or as a process (knowledge cycle). Creating } \\
\text { knowledge may be seen as a process, whereas } \\
\text { knowledge that is ready for commercialisation } \\
\text { represents an object. }\end{array}$ \\
\hline $\begin{array}{l}\text { Organisation-internal knowledge and } \\
\text { organisational external knowledge (Maier, } \\
\text { Haedrich \& Peinl, 2005) }\end{array}$ & $\begin{array}{l}\text { Knowledge is transferred more easily within an } \\
\text { organisation than between its boundaries, } \\
\text { however knowledge exchange between } \\
\text { organisations becomes increasingly popular. }\end{array}$ \\
\hline $\begin{array}{l}\text { Individual knowledge and organisational } \\
\text { knowledge (Maier et al., 2005) }\end{array}$ & $\begin{array}{l}\text { Individual knowledge represents knowledge } \\
\text { combined with personal experiences that } \\
\text { resides within an individual whereas } \\
\text { organisational knowledge often refers to } \\
\text { organisational memory or organisational } \\
\text { learning. }\end{array}$ \\
\hline $\begin{array}{l}\text { Electronically accessible knowledge and } \\
\text { electronically inaccessible knowledge } \\
\text { (Maier et al., 2005) }\end{array}$ & $\begin{array}{l}\text { Knowledge can be electronically accessible for } \\
\text { example in the form of documents or discussion } \\
\text { forums. }\end{array}$ \\
\hline $\begin{array}{l}\text { Formal knowledge and informal knowledge } \\
\text { (Maier et al., 2005) }\end{array}$ & $\begin{array}{l}\text { Often formal knowledge needs to follow a } \\
\text { certain formal presentation; informal knowledge } \\
\text { refers to conversation and word-of-mouth } \\
\text { knowledge and to knowledge disseminated } \\
\text { within communities. }\end{array}$ \\
\hline $\begin{array}{l}\text { Secured knowledge and unsecured } \\
\text { knowledge (Maier et al., 2005) }\end{array}$ & $\begin{array}{l}\text { Security of knowledge plays an important role, } \\
\text { especially within an inter-organisational context. } \\
\text { This may also refer to securing intellectual } \\
\text { property. }\end{array}$ \\
\hline
\end{tabular}


In addition to the described types of knowledge in Table 2, the existence of metaknowledge has a relatively long tradition in IS and knowledge management research (e.g. Carayannis, 1999; Chaudhury \& Agrawal, 2002; Cross, Borgatti \& Parker 2001). In general, meta-knowledge is knowledge (awareness) about knowledge (Carayannis, 1999; Chaudhury \& Agrawal, 2002). In the inter-organisational knowledge transfer context, this could be the knowledge about an expert's contact details or about references to other sources of knowledge (e.g. literature).

\subsubsection{Summary}

In summary, Section 2.1 introduced inter-organisational knowledge transfer, and then outlined the importance of different types of knowledge in the knowledge management context. This included a description of knowledge in the context of commercialising innovation, the distinction between tacit and explicit knowledge and a number of alternative knowledge characteristics such as formal/informal knowledge, electronically accessible or inaccessible knowledge and meta-knowledge. The types of knowledge important for the inter-organisational R\&D context of this study are various. For example, commercialising innovation in the form of explicit knowledge (knowledge as an object) or implicit knowledge (consultancy work) is important to organisations in the $R \& D$ sector. In particular, for the interorganisational context, the types of knowledge in Boisot's (1987) model seem important: attitudes (e.g. customers' attitudes) or patents and documentation. In addition, organisation internal and organisation external knowledge are significant within the inter-organisational context, as knowledge is considered a key asset in R\&D firms. For example, organisation internal knowledge may be secured in regard to external access. Moreover, in the R\&D context, scientific knowledge is a key type. With the aim of this study to suggest appropriate ICT support for inter-organisational knowledge transfer, electronically accessible and inaccessible knowledge is also considered important.

The next section provides an overview of knowledge transfer in research and development (R\&D) networks. 


\subsection{R\&D networks}

\subsubsection{Introduction}

An R\&D network consists of a range of resources, people and firms that are tied together, jointly creating and sharing knowledge between specialised fields in the network. An R\&D network is characterised by a highly dynamic interdependency between its organisations, thus it is complex in nature (Todeva, 2006). Moreover, "R\&D networks are perhaps one of the most renowned examples of knowledgesharing business relationships" (Todeva, 2006, p.189).

In Figure 1 a research network is shown to consist of a group of organisations with the research institute as the focal point. The other organisations in the network are local and national government departments, universities, industry and other related research organisations. Knowledge can be transferred in each direction between each participating organisation. This emphasises the important role of interdependence in the network.

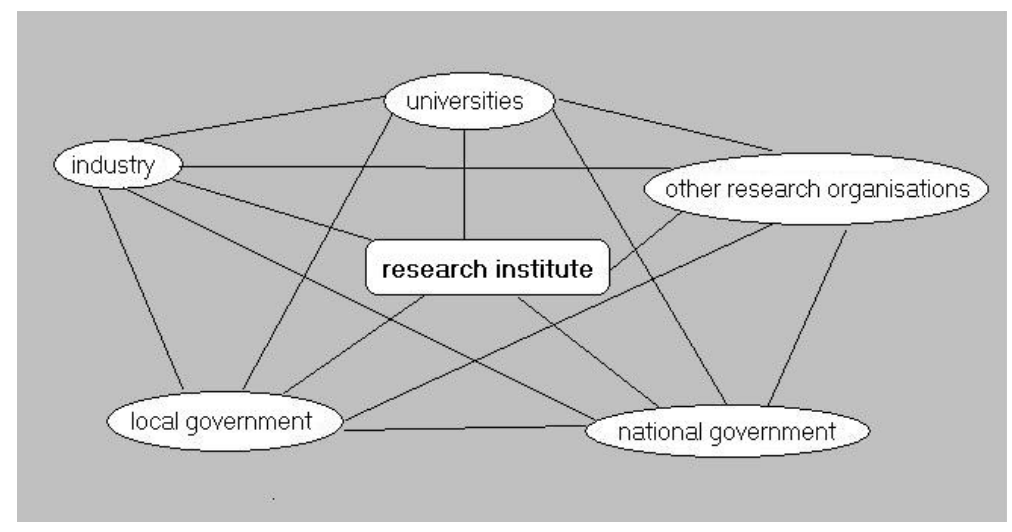

Figure 1: Potential knowledge transfer around a research institute in a business network

The remainder of this section provides a deeper insight into the R\&D context and how knowledge is shared in this inter-organisational environment. The first part identifies key themes of formal inter-organisational knowledge transfer in the context 
of R\&D networks. The second part highlights the importance of informal knowledge transfer as an essential part of R\&D networks.

\subsubsection{R\&D networks and inter-organisational knowledge transfer}

This section outlines the key literature referring to the importance of knowledge transfer in R\&D networks. Issues influencing knowledge transfer between organisations, such as management, trust and strategic goals are addressed. In this regard, strategic alliances and knowledge spillovers are introduced as key themes. In addition, commercialising as a means of transferring knowledge in the R\&D context is discussed.

\section{Strategic alliances}

Knowledge transfer in an inter-organisational environment has often been discussed in the context of strategic alliances (e.g. Badaracco, 1991; Mowery et al., 1996; Rolland \& Chauvel, 2000; Simonin, 1999). “A strategic alliance links specific facets of the businesses of two or more firms. At its core, this link is a trading partnership that enhances the effectiveness of the competitive strategies of the participating firms by providing for the mutually beneficial trade of technologies, skills, or products based upon them" (Yoshino \& Rangan, 1995, p.4). Strategic alliances are one of the

most important representations of business relationships in the R\&D sector and they can be realised in the form of joint ventures, licensing, research consortia or other joint project ties, for example with universities or other public sector institutions (Todeva, 2006). Strategic alliances can be either short-term project-based or longterm equity-based (Todeva \& Knoke, 2005). A short-term project-based alliance seems to be the most effective business relationship in today's fast-paced culture of organisational and inter-organisational changes, which is characterised by a dynamic 
interdependency between organisations (Todeva, 2006). Once these short-term project-based alliances have finished, they may either be renewed by participating organisations jointly working on another project, or former alliance partners may stay informally in contact.

Different types of strategic alliances seem to have measurable differences in terms of effective knowledge transfer. Mowery et al. (1996) confirm that a subset of alliances, which are characterised by 'convergent' development, show a significant interfirm transfer of knowledge. A 'convergent' development defines a joint development scenario in which participating organisations transfer knowledge about the same or similar development topics and goals. In contrast, an alliance consisting of a more 'divergent' development scenario is characterised by different topics and goals, and knowledge transfer does not have such an important role (Mowery et al., 1996). Nevertheless, Mowery et al. (1996) highlight a significant gap in existing research that relates to learning within alliances. They suggest further research to develop a better understanding of the learning process in this complex environment.

Factors influencing interfirm knowledge transfer such as management, trust, organisational culture, strategic goals, and the ability and willingness to communicate and learn also appear to be important in this context. For example, Cranefield and Yoong (2005) mention CEO support and expectations, competing organisational issues and traditional organisational structures as factors affecting inter-organisational knowledge transfer. Rolland \& Chauvel (2000) suggest related form, mutual trust, equal intent and similar culture as foundations for knowledge transfer in alliances, particularly learning-based alliances. Such learning-based alliances can be defined as "opportunities to learn, share, and develop new competencies in order to increase the overall portfolio of each partner" (Rolland \& Chauvel, 2000, p.227). Once these primary factors have been successfully established in an alliance, its transparency and learning capacity become relevant to achieve an effective knowledge transfer (Rolland \& Chauvel, 2000). Transparency is the willingness and ability of an 
organisation to transfer knowledge and learning capacity describes an organisation's ability to learn in an alliance.

The management of alliances is the key factor to achieving fast knowledge transfer between alliance participants (Badaracco, 1991). Badaracco's investigation into how to manage business alliances has been done in an inter-organisational and international R\&D environment. In particular, the results of Badaracco's study show that the communication in an alliance is the most important factor for achieving accelerated knowledge transfer and therefore for managing an alliance successfully.

\section{Knowledge spillovers}

Griliches (1991) defines knowledge spillover as "working on similar things and hence benefiting much from each other's research" (Karlsson, Flensburg \& Hoerte 2004, p.7). It refers particularly to knowledge transfer between basic and applied research, and between applied research and product development. However, the management of an R\&D network needs a "balance between flexible internal sharing of information, and controlled endogenous spillovers within the partnership, exogenous spillovers to the wider environment and leakage of knowledge to competitors" (Atallah, 2000 cited in Todeva, 2006, p.191). This statement reveals four different perspectives on knowledge transfer which need to be considered in R\&D alliance management:

(1) Intra-organisational knowledge transfer in each organisation participating in the alliance (flexible internal sharing of information),

(2) Controlled inter-organisational knowledge transfer (controlled endogenous spillovers within the alliance partnership),

(3) Knowledge transfer from the alliance to non-alliance organisations (exogenous spillovers to the wider environment), and

(4) Uncontrolled inter-organisational knowledge transfer in the alliance or to the outside of an alliance (leakage of knowledge to competitors). 
These four perspectives on how knowledge transfer can take place in and around an alliance indicate its potential strategic importance in terms of business success and competitive advantage. In particular, controlled endogenous spillovers, exogenous spillovers and leakage of knowledge to competitors are three significant types of knowledge transfer in the inter-organisational environment. Successfully controlled endogenous spillovers and exogenous spillovers may be part of an alliance's strategy, whereas leakage of knowledge to competitors seems to be a "form of involuntary knowledge transfer" (Lehmann \& Lehner, 2005, p.299) and is described by Zander and Kogut (1995) as imitation, which is "the capability to copy competitors' products" (Lehmann \& Lehner, 2005, p.298). A sound balance between controlled and uncontrolled knowledge transfer appears desirable in an inter-organisational environment.

As knowledge spillover can be uncontrolled and flexible in nature, one effective way of managing it may be in providing formal (controlled) R\&D services. "R\&D services are defined [...] as R\&D activities, carried out by private firms and public research organisations for external customers for payment” (Koschatzky, 2004, p.237). Research and innovation-oriented organisations and knowledge-intensive business service firms are two categories of organisations that offer R\&D services such as research work and development activities (Koschatzky, 2004). Koschatzky (2004) mentions traditional enterprise service providers, such as consultants, and firms that offer knowledge services based on new technologies, such as software development enterprises, as examples of knowledge-intensive business service firms.

\section{Commercialisation of research knowledge}

Research organisations are usually involved in commercialisation to disseminate their research knowledge for payment. According to Rogers (2003), "commercialisation is the production, manufacturing, packaging, marketing, and distribution of a product 
that embodies an innovation" (p.152). Moreover, commercialisation transforms an idea from research or practice "into a product or service for sale in the marketplace" (Rogers, 2003, p.152). Rogers (2003) defines commercialisation as the final step of three stages leading to technology transfer, which is "the application of information to use" (Rogers, 2003, p.150). The first step involves having the knowledge about the existence of an innovation. The second step comprises the application of the innovation in the organisation before finally commercialising the product or knowledge. This final step of "commercialisation requires interpersonal communication exchanges about the technology over an extended period of time" (Rogers, 2003, p.152).

In contrast to this three-step perspective, commercialisation can also be described as a process comprising a range of activities, including the focusing on and evaluating of ideas and inventions toward specific objectives, commercial development, transfer of research and/or development results, and the utilisation, dissemination and diffusion of technology-based products and knowledge (Roberts, 1988). Commercialisation can be performed "either in the form of starting up a new business, or exploiting a new business opportunity within an existing company" (Aslesen, 2005). Carr (2000), for example, investigated commercialisation of scientific research in New Zealand and considers the development of spin-offs from research organisations as one specific type of commercialisation. He points out the importance of knowledge transfer systems as a means to transfer R\&D services and therefore to commercialise new products. These services can be seminars, consultations, publications in journals, licensing of technology to commercial companies, trade expos and workshops. Thus, the provision of $\mathrm{R} \& \mathrm{D}$ services is one of the main activities that commercialises new products and knowledge.

R\&D services, specifically seminars and workshops, seem to provide a significant opportunity for participants to learn from others' experiences and even to facilitate reciprocal knowledge transfer. Thus, these services can be described as learning services which are primary represented by "tools to facilitate communication and to 
transfer implicit, personal knowledge" (Maier et al., 2005, p.286). Although seminars or workshops may initially only transfer explicit, documented knowledge, they can also include implicit knowledge transfer, for example during practical workshops. Alternatively, knowledge can be transferred implicitly between project members and/or workshop participants after attending a theoretical seminar, when basic research is applied to product development in the workplace. Thus, these learning services can enrich traditional commercialisation such as licensing or publishing with R\&D services for implicit knowledge transfer.

Moreover, learning services can consist of either traditional classroom learning or elearning. These two approaches combined provide blended learning, which "nominates an integrated, harmonised mix of methods for online, offline and face-toface learning" (Maier et al., 2005, p.292). "The term blended learning is being used with increased frequency in both academic and corporate circles" (Graham, 2006, p.3). Moreover, in corporations the term blended learning has become a buzzword during the past few years (Bonk \& Graham, 2006; Lamb, 2001). For example, the American Society for Training and Development presented blended learning in 2003 as one of the top ten trends to emerge in the knowledge delivery industry (Bonk \& Graham, 2006; Rooney, 2003). The aim is to select and blend the best possible options from traditional classroom learning and e-learning for a specific learning environment, for example for a workshop in an R\&D environment which aims to combine theoretical and practical components. This can be a significant factor in offering customised R\&D services.

In regard to the literature outlined, previous studies exploring inter-organisational knowledge transfer in networks are often concerned with driving and commercialising innovation. However, the reuse of knowledge for innovation appears significant too. This issue was, for example, investigated by Majchrzak, Cooper \& Neece (2004) who suggest a process for the reuse of knowledge. However, as knowledge often appears to be tacit, or not stored appropriately for reuse (Markus 2001; Sambamurthy \& Subramani, 2005), informal knowledge transfer may help in 
tackling this issue of reusing knowledge (Jewels, Underwood, Gregor \& de Pablos, 2004; Swap, Leonard, Shields \& Abrams, 2001). The importance of this informal transfer is described for the R\&D context in the following sections.

\subsubsection{The importance of informal knowledge transfer in R\&D networks}

This section outlines the linkage between informal knowledge transfer and workplace learning, informal key roles in the knowledge management context and informal relationships in the $\mathrm{R} \& \mathrm{D}$ sector.

Knowledge transfer and workplace learning

Workplace learning is defined as learning which takes place within a work environment. It can be formal, informal or incidental in nature (Matthews, 1999). Learning within the workplace has a number of distinctive characteristics such as being task focused, collaborative and socially embedded within the organisational context (Matthews, 1999). This makes it different from other types of learning, such as learning in schools which is cognitively different (NBEET, 1993). Further, it "often grows out of an experience or a problem for which there is no knowledge base" (Matthews, 1999, p.20).

Formal learning in the workplace might be undertaken in the form of seminars and workshops, whereas informal learning occurs through self-study, informal mentoring, asking friends and colleagues or listening to a superior (Wenger \& Ferguson, 2006). Moreover, informal social networks, such as communities of practice $(\mathrm{CoP})$, support informal learning in the workplace (Hara \& Schwen, 2006). However, learning in these groups might also occur in an incidental manner as members may participate for social reasons and not with the primary goal of transferring knowledge. 
Nevertheless, both formal and informal knowledge can be transferred between people in the workplace. Employees in a knowledge-intensive field can be categorised into knowledge seekers and knowledge providers in the most basic terms (Maier, 2001). Once identified, knowledge seekers aim to get new knowledge and thus want to learn, while knowledge providers give knowledge and thus teach the knowledge seekers directly, for example face-to-face, or indirectly, for example via documents or an elearning programme. Thus, these teaching and learning processes, or knowledge transfer processes, are closely linked to workplace learning.

In the knowledge management context, formal and informal knowledge that are transferred in the workplace are just two specific types of knowledge, as outlined in Table 2 in Section 2.1.2. Likewise, the distribution of knowledge (knowledge transfer) is just one specific task included in the management of knowledge (Maier, 2001). In fact, a variety of organisational tasks and roles is part of the management of knowledge (Maier, 2001). Other organisational tasks include identification of knowledge, storing of new knowledge, integration of knowledge in existing structure, archiving and quality assurance of knowledge (Maier, 2001). Specific roles in the KM context are responsible for these tasks. The following paragraphs describe some of these key roles.

\section{Key roles in the KM context}

Within a knowledge-intensive work environment employees often exchange knowledge in an informal manner, but their formal positions do not reflect these activities (Chan \& Liebowitz, 2006). Employees with such informal roles are often part of an informal social network. Although key roles in the KM context still seem to be informal positions in many cases, some organisations, for example professional service firms, tend to recognise the need for KM related roles. Thus, these firms establish formal positions such as knowledge manager, mentor and chief knowledge officer, who are responsible for the design, implementation and control of an organisation's KM strategy (Maier, 2001). Other important KM roles are subject matter specialist/expert (Maier, 2001; Ruggles, 1998), boundary spanner (Allen, 
1977; Cross \& Prusak, 2002; Maier, 2001; Tushman, 1977), knowledge broker (Delphi, 1997; Maier, 2001; Pawlowski \& Robey, 2004; Ruggles, 1998) and knowledge worker (Maier, 2001; Nurmi, 1998).

A subject matter specialist/expert has special expertise in one particular knowledge area and has an important role in the knowledge management context. Other possible titles for this role are subject matter expert, knowledge integrator, knowledge editor, or person responsible for a field of competence (Maier, 2001). Typically, the role of the subject matter specialist comprises the following four tasks. Firstly, a subject matter specialist, as a gatekeeper of information and knowledge, can formally approve knowledge workers' contributions to enter them into an organisation's knowledge base. Secondly, a subject matter specialist provides quality assurance in terms of reviewing and improving documents. Thirdly, he or she is the contact person for a specific topic and answers questions in his or her knowledge area. Finally, a subject matter specialist can also be a "linking pin to agencies and research institutions" (Maier, 2001, p.144).

A boundary spanner links different fields of competencies, for example while organising theme-centred workshops (Maier, 2001). In addition, the tasks of a boundary spanner can include the development of an inter-functional and interdisciplinary network of relationships. Therefore, a boundary spanner administers contacts in order to provide brokering of these contacts to other people in the network (Probst, Raub \& Romhardt, 1998). Moreover, a boundary spanner can be responsible for connecting a community to other communities. For example, one community consists of experts in the collection of water quality data, whereas two other communities are interested in the interpretation of this data. The boundary spanner would take up the role of a translator to facilitate knowledge transfer between these communities. In addition, boundary spanners can also be important knowledge brokers by transferring knowledge across boundaries (Brown \& Duguid, 1998; Davenport \& Prusak, 1998; Wenger 1998). 
The knowledge broker is considered to play a complex and multi-dimensional key role in organisational knowledge management (Cranefield \& Yoong, 2010; Delphi, 1997). A knowledge broker helps other people to find specific knowledge or to identify experts (Ruggles, 1998). The knowledge broker typically sits at the intersection between, for example, two organisations or communities (Pawlowski \& Robey, 2004). Maier (2001) suggests three levels of typical tasks for a knowledge broker: navigation, research and analysis. Firstly, a knowledge broker supports other organisational members in navigating the organisation-wide knowledge management system (KMS). A knowledge management system can be defined as "an ICT system in the sense of an application system or an ICT platform that combines and integrates functions for the contextualised handling of both explicit and tacit knowledge, throughout the organisation or that part of the organisation that is targeted by a KM initiative" (Maier, 2001, p.76). Examples for KMS could be intranet infrastructures, document and content management systems, artificial intelligence technologies, business intelligence tools, visualisation tools, groupware or e-learning systems (Maier \& Hädrich, 2006). Secondly, research-based tasks help staff to find documents and to locate experts for a given topic by accessing the KMS. Thirdly, a knowledge broker may create and analyse formal reports on a topic, an activity which encompasses summarising and connecting documents and experts in the KMS.

Furthermore, knowledge brokers work most efficiently translating topics from one community to another (Brown \& Duguid, 1998). They are able to influence the knowledge and interests of one community with the knowledge provided by another community. "In this respect, the knowledge broker also takes on the role of a boundary spanner" (Maier, 2001, p.145). Although the role of the knowledge broker is, like the boundary spanner, part of the category of knowledge intermediary roles, it is described individually in this section. A knowledge broker focuses on transferring actual knowledge rather than administering, distributing and maintaining contacts, which are the main tasks of the boundary spanner. 
"Knowledge workers are the primary target group for a KM initiative" (Maier, 2001, p.150). The ability required by a knowledge worker is defined by life-long learning and the awareness that knowledge needs to be constantly reflected, revised, extended and adapted. Quality is key to the work of a knowledge worker (Nurmi, 1998). Knowledge workers believe that the organisation supports learning. Besides working and learning with colleagues and customers, every knowledge worker is seen as an organisational profit centre (Nurmi, 1998). Knowledge workers are typically part of important formal and informal professional contacts and associations (Nurmi, 1998). With the help of a five-level model of expertise, knowledge workers can be categorised by their level of expertise (Dreyfus \& Dreyfus, 1986). The model provides the following levels of knowledge workers, beginning with those who are less experienced: novice, advanced beginner, competent worker, skilful master and expert. Knowledge workers can be knowledge providers and seekers at each of these five levels (Dreyfus \& Dreyfus, 1986). A knowledge worker who has reached the skilful master or expert level may also be a subject matter specialist.

\section{Informal relationships in the $R \& D$ sector}

Research into informal relations between scientific and technical employees in R\&D networks provides insights into the nature of their collaboration characteristics (Allen et al., 2007). Employees working in R\&D tend to build very strong trust relationships with their colleagues. Thus, they turn to these relationships for assistance and advice and do not consider alternative sources, such as other personnel or data sources (Allen, 1977).

Therefore, these informal social relationships among employees appear significant in the effort to improve and increase collaboration between R\&D firms. These informal channels of communication reflect the working relationships between individuals more accurately than formal channels (Cross et al., 2002a). 
In addition, informal communication networks are characterised by more linkages than formal ones (Kratzer, Gemünden \& Lettl, 2008). Kratzer et al. (2008) report that these informal linkages appear helpful for a team's creativity, but they do not support a team's time efficiency. With their investigation of formal and informal communication structures, they studied the use of managerial knowledge to support multi-team research and development $(\mathrm{R} \& \mathrm{D})$ projects.

Further, informal relationships are the ones which really encourage the exchange of knowledge. Informal networks are crucial to the work of scientific and technical employees, as they can cross organisational and geographical boundaries. They also create the ability for the firm to innovate (Cross \& Parker, 2004). Thus, it is very important to understand how informal networks within R\&D are formed, how they are structured and how they work (Cross et al., 2002b).

In an example of buyer-supplier relationships in small to medium-sized enterprises (Moreira, 2009), social interaction and informal knowledge transfer played a significant role in addition to formal communication structures. Specifically, in situations with problem-related information and also for the dissemination of project results, knowledge was transferred informally. A key conclusion of Moreira's (2009) study was that clients need to focus on their interaction-oriented capabilities.

\subsubsection{Summary}

This section summarised the key contextual factors underpinning this study. First, the importance and challenges of knowledge transfer in R\&D networks, especially in alliances, was pointed out by addressing influencing factors and the management of $R \& D$ alliances. Knowledge spillover was identified as the term used to describe knowledge transfer in an inter-organisational R\&D context. $R \& D$ organisations are interested in commercialising their scientific knowledge. R\&D services, especially learning services, can make a significant contribution to commercialisation. 
This review of the contextual literature also found that informal knowledge transfer is an essential part of larger R\&D networks. Such informal collaboration is based around specific research topics and allows participants to learn in an informal way. Thus, learning also plays an important role in R\&D networks. In addition, this section described several informal key roles for knowledge transfer such as knowledge broker, subject matter specialist and knowledge worker. Moreover, there appears to be a lack of research investigating informal collaboration and knowledge transfer in R\&D networks, particularly in the social network context. Previous research has explored the role of knowledge transfer in social networks which the next section expands upon. 


\subsection{Knowledge transfer in social networks}

The following sections define and describe knowledge transfer from a social network perspective.

\subsubsection{Social networks}

A social network is a set of social entities, such as individuals, groups or organisations, which are connected to each other in order to exchange information or other resources (Haythornthwaite, 1999). A social network perspective focuses on relationships between these entities, which can be represented, for example, by "communications among members of a group, economic transactions between corporations, and trade or treaties among nations" (Wasserman \& Faust, 1994, p.i). It also highlights the importance of "informal, interpersonal relations in social systems" (Scott, 2000, p.7).

\subsubsection{Knowledge transfer from a social network perspective}

A social network perspective applied to the context of knowledge management helps identify and analyse knowledge transfer in organisations (Anklam, 2002). The goal is to achieve a better understanding of existing knowledge transfer structures and processes and to therefore analyse their patterns. One support mechanism could be interventions for creating, reinforcing, or changing knowledge transfer patterns (Anklam, 2002).

The social network perspective appears most significant in regard to informal networks (Liebowitz, 2005) as in comparison to informal networks, the formal structures underpinning organisational charts do not usually reflect the actual knowledge transfer structures (Chan \& Liebowitz, 2006). The application of the social network perspective to knowledge management can be seen as a means "to push the new frontiers of knowledge management" (Chan \& Liebowitz, 2006, p.19). 
There are several factors, such as the frequency, direction and type of knowledge and structural network properties that characterise the nature of knowledge transfer. In particular, the types of relationship between participants in a network may influence their knowledge transfer. Hansen (1999) investigated the impact of tie strength and type of knowledge on knowledge transfer. Tie strength is a typical term used in a social network context and it represents the strength of a relationship, which can be measured by the frequency of contacts. A high frequency of contacts reveals a strong tie, whereas less frequent contacts represent a weak tie. Table 3 shows Hansen's (1999) findings.

Table 3: Transfer effects associated with four combinations of knowledge complexity and tie strength (adapted from Hansen, 1999 p. 89).

\begin{tabular}{|l|l|l|}
\hline \multirow{2}{*}{\begin{tabular}{l} 
KNOWLEDGE \\
Non-codified, \\
\cline { 2 - 3 } Dependent
\end{tabular}} & $\begin{array}{l}\text { TIE STRENGTH } \\
\text { problems }\end{array}$ & Weak \\
\hline $\begin{array}{l}\text { Codified, } \\
\text { Independent }\end{array}$ & $\begin{array}{l}\text { Few transfer } \\
\text { problems } \\
\text { problems }\end{array}$ \\
& & $\begin{array}{l}\text { Few transfer } \\
\text { problems }\end{array}$ \\
\hline
\end{tabular}

The four results presented in Table 3 combine the type of knowledge and tie strength. The type of knowledge is expressed by either non-codified and dependent knowledge or codified and independent knowledge. Codified and non-codified knowledge refer to explicit and tacit knowledge. Dependent knowledge represents knowledge that is part of a larger system and is therefore not as easy to transfer as independent knowledge (Hansen, 1999). Thus, the non-codified, dependent knowledge type appears to be more complex in nature. However, both types of knowledge have been categorised by tie strength (strong, weak) in order to present results on the efficiency of knowledge transfer. 
However, the type of relationship was not the main influence on the efficiency of knowledge transfer in Hansen's study (1999). Neither mainly weak nor mainly strong relationships between members of different organisational sub-units could be determined in order to achieve efficient knowledge transfer (Hansen, 1999).

Moreover, the type of knowledge, specifically the complexity of knowledge, has the strongest impact on efficient knowledge transfer according to the completion time of a project (Hansen, 1999). In this case, strong relationships transfer this complex knowledge better than weak relationships. Conversely, weak relationships transfer non-complex knowledge better and they are even more cost-efficient as there is no need to maintain redundant contacts, which are associated with maintaining strong relationships (Hansen, 1999).

Hansen (1999) categorises the results into few, moderate or severe knowledge transfer problems. Codified and independent knowledge can be transferred easily between strong and weak ties. However, the more complex knowledge causes moderate transfer problems for strong relationships and severe transfer problems for weak ties.

Although the types of knowledge transferred and the notion of tie strength appear to be important for knowledge transfer in social networks, Reagans and McEvily's (2003) findings indicate that other factors such as social cohesion and network range affect knowledge transfer more than tie strength between two people.

Social cohesion and network range are distinct features of a network's structure Social cohesion describes the embeddedness in a dense network structure of thirdparty relationships (Granovetter, 1973; Hansen, 1999). Network range describes relationships that span multiple knowledge sources. Social cohesion and network range are likely to have distinct but complementary effects on knowledge transfer (Reagans \& McEvily, 2003). Whereas social cohesion highlights the value of thirdparty relationships, network range points to the benefits associated with network 
connections that span important organisational boundaries (Reagans \& McEvily, 2003).

According to Reagans and McEvily (2003), social cohesion and network range facilitate knowledge transfer regardless of the associated tie strength between two people. Nevertheless, strong ties facilitated the transfer of tacit knowledge more than they facilitated the transfer of codified knowledge, which confirms Hansen's (1999) results.

Besides these structural properties of networks, the ontological network structure may also play an important role in inter-organisational knowledge transfer.

\subsubsection{Ontological network structures and knowledge transfer}

Social networks in the context of knowledge transfer can be viewed as Information Processing Networks (IPNs). These networks may have various structures that influence knowledge transfer. Such ontological network structures can be categorised as decentralised or centralised structures (Kwon et al., 2007).

In terms of various ontological structures that have been suggested for Information Processing Networks (IPNs) (Kwon et al., 2007) and based on the literature on network theory (e.g. Albert \& Barabasi, 2000), a decentralised network may either have random network structure or a small-world network structure. The random network structure shows the highest level of decentralisation.

In random network models all node connections are evenly distributed (Poisson distribution) and no single node controls all the other connections. Therefore all nodes are roughly equally important in a given network. Based on graph theory, this model was introduced by Erdos and Renyi (1960) nearly five decades ago. Random 
network structures may appear in an organisation in which employees "communicate laterally, process a fairly similar amount of information, and have an equal share of responsibility" (Kwon et al., 2007, p.210). This can particularly apply to technical work and other types that include non-routine tasks (Ahuja \& Carley, 1999; Hinds \& Kiesler, 1995). According to Kwon et al. (2007), the structural design of the random network may be most appropriate for knowledge-intensive industries including software engineering, biotechnology, $R \& D$ and consulting. Lateral communication and information processing for collaborative tasks are significant in these knowledgeintensive industries (Kwon et al., 2007). Figure 2 illustrates a random network structure.

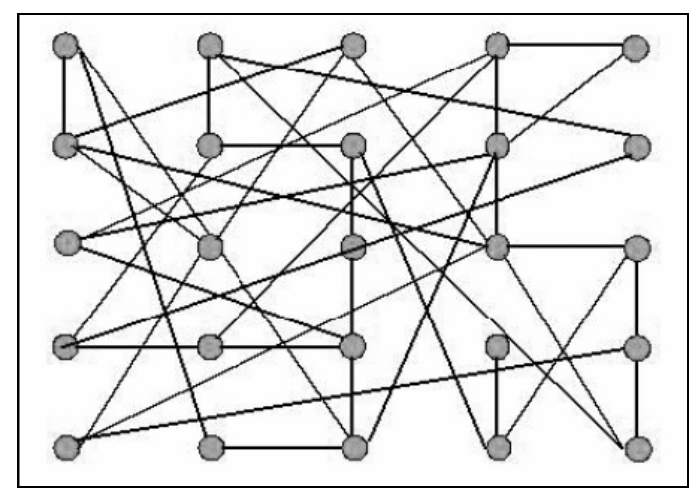

Figure 2: Random network structure

In contrast to a random network structure, a small-world network structure includes some degree of centralisation. Although small-world networks also consist of randomly structured relations, they include some local clusters that represent some level of centralisation (Kwon et al., 2007). Thus, the small-world networks can be differentiated from pure random networks by their degree of randomness (Watts \& Strogatz, 1998).

Small-world networks that are typical in many large-scale real-world networks such as biological networks, the Internet or social networks (Nguyen, 2006) have a high number of direct connections between any two network members (Albert \& Barabasi, 2000). Another example identified a large social network of people using the same instant messaging tool (Leskovec \& Horvitz, 2008). 
Local clustering facilitates reliable access, whereas links between local clusters in a small-world network can provide easy access in the network (White \& Houseman, 2003). In a small-world network ICT can efficiently support information processing (Kwon et al., 2007). ICT allows for connections across different groups, and these connections help encourage mutual adaptation and collaboration among members. The following figure shows a small-world network structure.

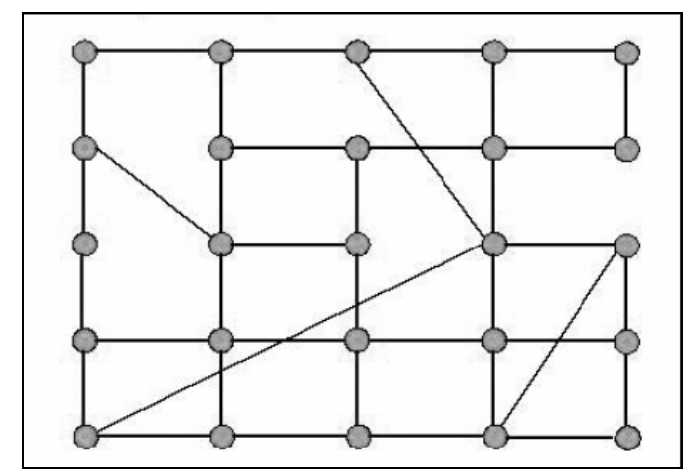

Figure 3: Small-world network structure

Small-world networks have been recommended by several authors as the ideal interorganisational structure for networks such as alliances to facilitate knowledge transfer and creation (e.g. Cowan, 2004; Cowan \& Jonard, 2004) and optimise their output (Schilling \& Phelps, 2007).

In contrast to these decentralised structures, centralised structures show a much higher degree of centrality represented by a single central actor (e.g. organisation or department) within a network. Centralised structures can be differentiated into Barabasi and moderate scale free networks (Kwon et al., 2007). Barabasi structured networks show a higher centralisation than moderate scale free networks. Younger organisations such as entrepreneurial organisations with a simple and very centralised structure are examples of Barabasi structured networks (Kwon et al., 2007); law firms and universities are examples of moderate scale free structured networks, as they show an overall fewer degree of centralisation but indicate the existence of local powerful hubs (e.g. certain departments within an organisation). 
Besides these structures, key knowledge transfer processes such as advice giving and seeking activities play another important role in inter-organisational knowledge transfer.

\subsubsection{Advice giving and seeking as key knowledge transfer processes in social networks}

A considerable number of previous studies used advice giving and seeking activities as a type of knowledge transfer when looking at how people transfer knowledge in their professions (e.g. Cross et al., 2001; Haythornthwaite, 1996; Krackhardt \& Hanson, 1993). These advice giving and seeking activities in the workplace appear to reflect informal knowledge transfer processes. Work colleagues could seek advice such as another colleagues' previous work experiences, process knowledge or simply information about contact details or references to other sources of knowledge (e.g publications).

Cross et al. (2001) investigated the dimensions of the advice network to explore what really is transferred when organisational members go to each other for work-related advice. It has often been assumed that more than simple answers pass between these people (Allen, 1977; Hansen, 1999; Rogers, 2003; Szulanski, 1996; Weick, 1995). In this regard, Cross et al. (2001) identified several dimensions of the advice network: solution, meta-knowledge, problem reformulation, validation and legitimation. Their results also show that organisations looking at knowledge sharing and learning typically recognise only who goes to whom for a solution, while the even more important dimensions of meta-knowledge, problem reformulation, validation, and legitimation are neglected (Cross et al., 2001). In particular, the dimensions of metaknowledge and problem reformulation appear to be informal in nature.

In the R\&D context in particular, advice networks play a significant role in informal knowledge transfer. Employees working in $R \& D$ tend to build very strong trust 
relationships with their colleagues. Thus, they turn to these relationships for assistance and advice and do not consider alternative sources, such as other personnel or data sources (Allen, 1977).

Moreover, in the social network literature, advice giving and seeking activities are also mentioned as a key indicator for investigating relationship strength among actors in a network (e.g. Mathews, White, Soper \& Bergen, 1998; Petróczi, Nepusz \& Bazsó, 2007).

In addition, the level of interaction in advice networks is an important aspect to be considered. Varying high levels of interaction may also reflect knowledge transfer activity. In this regard, the direction of knowledge transfer appears important, as it indicates knowledge givers and seekers. These high levels of knowledge giving and seeking activities therefore indicate authoritative positions in an advice network (Brogan \& Armstrong, 2005).

\subsubsection{Summary}

This section described key literature in the field of knowledge transfer in social networks. First, social networks were defined briefly. Then, the ontological structures of networks and the key processes of advice giving and seeking activities were outlined. Literature that investigated knowledge transfer in relation to social network properties such as tie strength and network structure was described. Overall, the social network perspective has been previously applied to the knowledge management context and appears highly relevant for further investigations into knowledge transfer in social networks. The following section outlines possible ICT to support knowledge transfer in an inter-organisational context. 


\subsection{ICT support for knowledge transfer}

\subsubsection{Introduction}

Information and communication technology (ICT) for knowledge transfer is ideally part of an organisation-wide KM strategy (Hansen et al., 1999). The main goal of a $\mathrm{KM}$ strategy is to align an organisation's knowledge resources and knowledge-related capabilities to the knowledge requirements of its business strategy (Maier, 2001; Zack, 1999). Moreover, an organisation's KM strategy determines whether it views knowledge as largely stored in computers, or as residing in people.

Organisations which rely on their tacit knowledge, for example scientific organisations that create highly complex knowledge, may follow a strategy that supports knowledge transfer between people. This strategy may also affect the choice of technologies to support this knowledge transfer. However, in terms of an interorganisational environment, the individual $\mathrm{KM}$ strategies of organisations participating in a network need to converge and together represent the network's KM goals.

For knowledge transfer, tools and systems specifically developed for supporting knowledge management appear to be the most appropriate choice as part of a larger ICT pool. Apart from the term knowledge management systems (KMS), there are several other terms in use, among them knowledge infrastructure, knowledge warehouse or knowledge-oriented software (Maier et al., 2005). These terms are used to describe functionalities supporting knowledge workers, for example, in the creation, identification, acquisition, selection, linking, structuring, application and maintenance of knowledge (Maier, 2001).

Besides KMS, there is also another group of software systems that supports knowledge management initiatives: e-learning software, such as e-learning suites, learning management platforms or portals (Maier et al., 2005). All these systems 
appear to include typical components for document management, learning management and collaboration.

"The focus on information and communication technologies (ICT) as a medium for collaboration has gradually increased over the last two decades" (Munkvold, 2003, p.1). Beginning with the development of groupware in the 1980s, collaborative features are now embedded in many standards tools in the workplace. Collaboration has also become an integral part of many current strategic initiatives, such as KM, virtual teams or global collaborations. Nonetheless, applying collaborative technologies in order to increase and improve collaboration remains a challenge for many organisations (Munkvold, 2003).

Moreover, in an inter-organisational collaboration context, additional critical factors such as "complex regulatory issues involved in merging various networks and utilities into the larger communications infrastructure are becoming apparent" (Branscomb, 1992 cited in Pickering \& King, 1995, p.479).

The remainder of this section firstly introduces three models that provide insights into ICT-supported knowledge transfer in the KM context. Then, ICT support for knowledge transfer from a social network perspective is described. This approach to presenting diverse perspectives on ICT support for knowledge transfer hopes to give a comprehensive overview of opportunities.

\subsubsection{ICT support for knowledge transfer from a KM perspective}

In this section, three alternative models suggesting ICT support for knowledge transfer are outlined: Kwan and Cheung's (2006) model of the knowledge transfer process, Despres and Chauvel's (2000) knowledge transfer mechanisms and Wenger and Ferguson's (2006) knowledge ecology model. The tools and technologies mostly 
addressed by these three models refer to knowledge transfer among individuals and within groups. However, Kwan and Cheung's (2006) model also provides some insights into ICT support at the inter-organisational level. These three models show specific characteristics deemed useful for this study, which aims to suggest ICT support for knowledge transfer and learning in inter-organisational social networks. These characteristics include an inter-organisational perspective (a model of the knowledge transfer process), a focus on dissemination breadth and knowledge characteristics (knowledge transfer mechanisms) and a focus on ICT support for learning (knowledge ecology model).

\subsubsection{Model of the knowledge transfer process}

Firstly, the model of the knowledge transfer process (Kwan \& Cheung, 2006) provides a more detailed description of several stages of the knowledge transfer process. This model presents knowledge transfer as a four step process which is embedded within an organisation. Kwan and Cheung's (2006) model is mainly based on Hansen's (1999) two stage knowledge search and transfer model and also on Szulanski's (1996, 2000) four stage knowledge transfer model which considered initiation, implementation, ramp-up and integration as the main phases.

Kwan and Cheung (2006) present four main stages in their knowledge transfer process: (1) motivation, (2) matching, (3) implementation, and (4) retention. The four stages are linked by certain conditions. Each stage, except for the first one, has such a predefined assumption, which consists of the earlier stage's result. For example, the condition for the matching stage is that there has already been an actual attempt to search for a knowledge transfer partner (motivation).

The motivation stage and the matching stage are iterative phases. The process starts with the identification of existing knowledge gaps and the target knowledge which is needed to fill this gap. However, the discovery of such a gap does not automatically 
initiate a search for potential solutions as inhibitors, such as a lack of understanding and motivation or organisational culture, may hinder this part of the process. The source or the recipient of knowledge needs to start to communicate their intention to transfer knowledge. After this initial search for a corresponding partner, the attempt to transfer is developed in the matching stage. However, this part of the process may take a while and there is an iterative loop between the two stages before both partners are motivated and the transfer is ready to proceed. Then, in the implementation stage knowledge actually flows between recipient and source. Also, new knowledge is initially used by the recipient between the implementation and the retention phase, which maintains the knowledge transfer relationship.

Kwan and Cheung (2006) suggest specific KM tools such as brainstorming tools, knowledge maps, knowledge repositories, and groupware or CoP tools as part of an overall knowledge transfer management system (KTMS) in order to support the knowledge transfer process. It is defined as an integrated system of current KM tools designed to support different needs throughout the knowledge transfer process. Brainstorming tools and knowledge repositories may help to identify gaps between existing and target knowledge. Likewise, knowledge maps support the understanding of relationships in order to bridge this gap. Groupware, CoP tools and knowledge repositories are proposed "to support resource and communication flow between the transfer partners" (Kwan \& Cheung, 2006, p.28).

\subsubsection{Knowledge transfer mechanisms}

The model of Knowledge transfer mechanisms (Despres \& Chauvel, 2000) illustrates a range of different ICT and non-ICT mechanisms, which are mapped to support a specific dissemination breadth with either tacit (low ability to codify) or explicit (high ability to codify) knowledge. Figure 4 illustrates this model. 


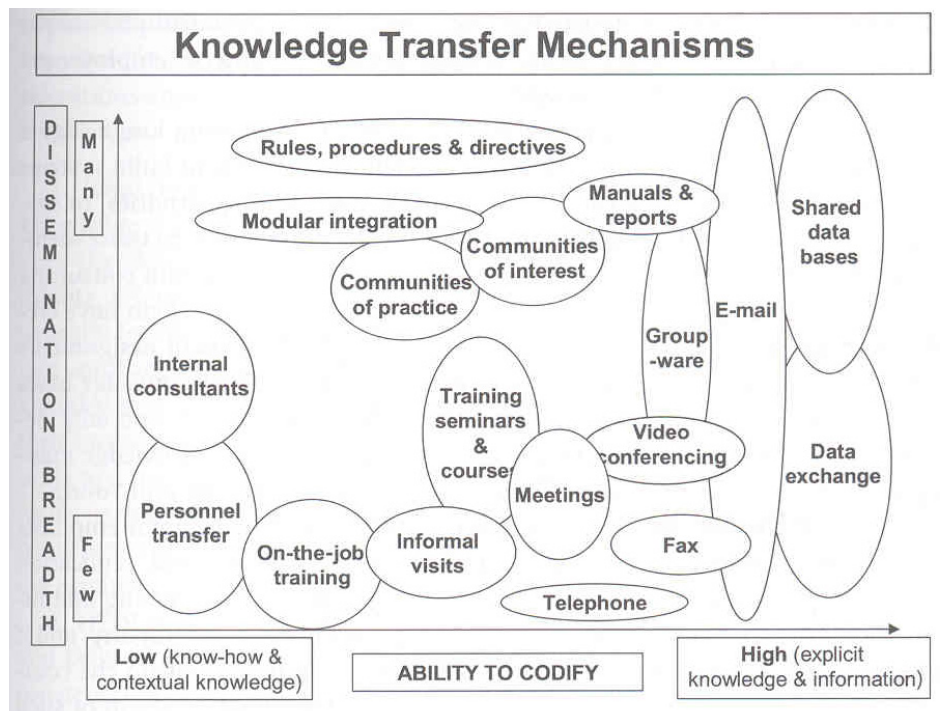

Figure 4: Knowledge transfer mechanisms (Despres \& Chauvel, 2000, p.49)

Figure 4 shows several mechanisms sorted by two dimensions relating to knowledge transfer. The ability to codify knowledge is considered on the horizontal axis, whereas the dissemination breadth, which shows how many or how few people can be reached by different means of knowledge transfer, is considered on the vertical axis. Overall, eighteen different methods and tools ranging from personnel transfer and training/seminars to email and data exchange are illustrated. The ability to codify is differentiated by a low ability, which refers to codifying of know-how and contextual knowledge, and by a high ability, which takes explicit knowledge and information into account.

In Despres and Chauvel's (2000) model, ICT examples to support knowledge transfer are shared databases, email, groupware and videoconferencing. Whereas shared databases and email may also be part of groupware, videoconferencing appears to provide another suitable alternative. Videoconferencing can consist of both point-topoint conferences and multi-point conferences (Maier, 2001). Whereas point-to-point videoconferences connect two people by voice and video, multi-point videoconferences are able to link more than two participants and thus require a special technological infrastructure (Maier, 2001). 
Furthermore, some other transfer mechanisms, such as informal visits, training seminars and courses and on-the-job training, which are not defined as explicit ICT tools in the model, could also be replaced or enhanced by appropriate ICT. Nevertheless, apart from the dissemination breadth, this model does not consider any other form of knowledge transfer such as the frequency of transfer or direction of knowledge transfer.

\subsubsection{The knowledge ecology model}

The knowledge ecology model (Wenger \& Ferguson, 2006) is a framework that has been developed at Sun Microsystems in order to merge their changing education and training business with their knowledge management initiatives (Wenger \& Ferguson, 2006). It represents a range of "knowledge or learning options that support knowledge services" (Wenger \& Ferguson, 2006, p.87) such as collaboration, online $\mathrm{CoP}$ and learning portals. It allows the combination of "current possibilities as well as new possibilities emerging from new technologies or learning designs" (Wenger \& Ferguson, 2006, p.78). Further, it provides insights into the possible use of ICT to support knowledge transfer through these knowledge services. It supports a blended learning environment.

Four distinct groups of knowledge services are identified in the framework: (1) exploring, (2) participating, (3) informing, and (4) guiding. People explore resources for ideas and practical insights, and they participate, for example, in a community to create understanding (Wenger \& Ferguson, 2006). Further, experts inform people in common theories and practice and they also guide people as they apply their knowledge and skills to a new situation (Wenger \& Ferguson, 2006). ICT examples which belong to the exploring category include e-books and learning portals. Online CoP and instant messaging aim to support the participation group. ICT in the knowledge service category of informing can consist of online learning and virtual 
classrooms. E-mentoring, remote coaching and web conferencing are examples of guiding knowledge services.

Table 4 summarises and illustrates the ICT support for knowledge transfer indicated in the three models. All three models consider ICT support such as groupware and other collaboration tools and technologies that mainly relate to support at the individual and group level. These collaborative tools and technologies appear to support advice giving and seeking activities in the workplace. For example, learning portals or online CoPs could be a platform for inter-organisational advice giving and seeking activities. Instant messaging, for example, could support a relatively quick transfer of advice to knowledge seekers. In addition, to the individual and group level support, Kwan and Cheung's (2006) knowledge transfer process model also considers ICT support at the inter-organisational level in the form of a KTMS that would provide the technical platform to run inter-organisational collaborative tools.

Table 4: Knowledge transfer and ICT support

\begin{tabular}{|ll|l|l|}
\hline KM/KT Model & & ICT support \\
\hline $\begin{array}{l}\text { Knowledge ecology model (Wenger \& } \\
\text { Ferguson, 2006) }\end{array}$ & $\begin{array}{l}\text { E-books, learning portals, online CoP, instant } \\
\text { messaging, online learning, virtual } \\
\text { classrooms, e-mentoring, remote coaching, } \\
\text { web conferencing }\end{array}$ \\
\hline $\begin{array}{l}\text { Knowledge transfer mechanisms (Despres \& } \\
\text { Chauvel, 2000) }\end{array}$ & $\begin{array}{l}\text { Shared databases, email, groupware, } \\
\text { videoconferencing }\end{array}$ \\
\hline $\begin{array}{l}\text { Knowledge transfer process (Kwan \& } \\
\text { Cheung, 2006) }\end{array}$ & $\begin{array}{l}\text { KTMS embodying KM tools (including } \\
\text { groupware, community of practice tools, } \\
\text { brainstorming tools, knowledge maps, } \\
\text { knowledge repositories) }\end{array}$ \\
\hline
\end{tabular}

The following section outlines other possibilities for ICT to support knowledge transfer, particularly in social networks. 


\subsubsection{ICT support for knowledge transfer in social networks}

The social network perspective was identified by the researcher as an appropriate and well-established approach for exploring interaction structures among participants in a network. Moreover, the social network approach has been highlighted by a range of studies as a successful way of analysing online social networks (e.g. Garton, Haythornthwaite \& Wellman, 1997; Haythornthwaite, 1996; Willging, 2005). The following sections firstly introduce a theoretical social network concept based on media use and tie strength. This media use reflects ICT support for knowledge transfer at the individual and group level. Secondly, the following sections review studies that explore specific ICT options in order to support communication in social networks without the consideration of theoretical social network concepts. These ICT options refer to individual and group as well as inter-organisational levels.

\subsubsection{Media use and tie strength}

Weak and strong ties in a social network use a different number of media such as email, videoconferencing and face-to-face meetings to communicate with each other (Haythornthwaite, 2005). This phenomenon has been named media multiplexity. It shows a link between the number of different media that have been used by network actors, and the types of relationship (strong and weak ties) they have with other actors. However, media multiplexity does not additionally differentiate by the type of media that might be most appropriate to support specific ties. It argues that strongly tied pairs (for example friends) are more likely to use multiple media than weakly tied pairs in a network. Moreover, there does not appear to be a difference in users' ICT preferences for transferring different types of knowledge (referring to strong ties).

Haythornthwaite (2005) has also explored the impact of the implementation of new collaboration tools, such as internet relay chat, web boards, email listservs, online 
social support groups and community tools, on social networks. The resulting conceptual model has been named latent tie theory. Latent ties offer the technical possibilities for, but do not anticipate, the building of social relationships. Latent tie theory argues that the implementation of a new collaboration tool affects three significant components. Firstly, the implementation creates new latent ties and supports the formation of new weak ties. Secondly, it is also able to recast or disrupt existing weak ties. Finally, the implementation of a new collaborative tool has minimal influence on existing strong ties in a network.

In summary, latent tie theory provides opportunities for the creation of new ties, but it also involves risks for existing ties, specifically weak ties. Therefore, weak ties that are important in a network need to be taken into account when implementing a new online collaboration tool. For example, there might be a significant weak tie between two people who work in two firms that are strategically bound. A new online collaboration tool that has been implemented in just one of these firms becomes the typical collaboration tool for one of these people, but not for the other one. These different tools may contribute even more to their infrequent communication.

\subsubsection{Other ICT support for knowledge transfer in social networks}

This section firstly introduces ICT support at the individual and group level. Then, it outlines options for the inter-organisational level. Whereas the section on individual and group level support outlines tools and technology options, the section on the inter-organisational level focuses on network infrastructure and systems to support knowledge transfer and learning in social networks. 
Individual and group level support

Social software

Social software is the type of ICT used for transferring informal and tacit knowledge (Avram, 2006). Social software can be defined as "networked tools that support and encourage individuals to learn together while retaining individual control over their time, space, presence, activity, identity and relationship" (Anderson, 2005, p.4). Since the beginning of the development of internet technology, social software has been made available especially for knowledge transfer, exchange and collaboration between distributed teams (Weiss, 2005).

Thus, social technologies appear to be similar to collaboration technologies. Dalsgaard (2006) suggests a range of social technologies such as weblogs and wikis (both are online diaries), RSS feeds and social bookmarking. Whereas the first three technologies include collaborative features, social bookmarking is a webpage representing only a collection of personal bookmarks (links to other web pages), which are accessible by other people and do not offer collaborative features.

A social software perspective provides better insight into the use of collaborative elearning tools in social networks (Dalsgaard, 2006). Social networks and their interactions are considered to be a significant factor when designing, developing and implementing an e-learning platform (Cho, 2002). For example, Cho's study investigated two long-standing e-learning communities and explored how people develop social structures using online communication and collaboration tools. The study's main conclusion is that social networks, such as pre-existing friendship networks, influence the emergence of collaborative learning and working networks (Cho, 2002), and thus are important factors for consideration when planning the implementation of an organisational e-learning system. 


\section{ICT support based on synchronous and asynchronous technologies}

Another example of ICT support for knowledge transfer in social networks is the use of instant messaging tools (Cho, Trier \& Kim, 2005). Instant messaging belongs to the group of synchronous technology which provides the ability to give immediate feedback and incorporates tools such as videoconferencing and groupware (Riopelle et al., 2003). Instant messaging can be used for different communication and knowledge transfer purposes, such as discussion, socialisation or document transfer (Cho et al., 2005; Wenger \& Ferguson, 2006). Instant messaging can also help employees to improve their working relationships in order to strengthen or maintain existing relationships, or build new ones (Cho et al., 2005). However, an issue that has been raised in Cho et al.'s (2005) study is the informality of the instant messaging medium in the workplace.

Online social networks can be analysed using asynchronous online interactions (Willging, 2005). Asynchronous interactions, unlike synchronous interactions, are defined by communication patterns that do not require immediate feedback. Asynchronous technology is provided by tools such as email, voicemail, discussion forums and shared drives (Riopelle et al., 2003). The identification and description of group dynamics and interaction patterns in an online class are the focus of Willging's study (2005). Group dynamics may represent changing interaction patterns in a group. He argues that asynchronous online interactions should be, at least to some extent, part of an e-learning system for distance education. Moreover, asynchronous technology may be more useful in trying to explicate tacit knowledge. For example, when participating in discussion forums, tacit knowledge residing in individuals is converted into explicit knowledge, which is saved as a forum entry.

\section{Other collaboration technologies}

Two other categories of collaboration technologies besides synchronous and asynchronous technology deemed important to this study are firstly push and pull technology, and secondly peer-to-peer technology. Firstly, push and pull technology supports different ways of directional knowledge transfer. Push technology 
distributes knowledge automatically, once a person has subscribed to receive it (Kapyla, Niemi \& Lethola, 1998). This can be in the form of subscribing to a newsletter that will then be sent automatically to the knowledge seeker. Pull technology provides the knowledge seeker with, for example, search engine tools (Kendall \& Kendall, 1999), but knowledge seekers still need to search on their own.

In addition, peer-to-peer technology provides two or more peers in a network of equals with an ideal infrastructure for spontaneous communication (Schoder \& Fischbach, 2003). Peer-to-peer technology does not have central coordination as is the case in a client server infrastructure. However, such a central coordination component may represent a controlling unit for network participants.

Inter-organisational level

In order to use social software and other tools and technologies successfully for knowledge transfer in inter-organisational R\&D networks, organisations may need "Inter-organisational Computer Mediated Communication (ICMC) technologies" (Pickering \& King, 1995). These technologies can be defined as "the broad 'Internet' family of networks" (Pickering \& King, 1995, p.479), which may include utilities such as the World Wide Web (WWW), and interactive multimedia (Pickering \& King, 1995). Other examples for ICMC technologies are inter-organisational electronic mail and Usenet, which is a "conglomeration of newsgroups or electronic bulletin boards accessible from the Internet community" (Pickering \& King, 1995, p.481).

The merging of network technologies from different organisations into the larger ICMC infrastructure involves complex regulatory issues (Pickering \& King, 1995). Nevertheless, these ICMC infrastructures, once appropriately set up and providing necessary bandwidth and robust access to users, may contribute to a network's 
strategic KM goals, by assuring well-managed and technology-supported knowledge transfer among organisations.

However, the difficulties in merging diverse network technologies could be avoided for an inter-organisational infrastructure, for example, by establishing an Extranet VPN. This solution would allow other organisations to have secure and controlled access to parts of one organisation's intranet (Lipp, 2006). For example, an ExtranetVPN infrastructure may provide access to external users to use one organisation's intranet for the collaborative use of a portal solution for knowledge transfer (Villian, Leiner, Gaffal \& Pisl, 2004; Zheng, Chen \& Wu, 2004).

According to Pickering and King (1995), ICMC infrastructures are especially favoured by research-oriented organisations that are highly interested in maintaining external weak social ties among employees. In this context, ICMC technologies "provide the means for notification and negotiation among organisations shopping for professional services" (Pickering \& King, 1995, p.484).

The need for further research into ICT to support knowledge transfer has been recognised. Among the most identified gaps in the literature is the design and development of a KTMS to support the knowledge transfer process. Kwan and Cheung (2006) also point out the need to consider technical interfaces to other knowledge management systems (KMS) when transferring knowledge between organisations. $\mathrm{KMS}^{1}$ can be present in the form of document or content management systems, intranet infrastructures or portals (Maier \& Hädrich, 2006).

KMS can be differentiated by central and decentralised solutions. Centralised systems have advantages in co-ordinating and controlling knowledge transfer, whereas decentralised (peer-to-peer) solutions offer better options to maintain a decentralised knowledge repository (Maier \& Hädrich, 2006; Schmitz, Hotho, Jaeschke \& Stumme, 2006). Portal solutions in particular allow for sharing knowledge among a group of

\footnotetext{
${ }^{1}$ for a definition see page 33
} 
diverse users and organisations (van Baalen, Bloemhof-Ruwaard \& van Heck, 2005; Zanner, 2006). This could include yellow pages which are often part of organisational KM initiatives (Stankosky, 2005).

\subsubsection{Summary}

This chapter presented ICT support for knowledge transfer at the individual, group and inter-organisational levels. Firstly, three knowledge transfer models were described and ICT support for knowledge transfer in social networks was outlined. A summary of the three models revealed that Wenger and Ferguson's (2006) knowledge ecology model and Kwan and Cheung's (2006) knowledge transfer process model both consider ICT support at the individual, group and inter-organisational level. For example, learning portals or KTMS address the inter-organisational context, whereas other ICT support such as groupware and other collaboration tools and technologies mainly relate to ICT support at the individual or group level.

In addition, collaborative technologies, also called social software, together with ICMC infrastructures, were identified as the types of ICT that are able to support inter-organisational collaboration among social ties (individual and group level). In particular, ICMC infrastructures provide the maintenance of inter-organisational

weak social ties, which may be of strategic importance to an organisation and/or the whole network. Therefore, social networks, such as a network of research-oriented organisations, strongly support ICMC infrastructures, which help to merge their individual organisational network technologies including collaborative utilities (Pickering \& King, 1995).

Although there is a range of literature exploring knowledge transfer with social software, little research has been done to investigate ICT for knowledge transfer using a social network approach. In particular, there is a lack of research exploring knowledge transfer in e-learning environments from a social network perspective. The key theme in Dalsgaard's (2006) study, for example, is to encourage further 
research into the application of social software to learning software in order to support collaborative e-learning and therefore improve traditional learning management systems (LMS).

Although the diverse perspectives on ICT support for knowledge transfer given in this section provide a comprehensive overview of opportunities, this section is not all-encompassing. Moreover, due to presenting diverse perspectives and literature, it is often difficult to clearly differentiate among tools, technologies, systems and services. The rapidly changing character of the ICT domain and its literature may result in somewhat general descriptions. Thus the following summary aims to list and distinguish among possible ICT options deemed useful for this study. ICT options are categorised into individual/group and inter-organisational levels. ICT at the interorganisational level builds a necessary framework for ICT options at the individual/group level. In addition, ICT options at the individual/group level are categorised by the kind of service (exploring, informing, participating, guiding) they provide. The service types are taken from the knowledge ecology model (Wenger \& Ferguson, 2006). Whereas exploring services do not particularly focus on knowledge transfer and learning in groups, informing, participating and guiding services appear more important for this study's context. Thus the following summary only includes these three categories. 
Table 5: Summary of ICT options

\begin{tabular}{|l|ll|}
\hline Level of application & \multicolumn{1}{c|}{ ICT options } \\
\hline Inter-organisational & - & \multicolumn{1}{c|}{ Knowledge transfer management system (KTMS) } \\
level & - & Knowledge management system (KMS) \\
& - & Network infrastructures/technologies (Inter-organisational \\
& Computer Mediated Communication technologies; Extranet \\
& VPN) \\
& - E-learning system (portal solution) \\
\hline Individual/group level & - $\begin{array}{l}\text { Informing: online learning, virtual classrooms, subscription } \\
\text { service; search engine tools }\end{array}$ \\
& - $\begin{array}{l}\text { Guiding: , e-mentoring, remote coaching, } \\
\text { videoconferencing } \\
\text { - Participating: online CoP, instant messaging, email, social } \\
\text { software (wikis, blogs), discussion forum, peer-to-peer } \\
\text { applications }\end{array}$ \\
\hline
\end{tabular}

The next section describes the three theoretical models informing this research. 


\subsection{Theoretical models informing this research}

Resulting from its main goals of gaining a better understanding of how people who are working in the R\&D sector transfer knowledge within social networks and which practical implications for ICT could be drawn from this knowledge, this study is informed by various theoretical lenses. The three main perspectives of this study include the knowledge transfer and learning perspective, the social network perspective and the ICT support perspective. A consideration of multiple perspectives could thus offer a richer and more holistic study approach. Overall, a framework combining theoretical and practical approaches appears necessary to address this study's goals. Given the study's nature, there was little theory to guide the research process. Popular theories employed in IS research, such as actor network theory, tasktechnology-fit or diffusion of innovations, to name a few examples, did not seem to help achieve this study's goals. Actor network theory, with LaTour, Callon and Law (1986) as the originating authors, focuses on human and non-human actors in a network and assumes that all actors are equally powerful. Knowledge transfer structures in R\&D social networks, the main subject of this study, may include more powerful relationships and actors than others. Thus, actor network theory does not meet this study's assumptions. Task-technology-fit theory (Goodhue \& Thompson, 1995) aims to match appropriate technologies to certain tasks in order to achieve a more effective outcome of individual performance. This study aims to suggest appropriate ICT to support social relationships to achieve better knowledge transfer. However, the focus is on exploring these social relationships in the R\&D context and not to analyse the individual performance as an outcome. Thus task-technology-fit theory is not appropriate in this context. Finally, diffusion of innovation theory (Rogers, 1962) is a popular theory used in the R\&D context. This main part of this theory describes the process of the adoption of innovations. The focus of this study instead was to explore formal and informal knowledge exchange without investigating the adoption of new knowledge. Thus the diffusion of innovation theory is also not appropriate for this study's context. 
Given the foregoing, there were thus a number of theoretical lenses which could be employed in this research, in particular the social network analysis (SNA) perspective, Media Synchronicity Theory (MST) (Dennis et al., 2008) and Wenger and Ferguson's (2006) knowledge ecology model. Specifically, the social network analysis perspective that is based on key concepts of social network theory could guide the exploration of inter-organisational knowledge transfer in this study. Although this section gives a brief outline, Section 3.5 in Chapter 3 will expand on these key concepts.

In addition, MST (Dennis et al., 2008) could be employed as a theoretical lens which, in its most basic terms, views the right choice of media as a facilitator for group communication (e.g. in workshops or seminars). As this thesis also aims to draw implications for ICT to support knowledge transfer in a group learning environment (e.g. workshops), the application of this theory would be appropriate. In regard to specific media characteristics, such as reprocessability or transmission velocity, and their purpose in group communication, media can be characterised as having a high, medium or low level of synchronicity. Section 2.5.2 will expand on the key concepts of MST in relation to this thesis.

As a third perspective, Wenger and Ferguson's (2006) knowledge ecology model could guide the development of practical implications for ICT support. In comparison to MST, this model represents a more practical approach. Wenger and Ferguson's (2006) model was developed particularly to support blended learning in an organisational environment. It provides several ICT and non-ICT based knowledge services for learning. Characteristics such as informing, guiding, exploring and participating describe these services. This model has already been introduced briefly in Section 2.4.2.3., but Section 2.5.3 will provide a more detailed description.

These three theoretical perspectives - the SNA perspective, MST and the knowledge ecology model - could be applied to the context of this study with the aim to describe key knowledge transfer structures and processes and develop recommendations for 
possible ICT support. These recommendations should help study participants to facilitate collaborative learning and, in this regard, commercialisation of crucial research knowledge in the NZ R\&D sector. The following sections describe the key concepts of these three theoretical perspectives.

\subsubsection{A social network analysis (SNA) perspective}

In this thesis, SNA which uses key concepts of social network theory could be employed. In the knowledge management context, the application of SNA supports the identification and improvement of knowledge transfer in informal networks (Liebowitz, 2005). This section briefly outlines the key concepts of social network analysis (SNA).

In general, the SNA perspective focuses on social relationships that consist of a set of nodes and their ties. Nodes are the individual actors within the network, and ties are the relationships between the actors (Hanneman, 2005). Characteristics of social relationships can differ in terms of the following three aspects: content, direction, and strength (Garton et al., 1997). For example, content could be represented by goods and services, social support or knowledge; direction could be represented by advice giving and seeking activities.

In particular, tie strength, which describes the intensity of a relationship (Haythornthwaite, 1996), is a key theme in social network analysis. When two actors exchange resources, it forms a tie between them. Tie strength measures often represent either weak or strong ties. These ties can be measured by the frequency, reciprocity, emotional intensity and intimacy of that relationship (Friedkin, 1980; Granovetter, 1973).

Further, the key concept of Granovetter's (1973) weak tie theory argues that weak ties are in certain circumstances (successful job-searching) more significant than 
strong ties. Characteristics of weak ties are: infrequent maintenance, absence of emotional intensity or mutual confidence and a low reciprocity of transferring information (Granovetter, 1973).

Centrality is an additional key concept in SNA (Freeman, 1979; Valente, Coronges, Stevens \& Cousineau, 2008). The notion of 'central positions' is related to the degree of power that recipient organisations hold within a network. For example, the organisations occupying the central positions in a network are often the most powerful. They could thus control interactions of other network members. However, the connection between social structure and power remains a topic of future research (Hanneman, 2005). SNA uses centrality measurements to identify these powerful positions (e.g. organisations) in a network. Various centrality measures exist in social network analysis. Typically, the most used centrality measures are degree, closeness, betweenness, and eigenvector (Valente et al., 2008).

The social network analysis approach has been previously applied to the knowledge management context and is suitable for mapping formal and, more especially, informal knowledge transfer in a network (Anklam, 2002; Chan \& Liebowitz, 2006). Section 3.5 of Chapter 3 will expand on this description of key concepts of SNA.

\subsubsection{Media Synchronicity Theory (MST)}

Media synchronicity has been defined as "the extent to which the capabilities of a communication medium enable individuals to achieve synchronicity" (Dennis et al., 2008, p.581). Synchronicity defines "a state in which individuals are working together at the same time with a common focus" (Dennis et al., 2008, p.581). In other words, media synchronicity refers to the degree of group work on the same activity at the same time. In essence, MST relates to media capabilities and aims to identify the best possible medium to support users and their communication processes within a specific social context (Dennis et al., 2008). 
MST is a relatively new IS theory, introduced by Dennis and Valacich in 1999. It originated by building upon and improving the concept of Media Richness Theory (Daft \& Lengel, 1984). In IS research, MST has been cited frequently, for example in relation to decision support (e.g. Deluca \& Valacich, 2006; Murthy \& Kerr, 2003), technology-supported collaboration (Maruping \& Agarwal, 2004) and in communications research (Munzer \& Holmer, 2009). Maruping and Agarwal (2004), for example, investigated the appropriate assignment of technologies to group communication tasks by drawing on MST. This helped to identify functionalities of a variety of ICTs, and map these functionalities onto interpersonal processes such as conflict management and motivation and confidence building.

Munzer and Holmer (2009) explored the effects of media synchronicity for problemsolving tasks in groups. Therefore, they focussed on investigating media characteristics such as parallelism, immediacy of feedback, and reprocessability employed in MST. Unexpectedly, their findings indicate asynchronous media characteristics, such as a high reprocessability and a low immediacy of feedback, support a high engagement in discussion and information sharing. Applying MST, this would be rather an expected result for synchronous media characteristics.

The following media capabilities are considered in MST:

\section{1) Transmission velocity}

Transmission velocity describes a medium's speed of delivering a message to the recipient (e.g. the knowledge seeker) (Dennis et al., 2008). Dependent on the medium and the use of this medium, transmission velocity has high (e.g. videoconferencing), medium (e.g. instant messaging) or low synchronicity (e.g. documents) (Dennis et al., 2008). Media with a high transmission velocity allows the knowledge giver to quickly transfer knowledge, and, in turn, the knowledge seeker to benefit from a quick response. 


\section{2) Parallelism}

Parallelism describes the extent to which information from several senders can be transmitted over the medium at the same time (Dennis et al., 2008). Traditional media such as the telephone allow few transmissions to take place over the medium simultaneously. This limits the amount of information that can be transmitted in a certain time period (Dennis et al., 2008). On the other hand, many of the new media, such as conferencing tools, can be configured to support many simultaneous transmissions. This increases the amount of information that can be transmitted in a given time period (Burgoon, Bonito, Bengtsson, Ramirez, Dunbar \& Miczo, 1999-2000).

\section{3) Symbol Sets}

The ability to use multiple ways of communication with text, voice and/or pictures allows users to express themselves in a variety of ways which are not limited to text. These features are supported by forms of multimedia or face-toface communication. Examples could be video-conferencing and face-to-face meetings.

\section{4) Rehearsability}

The ability to edit information before transfer gives the user the freedom to properly prepare or correct a message before sending it. A knowledge giver may want to add another explanation or change an example before sending it to the knowledge seeker. Several ICT-based media support this feature, for example email, discussion forums and social software. Other media such as videoconferencing tools or telephones cannot provide this feature. 


\section{5) Reprocessability}

The ability to save information for later reuse enables the user to save information, for example emails, voice messages or discussion forum threads. This is a particularly significant characteristic in the context of knowledge reuse. Knowledge seekers may want to save knowledge that they received earlier in order to come back to it at a later stage or to provide this knowledge to another seeker.

Dennis et al. (2008) argue that these media capabilities are configurable and need to be considered in relation to the context in order to decide which of many capabilities of one medium "is more or less important in a given situation" (Dennis \& Valacich, 1999, p.3). The key to effective use of media is to match media characteristics to certain communication processes required to perform a task involved with member support, group well-being and production.

Moreover, MST assumes that group communication is based on two communication processes: conveyance and convergence (Dennis et al., 2008). Convergence describes reaching a mutual understanding/consensus. Conveyance refers to the actual physical transfer/transmission of information. For group communication processes that rely on convergence, the use of media providing high synchronicity (e.g. high transmission velocity and low parallelism) will lead to better performance (Dennis et al., 2008). Whereas for group communication processes that aim for conveyance, the application of media providing low media synchronicity (e.g. low transmission velocity and high parallelism) will lead to better performance (Dennis et al., 2008).

Media such as email, video-conferencing or instant-messaging show diverse levels of synchronicity. For example, a video-conference tool allows for a synchronous and quick transfer of knowledge (transmission velocity) and therefore has high synchronicity (Dennis et al., 2008). In contrast, email represents an asynchronous medium for transferring knowledge, thus its transmission velocity was rated low to medium. This reflects a low level of synchronicity (Dennis et al., 2008). To support 
both low and high synchronicity for achieving an effective communication process, Dennis et al. (2008) recommend a mix of diverse media.

In another context, Kerres and DeWitt (2003) suggest the use of synchronous media, such as chat, for the early stages of group building, as this type of media may help to foster a sense of community. In contrast, asynchronous media are more suitable to facilitate group communication in subsequent phases and well-established groups. Therefore, a mix of synchronous and asynchronous media may be best suited to support group communication over time. This recommended mix of media confirms Dennis et al.'s (2008) suggestion that the “best medium for a given situation may be a combination of media" (Shahriza, Karim \& Heckman, 2005; Watson-Manheim and Belanger, 2007 cited in Dennis et al., 2008, p.588).

\subsubsection{The knowledge ecology model}

As a third possible theoretical lens for this study the knowledge ecology model for blended learning (Wenger \& Ferguson, 2006) is outlined. A brief introduction of this model has already been given in Section 2.4.2.2. The development of this emergent model was driven by practical purposes in order to facilitate Sun Microsystems' various training services. The knowledge ecology model is the outcome of combined education and training efforts with knowledge management initiatives. "The resulting knowledge ecosystem creates an environment in which people are provided opportunities to gain knowledge or learning through methods and models that best support their needs, interests, personal situations, and individual learning styles" (Wenger \& Ferguson, 2006, p.87), including computer-mediated options, such as e-

books, online communities of practice, discussion forums and chat, web-conferencing and e-mentoring.

Wenger and Ferguson's (2006) model, which is illustrated in Table 6, provides a range of knowledge services to people who want to learn in different ways. Further, 
the model does not automatically define the media for delivery and therefore provides insights into the possible use of ICT to support these knowledge services.

Table 6: Knowledge services (adapted from Wenger \& Ferguson, 2006, p.89)

\begin{tabular}{|c|c|}
\hline Exploring & Participating \\
- Content repositories & - Online communities of practice \\
- Learning content management & - Discussion forums and chat \\
- Performance support & - Talent directories \\
- Resource centres & - Learning communities \\
- Learning portals & - Instant messaging \\
- E-books & \\
\hline Informing & Guiding \\
- Continuous learning & - Collaboration \\
- Skills assessment & - E-mentoring or remote coaching \\
- Pretests & - Web conferencing \\
- Online learning & - Simulations \\
- Virtual classrooms & - Skills management \\
- Blended learning & - Interaction with experts \\
- Online certification & - Online labs \\
- Learning management & \\
\hline
\end{tabular}

Four distinct groups of knowledge services were identified in the model: (1) exploring, (2) participating, (3) informing, and (4) guiding. People explore resources for ideas and practical insights. ICT examples which belong to the exploring category include e-books and learning portals. People participate in a community to create understanding (Wenger \& Ferguson 2006). Online CoP and instant messaging are ICT examples that aim to support participation in a group. Further, experts inform people about common theories and practice, and they also guide people as they apply their knowledge and skills to a new situation (Wenger \& Ferguson 2006). ICT in the knowledge service category of informing can consist of online learning and virtual classrooms. E-mentoring, remote coaching and web conferencing are examples of guiding knowledge services.

Although this model provides a good background in identifying knowledge services for various learning situations in the workplace, it also includes some drawbacks. It may be difficult to decide whether some knowledge services belong to only one group of services. For example, an e-book listed under exploring services may also have informing characteristics. Another example could be discussion forums. A 
forum may be provided mainly for participating purposes, but some people may only want to use it for receiving information.

Due to its diverse knowledge services, Wenger and Ferguson's (2006) model may also support a mix of diverse media for specific communication scenarios, for example collaborative learning. Collaborative learning could employ face-to-face workshops and ICT-based media to facilitate group communication. This approach appears particularly suitable for the context of identifying additional learning services for members of social networks embedded in a larger R\&D network.

Services such as online communities of practice, interaction with experts or learning portals may be useful to facilitate collaborative learning. In the knowledge ecology model, these services involve participating, guiding or exploring. Whereas participating and guiding services may require a high transmission velocity, the exploring services would have less need for that. However, the exploring services would benefit from media providing multiple ways of communication with text, voice and/or pictures. This need for diverse media characteristics would suggest a switch between media to best support collaborative learning.

\subsubsection{Summary}

The application of these three theoretical lenses - the SNA perspective, Media Synchronicity Theory and the knowledge ecology model - would align well with the goals of this research to investigate possible ICT support for specific knowledge transfer structures and processes. As this study focussed on exploring interorganisational knowledge transfer, the major informing theoretical concepts could be drawn from the SNA perspective. This approach has been previously employed in the knowledge management context and is suitable for the identification of formal and informal knowledge transfer in a network (Anklam, 2002; Chan \& Liebowitz, 2006). Thus it also appears appropriate for exploring knowledge transfer structures in the context of advice giving and seeking activities in this study. Key knowledge transfer 
structures and processes might then help develop implications for ICT support. Media synchronicity theory focuses on the appropriate use of ICT for group communication dependent on specific media characteristics and communication processes. This theory has been previously applied in other studies to investigate technologysupported collaboration (Maruping \& Agarwal, 2004) or communications (Munzer \& Holmer, 2009). Thus this theory might help to connect key knowledge transfer structures and processes in social networks to possible ICT support.

In addition, the knowledge ecology model could also inform the development of implications for ICT, particularly for an inter-organisational learning context. The knowledge ecology model is a practical framework that combines ICT and non-ICT options to provide a suitable learning environment in which knowledge can be transferred.

\subsection{Chapter Summary}

This chapter has summarised a range of literature at the intersection of four key themes relevant to this study: inter-organisational knowledge transfer, R\&D networks, knowledge transfer in social networks and the use of ICT support. Further, this chapter has described the key theoretical concepts informing this research.

In the field of knowledge transfer in R\&D networks many researchers have focussed on factors such as management, trust, organisational culture, and strategic goals that affect knowledge transfer. Nevertheless, there is a lack of research addressing the issue of how knowledge transfer is carried out in the inter-organisational environment and how it can be supported by ICT. The literature review has established a gap in the literature in the area of applying a social network perspective to knowledge transfer among organisations, and more specifically to the inter-organisational R\&D context. 
In particular, inter-organisational knowledge transfer structures and processes related to the strength of relationships between individuals' informal key roles in the knowledge management context have not been researched before. This includes research on their knowledge transfer characteristics, such as the types of knowledge they exchange and the directions of knowledge transfer. Further, social relations and organisational boundaries related to knowledge transfer have been identified as key emerging themes in knowledge management (Argote et al., 2003).

The literature review has illustrated the current research into ICT, specifically social or collaborative technologies in relation to knowledge transfer. This review revealed that there has been little research into ICMC infrastructures that are able to support inter-organisational social networks and their collaborative characteristics.

As the key concepts informing this research, the social network analysis (SNA) perspective, Media Synchronicity Theory and the knowledge ecology model are employed. This combination of approaches aims to inform this study on two levels: firstly, for the investigation of inter-organisational knowledge transfer structures and processes; and secondly, for the development of implications for appropriate ICT support. Further, the outcomes from using this approach should benefit researchers as well as practitioners.

In summary, the literature review has revealed some major gaps in the existing literature around the inter-organisational environment for knowledge transfer and its ICT support. These gaps were addressed by the research question:

What are the knowledge transfer structures and processes among organisations within an $R \& D$ network? 
To allow a more detailed investigation, this question was subdivided into three subquestions:

1) What are the key structural properties of advice networks and their implications for knowledge transfer?

2) Which types of knowledge are transferred in the advice networks?

3) How can ICT support these knowledge transfer structures and processes? 


\section{The Research: Paradigm, Strategy and Design}

\subsection{Introduction}

This chapter gives an overview of the research paradigm, strategy and design employed. An integrated approach of interpretive and positivist philosophical stances was adopted for this study. In addition, a sequential embedded mixed methods research design, including qualitative and quantitative phases, was applied. This design included case research as the leading research strategy comprising a multiple case study with three sequential cases. Data collection in this case research was conducted using observations, interviews, an online survey and online follow-up questions. Key concepts of social network analysis (SNA) were embedded in the case research.

\subsection{Philosophical considerations}

The research paradigm, which underpins different research perspectives, describes the actual complexity of philosophical worldviews in a simplified manner. The paradigm allows the researcher to identify and communicate specific perspectives and assumptions (Guba \& Lincoln, 1994). In fact, the nature of the particular paradigm which guides research projects is influenced by a number of philosophical and practical considerations (Rabinowitz \& Weseen, 1997). This can include an interpretive and/or positivist philosophical stance. Traditionally, researchers working with one of these two stances approach theory development differently. In the positivist stance, theories are mostly validated through data gathered by quantitative research methodologies. In the interpretivist stance, theory is emergent and follows rather than precedes research.

In keeping with this study's research goals an integrated approach of interpretive and positivist philosophical stances was adopted. This study aimed to understand 
knowledge transfer among $\mathrm{R} \& \mathrm{D}$ organisations. It sought to generate insight into inter-organisational social networks, which indicates an interpretive stance (Klein \& Myers, 1999). In particular, the research aimed to understand how interorganisational social networks work, the process of knowledge transfer among network participants and how they could be supported by suitable ICT solutions. This part of the research views meaning as subjective and socially constructed through human interactions.

Nevertheless, this research included the identification and analysis of knowledge transfer structures and processes. Knowledge transfer structures consist of social relationships and are based on the theoretical concepts of social network research, which measures tie strength by frequency of contact, for example. These concepts presume that reality is objectively given and measurable and thus follow a positivist epistemology (Orlikowski \& Baroudi, 1991).

Theory informed large parts of this study and helped develop the research methods. In addition, recommendations (propositions) on appropriate ICT support for interorganisational knowledge transfer were derived from the key analysis results and thus emerged throughout the study. Therefore, theory preceded, and also emerged from, this study's research. This goes along with Morgan's (2007) recent statement about the application of mixed methods research: "Yet any experienced researcher knows that the actual process of moving between theory and data never operates in only one direction" (Morgan, 2007, p.70).

Moreover, Lee (1989) suggests that the interpretive and positivist stances provide diverse methods that can be combined in a way that strengthens the research results. Such an integrated approach of positivist and interpretive philosophical stances was adopted for this study to best achieve the research goals: understanding the context of inter-organisational knowledge transfer in social networks in the R\&D sector including the measurement of knowledge transfer structures within these networks with the aim to provide recommendations on appropriate ICT support. 
In keeping with Hirschheim and Klein's (1989) view of methodological pluralism, a mixed methods approach was employed for this study to combine the suitable assumptions and perspectives of this research. A mixed methods approach combines both qualitative and quantitative methods. Alongside qualitative and quantitative research, it has been recognised as the third research paradigm (Johnson et al., 2004). Section 3.4 will expand on this study's mixed methods research. The following section firstly describes the case research conducted.

\subsection{Case research}

Case research was the leading strategy for this study. Firstly, an overview of case research is given. Then, this case research strategy and the unit of analysis are described. The three cases in this multiple case study were conducted sequentially. Each case comprised three sequential research phases: Phase 1 (qualitative), Phase 2 (quantitative) and Phase 3 (qualitative). The overall research focus was on investigating inter-organisational social interactions related to knowledge transfer and ICT support.

\subsubsection{An overview of case research}

Case research is a research strategy that aims to understand complex social phenomena in their real-life context (Yin, 2003). "The researcher explores in depth a program, an event, an activity, a process, or one or more individuals" (Creswell, 2003, p.15). Case research has some distinctive key characteristics. Firstly, it investigates phenomena within their natural settings (Benbasat, Goldstein \& Mead, 1987). Secondly, it focuses on contemporary events, and it is also the most appropriate way to address 'why' and 'how' questions. Moreover, it shows flexibility in terms of site selection and methods of data collection as the research progresses. Finally, case research allows study of the unit of analysis in its full complexity, and 
the research is typically not carried out under controlled conditions (Benbasat et al., 1987).

A case study is recommended for research into areas in which little previous work has been done (Benbasat et al., 1987). It has been a common research strategy in various fields such as psychology, sociology, political science, social work, business, and community planning (Yin, 2003, p.1). In addition, the case research strategy's holistic nature makes it particularly well-suited to the IS field, as it aims to understand the complex interactions between organisations, technologies and people (Dubé \& Paré, 2003).

The ideal case research approach uses a methodological stance that best suits the investigation of a particular research problem or question (Yin, 2003). A case study can use qualitative, quantitative or mixed methods approaches. Qualitative methods in case research provide a richness of data description that would be lost in a quantitative analysis (Yin, 2003). However, "the case study strategy should not be confused with 'qualitative research" " (Denzin \& Lincoln, 1994).

Multiple data collection methods, including qualitative and quantitative methods, are ideally part of case study research (Dubé \& Paré, 2003). This helps to cover a wider scope and may provide a fuller picture of the phenomenon under study. Yin (2003) identifies six sources of qualitative evidence in case research. These are documentation, archival records, interviews, direct observation, participant observation and physical artefacts. These methods help to explore reality in greater detail and consider more variables than would be possible using other approaches (Galliers, 1992).

Besides the purpose of exploration, case study research is widely used for hypothesis generation (Benbasat et al., 1987). In contrast, Yin (1993) points out that case study research can also take advantage of the previous construction of theoretical propositions. Useful skills for the case researcher include the willingness and ability 
to ask good questions, listen well, adapt to changes and accept contrary results during the data gathering process (Yin, 2003).

Case study research can be carried out using either a single or multiple case study design. A single case study is considered to be appropriate for research that investigates one unique case or tests an established theory. Moreover, it is well-suited for a research problem that is exploratory in nature (Yin, 2003). Case study research has been criticised, especially regarding its lack of generalisability. Although multiple case studies have the disadvantage of requiring extensive resources and time, they help to overcome this difficulty and improve transferability (Miles \& Huberman, 1994). Multiple case studies also provide a better data triangulation (Yin, 2003), and offer a better data foundation that can be more easily replicated (Yin, 1993). Moreover, "the evidence from multiple cases is often considered more compelling, and the overall study is therefore regarded as being more robust" (Herriot \& Firestone, 1983 cited in Yin, 2003, p.46).

The following section describes this study's case research in detail, including the setup of the multiple case study, recruiting of study participants for data collection, the three social networks and the unit of analysis.

\subsubsection{This case research}

This case research comprised three cases that were examined consecutively in a multiple case study. Each case represented a topic-based, inter-organisational social network among R\&D organisations in New Zealand. Each network consisted of members from a range of organisations, such as private businesses (mainly research and consulting firms), Crown Research Institutes (CRIs), central and local government, universities and non-profit organisations. Members of each social network shared a general willingness to exchange knowledge. They were working, and therefore interested, in the same professional field: hydrology, marine biology or 
coastal hazards. The social networks were based on informal relationships. People in these informal relationships sought professional advice from peers or gave professional advice to other peers during the last year. The structural design of this multiple case study is illustrated in Figure 5.

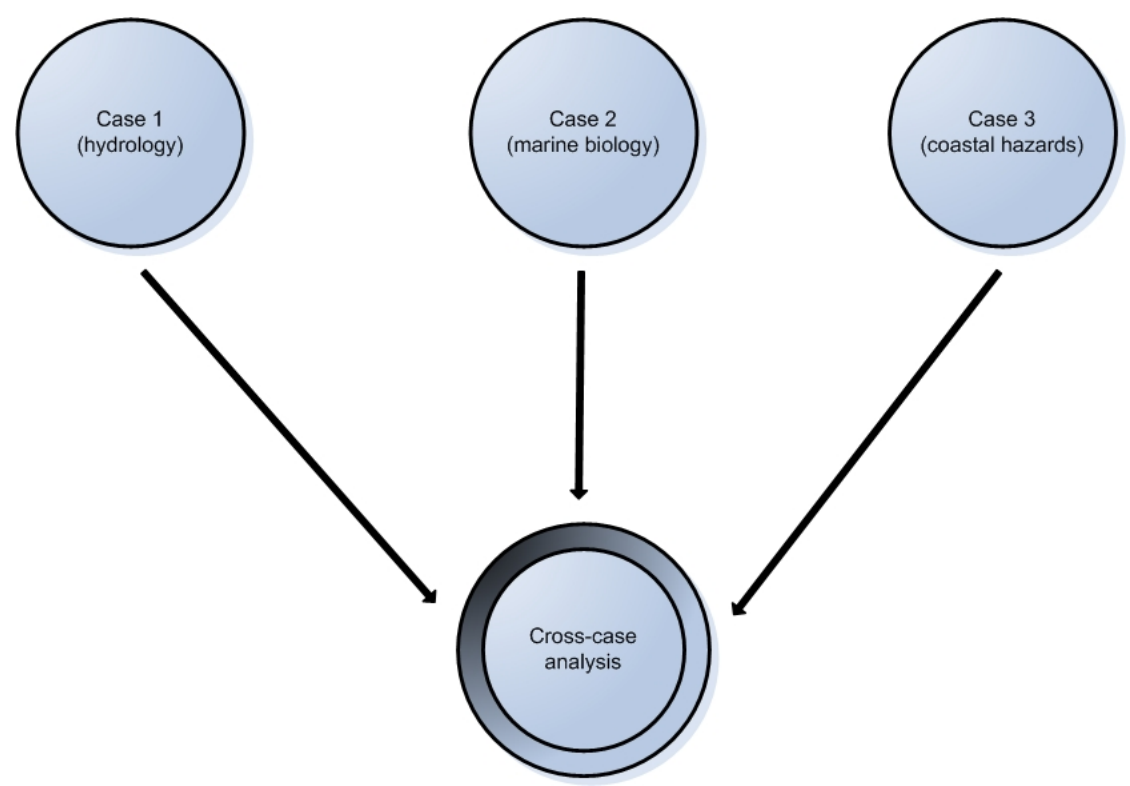

Figure 5: Multiple case study design

Figure 5 illustrates the three sequential cases and a final cross-case analysis. Case 1 was firstly conducted as a pilot case study. Lessons learned were integrated into the data collection for the subsequent Cases 2 and 3. Case 1 represented a social network in the professional field of hydrology. Case 2 was a social network in the marine biology profession. Case 3 represented a social network in the professional field of coastal hazards. A final cross-case analysis compared results from the three cases. Procedures undertaken in this cross-case analysis are described in Section 3.9. ${ }^{2}$

Recruiting study participants

Recruitment of study participants was conducted prior to data collection in each of the three cases. Potential participants were formally invited to take part in this study.

${ }^{2}$ See page 113 
In addition, two meetings were organised with a participating key organisation that provided workshops/seminars to the public. In these meetings the study outline was presented and possible issues were discussed. A research proposal outline was provided to this key organisation describing the goals and benefits of the study as well as the study approach. It was agreed to provide this organisation with a business report outlining key results at the end of this study. The feedback on this report was integrated in the study results.

Throughout the year, the contacted key organisation offers context-specific face-toface workshops to the public. The three workshops used for data collection in the three cases of this multiple case study were selected in conjunction with the training manager of this organisation in the period between November 2007 and July 2008. Purposive sampling techniques were employed in the selection to ensure comparability of the cases (Teddlie \& Yu, 2007).

All the workshops were held face-to-face in groups and had between seven and 22 participants from diverse groups of organisations. A high diversity in organisational affiliation of participants was particularly sought after to provide a good representation of the respective environmental sectors. In each workshop, which was the point of departure for data collection in each case, the trainer briefly explained the researcher's appearance as an observer and asked the workshop participants to take part in the follow-up data collection for this study. Most of the participants did not know each other before the workshop. The trainer was also asked to select a variety of important organisations in the respective environmental sectors in New Zealand for data collection in Phase 2. In order to achieve a more appropriate sample for each social network case, individual members from associated professional societies in New Zealand were also invited to participate in the online survey in Phase 2.

In order to meet Victoria University of Wellington's ethical standards, this research involving the investigation of human participants had to be approved by the University before data collection was undertaken. In each case, workshop participants 
were asked to take part in the follow-up data collection including interviews (Case 1 only), an online survey and follow-up questions on survey results provided electronically in the form of a Wiki tool.

Due to the social network's informal nature and the voluntary participation of members in each case study, the number of participants in each social network could not be controlled and varied between 52 and 81 . However, clear boundaries could be drawn around each social network case. Each network included members of topicspecific professional societies in New Zealand. These societies represent a common interest group in a specific professional topic area: hydrology, marine biology or coastal hazards. In all cases, study participants were also from a variety of organisations such as Crown Research Institutes (CRIs), private businesses, local and central government and universities. The following table shows the numbers of study participants by case and method.

Table 7: Overall numbers of study participants

\begin{tabular}{|l|c|c|c|}
\hline Data collection method & $\begin{array}{c}\text { Case 1 } \\
\text { (hydrology) }\end{array}$ & $\begin{array}{c}\text { Case 2 } \\
\text { (marine } \\
\text { biology) }\end{array}$ & $\begin{array}{c}\text { Case 3 } \\
\text { (coastal } \\
\text { hazards) }\end{array}$ \\
\hline Workshop participants (observation) & 7 & 16 & 22 \\
\hline $\begin{array}{l}\text { Interviews with workshop } \\
\text { participants/trainer }\end{array}$ & 6 & - & - \\
\hline Online survey & 80 & 81 & 52 \\
\hline $\begin{array}{l}\text { Follow-up questions (workshop } \\
\text { participants) }\end{array}$ & 2 & 5 & 3 \\
\hline
\end{tabular}

\subsubsection{The unit of analysis}

In case research, decisions on the unit of analysis are largely based on research purposes and questions (Yin, 2003). The social phenomenon needs to be clearly defined in relation to its contextual boundaries (Miles \& Huberman, 1984; Yin, 2003). This study aimed to examine the nature of knowledge transfer, particularly 
processes and structures as well as media use and ICT support, within interorganisational social networks in the environmental R\&D sector. As these social networks and associated methods and concepts are constituted by the structure of their relationships, this study defined the social relationship as its unit of analysis.

According to SNA literature, the social relationships can be distinguished according to the level of analysis (Scott, 2000). This may be the individual, group, organisational or even a national level (Mizruchi \& Marquis, 2006). In keeping with this study's goals, the social relationship was investigated at the inter-organisational level. This level particularly looked at diverse groups of organisation that were communicating with each other. Nevertheless, implications for ICT support were also drawn at the individual and group level to address specific supportive tools for knowledge transfer among individuals and within groups as part of the larger interorganisational R\&D networks.

\subsubsection{Summary}

This section described the case research framework used in this study. It outlined background information on case research and described the application of case research to this study. Then, the multiple case study conducted within this framework was explained. The multiple case study comprised three cases. For each case, a qualitative data collection phase (Phase 1) was followed by a quantitative phase (Phase 2). Subsequently, the second quantitative phase was followed by a third and final qualitative phase (Phase 3). These three phases are described in detail later in this chapter. In addition, the recruitment of the study participants was outlined. As SNA methods and concepts rely on the structure of social relationships, the unit of analysis was defined as the social relationship at an inter-organisational level in this study. Section 3.4 reports on mixed methods research in general, and the mixed methods design of this study in particular. 


\subsection{Mixed Methods Research}

This section gives an introduction to mixed methods research and outlines its characteristics and possible designs. Then, a description of the research design used for this study follows.

\subsubsection{The nature of mixed methods research}

Mixed methods research is a combination of qualitative and quantitative research strategies. A classic case of mixed methods research involves employing case studies together with mailed surveys. Case studies, for example, give greater depth, while surveys give greater breadth (Tashakkori \& Teddlie, 1998). In addition, studies can employ a mixed methods approach in order to investigate a phenomenon from diverse perspectives (Creswell, 2003). The researcher is able to develop a better understanding of the research context and therefore tends to produce better results. According to Mingers (2001), the application of mixed research methods, "preferably from different (existing) paradigms" (p.240), results in even richer and more reliable findings. Particular research methods may also be more appropriate for certain contexts, thus a combination of approaches may lead to a more comprehensive research outcome.

However, mixed methods approaches have been criticised because of their different underlying epistemological assumptions (Creswell, 2003). In this regard, Mingers (2001) names philosophical feasibility, which refers to paradigm incommensurability, cultural feasibility, cognitive barriers expressed by psychological feasibility and other practical barriers as issues that need further research. As outlined earlier, this study employed an integrated approach of interpretive and positivist philosophical stances. Although, some critical aspects as described above remain, this approach helps accommodate the study's diverse perspectives which may lead to more comprehensive research findings. In addition, the diverse quality assurance activities undertaken, as outlined in Section 9.2, aim to help reduce critical aspects of feasibility. 
Kelle (2006) suggests five advantages a mixed methods approach might have: (1) based on a sequential quantitative-qualitative study, the quantitative part can help to select cases for the qualitative research part; (2) results from qualitative research can reveal heterogeneity in quantitative data and unexplained variables and mis-specified models; (3) qualitative findings can help to explain incomprehensible statistical findings; (4) the qualitative part of a mixed methods approach can discover a lack of validity of quantitative measurements and (5) the quantitative part could corroborate results from the qualitative research part in order to transfer these findings to other domains.

Articles that recommend a mixed methods approach have been published in highly ranked IS journals such as Information Systems Research (e.g. Mingers, 2001), the Journal of Information Technology (e.g. Galliers, 1993) and MIS Quarterly (e.g. Kaplan \& Duchon, 1988). Further, an increasing number of IS studies that employ a mixed methods approach are appearing in IS journals such as Organization Science (e.g. Markus, 1994) and MIS Quarterly (e.g. Ang \& Slaughter, 2001; Trauth \& Jessup, 2000). In 2001, Mingers (2001) calculated that 20\% of IS research studies were using this approach. Moreover, the launch of an international journal, the Journal of Mixed Methods Research, in 2007 has indicated the growing interest in mixed methods research (Hooper, 2006).

The combination of methods used in mixed methods research has been described in a number of ways. These have included 'parallel', 'dominant', 'embedded', and 'multimethodological' (e.g. Creswell, 2007; Mingers, 2001; Tashakkori \& Teddlie, 1998). However, three main types of mixed methods have been identified (Hooper, 2006):

- sequential mixed methods research, in which the researcher aims to expand or elaborate on the results of the first method, by using the second; 
- concurrent mixed methods research, in which both qualitative and quantitative methods are used together and interpreted in an integrated manner; this is also called the triangulation design; and

- transformative mixed methods research, in which the researcher employs an overarching theoretical perspective (Creswell, 2003).

\subsubsection{The mixed methods design of this study}

This study's goal was to combine advantages from both qualitative and quantitative perspectives: a qualitative in-depth study of a social phenomenon, the interorganisational social network in an R\&D environment, and the ability to provide transferability of findings to social networks in other environments. To best explore and explain this research environment, the research design of this study used a sequential mixed methods strategy. For each case, a sequential design with three subsequent phases (Phases 1,2 and 3) was chosen to combine both exploratory and explanatory aspects in a dominant qualitative research approach. The particular social and institutional context of participants in a study can be represented most efficiently with textual data (Kaplan \& Maxwell, 1994). In addition, the quantitative SNA part was embedded in the qualitative case research and provided more detailed information on the structural properties of the inter-organisational knowledge transfer.

Phase 1 provided the researcher with the opportunity to learn about the contextual setting including individual study participants and organisations. This design was employed, as it allowed the researcher to apply the results of Phase 1 to the development of the data collection instrument for the second phase. In Phase 2, an online survey was distributed to potential study participants from diverse organisations. Finally, in Phase 3, follow-up questions helped explain and confirm key results of Phase 2. This three-phase sequential approach combined the advantages of the two possible sequential two-phase approaches: the exploratory design (Phase 1 
- Phase 2) and the explanatory design (Phase 2 - Phase 3). The three phases of data collection are described in more detail in Sections 3.6 (Phase 1), 3.7 (Phase 2) and 3.8 (Phase 3).

In keeping with this study's goals, the 3-phase mixed methods approach included decisions about timing, weighting and mixing of qualitative and quantitative approaches. The following decision tree (adapted from Creswell (2007)) illustrates these decisions.
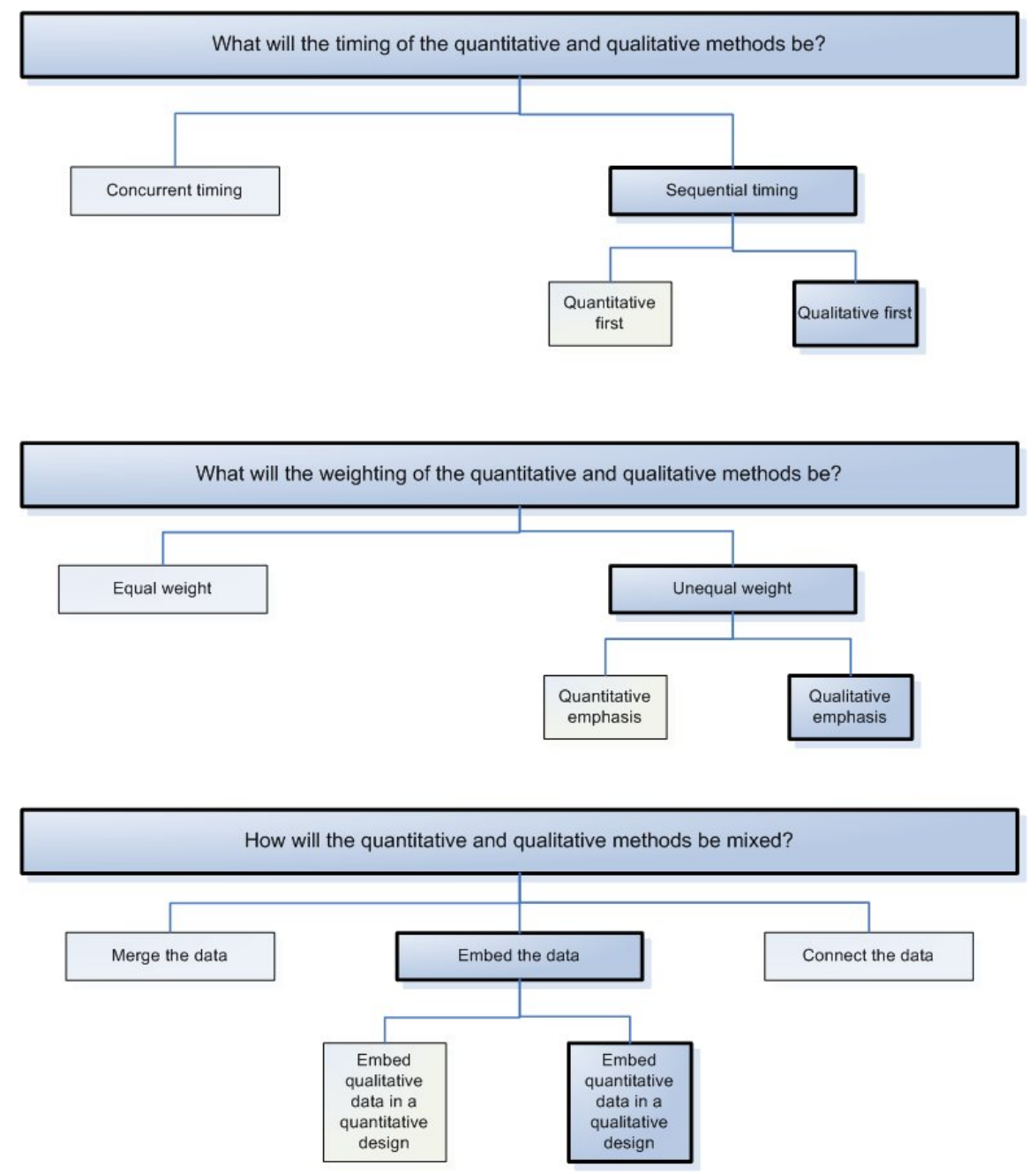

Figure 6: Decision tree for mixed methods design criteria for timing, weighting, and mixing (adapted from Creswell, 2007, p.80) 
The decision tree illustrates the sequential (timing), dominant (weighting) and embedded (mixing) aspects of this design. As already outlined, the quantitative SNA phase (Phase 2) is embedded within two qualitative phases (Phases 1 and 3). The emphasis was on the qualitative approach, as the majority of data collection phases (Phases 1 and 3) were qualitative in nature. In addition, the qualitative methods (observation, interviews and open-ended follow-up questions) outnumbered the quantitative methods (online survey).

\subsubsection{Summary}

This section described the nature of mixed methods research, its advantages and downsides. The study's sequential mixed methods design was described. This 3phase design approach was explained and illustrated - a dominant qualitative framework (Phases 1 and 3) with an embedded quantitative phase (Phase 2).

Section 3.5 will report on the key concepts of SNA employed in Phase 2.

\subsection{Quantitative research with Social Network Analysis}

This section outlines key concepts of social network analysis (SNA) that were applied to this study. SNA typically analyses social relationships in diverse contexts ranging from disease-spreading research to terrorist networks. The application of SNA to the knowledge management context helps identify and better understand knowledge transfer in informal networks (Liebowitz, 2005). SNA methods and techniques are based on mathematical concepts of graph theory. The SNA perspective guided data collection and analysis procedures in Phase 2 of the sequential research design which is described in Section $3.7^{3}$

\footnotetext{
${ }^{3}$ See page 102
} 
Key concepts of SNA applied to this study

The SNA perspective focuses on social relationships and views them as consisting of a set of nodes and their ties. Nodes are the individual actors within the network, and ties are the relationships between the actors (Hanneman, 2005). Social networks can be analysed either as ego-centred networks, which focus on relationships from one focal organisation or individual (ego) to others (alters) in the network, or whole networks, whereby the analysis tries to describe all the relationships between the network actors within given boundaries (Garton et al., 1997). Thus, these two network perspectives typically guide data collection in regard to the network actors considered.

This study applied the whole network perspective to investigate all relationships in each topic-based social network. This whole network approach allowed for the identification of the most influential nodes in each one of the three social networks (Cases 1, 2 and 3). These nodes (organisations) may have the influence to make decisions on ICT-based inter-organisational knowledge transfer and learning initiatives in the network.

The following paragraphs outline possible levels of analysis in SNA, properties of social relationships (content, direction and strength), the weak tie theory and the key concept of centrality.

\section{Levels of analysis}

There can be various types of relationships in a social network. Four specific relationships, differentiated according to the number of actors involved, can be identified: dyads, triads, subgroups and groups. Dyads link two actors and triads are relationships between a subset of three actors. A subgroup refers to more than three actors, whereas a group is a finite collection of subgroups and actors all of which are 
related to one another (Wasserman \& Faust, 1994). These relationships can then be studied at a range of levels such as individual, group or organisational levels. At an individual level, nodes represent individuals. At a group level, nodes are individuals who represent a group. At an organisational level, nodes (individuals) represent an organisation.

As this study was specifically investigating inter-organisational knowledge transfer in social networks, relationships were explored at an inter-organisational level. In particular, a level investigating diverse groups of organisations (inter-groups of organisations) was employed to provide comparable networks for a cross-case analysis regarding network characteristics such as size (number of relationships) and types of members. Each node represented one specific group of organisation such as central or local government, private businesses, CRIs or universities. Moreover, the dyadic level was employed, as it allows for the investigation of relationship properties, such as tie strength, between two organisations. This helped explore important knowledge transfer structures.

According to the relationships represented by a subgroup, Scott (2000) distinguishes between cliques and clusters, which are both specific types of subgroups. A clique is a subgroup in which each actor is directly related to another actor. For example, within a clique of 50 participants, each member would be directly connected to 49 other members. In contrast to cliques, clusters are subgroups that are based on the idea of similar relational or attributive data (Scott, 2000), but they do not have to be directly connected. In SNA, a cluster of 50 actors would consist of people who share the same topic-based work responsibilities, for example working on coastal hazards topics.

In a SNA diagram, actors who belong to a cluster are illustrated by an "area of relatively high density in a graph" (Scott, 2000, p.127). Local clusters in a network can also be supported by a high clustering coefficient, which is a cohesion measurement in SNA. Cohesion can be measured by the density of a network that 
looks at how well all nodes in a network are connected with each other. Local clustering and density were investigated in this study's social networks in order to depict specific network structures.

\section{Properties of social relationships}

Relational ties (social relationships) in social networks may differ in (1) content, (2) direction, and (3) strength (Garton et al., 1997). Firstly, the content of a relation refers to the resource that is exchanged, such as information, goods and services, and financial support (Garton et al., 1997; Haythornthwaite, 1999). In this study, participants communicated about topic-related matters (hydrology, marine biology or coastal hazards) on a regular basis. Thus the resource that was exchanged referred to knowledge about these topic-related matters. However, communication about administrative, personal or social matters might have also been exchanged. In addition, the content, and thus the relational tie, can differ according to the type of knowledge, for example complex or non-complex knowledge, that is exchanged (Hansen, 1999).

This study differentiated knowledge by the following characteristics: electronic availability, degree of formalising, type of existence (explicit or tacit/non-codified), representation as facts, how-to or meta-knowledge (e.g. references to other sources of knowledge). Thus, relationships could be characterised by the type of knowledge exchanged.

Secondly, a relation can either be directed or undirected which depends on the direction of transferred knowledge. Direction indicates the flow of knowledge from one actor to another. As an example, the relationship between two actors would be directed from actor $\mathrm{A}$ to actor $\mathrm{B}$, if actor $\mathrm{A}$ gave advice directly to actor B, but did not receive any advice in return from actor B. A relationship can also be undirected in nature. An example of an undirected relationship might be that of two actors who are 
friends and communicate in close physical proximity, but no specific identifiable work-related knowledge is exchanged between them.

This study considered the direction of knowledge transfer and therefore the directed social relations. The direction of knowledge transfer was differentiated by giving advice to peers and seeking advice from peers in the workplace.

Thirdly, tie strength indicates the third property of a social relationship besides content and direction. It describes the intensity of a relationship (Haythornthwaite, 1996). Tie strength is a key concept of the SNA approach. When two actors exchange resources, it forms a tie between them which can be measured as either a weak or a strong relationship. Tie strength is defined as a "combination of the amount of time, the emotional intensity, the intimacy (mutual confiding) and reciprocal services which characterize the tie" (Granovetter, 1973, p1361). These four elements are related to four indicators, closeness, duration and frequency, breadth of topics and mutual confiding, which are components of tie strength (Petróczi et al., 2007). For example, a higher frequency of contacts represents a stronger relationship. The measurement of tie strength by the frequency of contact has been used by other authors such as Granovetter (1974) and Lin, Dayton and Greenwald (1978). In particular, it has been a typical indicator of tie strength in computer-mediated communication networks (Garton et al., 1997). Other contextual contingencies, which are related to tie strength but are no components of it, are regarded as predictors. These include neighbourhood, affiliation, similar socio-economic status, workplace and occupation prestige.

Reciprocity refers to a relationship that has been confirmed by both actors. This represents a strong relationship, whereas relationships that have been reported by only one actor are unconfirmed and therefore weak in nature (Foster-Fishman et al., 2001). In addition, confirmed linkages increase the reliability of social network data (Foster-Fishman, Salem \& Allen, 2001). Various studies have highlighted the importance of reciprocity for tie strength and knowledge transfer (e.g. Bresman, 
Birkinshaw \& Nobel, 1999; Friedkin, 1980; Granovetter, 1973; Hansen, 1999; Petróczi et al., 2007).

Emotional intensity may be, for example, expressed by one actor's use of emotional language when talking about a relationship with another actor. High emotional intensity when reporting a relationship may indicate a strong relationship, although it might not say anything about the effectiveness of knowledge transfer in such a relationship.

Further, a strong relationship allows intimacy to develop between the two parties (Granovetter, 1973). Intimacy could be developed, for example, by two actors who have been in contact for a long period of time and who trust each other. Moreover, the degree of intimacy varies in different relationships. "The intimacy of co-workers' friendships can range from just working together, through acquaintanceship and friendship, to close friendship" (Haythornthwaite \& Wellman, 1998, p.1103). Thus, a friendship indicates a stronger tie than an acquaintanceship or a work relationship, and co-workers who interact closely without being close friends would have medium strong ties (Haythornthwaite, 1999).

In the past, SNA literature with a focus on the knowledge management context has often referred to the importance of strong and/or weak ties rather than considering other levels of tie strength, such as intermediate ties (e.g. Hansen, 1999; Granovetter, 1982; Levin \& Cross, 2004). In addition, the distinction between weak and strong ties varies according to the context (Marsden \& Campbell, 1984).

Nevertheless, a few studies have also considered an intermediate tie strength level in their investigations (e.g. Borrego, Osborne, Streveler, Smith \& Miller 2007; Dodds, Muhamad \& Watts 2003; Granovetter, 1974; Langlois, 1977; Marsden \& Campbell, 1984; Onnela et al, 2007; Petróczi et al., 2007). These SNA studies, mostly undertaken at the individual level, have often defined intermediate ties by the medium values of their scales used for data collection. For example, on a closeness scale 
ranging from 'not close' to 'extremely close', these medium values have been 'fairly close' or 'casual'.

In particular, Petróczi et al. (2007) focussed on the development of a continuous tie strength scale. To distinguish between weak, medium and strong ties, they used cluster analysis (A. Petróczi, personal communication, March 21, 2009), which has been applied to various contexts, for example, the geophysical field of research (Weatherill \& Burton, 2009), knowledge discovery for the semantic web (Mladenić, Grobelnik, Fortuna \& Grćar, 2009), biological research (Chen \& Wang, 2009), and marketing (Chaturvedi, Carroll, Green \& Rotondo, 1997).

This study focussed on investigating interactions and knowledge transfer in social networks that were based on work relationships in an R\&D network. These work relationships were formal or informal in nature and could be identified by specific topic-based communication in the network. Nevertheless, any two actors who were in a work relationship could have also been acquaintances or even friends, which might have influenced the strength of their relationships. However, this study focussed on measuring tie strength by the frequency of contacts (e.g. weekly, monthly, trimonthly) among people from different groups of organisations. Due to the interorganisational context, the number of people participating from each organisation also played an important role in terms of tie strength at this level. Moreover, the type of knowledge transferred may have also influenced the amount of time spent per contact and therefore the associated tie strength.

\section{Weak tie theory}

Social network theory provides the key concept of weak tie theory which was applied to the whole network approach of this study. Granovetter's (1973) weak tie theory argues that weak ties are in certain circumstances more important than strong ties. Weak ties are defined as being maintained infrequently, without emotional intensity 
or mutual confidence and having a low reciprocity of transferring information. Weak ties tend to be based on passive and opportunistic means of communication.

An example might be meeting by chance at an event such as a conference. If the opportunity to attend the conference is not present, the tie might be broken. However, weak ties need to be maintained as they might bring new information to a group (Granovetter, 1973), because they are able to give an actor access to resources outside their own immediate circle (Haythornthwaite, 1999).

The study underlying Granovetter's weak tie theory categorises strong and weak ties by their contact frequency. This categorisation is based on useful relationships that support the task of job hunting. It indicates that strong ties have contacts of a weekly frequency, whereas weak ties have a yearly contact frequency. Granovetter (1973) discovered that weak ties were much more effective than strong ties when an actor is looking for a new job.

\section{Centrality}

Centrality is a key concept in SNA (Valente et al., 2008; Freeman, 1979). The notion of 'central positions' is related to the degree of power that recipient organisations hold within a network. However, "how social structure and power are exactly related remains a topic of active research and debate" (Hanneman, 2005). From a SNA perspective, power is a "consequence of patterns of relations" among network members (Hanneman, 2005). For example, those who are situated in the central positions in a network are often the most powerful and could thus dominate the others in their interactions within the network. Having such a favoured position means that an organisation may receive "better bargains in exchanges or have a greater influence" (Hanneman, 2005). These organisations have a controlling or coordinating position that has the capacity to broker contacts among other organisations, to require 'service charges' or to isolate organisations and prevent contacts (Hanneman, 2005). 
In the context of this study, most central and therefore powerful organisations might be able to either withhold important knowledge or facilitate its transfer to other organisations in the network. This can be of significant importance when for example decisions about joint projects are made. Organisations in the most central positions may also have the influence to make decisions on ICT-based inter-organisational knowledge transfer and learning initiatives in the network.

SNA uses centrality measurements to identify these powerful positions (e.g. organisations) in a network. This important concept in SNA comprises a mixture of mathematical approaches to describe and measure centrality. A variety of centrality measures exist in social network analysis. The most frequently used centrality measures are degree, closeness, betweenness, and eigenvector (Valente et al., 2008).

Degree centrality counts how many other nodes are in direct contact with a particular node. The more nodes directly connecting to a node, the higher its degree, and therefore, the greater potential for communication and knowledge transfer.

Closeness centrality measures the distance of an actor from all others in the network (Hanneman, 2005). Borgatti (2005) explains that closeness can be seen as an index of the amount of time one expects something (e.g. knowledge) to take to flow through a network.

Betweenness centrality is a measure of the number of times an actor occurs on a shortest path between any two other actors in a network. Betweenness centrality is associated with control of information flows (Freeman, 1979). Flow betweenness (Freeman, Borgatti \& White, 1991), which is a variant of betweenness centrality, appears particularly suitable to investigate centrality in knowledge networks, as it considers various communication paths in a network (Borgatti, 1995). In addition, Polites and Watson (2008) employed information centrality to measure centrality structures in citation networks. 
Degree, closeness and betweenness centralities were proposed by Freeman (1979), whereas eigenvector centrality was proposed by Bonacich (1972). The eigenvector approach aims to identify the most central actors with the smallest distance from others in terms of the overall structure of the network. The eigenvector centrality measurement is based on factor analysis. The location of each actor in regard to its distance is called an 'eigenvalue', and the collection of such values is called the 'eigenvector' (Hanneman, 2005). The eigenvector relies on the idea that for example node1 in a network has a low centrality, but it is connected to node 2 which has a high centrality. Node1 then has a high eigenvector centrality, as it can benefit from node2's influential position (Borgatti, 2005).

Besides these four well-known centrality measures, Valente et al. (2008) also suggest information centrality as an additional measurement option. Information centrality is particularly appropriate for measuring the ability of individuals to communicate within a network (Stephenson \& Zelen, 1989). Network members with a higher information centrality are able to control the flow of information to some extent within the network. Moreover, according to Polites and Watson (2008), information centrality is considered the most appropriate measure for citation networks, as citations do not always follow the shortest path.

It is strongly recommended to match a context-specific type of network flow to the appropriate centrality measurement (Borgatti, 2005). "It is noted that the most commonly used centrality measures are not appropriate for most of the flows we are routinely interested in" (Borgatti, 2005, p.55). Different types of centrality measurements make implicit assumptions about the way in which things flow in a network (Borgatti, 2005). This study took these considerations into account.

The following sections describe the data collection and analysis procedures undertaken in the three sequential phases for each case. This starts with a description of Phase 1. 


\subsection{Phase 1: Qualitative data collection and analysis procedures}

This section describes the methods used for data collection and analysis in Phase 1.

\section{Data collection}

In all three cases, data collection began by conducting initial participant observation at a workshop. This workshop provided information about a specific environmental $R \& D$ topic to the public. Case 1 started with participant observation at a hydrology workshop. Case 2 began with observing participants in a marine biology workshop. Case 3 started with participant observation at a coastal hazards workshop. These workshops were held by a key organisation participating in this study. People from other NZ organisations working on similar topics participated. The hydrology workshop (Case 1) comprised seven workshop participants, the marine biology workshop (Case 2) had twelve participants, and eighteen participants attended the coastal hazards workshop (Case 3). Each workshop was led by at least one experienced trainer. Workshop 3, in particular, was facilitated by three trainers from three different groups of organisations: a CRI, central government and a private consulting organisation.

Participant observation is described as an observational method in which participants are aware of the observer who is allowed to interact with participants (Ridenour \& Newman, 2008). However, the actual interaction with participants was kept to a minimum in this study in order to not disturb the trainers' workshop schedule. This kind of observation also reflected the practice of 'complete observation' whereby the researcher did not participate actively (Creswell, 2003). During these initial observations, the researcher took notes on individual comments by participants on inter-organisational knowledge transfer in their profession. This also included aspects of individual and group behaviour during the workshop (Creswell, 2003). In addition, the researcher's aim was to identify key organisations in each of the three specific professional fields. 
In addition to the participant observation, the first case (the pilot case hydrology workshop) included one-on-one semi-structured interviews using eighteen openended questions. Five out of seven workshop participants and the trainer agreed to be interviewed within two weeks of the workshop. The trainer was interviewed face-toface, whereas participant interviews were conducted by phone. Interviews were recorded and transcribed. Participants had the opportunity to verify their transcribed interviews. Together with the observation notes, the interviews helped identify a number of key organisations involved in formal and informal knowledge transfer related to hydrology topics. The interviews also aimed to elicit knowledge transfer processes as well as ICT that was used to support these interactions. The interview transcripts were analysed, and results were used to develop an online survey for data collection in Phase 2, which is described in Section 3.7 .

In summary, Table 8 depicts the professional field for each case and the qualitative data collection methods used within Phase 1.

Table 8: Summary of methods used in Phase 1

\begin{tabular}{|l|l|l|}
\hline Case & Professional field & Data collection method(s) \\
\hline Case 1 & Hydrology & $\begin{array}{l}\text { Participant observation, } \\
\text { semi-structured interviews }\end{array}$ \\
\hline Case 2 & Marine biology & Participant observation \\
\hline Case 3 & Coastal hazards & Participant observation \\
\hline
\end{tabular}

Data analysis

There are some important aspects that must be considered when analysing case study research data. For example, data gathered through interviews need to be regarded as interpretations of the interviewees' understandings, and not as facts (Walsham, 1995). Thus, the interviewees' personal beliefs and experiences related to the key themes in the interview questions were considered when collecting and analysing the data. Also, the researcher's interpretation of interview data was taken into account (Miles \& Huberman, 1994). In order to achieve credible results from the case research studies,

\footnotetext{
${ }^{4}$ See from page 102
} 
careful planning was undertaken which helped to reduce the potential lack of rigour (Yin, 2003).

For example, interview guidelines were prepared for this study and revised several times. For data analysis, the widely accepted qualitative data analysis tool NVIVO was employed. In addition, it was essential to apply a widely accepted method of analysis, such as the three guidelines for qualitative data analysis provided by Miles and Huberman (1994). These guidelines include the three processes of data reduction, data display, and conclusion drawing and verification. The following figure illustrates these three inter-linked components.

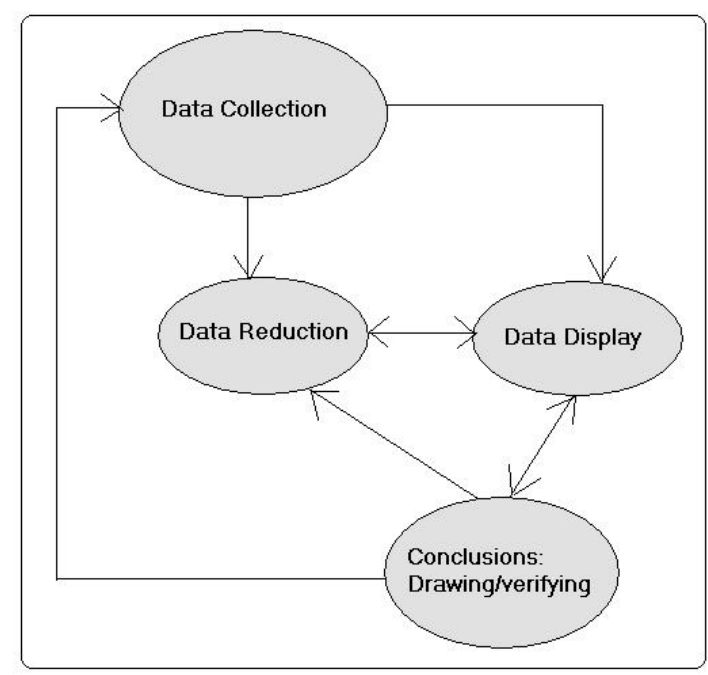

Figure 7: Components of data analysis: interactive model (Huberman \& Miles, 1998, p.181)

Data reduction allowed the researcher to simplify, abstract and transform any gathered data in order to improve the management and categorisation of the data. It can also be described as "a form of analysis that sharpens, sorts, focuses, discards, and organises data in such a way that 'final' conclusions can be drawn and verified" (Miles \& Huberman, 1994, p.11). This data reduction included coding and summarising of data, identifying key themes and writing memos. However, the data reduction process began prior to the actual data collection, as the research questions and study design were repeatedly refined and therefore influenced the categorisation of data. 
Coding is a particularly useful tool for data reduction (Paré, 2004). Paré suggests three approaches to create a coding scheme in order to provide a template for organising the data. On the one hand, the researcher can use previously defined codes, based on prior research. On the other hand, new codes can be developed "after some initial exploration of data has taken place, using an immersion or editing organising style" (Crabtree \& Miller, 2000 cited in Paré, 2004, p.249). However, Paré also suggests a more typical and intermediate approach that was applied in this study. The applied coding approach combined key themes from the literature and new codes that were added during the analysis process. The coding was supported by the use of the software programme NVIVO. The coding results can be found Table 9 .

Data display presents data in an easily accessible way. In case research analysis, interview transcriptions and documentation appear very complex and cumbersome to work with. Methods of data display can include matrices, graphs, charts and networks (Miles \& Huberman, 1994). Table 9 depicts coded categories that were developed in Phase 1 and were used for the subsequent phase of data collection (Case 2, online survey). Coded main categories and items in Table 9 were mostly expected results. They were derived from the literature review and confirmed by the interview results. However, the communication processes, initial contact and follow-up (maintenance) of the initial contact, were rather unexpected results and directly derived from the interview results. 
Table 9: Key themes as a result of data analysis in Phase 1

\begin{tabular}{|l|l|}
\hline Coded main categories & Coded items per main category \\
\hline $\begin{array}{l}\text { Advice giving/seeking as main category for } \\
\text { inter-organisational knowledge transfer }\end{array}$ & $\begin{array}{l}\text { Contact frequency for advice giving to peers } \\
\text { and advice seeking from peers at the } \\
\text { workplace }\end{array}$ \\
\hline $\begin{array}{l}\text { Contact frequency for giving/seeking } \\
\text { advice during the last year }\end{array}$ & $\begin{array}{l}\text { At least once every week, at least once } \\
\text { every month, at least once every three } \\
\text { months, at least every six months, never }\end{array}$ \\
\hline Communication processes & $\begin{array}{l}\text { Start of communication, follow-up of initial } \\
\text { communication }\end{array}$ \\
\hline Types of knowledge & $\begin{array}{l}\text { Electronic availability, meta-knowledge } \\
\text { (references, contact details), degree of } \\
\text { formality (formal/informal) and factual or } \\
\text { procedural knowledge }\end{array}$ \\
\hline Media characteristics & $\begin{array}{l}\text { Quick transfer of use, ease of use, secure } \\
\text { communication (data transfer), ability to edit } \\
\text { information before transfer, ability to save } \\
\text { information for later reuse, ability to use } \\
\text { multiple ways of communicating with text, } \\
\text { voice and/or pictures }\end{array}$ \\
\hline $\begin{array}{l}\text { Preferred ICT use for knowledge transfer } \\
\text { and informal learning after face-to-face } \\
\text { workshops }\end{array}$ & $\begin{array}{l}\text { Email, phone, video-conferencing, online } \\
\text { discussion forum, social software (wiki, blog) }\end{array}$ \\
\hline $\begin{array}{l}\text { Preferred learning methods after face-to- } \\
\text { face workshops }\end{array}$ & $\begin{array}{l}\text { Online learning, blended learning, e- } \\
\text { mentoring/coaching, interaction with experts, } \\
\text { learning community, discussion forum and } \\
\text { chat, e-book }\end{array}$ \\
\hline
\end{tabular}

The first two main categories, advice giving/seeking and the respective contact frequency, are widely used concepts in SNA studies (e.g. Cross \& Borgatti, 2001; Krackhardt \& Hanson, 1993; McEvily \& Zaheer, 1999). Communication processes were related to the development of new social contacts in the network. The goal was to identify any differences in media use between starting communication and maintaining social contact. Types of knowledge and media characteristics are also two well known key themes in the literature (e.g. Dennis et al. 2008; Nonaka \& Takeuchi, 1995; Zander \& Kogut, 1995). Preferred ICT use for knowledge transfer and preferred learning methods for informal learning after face-to-face workshops were added as two main categories. They supported one of this study's aims to recommend appropriate ICT for inter-organisational knowledge transfer and informal learning after formal face-to-face workshops that were held by this study's sponsoring organisation. 
Conclusion drawing and verification is the final stage of case research study analysis as outlined in Figure 7. Competent research remains open until the conclusions become explicit and verified. In Phase 1, interview transcripts were verified by participants. These main categories were the results of Case 1, which was similar to a pilot study. For Cases 2 and 3, these categories were adapted. This will be described in Section 3.7. In addition, Phase 3 of data collection (Section 3.8) allowed the researcher to explain and confirm key results.

\subsection{Phase 2: Quantitative data collection for conducting SNA}

This section describes the methods and tools used for data collection and analysis in Phase 2.

\section{Data collection}

SNA research can be undertaken as a qualitative study, a quantitative study, or as a mixed methods study. As this thesis aimed to collect quantitative data (specific contact frequencies) for measuring tie strength and the directions of knowledge transfer, Phase 2 followed the quantitative approach and an online survey was developed for data collection. The survey is a commonly used method to collect data in SNA studies (Carrington, Scott \& Wasserman, 2005). The SurveyMonkey tool ${ }^{5}$ was used to develop the online survey. A copy of this online survey can be found in Appendix 1.

The structure of the online survey was based on the interview results of data collection in Phase 1 of Case 1. Statements gathered in a qualitative data collection phase can be used as specific items and themes to create a survey "that is grounded in the views of the participants" (Creswell, 2003, p.221). Such a sequential approach has

\footnotetext{
${ }^{5} \mathrm{http}: / /$ www.surveymonkey.com
} 
been previously applied by Jonkeren and Huysman (2006) who identified informal knowledge networks within one organisation. Jonkeren and Huysman (2006) interviewed participants to identify knowledge domains, then used a follow-up questionnaire to collect data about knowledge transfer in these identified domains.

This thesis also employed such a follow-up survey to collect data about interorganisational knowledge transfer in the professional domains of hydrology, marine biology and coastal hazards. Due to the overall goal of exploring inter-organisational knowledge transfer, the survey was of an explorative nature. One main goal of this phase was to collect quantitative data for measuring and visualising tie strength and the directions of knowledge transfer within the three social networks. Potential survey participants were contacted by email. Two groups of people were considered potential survey participants for each case. Firstly, the workshop participants and trainers were invited to take part in the survey. Secondly, members of professional societies in New Zealand that were strongly related to one of the topic-based cases, (hydrology, marine biology and coastal hazards) were invited. The survey questions included the frequency of contacts, the direction of knowledge transfer, the type of knowledge transferred and media used for knowledge transfer. Contact frequency was divided into once every week, once every month, once every three months, once every six months and never. The directions of knowledge transfer were giving advice to peers and seeking advice from peers. The types of knowledge transferred and the media used for transferring it are described later in this section. Additional measurement of quantitative data comprised descriptive and procedure based analysis methods to identify patterns of inter-organisational knowledge transfer and the most powerful groups of organisation in the R\&D networks.

The design of the online survey varied slightly between the survey used in Case 1 and the survey employed in the subsequent two cases (Cases 2 and 3), as Case 1 was initially planned as a pilot study. However, participation numbers were higher than expected and most results were deemed appropriate for comparison and useful for further analysis (cross-case analysis). In Case 1, sixteen questions were posed in the 
survey. They were categorised as (A) personal information (demographic data), (B) communication processes (media use for start/maintenance), (C) giving advice (frequency, type of knowledge and media characteristics), (D) seeking advice (frequency, type of knowledge and media characteristics) and (E) ICT and additional learning services (e.g. learning community, online learning, interaction with experts or discussion forums and chat) to current workshops/seminars/conferences.

In terms of types of knowledge provided in the survey sections (C) and (D), knowledge was differentiated into electronically accessible and electronically inaccessible knowledge. This differentiation was necessary as the aim of the research was to identify ICT that supports knowledge transfer, and knowledge that was not electronically accessible could be considered as part of the practical implications. This distinction was previously made by Maier et al. (2005). Apart from the electronic availability, knowledge was differentiated into meta-knowledge (contact details and references), formal knowledge (facts or how-to) and informal knowledge (facts or how-to). Meta-knowledge and the difference between factual knowledge and how-to (procedural) knowledge have a relatively long tradition in knowledge management research (e.g. Connell 1995; Zander \& Kogut 1995), whereas the differentiation between formal and informal knowledge is a more recent development (Maier et al., 2005). No research has been conducted to investigate how these various types of knowledge are used by diverse social relationships.

This initial survey design was improved and adapted after Case 1. A number of adaptations were made, comprising conceptual changes to support the goals of this study and structural changes to enhance user-friendliness. The conceptual improvements included an enhancement of section (B) Communication processes, which asked study participants for the types of media used when starting or maintaining a social contact at the workplace. This section was refined as study participants described another communication scenario besides starting and maintaining a social contact: the initial follow-up after starting a new social contact. Therefore, after Case 1, communication processes were differentiated into firstly 
starting a new contact, secondly the initial follow-up after the first contact, and thirdly the (long-term) maintenance of a social contact.

A second conceptual improvement related to the options of preferred learning methods that were used for the survey in Case 1. The aims were to reduce the initial number of options to increase user-friendliness and also streamline the initial options with a focus on ICT and Wenger and Ferguson's (2006) knowledge services framework. Firstly, the initial seven options were reduced to four options: online learning, online interaction with experts, online discussion forums and chat, and ebooks. These options for preferred learning methods were strongly related to Wenger and Ferguson's (2006) framework for knowledge services which was a theoretical underpinning of this study. Each of the four options represented one ICT-based knowledge service that belonged to one of the four possible main categories of the framework: exploring, guiding, participating, or informing. Online learning represented the informing knowledge service, online interaction with experts represented the guiding knowledge service, online discussion forum and chat referred to the participating group of knowledge services, and finally the e-book option referred to the exploring group of knowledge services in the framework. Due to their specific characteristics (exploring, guiding, participating, informing), each service represents one specific knowledge service option for study participants, who could only choose among four distinct options. The knowledge ecology model with its various knowledge services has been described in Section 2.5.3. This conceptual improvement helped streamline options of preferred learning methods in relation to the theoretical underpinning used for this study.

A third conceptual change was undertaken by including a new question about learning method characteristics in order to relate the preferred learning methods (e.g. interaction with experts, online learning) to preferred learning method characteristics such as participating, guiding and informing. This helped to evaluate the views of study participants in relation to Wenger and Ferguson's (2006) knowledge services framework. 
A fourth, and final, significant conceptual enhancement was added to the two questions about the type of knowledge used when either seeking advice from peers or giving advice to peers in the workplace. The type of knowledge added was the tacit dimension of knowledge (Nonaka \& Takeuchi, 1995). Tacit knowledge was briefly explained to study participants as 'knowledge that people carry in their minds, but which is not written down (as codified knowledge is)'. Electronically unavailable knowledge was then differentiated into explicit (codified) knowledge and tacit (noncodified) knowledge.

Overall, a few questions relating to learning characteristics and ICT-based knowledge transfer and learning were placed earlier in the survey to emphasise their importance and make sure most study participants would answer them. Some minor rephrasing was included. One rephrasing related to the media characteristic 'provides secure information (data transfer)'. This was replaced by 'provides a trustworthy environment', which seemed to be more appropriate for the inter-organisational knowledge transfer scenario. The main questions about the frequency of contact study participants had with people working in other organisations remained the same throughout the whole study.

In all cases, a selection of diverse media were provided as answer options to the communication process questions under category (B) and (E) when asking about ICT options for learning after a face-to-face workshop. These ICT options were email, phone, face-to-face meetings (work meetings, workshops, seminars, and conferences), video-conferencing, online discussion forums, and wikis or blogs. This presents a combination of media previously used for similar research (Haythornthwaite, 1999) and current social software.

The study participants' trust in the ability and integrity of the researcher is essential to the data gathering process. Thus, it was important to establish a good relationship with (potential) participants before the collection of data. This was carried out 
through interaction with social network members during the initial observation period and, more especially, during the follow-up interviews as part of data collection Phase 1 of Case 1 . In order to enhance study participants' understanding of this research and to stress confidentiality a brief information sheet was given to potential participants prior to participation. This information sheet is attached in Appendix 2.

In order to maximise the number of responses, current e-research literature suggests a variety of supporting features such as progress indicators, missing data messages and reassurance of confidentiality (Best \& Krueger, 2004). Therefore, in this online survey, the researcher provided a progress indicator and included missing data messages when obligatory questions were not answered.

\section{Data analysis}

Analysis of social network data collected from the online survey was carried out by using one of a range of software tools developed specifically for SNA. Some of the most commonly used software packages are Ucinet, Agna, Krackplot, Netdraw, Fatcat and InFlow. These programs are based on graph theory and produce mathematically correct network analysis (Lumsden, Breathnach \& Richards, 2003). A decision to employ the software package Ucinet (Borgatti, Everett \& Freeman, 2002), which was currently available in version 6.188 , was made, as this program offers comprehensive relational data analysis opportunities, specifically the tie strength and centrality measures required by this study. Ucinet (version 6.188) also includes Netdraw (version 2.080) which is a visualisation tool for analysed data. The quantitative data were analysed and illustrated as a network map of knowledge transfer across each social network. Descriptive statistics helped to analyse patterns of knowledge transfer in the networks. SNA results were illustrated by using directed and weighted graphs, as tie strength and the directions of knowledge transfer were measured. This represents the highest level of data that can be used in network analysis (Lumsden et al., 2003). 
Key concepts of SNA such as tie strength, direction of flow (knowledge transfer) and centrality measures were employed when analysing survey data for each case in Phase 2. Preferred media characteristics and the different types of knowledge transferred within different types of relationships (strong or weak) were analysed. Furthermore, preferred ICT for knowledge transfer and learning was analysed. Details about the implications for ICT support can be found in Chapter 8 .

\subsection{Phase 3: Qualitative data collection and analysis using a Wiki tool}

This section describes the data collection and analysis undertaken in Phase 3 of the sequential three-phase mixed methods approach of this study.

\section{Data collection}

Data collection in Case 1 was initially planned without conducting a Phase 3. Despite its initial pilot study characteristics, a higher than expected participation number and also valuable results for further analysis could be achieved and it was thus decided to treat Case 1 as a fully-fledged case, rather than only as a pilot study. However, during Case 1 the need for further clarification and confirmation of key survey results became apparent. Thus, Phase 3 was then added for all three cases.

In Phase 3 of each case, workshop participants were invited by email to participate in answering open-ended questions to explain and confirm a number of results of Phase 2 (the online survey). Questions focussed on five main areas. The first area related to the use of social software (e.g. wikis and blogs) and social network tools by study participants for work-related topics. The second area covered the survey participants' preferred media characteristics as indicated in the survey results. The third area referred to the preferred knowledge services for learning. The fourth area included questions about the types of relationships between diverse organisations in the respective professional field of either hydrology, marine biology or coastal hazards. 
The final goal was to elicit examples of the types of knowledge transferred in social relationships, and to identify whether there were any resulting differences for weak and strong relationships.

The follow-up questions were provided online to study participants by a Wiki tool (editable web page). This wiki for data collection allowed selected users to jointly edit HTML documents (Anderson \& Kanuka, 2003). In particular, users were allowed to edit, delete, and add to the page containing the answer section to the follow-up questions. In other words, the wiki provided a space for collectively answering the follow-up questions. The major difference between an online discussion group and a wiki is this communal creation of web pages (Anderson \& Kanuka, 2003). This results in a series of continuously changing document versions that are archived per change and user instead of a long discussion list. Research methods that could have been applied instead are a focus group or interviews. The ideal result of these answer pages would have been documents with a collaborative discussion of answers from study participants. However, this would have asked for a higher level of commitment which was beyond the goals of this study. This study applied the wiki in order to achieve answers that confirmed survey results and provided examples as further explanations.

Three 'feedback wikis', one for each of the three main areas, were configured and employed for each case. Screenshots of the wiki tool can be found in Appendix 3. The Wiki tool was part of an OpenSource interactive e-learning program called Moodle ${ }^{6}$. Moodle is an internationally established Open Source program for providing e-learning resources. Moodle follows a constructivist learning approach that provides the user with a number of interactive features (Forment, 2007). For example, the tool provides the opportunity to set up online courses as well as online communities including social software tools such as wikis and blogs.

\footnotetext{
${ }^{6} \mathrm{http}: / /$ elearningpilot.umoodle.co.nz/
} 
In the literature, the Wiki tool is described as an e-research method for data collection (Anderson \& Kanuka, 2003). E-research describes Internet tools for data collection and analysis using both qualitative and quantitative approaches (Anderson \& Kanuka, 2003). Best and Krueger (2004) recommend a number of supportive features for eresearch. These features include providing instructions (explaining instrument access and usage), personalising contacts in invitations and sending at least two reminders. Two pages of instructions with screenshots on how to log on and use the feedback wikis for responding to the follow-up questions were sent as a pdf document attachment along with the email invitation. Wherever possible, potential participants were addressed personally. From the researcher's point of view this aspect was significant for achieving responses. For each case and wiki system, at least two reminders were sent to potential participants.

Apart from the advantages of confirming and explaining survey results, using the Wiki tool as an electronic data collection method helped overcome time and location differences between the researcher and study participants. Participants could choose to take part whenever it was convenient for them. The researcher did not need to travel to visit study participants or phone them for interviews. Unlike interviews with participants, there was no need to transcribe the data, as it was readily available in the wikis. Moreover, study participants could have been inspired by viewing other participants' answers before answering the questions themselves. On the downside, this aspect might have influenced study participants to add similar answers to already existing ones. However, study participants who agreed with other participants' answers only needed to confirm their agreement without repeating the same answer. In summary, the use of wikis for obtaining study participants' feedback was considered an appropriate approach for data collection in Phase 3. The following sections expand on the data analysis conducted. 
For data analysis, answers were checked against the goals of confirming and explaining the survey results in the four main areas considered: (1) the use of social software and networking tools in the workplace; (2) results of preferred media characteristics and knowledge services; (3) the types of relationships between organisations; (4) as well as the type of knowledge transferred in the social relationships. Analysis of the data in Phase 3 allowed for some data triangulation which helped verify results and strengthened aspects of data reliability and validity.

In particular, the three guidelines for qualitative data analysis as provided by Miles and Huberman (1994) were employed in this phase as in Phase 1. The guidelines include data reduction, data display and conclusion drawing and verification. Data reduction included coding and summarising of data in regard to the five key themes as outlined. An extract of the coding results is displayed in the following table. 
Table 10: Coded examples of Phase 3 explaining/confirming key themes

\begin{tabular}{|c|c|}
\hline Coded main categories & Coded items per main category \\
\hline $\begin{array}{l}\text { The use of social software in the } \\
\text { workplace }\end{array}$ & $\begin{array}{l}\text { Restricted quality of information; missing knowledge } \\
\text { on how to use social software; easier and quicker to } \\
\text { talk face-to-face when possible; social software is } \\
\text { unfamiliar to scientists (due to generation); health } \\
\text { issues when working at the computer; missing } \\
\text { organisational resources to provide social software } \\
\text { for use at an inter-organisational level }\end{array}$ \\
\hline $\begin{array}{l}\text { Preferred media and learning method } \\
\text { (knowledge service) characteristics }\end{array}$ & $\begin{array}{l}\text { Ability to save information for later reuse allows the } \\
\text { researchers to revisit/reuse information. Trustworthy } \\
\text { information is also important; "the most accessible } \\
\text { sources are the ones that have the greatest utility"; } \\
\text { references are useful, particularly for beginners; } \\
\text { later reuse of information for modelling purposes; } \\
\text { quick transfer and ease of use are also important. } \\
\text { The ability to save information for later reuse is } \\
\text { convenient. }\end{array}$ \\
\hline Preferred online learning features & $\begin{array}{l}\text { Clear images, well-designed database/search } \\
\text { engine; hot links, online quizzes; discussion forums } \\
\text { for general information about new research; online } \\
\text { bibliographic database; free research papers in pdf } \\
\text { format; online seminars/lectures with the option of } \\
\text { emailing/skyping with the presenter afterwards as } \\
\text { part of discussion; better use of online groups.; e- } \\
\text { newsletters; option to pose general questions on } \\
\text { methodology and trends. Preferences for push } \\
\text { services that automatically deliver information; } \\
\text { professional societies' web pages could be a starting } \\
\text { point for providing online learning services. }\end{array}$ \\
\hline $\begin{array}{l}\text { The types of relationships between } \\
\text { organisations }\end{array}$ & $\begin{array}{l}\text { Connections are there but communication could be } \\
\text { improved; joint projects; formal agreements and } \\
\text { contracts; a lot of informal relationships based on } \\
\text { shared interests/expertise (writing joint research } \\
\text { papers) or former colleagues; sometimes } \\
\text { competition among organisations due to the science } \\
\text { funding model in NZ; informal research assistance in } \\
\text { the form of providing general and/or local knowledge } \\
\text { or a co-ordinator. Informal relationships can turn into } \\
\text { formal relationships. }\end{array}$ \\
\hline The type of knowledge transferred & $\begin{array}{l}\text { Research papers in pdf format; reports, journal } \\
\text { papers; verbal advice; published material such as } \\
\text { conference papers; unpublished data material; data } \\
\text { archives; issues with transferring large amounts of } \\
\text { data; images; personal observations and opinions; } \\
\text { experiences and suggestions; technological/ } \\
\text { instrumental knowledge. }\end{array}$ \\
\hline
\end{tabular}

This final phase of data collection and analysis, Phase 3, helped explain and confirm the results of Phase 2 for each case. Therefore, results and conclusions became 
explicit and verified in Phase 3. This conclusion drawing and verification finalised the analysis in the three cases of this multiple case research study.

The next section outlines the procedures carried out as part of the final cross-case analysis.

\subsection{The cross-case analysis}

The process underlying the cross-case analysis in this study was a systematic comparison of all three cases. This process is typically suitable for the identification of patterns and relationships among different cases (Miles \& Huberman, 1994). The overall idea behind this study's cross-case analysis was to search for common patterns in order to answer the research question: "What are the knowledge transfer structures and processes among organisations within an R\&D network ?". Therefore, it was necessary to go beyond the initial impressions using structured and diverse perspectives on the data that resulted from each individual case analysis (Eisenhardt, 1989).

According to Eisenhardt (1989), three cross-case analysis tactics are recommended: 1) select categories and look for within-group similarities coupled with inter-group differences, 2) select pairs of cases and list the similarities and differences between each pair, and 3) divide the data by source to identify results from different types of data collection. The cross-case analysis techniques employed in this study followed the first and second tactics in order to identify common patterns. An additional division of the data by source was not considered suitable, as the data collection methods in this study were employed consecutively and therefore covered different areas.

In addition, this study's cross-case analysis employed three techniques to display data and allow patterns to be identified as suggested by Miles and Huberman (1994): 
(1) Firstly, key categories that resulted from each individual case analysis were compared. Resulting key categories of the cross-case analysis are shown in Table 11.

Table 11: Key categories/findings of the cross-case analysis

\begin{tabular}{|l|}
\hline Structural properties of the networks for each case \\
\hline The types of knowledge transferred for advice giving and seeking activities \\
\hline Media/ICT related results \\
\hline
\end{tabular}

A detailed description of these results can be found in Chapters 4-6.

Then a meta-matrix summarised these results and facilitated pattern recognition across the individual cases by looking for common characteristics. Identified commonalities include structural properties, such as the network topologies, centrality aspects and levels of interaction. In addition, the cross-case analysis identified a new category that was not revealed in any of the case descriptions: intermediate ties were identified as important ties that connect key players for inter-organisational knowledge transfer.

Further, cross-case analysis findings revealed similarities among the cases in regard to the more influential groups of organisations in the networks, typical types of knowledge transferred, ICT used and study participants' preferred media characteristics as well as knowledge services for learning. A copy of this resulting meta-matrix which includes the summarised categories can be found in Appendix 4. Details about these results are described in the cross-case analysis results in Chapter 7.

(2) A clustered summary table was developed to organise the identified categories in an efficient manner, guided by the research objectives. This table summarises and structures the key categories identified in the meta-matrices. An example of a clustered summary table is given in Appendix 5. 
In particular, the six resulting key categories are:

1. Ontological network structure

2. The importance of intermediate ties

3. Key players in the networks

4. Types of knowledge transferred in the networks

5. Media/ICT use and preferred media characteristics in the networks

6. Preferred ICT-based knowledge services in the networks

Chapter 7 presents the results in these categories in detail.

(3) In addition, matrix diagrams helped display key categories along two dimensions. For example, key players in all three cases could be illustrated by using diagrams that show the groups of organisations per case in relation to their levels of interaction. Thus, groups of organisations with a high level of initiating and/or receiving relationships could be revealed. These diagrams are illustrated in Section 7.3. Having an illustrative purpose, the diagrams are comparable to the scatter plots suggested by Miles and Huberman (1994). In regard to inter-organisational knowledge transfer, the diagrams assisted in identifying and illustrating groups of organisations as being either key initiators or key receivers of relationships for knowledge transfer.

Overall, these cross-case analysis techniques proved useful, as they enabled the comparison of different cases from diverse perspectives. Most of the key categories were identified by the individual case analysis write-ups conducted prior to this crosscase analysis. More importantly, by conducting the cross-case analysis, the significance of intermediate ties has been identified as an additional key category. Chapter 7 will expand on these results.

The following section presents some delimitations in regard to this thesis' goals. 


\subsection{Delimitations}

This study aimed to explore specific knowledge transfer structures and processes in three inter-organisational social networks within a larger R\&D network in the environmental sector in New Zealand. Thus, the location for data collection was limited to New Zealand. In addition, as three social networks were explored by multiple case studies, this thesis was limited to a group of participating individuals from diverse organisations. However, due to the applied SNA approach, the findings appear transferable to other R\&D networks, which include similar organisations, in New Zealand and other OECD countries.

Due to the extended scope of the study and the focus on several key concepts of SNA, the study needed to focus on a certain domain in the given context and therefore discard other concepts. In particular, the study focussed on the identification of interorganisational knowledge transfer structures and processes, but it did not aim to develop an instrument for measuring the effectiveness or success of this knowledge transfer. Instead, this study aimed to give advice to the organisations to select appropriate ICT for their inter-organisational knowledge transfer and learning. Moreover, the identification of specific knowledge transfer structures and processes provided solid background data to identify collaborative technology support for the three social networks studied. This study aimed to identify ICT to support knowledge transfer, but the research was not investigating IT adoption or technology acceptance. Implications about the use of appropriate ICT to support inter-organisational knowledge transfer and learning will be drawn in Chapter 8 of this thesis.

\subsection{Summary of the research strategy, design and methods}

This study employed a sequential mixed methods research design. This included case research as the leading research strategy comprising a multiple case study with three consecutive cases. The research process for each case started with a qualitative phase (Phase 1), followed by a quantitative phase (Phase 2) and a third and final qualitative 
phase (Phase 3) as illustrated in Figure 8. As Phases 1 and 3 were both qualitative in nature, the qualitative approach was dominant in this study. This research design was guided by the research questions to identify and analyse inter-organisational knowledge transfer structures and processes, underlying social relationship characteristics and the use of supportive ICT.

Phase 1 included participant observations for all three cases and interviews with participants which were conducted after the first workshop (Case 1). Firstly, notes from observations were used to refine the interview guidelines. Results of the analysed interviews helped develop an online survey to collect data for a subsequent quantitative SNA in Phase 2. The survey was personalised for each case.

Finally, a third phase of qualitative data collection and analysis helped confirm and explain various survey results regarding study participants' use of social software, their preferred media characteristics, and types of knowledge transferred when giving advice to and seeking advice from peers with whom they have social relationships in the workplace. Results from all three phases helped to answer the first two research sub-questions which then provided solid background information for answering the third research sub-question. This research design is illustrated in Figure 8.

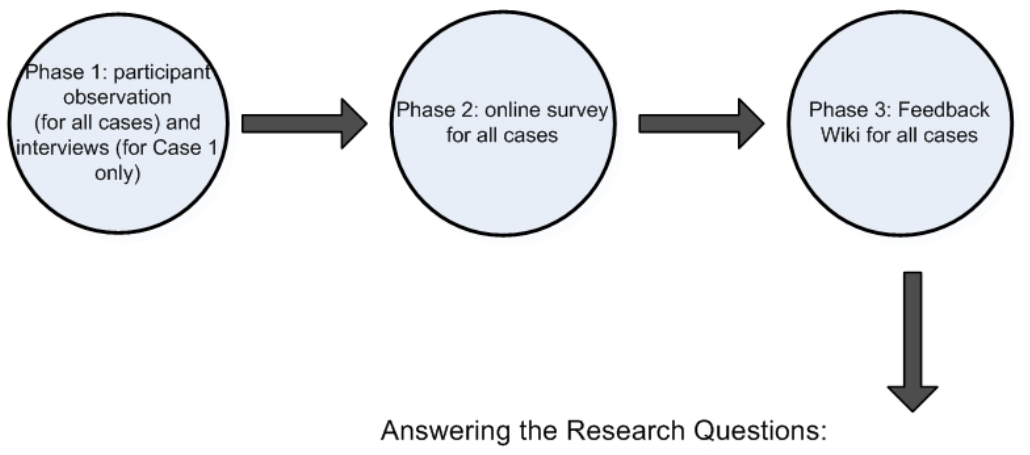

- What are the key structural properties of advice networks and their implications for knowledge transfer?

- Which types of knowledge are transferred in the advice networks?

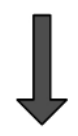

- How can ICT support these knowledge transfer structures and processes?

Figure 8: Research phases 
Due to its mixed methods approach, the research project required well organised data collection and analysis procedures, including well-structured interview guidelines and questionnaire.

This chapter has outlined and justified this study's case research strategy and the sequential mixed methods design. It has described the data gathering procedures and analysis methods and tools including SNA as the key methods and techniques to investigate inter-organisational social relationships and the associated knowledge transfer. The unit of analysis has been defined as the social relationship at the interorganisational level. Figure 9 illustrates the three different social networks which comprised the multiple cases of this study. Case 1 was akin to a pilot study which helped ensure the quality of the research design. The research undertaken in Case 1 provided valuable insights and experiences that could be integrated into the following two cases and the three-phase research process design. Data collection and analysis for two other social network cases, Cases 2 and 3, followed Case 1. Despite its initial pilot study characteristics, a higher than expected participation number and also valuable results for further analysis (cross-case analysis) could be achieved and it was thus decided to treat it as a fully-fledged case, Case 1, rather than only as a pilot study. Each case was analysed separately, and a cross-case analysis was conducted in the end. The final cross-case analysis revealed similar patterns of inter-organisational knowledge transfer structures among the three social networks.

An overview of the organisation of the data collection and analysis per case as well as the cross-case analysis is illustrated in the following figure. 


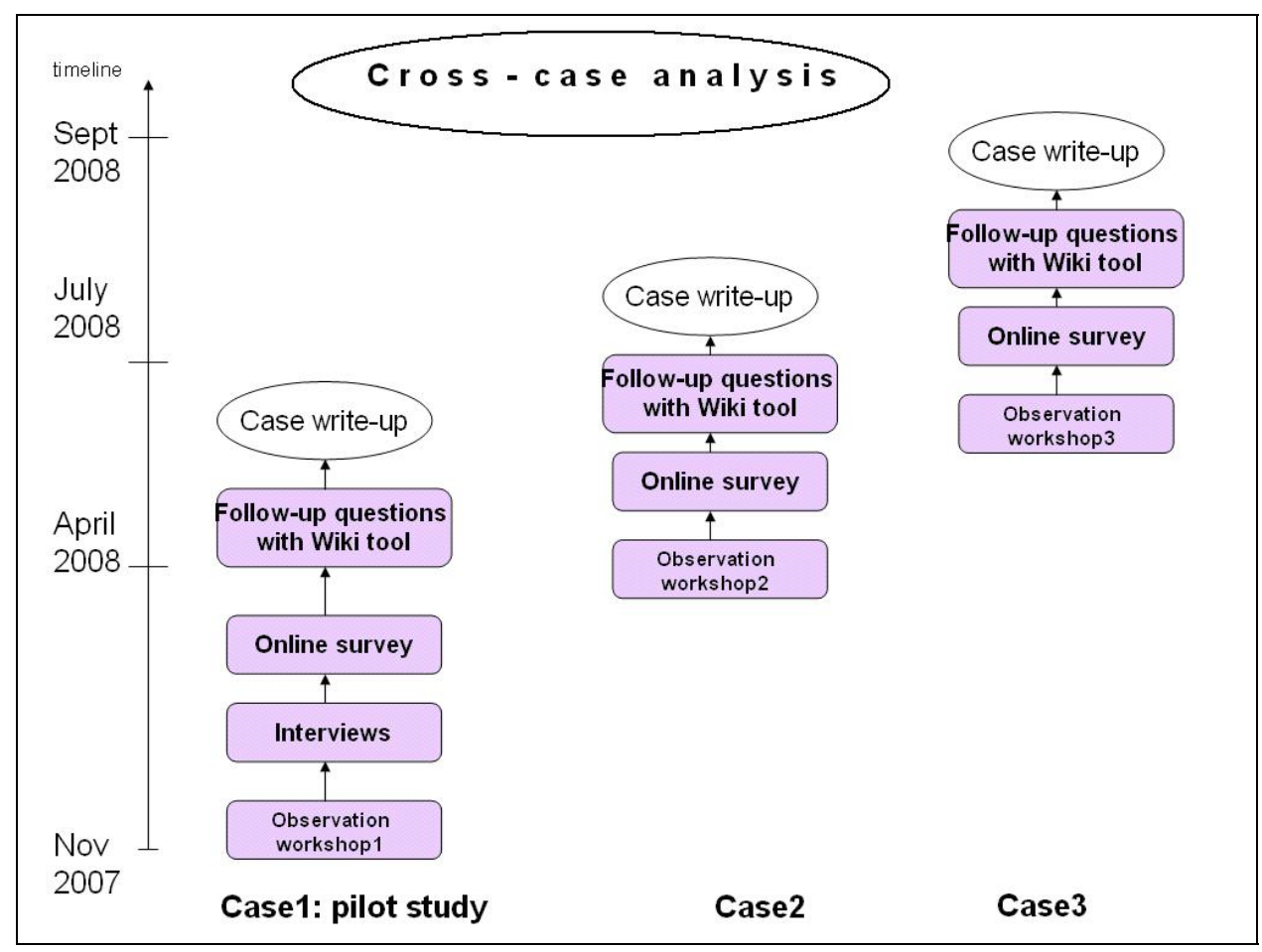

Figure 9: Research process outline

Figure 9 also shows the sequential data collection for the cases starting in November 2007. As Case 1 had pilot study characteristics, more time was taken in beginning ( 6 months) to tease out the research process and develop lessons learned and integrate improvements into the design of the following cases.

Moreover, the results from all cases were compared to establish key structures that may be transferable to social networks in other contexts. Quality assurance was addressed by employing the qualitative concepts of trustworthiness as well as reliability and validity guidelines for the quantitative SNA part of the study.

The following chapters will demonstrate and describe this study's key results regarding inter-organisational social relationships and the associated knowledge transfer through advice giving and seeking activities in the environmental R\&D sector in New Zealand. This will start with a description of Case 1 results. 


\section{Case 1 results}

This chapter firstly describes the study participants' characteristics in Case 1 . Then, a description of study participants' types of knowledge transferred in their social relationships is given. Next, key findings in regard to structural properties of this social network are described. This includes the direction of knowledge transfer and tie strength. In addition, centrality measures are conducted to identify the most influential organisations in the network. Finally, this chapter concludes with a summary and discussion of the key findings.

\subsection{Study participant characteristics}

This section describes the characteristics of study participants who took part in the online survey. In Case 1, 80 participants from 27 organisations responded. The organisations in which the participants were employed were active in the professional field of hydrology in New Zealand. The hydrology topic was taken as a boundary for a social network including people who were working at diverse organisations, but shared the same work interests and therefore communicated with each other in some form (e.g. by giving advice to peers or seeking advice from peers).

During the data collection, five key organisations that were at least partly active in the same professional field (hydrology) were identified. Some key organisations were more specialised in hydrology topics than others. Individual study participants from these five key organisations had various roles in the field of hydrology ranging from environmental officer to water resource scientist. However, the most common job titles were analyst, scientist, engineer, and a slightly smaller number of consultants.

The 27 organisations included five key organisations: CRI 1, Regional Council 1, CRI 2, Regional Council 2, and a private business organisation. The remaining 22 organisations were mostly private businesses, but also included CRIs and local 
government (regional/district/city councils). The research process helped identify five key groups of organisation, which are outlined in Table 12.

Table 12: Characteristics of key groups of organisations in Case 1

\begin{tabular}{|l|l|l|}
\hline Key groups & Ownership & Core business activities \\
\hline CRIs & Public & Basic research, consulting, publications \\
\hline Local government & Public & $\begin{array}{l}\text { Applied research, consulting, } \\
\text { publications }\end{array}$ \\
\hline Universities & Public & $\begin{array}{l}\text { Basic research, applied research, } \\
\text { publications }\end{array}$ \\
\hline Private businesses & Private & $\begin{array}{l}\text { Applied research, consulting, } \\
\text { publications }\end{array}$ \\
\hline Central government & Public & $\begin{array}{l}\text { Consulting and monitoring, reporting and } \\
\text { publications }\end{array}$ \\
\hline
\end{tabular}

Overall, a group of organisations could be defined by ownership (public, private) and its core business activities (e.g. basic research, applied research, consulting, reporting and publications). Workshops and seminars that were offered to the public were held by only one CRI.

Bearing in mind the information given about membership numbers by the professional societies contacted for data collection, the overall response rate was calculated at around 42 percent. This is a reasonable result and comparable to response rates (e.g. 9\%; 29\%;30\%;34\%) in other studies that investigated interorganisational knowledge transfer (e.g. Cummings, 2001; Priestley, 2003) or intraorganisational knowledge transfer with SNA (e.g. Chan \& Liebowitz, 2006). However, due to a pre-selection of certain (groups of) organisations for data collection and limited control over the actual organisational affiliation of participants, this response rate remains an approximate figure.

Inter-organisational knowledge transfer between these groups of organisations happened in either formal business relationships, in publicly offered seminars, workshops and conferences, or in social relationships. In addition, an established network for knowledge transfer between Regional Councils ${ }^{7}$ was identified.

\footnotetext{
${ }^{7}$ http://groups.localgovt.co.nz/
} 
In terms of contact frequency, inter-organisational knowledge transfer happened with a three to 6-monthly frequency, whereas intra-organisational knowledge transfer always showed a higher frequency. Seeking and giving advice were equally important within one organisation and also in an inter-organisational context.

Not all 80 participants answered each question. However, a majority of 54 participants answered the two survey questions on contact frequencies. These were of particular interest to the SNA part of this chapter. Participants tended to opt out of the survey more often in the second than in the first part. This may lead to the conclusion that they considered the survey was too long. However, no particular non-respondent bias could be determined.

The following table illustrates the number of study participants, who answered the two survey questions on the frequencies of advice giving and seeking activities, from each of the five organisational key groups. These numbers were of particular interest for the SNA undertaken in this chapter.

Table 13: No of study participants per organisational key type in Case 1

\begin{tabular}{|l|c|}
\hline \multicolumn{1}{|c|}{ Group of organisation } & No of study participants \\
\hline Private businesses & 22 \\
\hline CRIs & 15 \\
\hline Local government & 14 \\
\hline Universities & 2 \\
\hline Central government & 1 \\
\hline All organisations & 54 \\
\hline
\end{tabular}

Compared to the number of participants from private businesses, CRIs and local government, there was a low number of study participants from universities and central government. This may reflect the selection of key groups of organisation for the SNA data collection which did not include universities or central government in Case 1 . In addition, there were only a few individuals from central government who were members of the professional society that was contacted for survey distribution in Case 1. 
Overall, a higher number of men $(83.8 \%)$ than women $(16.3 \%)$ participated in the survey. Supporting data can be found in Appendix 6. Overall, there were more males who were new at their job than females as indicated in Figure 10.

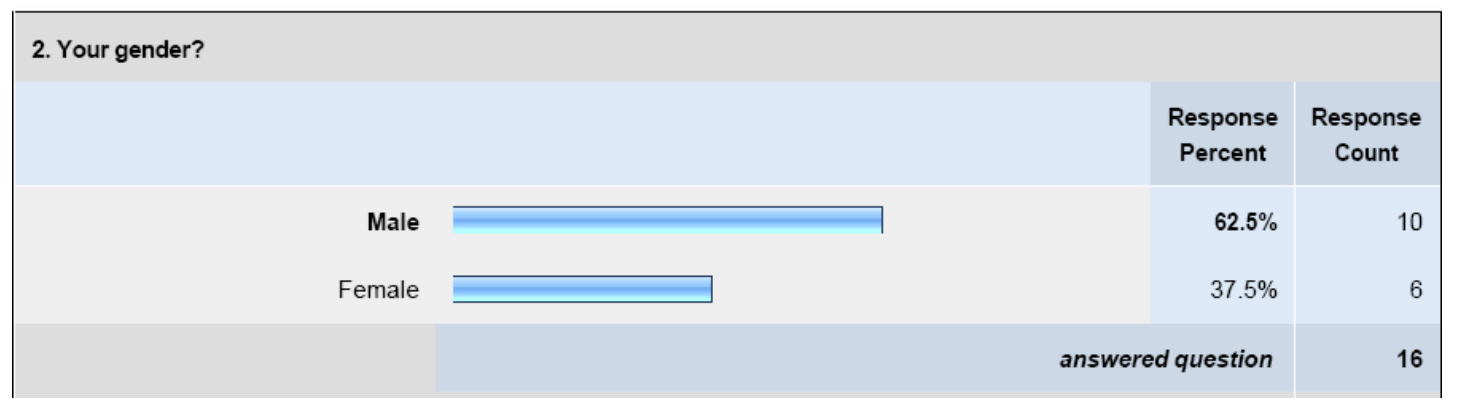

Figure 10: Job beginners (0-3 years) by gender in Case 1

The following figure shows the job experience of study participants. A relatively high number of experienced experts (11-30 years in their job) participated. This question on job experience was answered by 54 out of 80 participants.

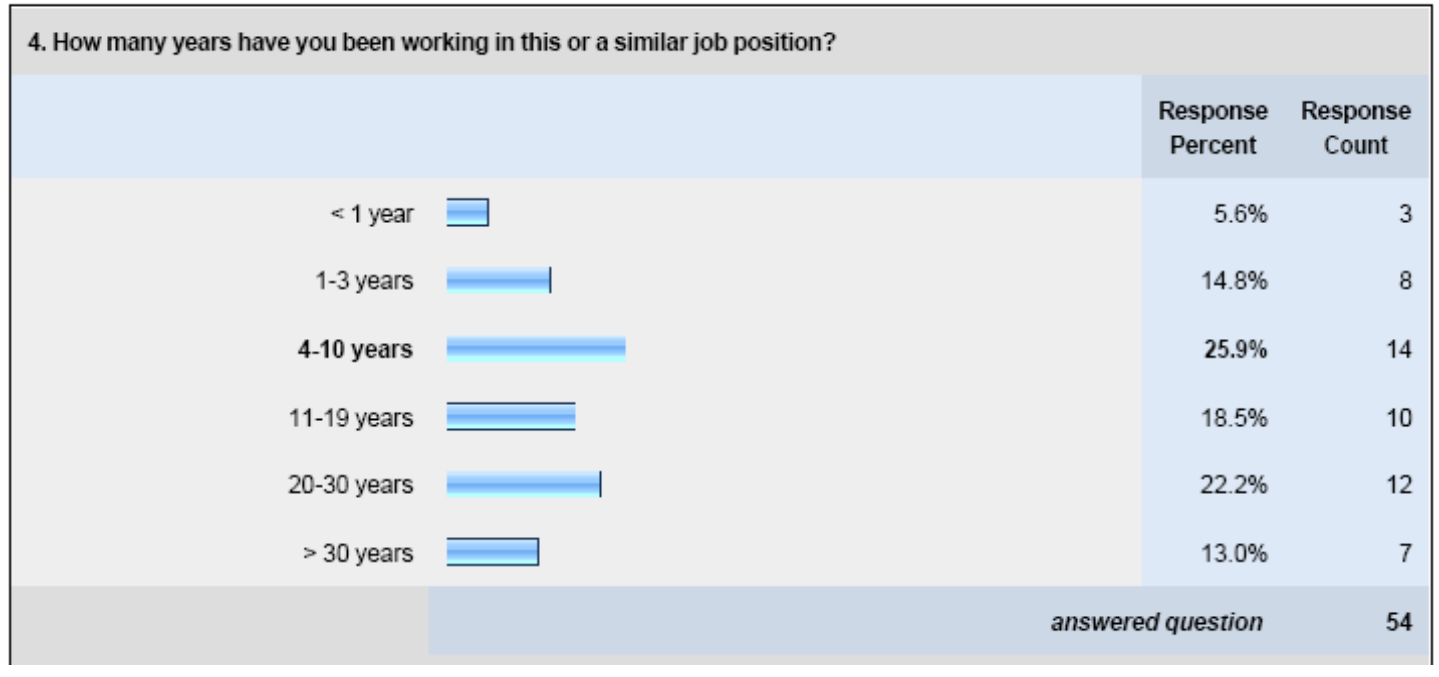

Figure 11: Study participants by job experience in Case 1

The following paragraphs introduce the possible relationships between the study participants described. 


\section{Relationships among study participants}

As all organisations were engaged in the same professional field (hydrology), one would expect a number of formal business relationships such as alliances as well as various informal relationships, for example through friendships or acquaintanceships. Current literature on inter-organisational knowledge transfer also confirms the existence and importance of informal relationships (e.g. Easterby-Smith et al., 2008; Perez-Nordtvedt et al., 2008; Sammarra \& Biggiero, 2008). In the context of this study, the co-existence of formal and informal relationships has been confirmed by interviewees and also study participants in the follow-up questions to the online survey (Phase 3). Examples are:

"There are joint projects, formal agreements and contracts, but a lot of relationships are also quite informal based on friendships (former colleagues etc.), traditional habits and expertise in the same or a related work field" (Study participant 1),

“There are some projects that require formal interactions" (Study participant 2),

or ".... joint projects, contracts, but also friendship and expertise in the same field (the latter mostly has to do with writing papers)" (Study participant 3).

There were also some critical comments from study participants such as "Connection between organisations is there, but links and communication could be improved".

From a business perspective, relationships between the participating organisations could be characterised as formal relationships in the form of customer relationships, supplier relationships, and partner relationships (e.g. joint projects, alliances etc).

Case 1 comprised mostly partner relationships, as participating key organisations were engaged in the same field of hydrological science and research. However, certain specific relationships in terms of knowledge transfer could be identified 
during the data collection. For example, Regional Council 1 was a supplier of new knowledge (rules, guidelines and procedures) for the small private business organisation that transferred this knowledge again to its clients. CRI 1 was the largest organisation with the most experienced scientists and researchers in the field. For example, regional councils were interested in drawing on this wealth of experience. CRI 1 was therefore mostly a supplier of knowledge to the public. Regional councils were knowledge seekers in this case.

Further, relationships between the participating organisations could be characterised by the groups of organisations that belong to the relationships, for example a publicprivate relationship or a public - public relationship. However, in a network a certain number of relationships is possible, which in mathematical terms is the result of $\mathrm{Nx}(\mathrm{N}-1) / 2$, whereas $\mathrm{N}$ represents the number of actors in a network. In this study, the number of actors was represented by the number of groups of organisations. Thus, Case 1 revealed the following possible undirected relationships between the five participating key groups of organisation:

Table 14: Possible relationships between groups of organisations

\begin{tabular}{|c|c|}
\hline $\begin{array}{ll}> & \text { CRIs - local government } \\
> & \text { CRIs - private businesses } \\
> & \text { CRIs - central government } \\
> & \text { CRIs - universities }\end{array}$ & $\begin{array}{l}\text { Central government - local government } \\
>\quad \text { Central government - private businesses }\end{array}$ \\
\hline $\begin{array}{ll}> & \text { Universities }- \text { CRIs } \\
> & \text { Universities - local government } \\
> & \text { Universities }- \text { private businesses } \\
> & \text { Universities }- \text { central government }\end{array}$ & $>$ Private businesses - local government \\
\hline
\end{tabular}

Table 14 shows ten possible inter-organisational relationships. Moreover, when considering the diverse contact frequencies (once every week, once every month, once every three months, once every six months, or never) and the direction of knowledge transfer, the variety of relationships multiplies. The following section describes how the direction of knowledge transfer was considered for the SNA part of this study. 
In their social relationships, study participants transferred diverse types of knowledge that were another significant factor influencing knowledge transfer in interorganisational advice networks.

\subsection{Knowledge characteristics}

This section outlines the types of knowledge focussed on in this study. In addition, the importance of various types of knowledge to different types of social relationships (e.g. weak/strong) is explained.

In Case 1, knowledge was differentiated into electronically accessible and electronically inaccessible knowledge. This differentiation was important, as the aim of the research was to identify ICT that supports knowledge transfer, and knowledge that was not electronically accessible could be prioritised for practical implications. This distinction has previously been made by Maier et al. (2005). Apart from the electronic availability, knowledge was differentiated into meta-knowledge (contact details or references), formal knowledge (facts or how-to) and informal knowledge (facts or how-to).

Meta-knowledge and the difference between factual knowledge and how-to (procedural) knowledge have a relatively long tradition in knowledge management research (e.g. Connell 1995; Zander \& Kogut 1995), whereas the differentiation between formal and informal knowledge is more recent (Maier et al., 2005). The following list summarises these types: 
$>$ electronically accessible knowledge

$>$ electronically inaccessible (but codified) knowledge

$>$ meta-knowledge

$>$ formal knowledge

$>$ informal knowledge

$>$ factual knowledge

how-to knowledge

Electronically accessible knowledge can be electronic documents, data archives or contributions to discussion forums. In contrast, electronically inaccessible (but codified) knowledge refers to books and paper-based documents. All of the following types of knowledge can either be electronically available or unavailable. Metaknowledge is knowledge about knowledge in general, which means, for example, knowledge about other sources of knowledge or contact details of experts. Formal knowledge describes knowledge that needs to follow a certain formal presentation, whereas informal knowledge refers to conversation and word-of-mouth knowledge. Informal knowledge is typically transferred in communities such as social networks. Factual knowledge describes data-based information, for example data archives. In contrast to factual knowledge, how-to-knowledge refers to procedural and other experience-based types of knowledge.

Specific types of knowledge were transferred in diverse relationships with different tie strength. The types of knowledge that were more important than others to study participants when transferring knowledge are shown in Table 15 and were categorised as: formal knowledge (factual and electronically accessible), informal knowledge (factual and how-to knowledge, both electronically inaccessible) and meta-knowledge (partly electronically accessible).

Table 15 summarises the survey results for giving and seeking advice in terms of types of knowledge transferred. Survey participants stated the following types of knowledge were significant for their inter-organisational knowledge transfer. This 
transfer of knowledge is differentiated into weak and strong relationships (ties). The concept of tie strength that explains weak and strong ties is explained later in this section $^{8}$.

Table 15: Types of knowledge transferred compared between giving advice and seeking advice network in Case 1

\begin{tabular}{|l|c|c|c|c|c|}
\hline & $\begin{array}{l}\text { Electronically } \\
\text { accessible formal } \\
\text { documents } \\
\text { (facts) }\end{array}$ & $\begin{array}{l}\text { Electronically } \\
\text { accessible } \\
\text { references to } \\
\text { other sources of } \\
\text { knowledge }\end{array}$ & $\begin{array}{l}\text { Electronically } \\
\text { inaccessible } \\
\text { informal how-to } \\
\text { knowledge }\end{array}$ & $\begin{array}{l}\text { Electronically } \\
\text { inaccessible } \\
\text { informal } \\
\text { factual } \\
\text { knowledge }\end{array}$ & $\begin{array}{l}\text { Electronically } \\
\text { inaccessible: } \\
\text { experts' contact } \\
\text { details }\end{array}$ \\
\hline $\begin{array}{l}\text { Giving strong } \\
\text { ties }\end{array}$ & $\mathbf{X}$ & $\mathbf{X}$ & $\mathbf{X}$ & & \\
\hline $\begin{array}{l}\text { Giving weak } \\
\text { ties }\end{array}$ & & $\mathbf{X}$ & $\mathbf{X}$ & & \\
\hline $\begin{array}{l}\text { Seeking } \\
\text { strong ties }\end{array}$ & & $\mathbf{X}$ & & $\mathbf{X}$ \\
\hline $\begin{array}{l}\text { Seeking weak } \\
\text { ties }\end{array}$ & $\mathbf{X}$ & & & $\mathbf{X}$ & \\
\hline
\end{tabular}

The results in the table indicate that three types of knowledge were not electronically accessible for a large number of participants: informal how-to knowledge, informal factual knowledge and experts' contact details.

Study participants in strong relationships mostly transferred different types of knowledge compared to participants in weaker relationships. For advice-giving activities, study participants in strong relationships transferred mostly formal factual knowledge and references to other sources of knowledge. Both of them were electronically available. They also gave advice in the form of informal how-to knowledge (electronically inaccessible). This kind of knowledge could have involved predictions on floods and rainfall intensity as well as simple opinions, as reported by a study participant in Phase 3 of data collection.

In comparison to strong relationships, study participants in weaker relationships were not interested in transferring formal documents. Published conference articles or data archives were examples of transferred formal documents provided by study participants in Phase 3. When seeking advice, participants in strong relationships transferred predominantly references to other sources of knowledge (electronically

${ }^{8}$ Section 4.3 .2 
available). They also received informal how-to knowledge and experts' contact details, both of them not electronically available.

In comparison to strong relationships, study participants in weaker relationships were interested in formal documents (electronically accessible) and informal factual knowledge (electronically inaccessible). In contrast to the forms of knowledge transferred when giving advice, advice-seeking activities appeared to transfer knowledge that was not as easily available electronically. In summary, three electronically inaccessible types of knowledge that were of particular interest to this study could be identified: informal how-to knowledge, meta-knowledge and informal factual knowledge.

When looking at different types of knowledge that were transferred by people with different levels of job experience, one meaningful detail was revealed. Formal knowledge was transferred (given and sought) predominantly by people with either very little (0-3 years) or very high levels of job experience ( $>20$ years). Study participants in the medium range of job experience (11-19 years), in contrast, did not transfer formal knowledge.

In relation to the group of organisation (public/private, CRI or university), it became apparent that study participants from regional councils (public) were more interested in transferring informal knowledge than participants from private businesses.

In summary, this section has described how different types of knowledge were transferred throughout this inter-organisational advice network in the professional field of hydrological science and research in Case 1. The types transferred most were electronically accessible references to other sources of knowledge, electronically inaccessible informal how-to knowledge and electronically accessible formal documents (facts). Results showed that the types of knowledge varied between strong and weak ties as well as givers and seekers of knowledge. Supporting data are attached in Appendix 7. 
The following section reports key findings related to structural properties of the advice network in which the described key (groups of) organisations participated.

\subsection{Key findings related to structural properties of the advice network}

Key findings report on the direction of knowledge transfer, as well as tie strength. All results refer to the key groups of organisation identified for this case. Appropriate centrality measures were selected to analyse more central groups of organisation for knowledge transfer in the social network of Case 1. Finally, descriptive statistics that describe the level of interaction in the advice network are outlined.

\subsubsection{Direction of knowledge transfer}

Advice giving to peers and advice seeking from peers in the workplace were chosen as the activities that define the direction of knowledge transfer in an interorganisational setting. A variety of previous literature used 'advice giving and seeking' as a type of knowledge transfer when looking at how people transfer knowledge in their professions (Cross et al., 2001; Haythornthwaite, 1996). This also defines the existence of a sender and a receiver for knowledge transfer. The transfer can be uni- or bi-directional between sender and receiver.

In SNA visualisations, directed data are illustrated by an arrow (showing that there is some sort of direction) and values that are shown along the lines, which demonstrate the tie strength for each direction. The direction considered in SNA refers to either the initiation of a relationship (active) or the receiving of a relationship request (passive). Therefore, the initiation could be realised by offering advice to another organisation or by approaching another organisation when seeking advice, whereby the latter interpretation has been adapted to this research. The receiving of a relationship request refers to an incoming advice offer or to a request from another 
organisation seeking advice, whereby the latter interpretation has been adapted to this research.

However, initiation is the significant part of starting a relationship. If these directions can be considered, they typically do not have the same values. For example, the CRI gave advice monthly to the private business organisation, whereas this private organisation gave advice to the CRI on a 6-monthly basis during the last year. Undirected data, in contrast, imply the same data for advice-giving activities as for advice-seeking activities.

Some measures in SNA (e.g. information centrality or multiple centrality measurements) symmetrise data for mathematical reasons. This symmetrised data represent undirected data, and therefore no direction is considered for knowledge transfer between organisations. SNA data can also be symmetrised manually by a variety of options, for example using either maximum values or average values for both directions. For example, if the CRI gave advice monthly to the private business organisation, whereas this private organisation gave advice to the CRI only on a 6monthly basis during the last year, the contact frequency per direction would either be monthly (maximum contact frequency) or an average contact frequency such as 3monthly.

These assumptions about the direction of knowledge transfer in Case 1 are also true for the following two Cases 2 and 3.

Linkages that show the direction of knowledge transfer can also be weighted by the level of tie strength. The following section explains and describes how tie strength has been measured in the inter-organisational context of Case 1. 


\subsubsection{Tie strength}

A whole network approach, as explained in Chapter 3, was used for collecting and visualising tie strength. Tie strength between organisations and/or groups of organisations was measured by contact frequency for giving and seeking advice activities (once every week, once every month, once every three months, once every six months, or never) and the number of study participants from each organisation. Supporting data are attached in Appendix 8.

For Case 1, the researcher aggregated the organisations into organisational types. For example, all study participants from regional councils were aggregated at the local government level and all study participants from CRIs were aggregated at the CRIs level. These aggregations were done when analysing the data. This level investigating diverse groups of organisations (inter-groups of organisations) was employed to provide comparable network data for a later cross-case analysis (Chapter 7) regarding network characteristics such as size (number of relationships) and types of members.

Figure 12 illustrates this advice network showing the key organisations selected prior to data collection. The figure indicates the strength and direction of knowledge transfer within the network. Tie strength is illustrated by line thickness. Direction of knowledge transfer comprised giving and seeking advice and is illustrated by an arrow at the end of the line. 


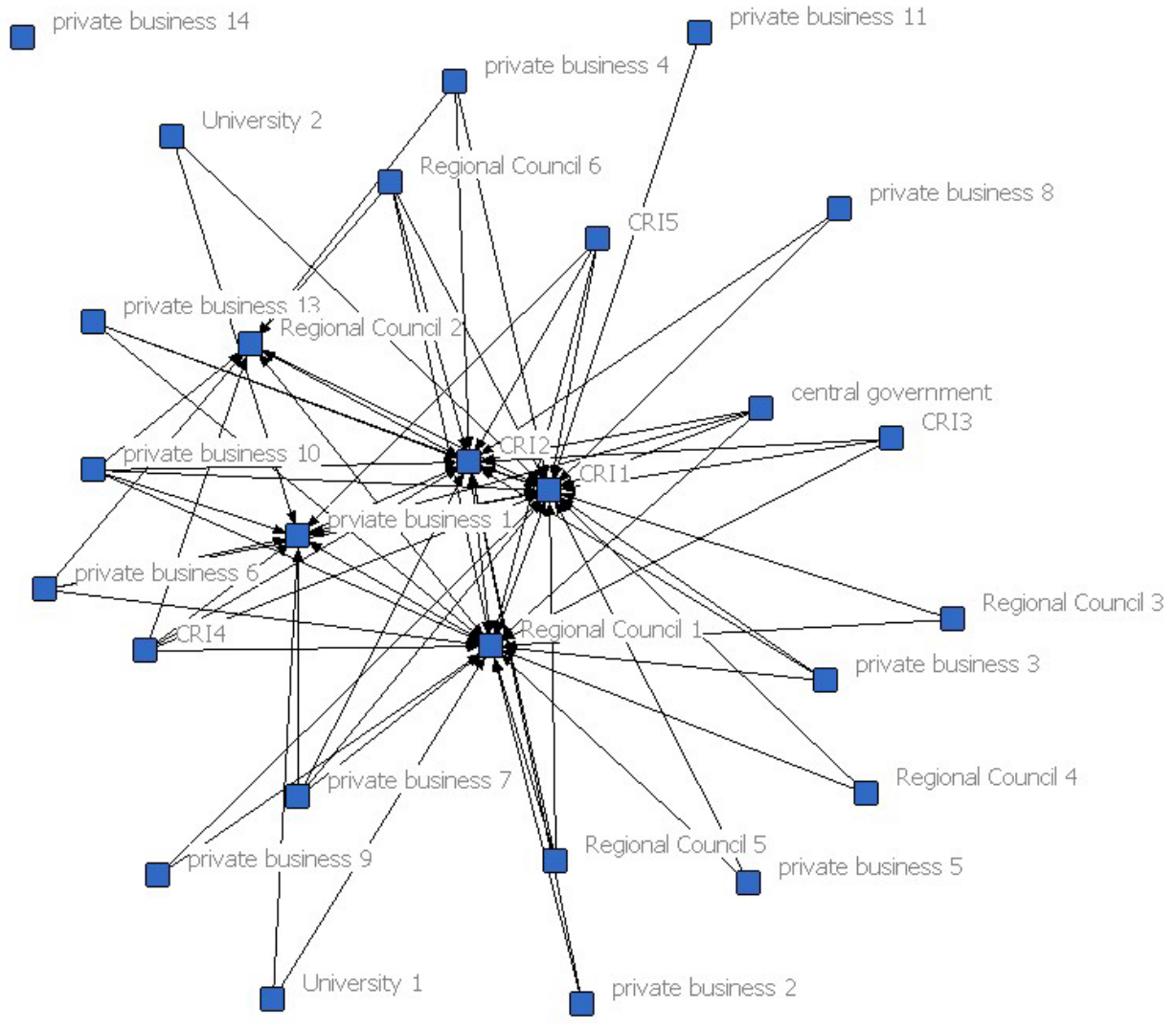

Figure 12: Advice network of all organisations in Case 1 (whole network)

Figure 12 presents the results of measuring tie strength and illustrates the whole network of 27 organisations or groups of organisations in the hydrology field that participated. They either gave advice to people from other organisations in the network or sought advice from people working in other organisations during the last year. Five key organisations with a total number of 27 study participants were strongly connected. These can be identified by the thicker lines in the network of Figure 12.

In addition, a number of weak ties (thin lines) with a total number of 22 organisations comprising 27 individual study participants remain. One isolated organisation (private business 14) that indicated intra-organisational knowledge transfer only could also be identified. Only one person participated from this organisation. Coded data underlying these tie strength measurements can be found in Appendix 9 . 
The direction of knowledge transfer (giving or seeking advice) and the contact frequency as well as the number of study participants were used for measuring tie strength. As SNA measurements can also be conducted using unweighted or undirected data, the measurement of tie strength in this study represents the highest level of data that can be used in network analysis (Lumsden et al., 2003). Undirected data imply the same data for advice-giving activities as for advice-seeking activities. Unweighted data would represent 'dichotomised' data which only consider 'contact frequency' (1) or 'no contact frequency' (0). No rating in contact frequency, such as weekly or monthly frequency, would be considered.

However, if weighted data are considered, as for this social network, values can be shown along the lines in a diagram. They demonstrate the tie strength for each direction from each organisation. However, demonstrating all values among 27 different organisations did not appear to be the best way of visualising weighted data in Figure 12. Instead, the following figure uses this visualisation method for outlining the tie strength among the five key organisations. It is a snapshot that focuses on the stronger relationships presented in the previous figure.

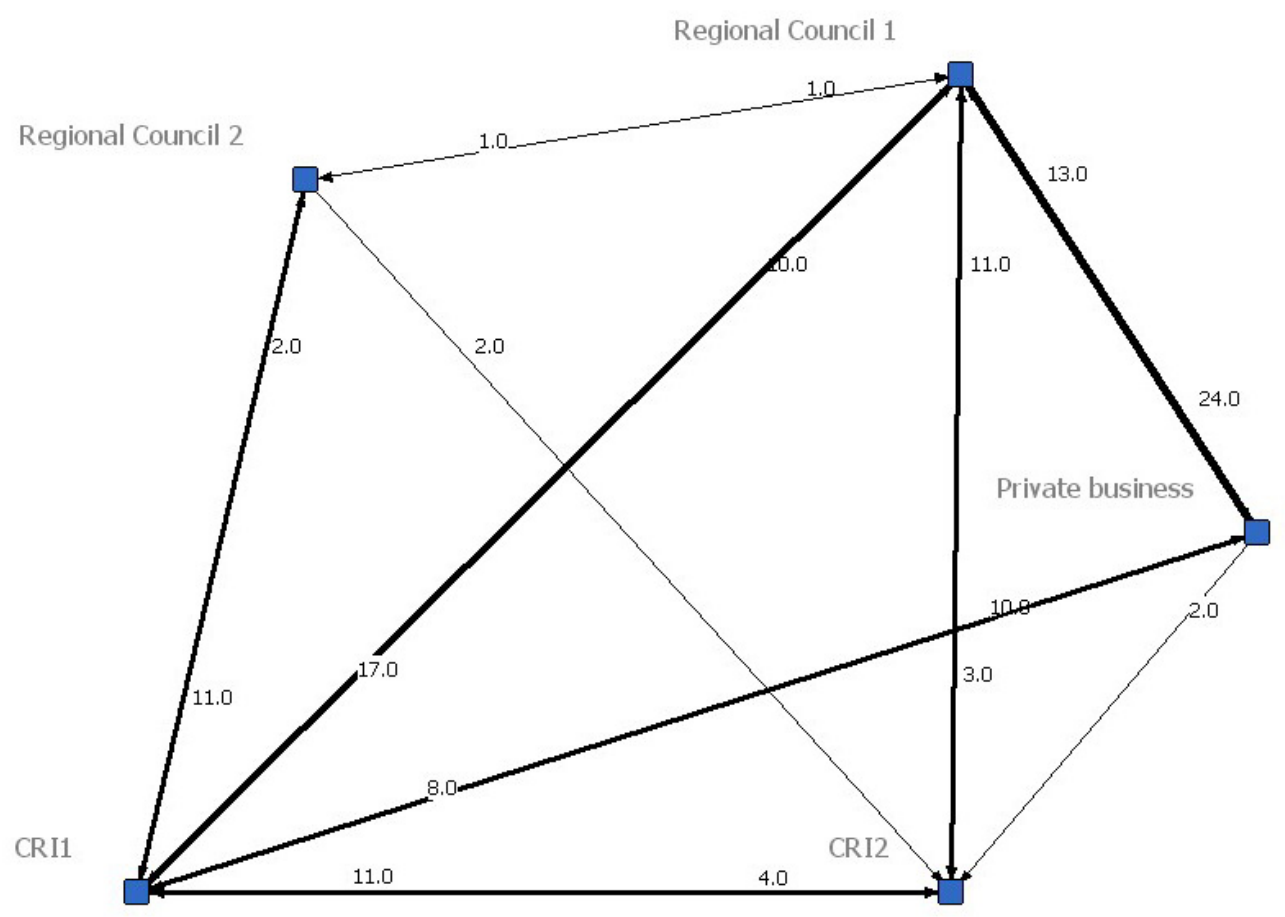

Figure 13: Weighted tie strength between key organisations 
The organisations attracting the highest number of receiving relationships can be identified by the high values associated with the direction of knowledge transfer, for example Regional Council 1 and the private business or CRI 1 and Regional Council 1. Regional Council 1 received requests for relationships (and therefore knowledge transfer) from CRI 1 (17) and the private business (24). In addition, CRI 1 also received a relatively high number of requests for knowledge transfer from the private business (13). These high numbers of relationship requests reflect the existence of a high level of domain knowledge within CRI 1, Regional Council 1 and the private business. Due to the low number of study participants from Regional Council 2 in Case 1, there was also a low number of initiating and receiving relationships for Regional Council 2.

The organisations initiating the highest number of relationships were CRI 1 and Regional Council 1. In particular, CRI 1 requested knowledge transfer at a high level from Regional Council 2 (11), CRI 2 (11) and Regional Council 1 (17). Due to its high number of initiating as well as receiving relationships, CRI 1 is a well-connected domain leader and possibly knowledge broker in the field of hydrology.

The high tie strength among CRI 1, Regional Council 1 and the private business may indicate that there was a large number of informal relationships. The strength of these relationships may be due to the fact that the respective organisations involved were located in the same regional area. Weaker relationships (lower contact frequency and/or number of study participants) were identified between Regional Council 2 and Regional Council 1 as well as CRI 2. Another weak relationship (thin line and low tie strength values in Figure 13) was revealed between the private business and CRI 2.

Besides the analysis of these key organisations, all participating organisations were aggregated at a level reflecting groups of organisations as described earlier in this chapter. Figure 14 depicts tie strength between the five groups of organisations. In particular, the figure represents study participants from CRIs, private businesses, universities, and local and central government. In comparison to Figure 13, the 
following figure, Figure 14, shows for example all regional councils and CRIs at an aggregated level. Regional councils are categorised as local government.

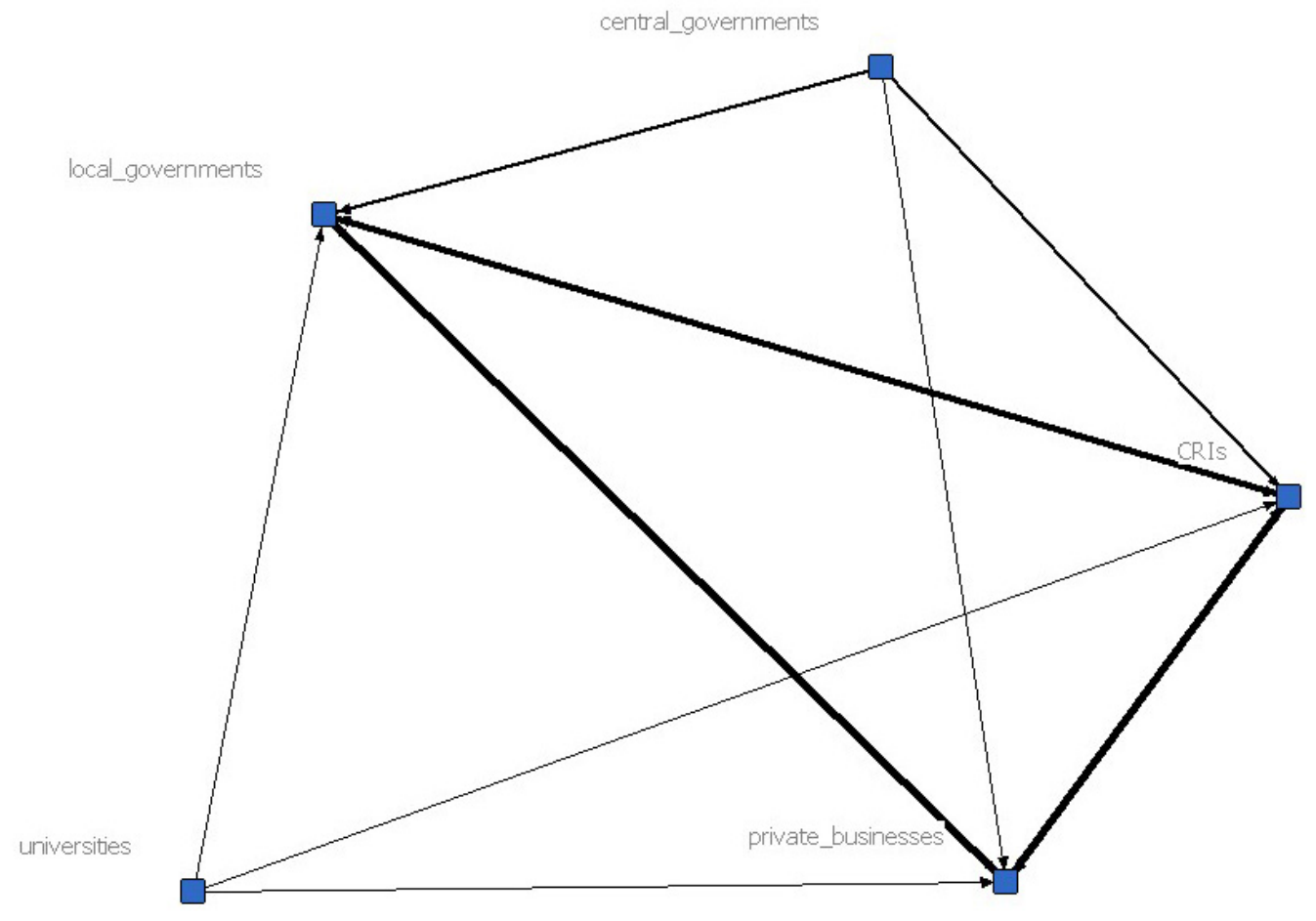

Figure 14: Tie strength between groups of organisations in Case 1

The analysis underlying Figure 14 includes 54 study participants from the five key groups of organisation: CRIs, local government, private businesses, universities and central government. The results relating to tie strength adhere strongly to the results among the key organisations as shown in Figure 13. Although data were aggregated at this stage, the tie strengths among the group of CRIs, private businesses and local government organisations are still the highest. These three key groups of organisation represent the domain leaders in the field of hydrology.

Tie strength was measured at the inter-organisational level in terms of contact frequency and number of study participants per organisation. Thus, numbers of study participants have influenced tie strength measures. In general, a lower number of study participants would have led to a lower tie strength value (with the same contact frequency). However, apart from this number of study participants, some ties between 
groups of organisations showed a higher contact frequency than others. On average, inter-organisational knowledge transfer happened on a 3- to 6-monthly basis. A monthly frequency for both giving and advice-seeking activities could be recognised by the following ties: central government and CRIs as well as central and local government. A monthly frequency for advice-seeking activities only could be recognised by the following ties: private business and local government as well as universities and CRIs.

In summary, this analysis revealed not only different levels of tie strength and directions of knowledge transfer among participating organisations, but also important results on the aggregated level representing groups of organisations in the New Zealand hydrology sector. For example, the important roles of CRIs, private businesses and local government could be highlighted.

In addition to these tie strength measurements, centrality aspects were considered in this thesis. The following section expands upon appropriate centrality measures for knowledge networks.

\subsubsection{Centrality aspects}

This section focuses on appropriate centrality measurements for knowledge networks such as the inter-organisational advice network under investigation. A description of the notion of centrality is given and related to this study's context. The most important issues to consider for undertaking centrality measurements for knowledge networks are explained. This implies a significant difference between centrality measurements most suitable for networks in which explicit electronically available or unavailable knowledge is transferred. The centrality measurements were conducted at the group-of-organisation level. Supporting data for all tables showing centrality measurement scores in this section are attached in Appendix 8. 
The notion of 'central positions' is related to the degree of power that recipient organisations hold within a network. For example, those who are situated in the central positions in a network are often the most powerful members and could thus dominate the others in their interactions within the network. Therefore, from an SNA perspective, power is a 'consequence of patterns of relations' among network members (Hanneman, 2005). Having such a central position means that an organisation may have a greater influence, for example on decisions made in the network. A central organisation may also be privileged in exchanges (Hanneman, 2005).

In the context of this study, these powerful organisations are able to either withhold important knowledge or facilitate its transfer to other organisations in the network. Therefore, these organisations have had a controlling or co-ordinating position that includes the capacity to broker contacts among other organisations, to require 'service charges' or to isolate organisations or prevent contacts (Hanneman, 2005). In particular the facilitation of knowledge transfer in the network may include decisions about the appropriate ICT support.

SNA uses centrality measurements to identify these powerful positions (e.g. organisations) in a network. This important concept in SNA comprises a mixture of mathematical approaches to describe and measure centrality. However, "how social structure and power are exactly related remains a topic of active research and debate" (Hanneman, 2005). A variety of centrality measures exist in social network analysis. The four most commonly used measures of centrality are degree, closeness, betweenness, eigenvector and information centrality which were described in Section 3.5 .

Due to this variety of different centrality measures, it is strongly recommended to match a specific type of network flow (context-specific) to the appropriate centrality measurement. Different types of centrality measurements make implicit assumptions 
about the way in which things flow in a network. "It is noted that the most commonly used centrality measures are not appropriate for most of the flows we are routinely interested in" (Borgatti, 2005, p.55), as the implicit centrality measures' assumptions do not match the network flow characteristics. According to Borgatti (2005), centrality measures differentiate between different typologies of network flows that are based on two dimensions:

$>$ firstly the kind of trajectory that traffic may follow (geodesics, trails, paths or walks) and

secondly the method of spread (broadcast, serial replication, or transfer).

This section briefly explains the kinds of trajectory that traffic may follow. A 'geodesic' is the expression used in network theory for the shortest path. A shortest path describes a direct connection between actor $\mathrm{A}$ and actor $\mathrm{B}$. In contrast to the shortest path, a 'walk' includes all possible and repeatable connections in a network (unrestricted sequences). 'Trails' and 'paths' restrict some connections: in a 'trail' no connection is repeated, whereas in a 'path' no connection is repeated and no actor is visited twice (Borgatti, 2005).

As this study asked participants for peers with whom they exchanged knowledge in the form of advice giving and seeking activities, the shortest path seems to be the predominant option for the first dimension. However, the case of transferring metaknowledge, such as contact details of experts to peers at the workplace, enhances the geodesic concept in terms of additional paths that need to be considered. Actor A may ask Actor B for the contact details of Actor $\mathrm{C}$ who is an expert in a specific field. In addition, the researcher suggests that knowledge in an advice network typically does not pass the same link twice, but it may pass the same actor multiple times (trail).

Besides the kind of trajectory, the method of spread can also be significant. Different centrality measurements include different assumptions about how knowledge can spread in a network. Three options can be considered (Borgatti, 2005): parallel 
replication (broadcast), serial replication and transfer. Knowledge exchanged by email is an example of parallel replication (Borgatti, 2005). An email can be sent to several other people at the same time, but a copy still remains in the sender's mailbox.

However, if the email is sent to only one person, the knowledge contained in an email can only be replicated once (serial replication). Besides email, other examples for replication are gossip or attitudes (Borgatti, 2005). In regard to knowledge however, the medium for its replication or how this medium is used may influence the ability for serial or parallel replication. Most Case 1 participants used media such as email and face-to-face meetings to support peer-to-peer knowledge exchange. But these media could have also supported group communication, for example group meetings or when using email distribution lists. This indicates that advice giving and seeking could take place in a one-to-one scenario (serial replication), but also in a one-tomany scenario (parallel replication) dependent on the medium and how it is used.

In contrast to a replication, transferred objects like used goods, money or a package do not remain with the sender (Borgatti, 2005). Knowledge could be transferred in the form of a book or a paper-based document. This reveals that this method of spread is dependent on the type of knowledge. Two types of knowledge have been of particular interest to this study: explicit electronically available knowledge and explicit electronically unavailable knowledge. These types are explained in relation to this study's context in the following paragraphs.

Explicit electronically available knowledge, in the form of formal knowledge and meta-knowledge, was the most important type that study participants sought or gave in the advice network. Explicit knowledge that is electronically available can be replicated or distributed simultaneously (broadcasted) dependent on the media support (e.g. email listserv). Most ICT-based media, such as email or discussion forums, allow users to keep an electronic copy of their messages or documents. 
Explicit, but electronically unavailable, knowledge was relevant but not as important as electronically available knowledge to study participants. However, in this form (e.g. as a document or book), knowledge may spread by transference. When you give a book away, you cannot keep it at the same time. However, one important thing to consider is that documents or books could be copied. Then, this type of knowledge would be replicated rather than transferred. Nevertheless, this knowledge appears to be the only type that can be transferred, as explained by Borgatti (2005).

This brief discussion leads to the researcher's conclusion that there must be different types of centrality measurements for different kinds of knowledge networks. In the context of this study, it was not possible to distinguish clearly between networks that exclusively transfer one specific type of knowledge, as study participants always selected them in combination. However, Case 1 participants mostly used explicit electronically available knowledge. Secondly, explicit but electronically unavailable knowledge was also relevant. Table 16 summarises the characteristics of the two types of knowledge starting with the most important one.

Table 16: Types of knowledge and their flow characteristics

\begin{tabular}{|l|l|l|}
\hline Type of knowledge & Kind of trajectory & Method of spread \\
\hline $\begin{array}{l}\text { Explicit electronically } \\
\text { available knowledge }\end{array}$ & $\begin{array}{l}\text { Serial or parallel replication } \\
\text { dependent on medium }\end{array}$ & Shortest path or trail \\
\hline $\begin{array}{l}\text { Explicit electronically } \\
\text { unavailable knowledge }\end{array}$ & $\begin{array}{l}\text { Transfer or replication (dependent } \\
\text { on paper-based copies) }\end{array}$ & Shortest path or trail \\
\hline
\end{tabular}

These results guided the selection of appropriate centrality measures for this context of inter-organisational knowledge transfer and learning.

The following types of centrality measures have been chosen:

- $\quad$ closeness centrality

- $\quad$ information centrality

- $\quad$ betweenness centrality

Besides the described knowledge characteristics, these three measurements have been selected, as they assume shortest paths or 'trails'. In the advice network of Case 1, it was not assumed that knowledge would be transferred twice along the same 
connection, which is the assumption underlying 'walk-based' network flows. In this regard, two other well-known centrality measures, degree centrality and eigenvector centrality, were not further considered in this context, as they only support 'walkbased' network flows.

Closeness centrality and information centrality also imply the appropriate assumption for the kind of trajectory, namely replication (serial or parallel). In contrast to these two measures, betweenness centrality considers the transfer rather than the replication of knowledge. This refers to the second type of knowledge important to this study: explicit electronically unavailable knowledge. In particular, flow betweenness, which is a variant of betweenness centrality, has been selected for Case 1. In contrast to betweenness centrality, flow betweenness considers 'trails' rather than just shortest paths.

\section{Closeness centrality measure}

Closeness centrality measure is typically interpreted as an index of the expected time until something flowing through the network arrives (Borgatti, 1995). Therefore, a high closeness index indicates an actor who receives, for example, new knowledge earlier than others in the network. This is particularly important for innovative R\&D networks. Apart from driving innovation, a high closeness may also benefit organisations in terms of receiving knowledge earlier for knowledge reuse.

In terms of network typology, closeness centrality assumes shortest paths as well as replication and transfer of things that flow in the network. Therefore, this measure considers both types of knowledge relevant to Case 1 (explicit electronically available and unavailable knowledge) as long as this knowledge is transferred along the shortest path in the advice network. Closeness centrality measures focus on the position of an organisation in the network. 
In addition, this measure does not symmetrise the data, which means it considers the direction of knowledge transfer (or initiating and receiving relationships). Therefore, the closeness measure computes separate in-closeness (received relationships/knowledge requests) and out-closeness (initiated relationships/knowledge requests) for directed data. Total closeness centrality sums up in- and out-closeness (Everett \& Borgatti, 2008). However, closeness centrality dichotomises the valued data along ties, which means it does not consider different levels of tie strength. Table 17 shows the results of the closeness centrality measure for the different groups of organisation in Case 1.

Table 17: Closeness centrality per group of organisation in Case 1

\begin{tabular}{|l|c|c|c|}
\hline Group of organisation & $\begin{array}{c}\text { In-closeness } \\
\text { centrality score }\end{array}$ & $\begin{array}{c}\text { Out-closeness } \\
\text { centrality score }\end{array}$ & $\begin{array}{c}\text { Total closeness } \\
\text { centrality score }\end{array}$ \\
\hline CRIs & 100.00 & 33.33 & 133.33 \\
\hline Private businesses & 100.00 & 33.33 & 133.33 \\
\hline Local government & 100.00 & 33.33 & 133.33 \\
\hline Universities & 20.00 & 50.00 & 70.00 \\
\hline Central government & 20.00 & 50.00 & 70.00 \\
\hline
\end{tabular}

CRIs, private businesses and local government organisations with a score of 100.00 showed the highest centrality for incoming relationships and an average score of 33.33 for initiating relationships with other organisations. Universities and central government had low centrality scores for both incoming and initiating relationships. These results identified the CRIs, private businesses and local government as the domain knowledge leaders for Case 1 with the highest total closeness centrality scores. These three key players were the best connected in terms of receiving important knowledge at an early stage.

As social relationships among organisations were characterised by their different levels of tie strength, it is a considerable disadvantage of closeness centrality to dichotomise these valued data. Another measure - information centrality - promises more accurate indications of centrality in knowledge networks, as it considers these valued ties. 


\section{Information Centrality measure}

The information centrality measure refers to the level of ability to communicate (Stephenson \& Zelen, 1989). Therefore, it specifically takes the characteristics of information/knowledge into account. Stephenson and Zelen's (1989) concept suggests that the more central a participant is in the network, the higher their ability to transmit or communicate.

Actors with higher information centrality are predicted to have greater control over the flow of information within a network; highly information-central individuals tend to have a large number of short paths to many others within the social structure ("infocent package:sna R Documentation: Find Information Centrality Scores of Network Positions Description”, 2009). In addition, information centrality takes into account the actual strength of ties between actors (it does not binarise data). The valued tie strength data underlying this measurement are attached in Appendix 8.

Table 18 depicts the Information Centrality measures for the groups of organisations in this advice network.

Table 18: Information centrality per group of organisation in Case 1

\begin{tabular}{|l|r|}
\hline Group of organisation & Actor Information Centrality \\
\hline CRIs & 25.45 \\
\hline Private businesses & 24.63 \\
\hline Local government & 24.60 \\
\hline Central government & 19.35 \\
\hline Universities & 8.18 \\
\hline
\end{tabular}

CRIs, local government and private businesses again had the highest ranked centrality score, whereas universities had the lowest. Compared to the results of closeness centrality, one main difference could be identified: in contrast to equal closeness values, central government organisations had a higher Information Centrality than universities. Overall, the results of information centrality confirmed the ranked list of the three more central groups of organisation resulting from the closeness centrality 
measurement. This is an unexpected result, as closeness centrality does not consider different levels of tie strength compared to information centrality.

Closeness centrality and information centrality assume that knowledge is replicable in the form of explicit electronically available knowledge. As a second type of knowledge (explicit electronically unavailable) was important to several participants in Case 1, flow betweenness centrality was conducted which assumes the transfer of knowledge (instead of replication) as well as 'trails' in a network.

\section{Flow betweenness centrality}

In this study, actors might have used all of the pathways connecting them ('trails'), rather than just the shortest paths (geodesic paths). Accordingly, flow betweenness (Freeman et al., 1991) does not assume shortest paths only, but does assume that no actor is visited more than once ('trails'). As a result, CRIs had the highest score (5.03), local government came second (1.93) and private businesses third (0.75). Universities and central government were the least central organisations with zero values. This indicates that these two types were not on the shortest path between any two other groups of organisation.

Table 19: Flow betweenness centrality scores per group of organisation in Case 1

\begin{tabular}{|l|c|}
\hline Group of organisation & Flow betweenness \\
\hline CRIs & 5.03 \\
\hline Local government & 1.93 \\
\hline Private businesses & 0.75 \\
\hline Universities & 0 \\
\hline Central government & 0 \\
\hline
\end{tabular}

Flow betweenness uses binarised data, which means that it does not consider different levels of tie strength for identifying the most central groups of organisation in the 
network. In this regard, these results may be best compared to the closeness centrality results which are also based on binarised data.

In summary, these three centrality measurements appeared of varying suitability according to the specific types of knowledge transferred in the network of Case 1. For explicit electronically available knowledge, closeness and information centrality were the most appropriate measures. These measures identified the most influential group of organisation, which was the CRI group. Thus, CRIs were the group of organisation that received new or reusable knowledge earliest. This may drive innovation, and it could also be a competitive advantage when applying for new projects.

For explicit electronically unavailable knowledge, such as books and documents however, flow betweenness was employed as the most appropriate measure, as it assumes the transfer (instead of replication) of knowledge and the existence of trails rather than just the shortest paths in a network. Flow betweenness also revealed the CRIs as the most influential. The group of CRIs was therefore the most powerful group of organisation for controlling knowledge transfer in the network. This might be an ideal position for transferring knowledge, for example, in the form of learning initiatives.

In regard to the most appropriate centrality measure for this knowledge network, the researcher suggests information centrality, as it considers replicable knowledge, which was the most important type (electronically available knowledge) in Case 1. In addition, the information centrality measurement considers different levels of tie strength among the groups of organisation.

Two issues were recognised regarding the centrality measurements undertaken. Unexpectedly and despite their various underlying assumptions, the three centrality measures conducted revealed similar results in terms of the ranking of groups of organisation. In addition, the number of study participants per group of organisation appears to have influenced the final ranking. Thus a lower number of study 
participants appeared to lead to a lower centrality score (e.g. local government, universities).

Further, only an organisation of the CRIs group was the provider of professional workshops/seminars in the domain of knowledge in Case 1 (hydrology). This might have influenced their highly ranked position in the centrality measures, especially because underlying data were partly collected from workshop attendees.

Besides these structural aspects of centrality in the Case 1 network, the level of interaction in the network appears significant. Therefore, Section 4.3.4 outlines descriptive statistics representing these levels of interaction.

\subsubsection{The level of interaction}

Descriptive statistics of network data describe the distribution of relational characteristics, such as the level of interaction, among actors. The descriptive statistics, as shown in Table 20, were derived from the information centrality measure, as it was considered the most appropriate measure for this study's context. Results are based on valued data. In addition, the network centralisation index was derived from flow betweenness centrality, as closeness and information centrality do not provide this measure. Table 20 consists of descriptive statistics presenting interactions (i) among all organisations in the social network during the last year.

Table 20: Descriptive statistics for Case 1

\begin{tabular}{|c|c|}
\hline \multicolumn{2}{|l|}{$\begin{array}{l}\text { Descriptive statistics for each measure } \\
\text { Summary statistics: Tie (i) }\end{array}$} \\
\hline Mean & 1.74 \\
\hline Std Dev & 5.63 \\
\hline $\begin{array}{c}\text { Coefficient of variation (standard } \\
\text { deviation / mean x 100) }\end{array}$ & 323.56 \\
\hline Variance & 31.74 \\
\hline Minimum & 0.000 \\
\hline Maximum & 24.08 \\
\hline Network centralisation index & $1.14 \%$ \\
\hline
\end{tabular}


The data described in Table 20 indicate a high variation in activity levels expressed by a high variance measure (31.74). In addition, the coefficient of variation equals 323.56 and thus presents a high variation as a percentage of the average score. Interactions in this network included a large number of low level interactions. These interactions are corresponding to weak ties (Granovetter, 1973). In addition, there were a smaller number of ties (including five key organisations) with a higher level of interaction indicating strong ties. These five key organisations may also represent the preferred sources for domain knowledge in the professional sector of hydrology.

The network centralisation index (1.14\%) of this social network is very low compared to a pure star network (Hanneman, 2005). This shows that there was no single organisation in the network that controlled the network by itself. This indicates a decentralised inter-organisational network. The network centralisation index is higher within the core group of five key organisations (39.75\%), and also when measuring it for the aggregated groups of organisation (36.35\%), but still signals a decentralised network.

In order to present more precise results, Tables 20 and 21 depict univariate statistics for the key organisations. With having valued data (represent the levels of contact frequency for giving and seeking advice activities and the number of study participants per organisation in this study) the mean represents the average strength of ties, rather than the probability of ties (Hanneman, 2005). A summary of descriptive statistics for each organisation's sending and receiving activities provides a better insight into the network's activities.

These sending and receiving activities can be regarded as basic demographic features. Individual organisations may be 'sources' of relationships (organisations that initiate a lot of relationships), 'sinks' (organisations that receive relationships, but do not initiate them), or both (Hanneman, 2005). These types of very basic differences among organisations' immediate connections may be critical in explaining how they view the remaining network, and how the rest of the network views them. 
Table 21: Summary of univariate statistics for key organisations by column (key sinks)

\begin{tabular}{|l|c|c|c|c|c|}
\hline & Private business & $\begin{array}{l}\text { Regional } \\
\text { Council 1 }\end{array}$ & CRI 2 & CRI 1 & $\begin{array}{l}\text { Regional } \\
\text { Council 2 }\end{array}$ \\
\hline Mean & 5.25 & 11.25 & 6.50 & 6.50 & 3.00 \\
\hline Std Dev & 5.54 & 9.60 & 4.50 & 3.57 & 4.64 \\
\hline Variance & 30.69 & 92.19 & 20.25 & 12.75 & 21.50 \\
\hline Minimum & 0.00 & 1.00 & 2.00 & 2.00 & 0.00 \\
\hline Maximum & 13.00 & 24.00 & 11.00 & 10.00 & 11.00 \\
\hline
\end{tabular}

The presentation of the data revealed a pattern of sink behaviour (Hanneman 2005, p.43) represented by the fact that some organisations in the network were the target of directed interaction. In this sense a higher mean value indicates an organisation that received more requests for relationships than they initiated (Hanneman, 2005). A clear sink pattern was revealed which suggests Regional Council 1 as the key sink in this network. In addition, the two CRIs and the private business also had a reasonable number of receiving relationships. In contrast to the key sinks, the organisations that were key sources are illustrated by high mean values in the following table.

Table 22: Summary of univariate statistics for key organisations by row (key sources)

\begin{tabular}{|l|l|l|l|l|l|}
\hline & Private business & $\begin{array}{l}\text { Regional } \\
\text { Council 1 }\end{array}$ & CRI 2 & CRI 1 & $\begin{array}{l}\text { Regional } \\
\text { Council 2 }\end{array}$ \\
\hline Mean & 9.00 & 8.75 & 1.75 & 11.75 & 1.25 \\
\hline Std Dev & 9.43 & 4.60 & 1.78 & 3.27 & 0.83 \\
\hline Variance & 89.00 & 21.19 & 3.19 & 10.69 & 0.69 \\
\hline Minimum & 0.00 & 1.00 & 0.00 & 8.00 & 0.00 \\
\hline Maximum & 24.00 & 13.00 & 4.00 & 17.00 & 2.00 \\
\hline
\end{tabular}

Table 22 shows CRI 1, private business and Regional Council 1 as the key sources with the highest mean values. The private business had a mean for initiating ties of 9.00 which was high compared to the mean value of 5.25 for receiving ties. This result shows that the private business organisation may need to initiate relationships more often than larger organisations like Regional Council 1 or CRI 1. This was confirmed by the results that showed Regional Council 1 received more relationships than it initiated. This again shows a high variability of interactions across organisations. 
This section has outlined univariate statistics to describe the levels of interaction in the inter-organisational network of Case 1. Key sinks and key sources were identified. In addition, summaries of the univariate statistics by the group of organisation level can be found in Appendix 10.

\subsection{Conclusion and discussion}

\section{Summary}

This chapter described and interpreted key results of Case 1. Key findings were related to the strength of relationships and the direction of knowledge transfer as well as the types of knowledge transferred. Eigthy employees from twenty-seven organisations that were active in the professional field of hydrology in New Zealand took part in Case 1. These organisations were aggregated into groups of organisations, namely CRIs, central government, local government (incl. regional councils), universities and private businesses. The direction of knowledge transfer and tie strength among (groups of) organisations was explained and illustrated. As a key result, the important roles of CRIs, private business and local government were identified. These groups of organisations played a significant role for co-ordinating and controlling knowledge transfer in the network of Case 1.

Two different types of knowledge were the most important ones to Case 1 participants:

- electronically accessible references to other sources of knowledge

- electronically accessible formal knowledge

Examples for electronically accessible formal knowledge were research papers in .pdf format, reports, journal articles and conference papers. In addition, electronically 
unavailable knowledge types also appeared significant in the context of this study. These were

- informal how-to knowledge

- meta-knowledge (particularly experts' contact details)

- informal factual knowledge

Examples for these electronically unavailable knowledge types were verbal advice, unpublished data, personal observations and opinions, experiences and suggestions.

In addition, structural aspects regarding the centrality of organisations and the level of interaction in the knowledge network were explored in depth. Closeness centrality, information centrality and flow betweenness centrality analyses were conducted. The more central groups of organisations were CRIs, local governments and private businesses.

In line with these results, this chapter helped answer the first two sub-questions of this research in regard to Case 1's results:

1) What are the key structural properties of advice networks and their implications for knowledge transfer?

2) Which types of knowledge are transferred in the advice networks?

The key results of this case are briefly discussed in the following paragraphs. 


\section{Discussion}

Study results

In Case 1, different levels of tie strength were related to the types of knowledge transferred in the network. Results showed that the types of knowledge, for example electronically available meta-knowledge and electronically unavailable informal knowledge, varied between strong and weak ties. Hansen (1999) differentiated knowledge by its complexity levels. He argued for the transfer of different knowledge in weak and strong ties. Although Reagans and McEvily (2003) downplayed the importance of the impact of tie strength on the type of knowledge transferred, they also stated that strong ties facilitated the transfer of tacit knowledge more than they facilitated the transfer of codified knowledge.

In terms of media use for knowledge transfer and learning, another key result of Case 1 indicated the interest of inexperienced workers in the use of social software. One explanation for this preference might be the fact that they are typically younger than their experienced colleagues; and social software is the type of communication medium used more often by the so-called Net generation born between 1980 and 1999 (Kupperschmidt, 2001).

A contribution to knowledge has been made in relation to applying appropriate centrality measures to knowledge networks. The most appropriate SNA centrality measures have been identified for different types of knowledge transfer in a network considering the two dimensions: ways that traffic (knowledge) may follow and its method of spread. These considerations relate to current literature addressing these issues (Borgatti, 2005).

The selection of the most suitable centrality measures has been guided by the types of knowledge most frequently used for knowledge transfer by participants in Case 1. These types were explicit electronically available knowledge and explicit but electronically unavailable knowledge. For explicit electronically available 
knowledge, which is replicable, closeness centrality and information centrality were the most appropriate measures. For explicit electronically unavailable knowledge, such as books and documents however, flow betweenness centrality has been employed as the most appropriate measure.

However, in regard to the overall most appropriate centrality measure for this study's context, the researcher has suggested information centrality, as it considered replicable knowledge, which was the type used most often in the network. The information centrality measurement has also considered different levels of tie strength among the groups of organisations. This contribution to the SNA and knowledge management literature has improved the understanding of inter-organisational knowledge transfer structures using SNA which has been a major goal of this study.

One managerial implication is related to the use of ICT to support knowledge transfer. Besides using the email newsletter of a related professional society, study participants did not have any opportunity to connect via a domain-specific online platform to share their knowledge and experiences. In the context of networking benefits, ICT-supported collaboration may add value to current face-to-face workshops, as collaborative ICT facilitates the creation of new relationships (Haythornthwaite, 2005) and helps maintain existing ones.

\section{Possible managerial implications}

The level of interaction in the inter-organisational network of Case 1 was investigated using univariate statistics. Key players were identified: CRIs and local government were the key sinks (receiving relationship requests). The private businesses and CRIs were the key sources (initiating relationships). In addition, universities and central government were active in initiating relationships for knowledge transfer. As CRIs were key sinks and key sources, they represented knowledge brokers in the domain of hydrology. Together with the three more powerful organisation groups - CRIs, private business and local government - these findings on the levels of interaction may be a significant source of managerial implications. Social network analysis 
results have previously been used in the organisational context (e.g. Cross, Laseter, Parker \& Velasquez, 2006; Liebowitz, 2005). The knowledge about key players and their characteristics may be used to inform future business decisions about interorganisational knowledge transfer and learning.

Besides CRIs, local government also had a very powerful position in the network. Nevertheless, a CRI was the only provider of professional workshops in the domain of hydrology. Trainers from CRIs may provide most of the value, as these organisations represent key knowledge brokers in the field, and therefore may be able to draw on a wealth of knowledge and long-term experience. However, managerial implications could include a rethinking of the organisation of workshops/seminars in regard to the appointment of trainers. Besides CRI employees, trainers of workshops in the hydrology domain could also include staff from local government. Local government employees would provide additional value as workshop trainers, as they work at the intersection of government, research and the resolution of problems in local areas. For example, applying government policies and research outcomes to project work, may provide additional valuable perspectives to workshop participants.

From an even more important perspective, this study revealed that significant research knowledge that is not part of the final product has often not been saved nor provided to other people electronically. The introduction of an online platform and managerial efforts to support saving this data and knowledge could have enormous advantages for future projects, as it might help reuse knowledge and thus prevent reinventing the wheel. Although this aspect appears to be more an organisational than an inter-organisational issue, it is a significant issue that needs managerial consideration.

While this chapter has presented the Case 1 results, the next chapter depicts the findings of Case 2. Minor changes made to the data collection of Case 2 after Case 1 were addressed in Section 3.6. However, these changes had no major impact on the analysis of data in Case 2. 


\section{Case 2 results}

This chapter reports on key results of Case 2 as part of this multiple case study. Firstly, it describes the survey participants' characteristics and their types of knowledge transferred. Then, key findings in regard to structural properties of this social network are outlined. This includes tie strength and the levels of interaction. In addition, centrality measures were conducted to identify the most influential organisations in the network. These key results are based on data collected from workshop participants (initial observation and final follow-up questions) and survey participants. Finally, this chapter concludes with a summary and a brief discussion of the key findings.

\subsection{Study participants characteristics}

This section describes the characteristics of study participants who took part in the online survey in Case 2. Eighty-one study participants from 22 organisations that were active in the professional field of marine biology in New Zealand took part. The marine biology topic was taken as a boundary for a social network including people who were working at diverse organisations, but had the same work interests and therefore gave advice to and sought advice from peers at the workplace.

Two key organisations and three key groups of organisation in the NZ environmental R\&D sector were initially identified, prior to the SNA data collection. The two key organisations were a CRI and a private medium-sized research and consulting organisation. The three key groups of organisation were represented by local government, universities and central government.

All individual organisations could finally be aggregated at a level that represented specific groups of organisation which were: 
CRIs,

$>$ central government agencies,

$>$ local government agencies,

$>$ universities,

$>$ private businesses,

$>$ not-for-profit organisations (NPOs) and

$>$ others (anonymous study participants or organisations that did not fit into one of the other categories)

Apart from the 'others' category, these six groups of organisation showed typespecific characteristics in the form of ownership and core business activities, as summarised in Table 23.

Table 23: Characteristics of key groups of organisations in Case 2

\begin{tabular}{|l|l|l|}
\hline Group of organisation & Ownership & Core business activities \\
\hline CRIs & Public & $\begin{array}{l}\text { Basic research, consulting, } \\
\text { publications }\end{array}$ \\
\hline Local government & Public & $\begin{array}{l}\text { Applied research, consulting, } \\
\text { publications }\end{array}$ \\
\hline Universities & Public & $\begin{array}{l}\text { Basic research, applied Research, } \\
\text { publications }\end{array}$ \\
\hline Private businesses & Private & $\begin{array}{l}\text { Applied research, consulting, } \\
\text { publications }\end{array}$ \\
\hline Central government & Public & $\begin{array}{l}\text { Consulting and monitoring, } \\
\text { reporting and publications }\end{array}$ \\
\hline NPOs & N/a & Reporting, monitoring \\
\hline
\end{tabular}

All participating organisations were at least partly active in the same professional field (marine biology). Some key organisations were more specialised in marine biology than others. Individual study participants had various roles in the field ranging from aquaculture scientists to marine ecologists to $\mathrm{PhD}$ students in the marine biology field. However, most participants were researchers and scientists. Workshops and seminars that were offered to the public were held by only one CRI.

In terms of contact frequency, inter-organisational knowledge transfer happened mostly with a 3- to 6-monthly frequency, whereas a higher contact frequency (monthly) existed, for example, between university and CRI types. Intraorganisational knowledge transfer always showed a higher frequency than interorganisational knowledge transfer. Seeking and giving advice were often equally important for study participants within one organisation and also in the interorganisational context. 
Inter-organisational knowledge transfer between these groups of organisations appeared to happen either in formal business relationships, in publicly offered seminars, workshops and conferences, or in close social relationships. In addition, there was an established network for knowledge transfer between regional councils as mentioned earlier. Moreover, longstanding social relationships between some CRIs and some universities seem to have supported informal knowledge transfer between students and CRI staff in the marine biology field.

Not all 81 participants answered each question. However, a majority of 67 participants answered the two survey questions on contact frequencies. These were of particular interest to the SNA part of this chapter. Participants tended to opt out of the survey more often in the second part than in the first part. As in Case 1, this may lead to the conclusion that they considered the survey was too long. However, no particular non-respondent bias could be identified.

Table 24 depicts the number of study participants who answered the survey's questions on the frequencies of advice giving and seeking activities. Table 24 shows the number of study participants from each key organisational type.

Table 24: No of study participants per key organisational type in Case 2

\begin{tabular}{|l|c|}
\hline \multicolumn{1}{|c|}{ Group of organisation } & No. of study participants \\
\hline Universities & 17 \\
\hline Private businesses & 16 \\
\hline CRls & 15 \\
\hline Central government & 12 \\
\hline Local government & 3 \\
\hline Others & 3 \\
\hline NPOs & 1 \\
\hline All organisations & 67 \\
\hline
\end{tabular}

The low number (1) for the NPO may be a result of not including it as a key (group of) organisation prior to data collection. The low number of participants from local government may reflect a low number of people working on marine biology topics in regional councils. In addition, there were only a few individuals from local 
government who were members of the professional society that was contacted for survey distribution in Case 2. More responses were received from universities, private businesses, CRIs and central government agencies.

Bearing in mind the membership figures provided by the professional societies contacted for data collection, the overall response rate could be calculated at around 22 percent. As in Case 1, due to a pre-selection of certain (groups of) organisations for data collection and limited control over the actual organisational affiliation of participants in Case 2, this response rate remains an approximate figure.

Overall, a slightly higher number of men $(55.6 \%)$ than women $(44.4 \%)$ participated in the survey. Supporting data can be found in Appendix 11. Overall, there were more females who were new at their job than males as indicated in Figure 15.

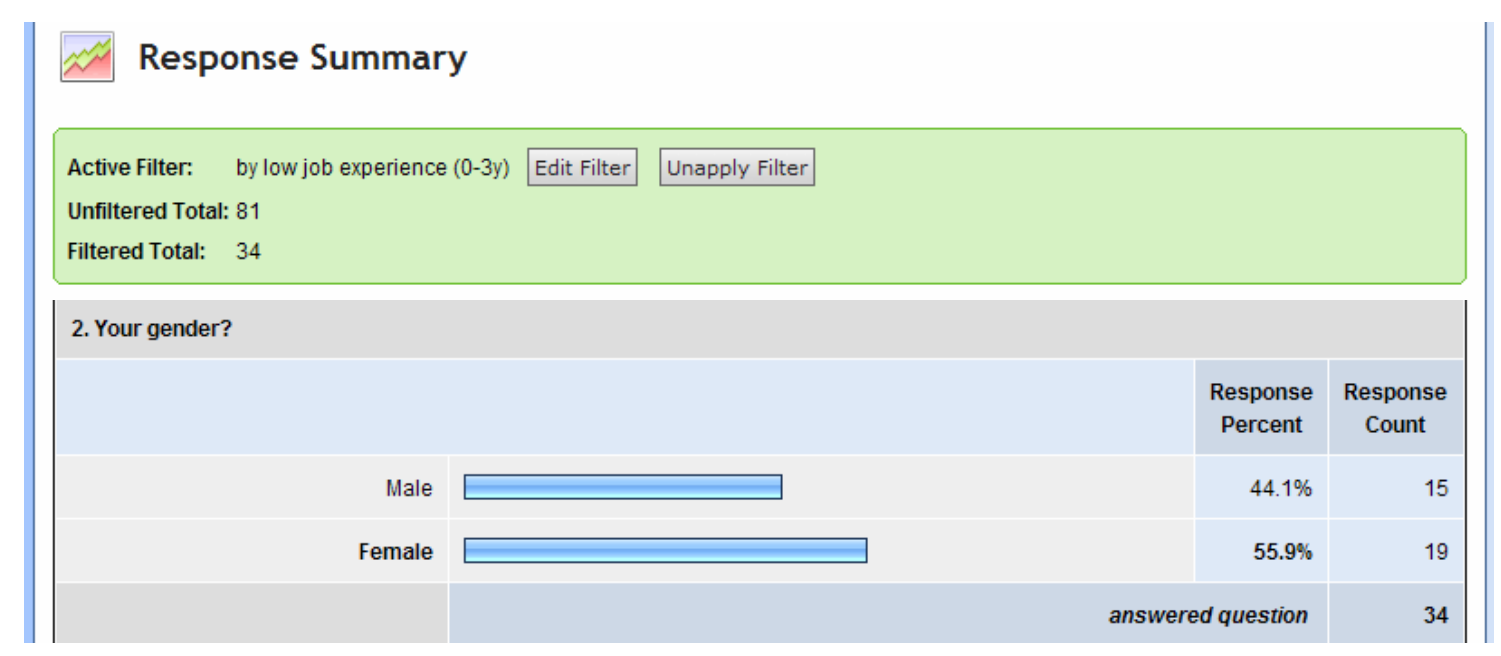

Figure 15: Job beginners (0-3 years) by gender in Case 2

The following figure shows the job experience of study participants. A relatively high number of new employees (0-3 years in their job) and people with 4 to 10 years of job experience participated. This question on job experience was answered by all 81 participants. 


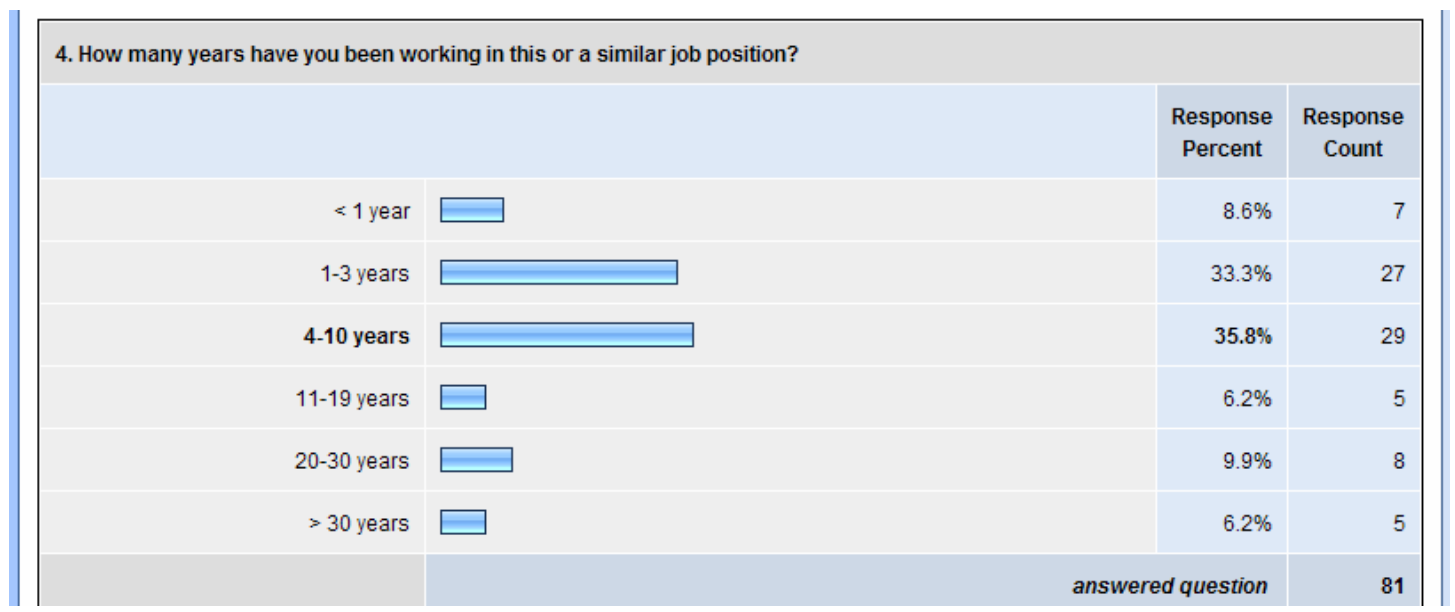

Figure 16: Study participants by job experience in Case 2

The following section introduces the possible relationships between these study participants.

The relationships between different groups of organisations

This section describes the relational characteristics among the key groups of organisations. As all organisations belonged to the same professional field (marine biology), one would expect a number of formal business relationships such as alliances as well as various informal relationships, for example through friendships or acquaintanceships. In Case 2, the co-existence of formal and informal relationships was confirmed by one participant from the CRI in the follow-up questions to the online survey (Phase 3):

"Great range of different types of interactions - some formal, some informal" (Participant 1).

There was also a critical comment from the same participant:

"Sometimes these organisations are forced into competition (which can be damaging to flows of information) because of the science funding model in NZ" (Participant 1). 
These comments indicate an environment of simultaneous competition and collaboration in an inter-organisational context. In particular, universities, the CRI and the private business were involved in partner relationships that might have been affected by competition. These organisations also acted as domain knowledge specialists in the field of marine biology.

Case 2 revealed the following possible relationships between participating key groups of organisations:

Table 25: Possible relationships between groups of organisation in Case 2

\begin{tabular}{|c|c|}
\hline $\begin{array}{ll}> & \text { CRIs - local government } \\
> & \text { CRIs - private businesses } \\
> & \text { CRIs - central government } \\
> & \text { CRIs - NPOs } \\
> & \text { CRIs }- \text { universities } \\
> & \text { CRIs }- \text { others }\end{array}$ & $\begin{array}{l}>\text { Private businesses - local government } \\
>\text { Private businesses - NPOs } \\
>\text { Private businesses - others }\end{array}$ \\
\hline $\begin{array}{ll}> & \text { Universities - local government } \\
> & \text { Universities - private businesses } \\
> & \text { Universities - central government } \\
> & \text { Universities - NPOs } \\
> & \text { Universities - others }\end{array}$ & $\begin{array}{l}>\text { Local government }- \text { NPOs } \\
>\quad \text { Local government }- \text { others }\end{array}$ \\
\hline $\begin{array}{ll}> & \text { Central government }- \text { local government } \\
> & \text { Central government }- \text { NPOs } \\
> & \text { Central government }- \text { private businesses } \\
> & \text { Central government }- \text { others }\end{array}$ & $>$ NPOs - others \\
\hline
\end{tabular}

These possible inter-organisational relationships represent 21 different kinds of relationships. Moreover, when considering the diverse contact frequencies (once every week, once every month, once every three months, once every six months, or never), the variety of relationships multiplies.

In their social relationships, Case 2 participants transferred various types of knowledge that were an important factor influencing knowledge transfer in interorganisational advice networks. 


\subsection{Knowledge characteristics}

Knowledge characteristics were a significant factor in relation to inter-organisational knowledge transfer in the advice network of Case 2. This section outlines the types of knowledge focussed on in Case 2. The importance of various types of knowledge to different types of social relationships is explained.

In Case 2, knowledge was distinguished by nine diverse types of knowledge. Knowledge was firstly differentiated into electronically accessible and electronically inaccessible knowledge. This distinction has previously also been made by Maier et al. (2005).

In addition to Case 1, Case 2 differentiated moreover between two types of electronically inaccessible knowledge: non-codified or tacit knowledge (only available in people's minds) and paper-based knowledge (e.g. old documents, books). For data collection, three different types of knowledge were the result of this differentiation:

$>$ electronically accessible knowledge,

$>$ electronically inaccessible (but codified) knowledge, and

$>$ electronically inaccessible (non-codified/tacit) knowledge.

Although tacit knowledge is defined in knowledge management literature as having several important dimensions such as being intuitive, gained through experience and difficult to codify (Section 2.1.2), it was briefly explained to study participants as 'non-codified content that refers to the knowledge that people carry in their minds, but which is not written down (as codified knowledge is)'. It was hoped that this very simplified definition of tacit knowledge would be easy for study participants who were typically not familiar with concepts of KM to understand. However, for the further interpretation of results in this thesis, electronically inaccessible non-codified knowledge needs to be distinguished from electronically inaccessible tacit knowledge, as non-codified knowledge may contain only a small portion of the 
characteristics of tacit knowledge (e.g. difficult to codify). Thus, the term 'noncodified' will replace the term 'tacit' when necessary in the following descriptions.

Apart from the electronic availability and the differentiation into codified and noncodified knowledge, six other types of knowledge were considered. Knowledge was differentiated into meta-knowledge (experts' contact details, references to other sources of information), formal knowledge (facts, how-to) and informal knowledge (facts, how-to).

The types of knowledge that were most important to study participants are shown in relation to weak and strong ties in Table 26 and can be categorised as:

- informal knowledge (factual and how-to knowledge, both electronically inaccessible),

- formal knowledge (factual and how-to, both electronically accessible), and

- meta-knowledge (references to other sources of knowledge and experts' contact details, both electronically accessible).

Electronically accessible but codified knowledge was less favoured. Specific types of knowledge were transferred in different kinds of relationships with different tie strength and knowledge transfer directions

Table 26 summarises the key results for giving and seeking advice activities in strong and weak social relationships between organisations. Sixty-seven study participants from the key groups of organisation considered the following types of knowledge to be significant for their inter-organisational knowledge transfer. Electronically inaccessible knowledge is highlighted in bold. Supporting data can be found in Appendix 12. 
Table 26: Types of knowledge transferred in Case 2

\begin{tabular}{|l|c|c|c|c|c|c|}
\hline & $\begin{array}{l}\text { Electron. } \\
\text { accessible } \\
\text { formal } \\
\text { documents } \\
\text { (facts) }\end{array}$ & $\begin{array}{l}\text { Electron. } \\
\text { accessible } \\
\text { formal } \\
\text { documents } \\
\text { (how-to) }\end{array}$ & $\begin{array}{l}\text { Electron. } \\
\text { accessible } \\
\text { references to } \\
\text { other } \\
\text { sources of } \\
\text { knowledge }\end{array}$ & $\begin{array}{l}\text { Electron. } \\
\text { accessible: } \\
\text { expert's } \\
\text { contact } \\
\text { details }\end{array}$ & $\begin{array}{l}\text { Electron. } \\
\text { inaccessible } \\
\text { Inon-codified } \\
\text { informal how- } \\
\text { to knowledge }\end{array}$ & $\begin{array}{l}\text { Electron. } \\
\text { inaccessible/ } \\
\text { non-codified } \\
\text { informal } \\
\text { factual } \\
\text { knowledge }\end{array}$ \\
\hline $\begin{array}{l}\text { Giving } \\
\text { strong ties }\end{array}$ & $\mathrm{X}$ & $\mathrm{X}$ & $\mathrm{X}$ & $\mathrm{X}$ & $\mathbf{X}$ & $\mathbf{X}$ \\
\hline $\begin{array}{l}\text { Giving } \\
\text { weak ties }\end{array}$ & $\mathrm{X}$ & $\mathrm{X}$ & $\mathrm{X}$ & $\mathrm{X}$ & $\mathbf{X}$ & $\mathbf{X}$ \\
\hline $\begin{array}{l}\text { Seeking } \\
\text { strong ties }\end{array}$ & $\mathrm{X}$ & $\mathrm{X}$ & $\mathrm{X}$ & $\mathrm{X}$ & $\mathbf{X}$ & \\
\hline $\begin{array}{l}\text { Seeking } \\
\text { weak ties }\end{array}$ & $\mathrm{X}$ & $\mathrm{X}$ & $\mathrm{X}$ & $\mathrm{X}$ & & \\
\hline
\end{tabular}

The results in Table 26 indicate that two types of knowledge were not electronically accessible for a large number of study participants: informal how-to knowledge and informal factual knowledge. Study participants in strong relationships transferred mostly the same types of knowledge as study participants in weaker relationships. However, for giving advice activities, study participants also transferred informal factual knowledge that was not available electronically or in a paper-based format. In summary, six different types of knowledge were significant for inter-organisational knowledge transfer. Two of these six types were electronically inaccessible. Electronically inaccessible knowledge in the form of non-codified knowledge was most important to study participants.

Examples of both non-codified and codified electronically inaccessible knowledge were given by study participants in the follow-up questions to the SNA (Phase 3). Examples of codified knowledge were old papers and old literature which was not held in NZ. Examples of non-codified knowledge were unpublished research material that had not been written down. Examples for tacit knowledge were experiences, opinions and suggestions on a topic. Examples of electronically available knowledge were pdf documents, images and manuscripts.

This section has described which different types of knowledge were transferred throughout the inter-organisational advice network in the professional field of marine biology in New Zealand. Results showed that the types of knowledge varied between 
givers and seekers of knowledge. Moreover, informal how-to and informal factual knowledge were not electronically accessible.

The following section describes the key findings which are related to the structural properties of the advice network in Case 2.

\subsection{Key findings related to structural properties of the advice network}

Key findings show the levels of interaction and tie strength. In addition, appropriate centrality measures were selected to analyse the most central groups of organisations for knowledge transfer in the social network.

\subsubsection{Levels of interaction}

The following descriptive statistics present interactions (i) among all groups of organisation in the social network of Case 2 during the last year. These statistics were derived from the information centrality measure, as it was considered the most appropriate for this study's context. Results are based on valued data. However, the network centralisation index was again (as in Case 1) derived from flow betweenness centrality, as closeness and information centrality do not provide this information. The data described below indicates a high variation in activity levels expressed by a high variance measure (64.16). Data used for these measurements can be found in Appendix 13. 
Table 27: Descriptive statistics of the Case 2 network

\begin{tabular}{|c|c|}
\hline $\begin{array}{l}\text { Descriptive statistics for each measure } \\
\text { Summary statistics: Tie (i) }\end{array}$ \\
\hline Mean & 23.51 \\
\hline Std. Dev. & 8.01 \\
\hline $\begin{array}{c}\text { Coefficient of variation } \\
\text { (standard deviation / mean } \mathrm{x} \\
100 \text { ) }\end{array}$ & 34.07 \\
\hline Variance & \\
\hline Minimum & 64.16 \\
\hline Maximum & 0.00 \\
\hline Network centralisation index & 30.13 \\
\hline
\end{tabular}

Interactions in this network included a low number of low level interactions corresponding to weak ties and five key groups of organisation with a higher level of interaction indicating strong ties. These five key groups of organisation were universities, CRIs, central government, local government and private business. These types, particularly universities, might have represented the preferred sources for domain knowledge in the professional sector of marine biology.

The network centralisation index $(25.32 \%)$ of this social network was relatively low compared to a pure star network with an index of $100 \%$ (Hanneman, 2005). Network centralisation demonstrates the global centrality within a network and measures the degree to which relationships within a network are focused around a single or a few central network members (Freeman, 1979). The low network centralisation index here shows that there was no single organisation in the network that controlled the network by itself. This indicates a decentralised inter-organisational network.

In order to present more precise results, Tables 27 and 28 depict univariate statistics for the groups of organisation. Considering valued data (representing the level of contact frequency and the number of study participants per group of organisation in this study), the mean represents the average strength of ties, rather than the probability of ties (Hanneman, 2005). A summary of descriptive statistics for each organisation's sending and receiving activities provides a better insight into the 
network's activities. These activities included initiating relationships for knowledge transfer or receiving requests for relationships and knowledge transfer.

Results can be compared to the interpretation of the figures illustrating tie strength as outlined in the next section. Table 28 depicts the univariate statistics per group of organisation for receiving relationship requests.

Table 28: Summary of univariate statistics for groups of organisations in Case 2 by column (key sinks)

\begin{tabular}{|l|c|c|c|c|c|c|c|}
\hline & CRIs & $\begin{array}{l}\text { Central } \\
\text { government }\end{array}$ & Universities & $\begin{array}{l}\text { Private } \\
\text { businesses }\end{array}$ & $\begin{array}{l}\text { Local } \\
\text { government }\end{array}$ & NPOs & others \\
\hline Mean & $\mathbf{1 9 . 1 7}$ & $\mathbf{1 1 . 8 3}$ & $\mathbf{2 3 . 6 7}$ & $\mathbf{5 . 6 7}$ & $\mathbf{1 6 . 5 0}$ & $\mathbf{0 . 0 0}$ & $\mathbf{0 . 0 0}$ \\
\hline Std. Dev. & 17.49 & 10.24 & 18.82 & 4.71 & 16.47 & 0.00 & 0.00 \\
\hline Variance & 305.81 & 104.81 & 354.22 & 22.22 & 271.25 & 0.00 & 0.00 \\
\hline Minimum & 0.00 & 0.00 & 1.00 & 0.00 & 0.00 & 0.00 & 0.00 \\
\hline Maximum & 42.00 & 28.00 & 44.00 & 13.00 & 39.00 & 0.00 & 0.00 \\
\hline
\end{tabular}

This presentation of the data revealed a pattern of sink behaviour (Hanneman 2005, p.43) represented by the fact that some groups of organisations in the network were the target of directed interaction. In this sense, a higher mean value indicates a type that received a higher number of requests for relationships (formal or informal) than other groups of organisation (Hanneman, 2005). The key 'sinks' were universities, CRIs and local governments. Central government study participants were also contacted often for knowledge transfer.

The groups of organisation that were 'sources' of relationships rather than 'sinks' are shown in Table 29. The groups of organisation that initiated the most relationships were private business as well as central government. In addition, universities and CRIs were also active in initiating relationships for knowledge transfer.

Table 29: Summary of univariate statistics for groups of organisations in Case 2 by row (key sources)

\begin{tabular}{|l|c|c|c|c|c|c|c|}
\hline & CRIs & $\begin{array}{l}\text { Central } \\
\text { government }\end{array}$ & Universities & $\begin{array}{l}\text { Private } \\
\text { businesses }\end{array}$ & $\begin{array}{l}\text { Local } \\
\text { government }\end{array}$ & NPOs & others \\
\hline Mean & $\mathbf{1 1 . 6 7}$ & $\mathbf{2 3 . 0 0}$ & $\mathbf{1 2 . 6 7}$ & $\mathbf{2 3 . 6 7}$ & $\mathbf{1 . 5 0}$ & $\mathbf{1 . 3 3}$ & $\mathbf{3 . 0 0}$ \\
\hline Std. Dev. & 13.77 & 19.22 & 10.92 & 18.30 & 1.71 & 2.98 & 2.58 \\
\hline Variance & 189.56 & 369.33 & 119.22 & 334.89 & 2.92 & 8.89 & 6.67 \\
\hline Minimum & 0.00 & 0.00 & 0.00 & 0.00 & 0.00 & 0.00 & 0.00 \\
\hline Maximum & 40.00 & 44.00 & 28.00 & 43.00 & 5.00 & 8.00 & 7.00 \\
\hline
\end{tabular}


Overall, universities had a relatively high number of initiating relationships (12.67) as well as a high number of incoming relationships requests (23.67) for knowledge transfer. Thus the group of universities represents a knowledge broker in the field of marine biology.

Linkages that show the direction of knowledge transfer can also be weighted by the level of tie strength. The following section 'Tie strength' explains and describes how tie strength was measured in the inter-organisational context of Case 2.

\subsubsection{Tie strength}

A whole network approach, as explained in the research methods chapter, was used for collecting and visualising tie strength. Tie strength between organisations and/or groups of organisations was measured by contact frequency (once every week, once every month, once every three months, once every six months, or never) and the number of study participants from each organisation/group of organisations. Supporting data are found in Appendix 14. For Case 2, the researcher aggregated some organisations into organisational types. For example, all study participants from regional councils were aggregated at the regional councils' level and all study participants from universities were aggregated at the university level. Some aggregations could be done prior to data collection and others when analysing the data. This appeared to be useful, as it was not entirely clear in the beginning of the study which were the key organisations and how many study participants would come from a single regional council or university for example.

Figure 17 illustrates this advice network showing the key organisations (the CRI and the private business) and the three aggregated groups of organisation prior to data collection (regional councils, universities, and central government). Figure 17 indicates the strength and direction of knowledge transfer within the network. Tie 
strength is illustrated by line thickness. The direction of knowledge transfer shows giving and/or seeking advice and is illustrated by an arrow at the end of the line.

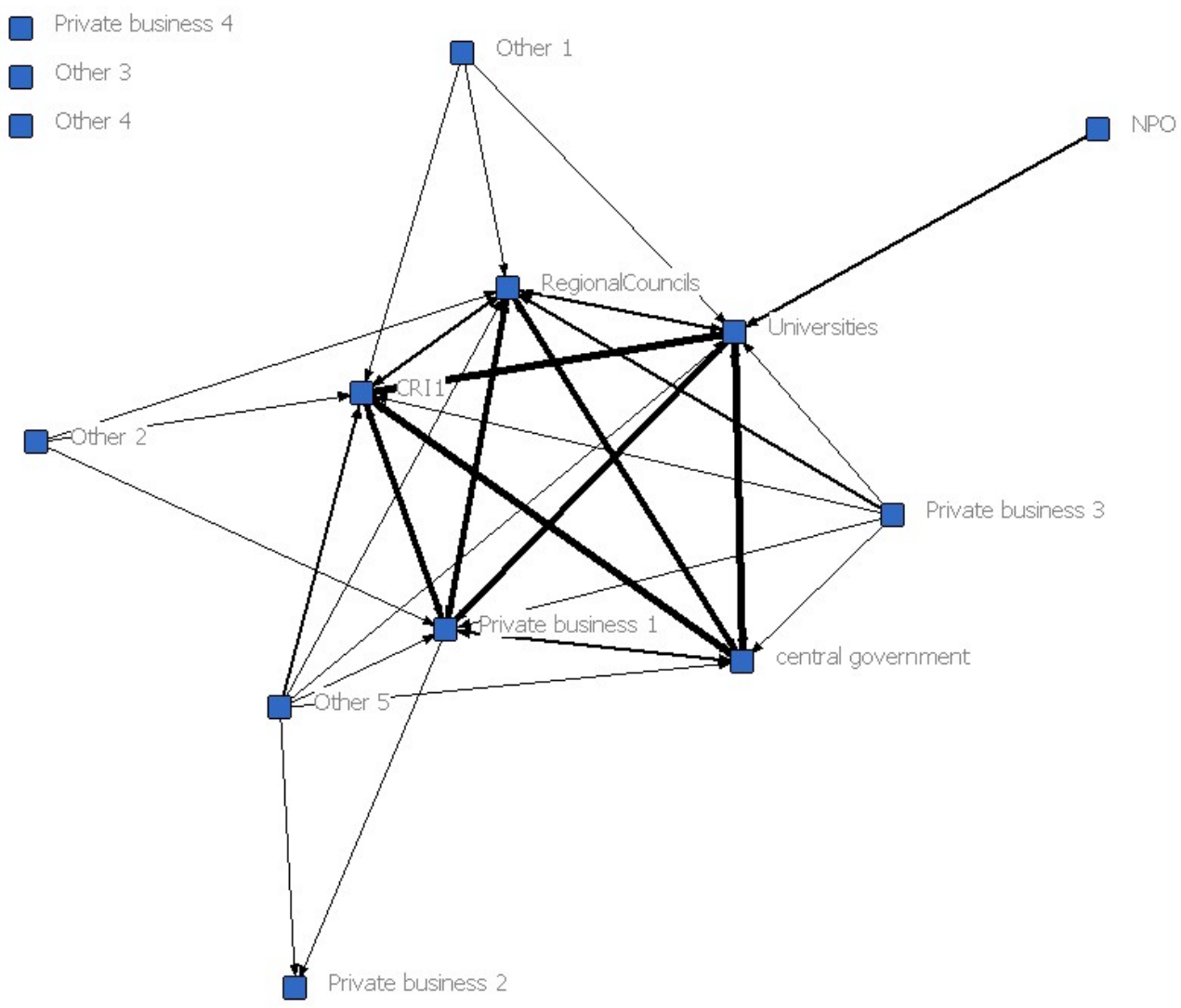

Figure 17: Case 2 advice network (whole network)

Figure 17 presents the tie strength results and illustrates the whole network of fourteen participating organisations or groups of organisations in the marine biology field. They either gave advice to people from other organisations in the network or sought advice from people working at other organisations during the last year. Five key (types of) organisations with a total number of 60 study participants were strongly connected. They can be easily identified by their line thickness in the network. In addition, there are some weak ties (thin lines) with a total number of five organisations comprising seven individual study participants. Three isolated organisations that either indicated intra-organisational knowledge transfer only or none at all could also be identified. In addition, only one person participated from 
each of these three isolated organisations (private business 4, other 3, other 4). Coded data underlying these tie strength measurements can be found in Appendix 14.

The direction of knowledge transfer (giving and/or seeking advice) and the contact frequency as well as the number of study participants were used for the tie strength measure. The values along the lines in Figure 18, for example, demonstrate the tie strength for each direction from each organisation. It is a snapshot that focuses on the stronger relationships presented in Figure 17. Figure 18 uses values for outlining the tie strength among key organisations.

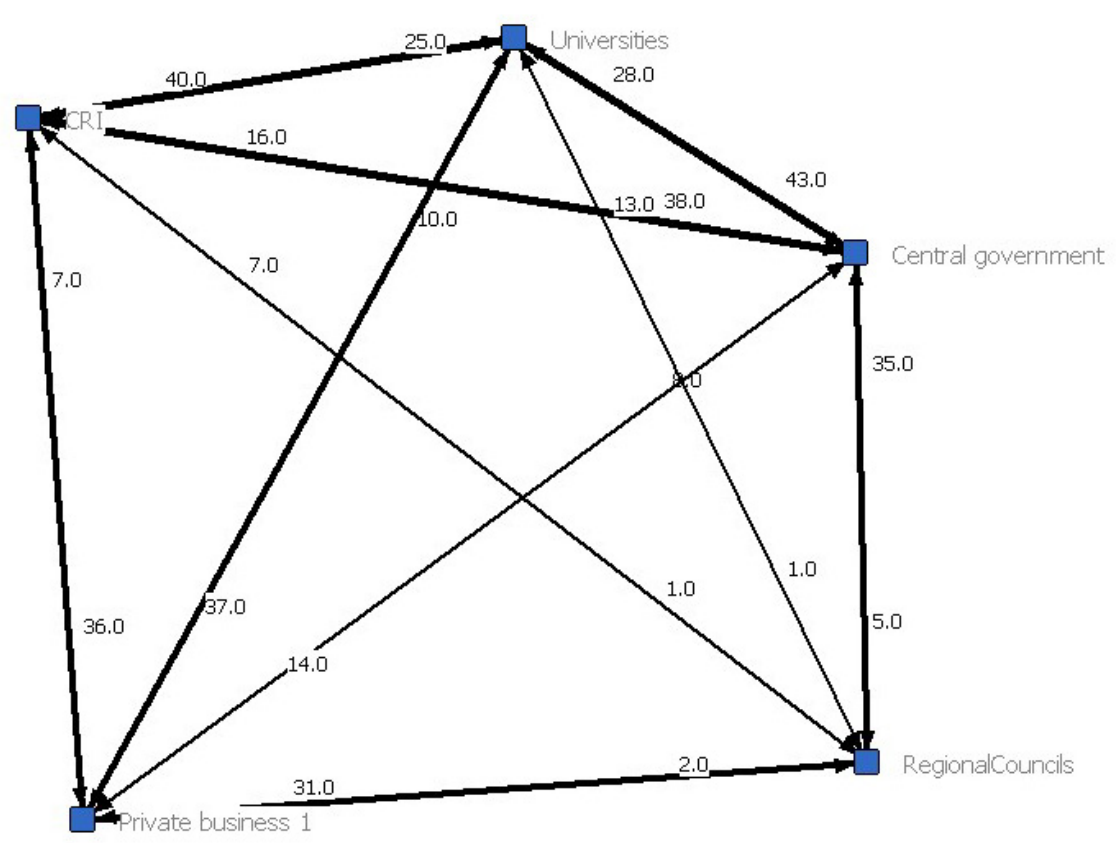

Figure 18: Weighted tie strength between key (groups of) organisations in Case 2

The organisations attracting the highest number of receiving relationships can be identified by the high values associated with the direction of knowledge transfer, for example universities, the CRI and regional councils. Universities received requests for relationships (and therefore knowledge transfer) from the CRI (40), central government (43) and Private Business 1 (37). The CRI received requests from universities (25), Private Business 1 (36) and central government (38). Finally, regional councils received most requests for knowledge transfer from central 
government (35) and Private Business 1 (31). These high numbers of relationship requests reflect the existence of a high level of domain knowledge within universities, the CRI and regional councils. Due to the low number of study participants from regional councils in Case 2, there was a low number of initiating relationships from regional councils.

The organisations initiating the highest number of relationships were central government and Private Business 1 as well as universities. In particular, central government requested knowledge transfer at a high level from universities (43), regional councils (35) and CRIs (38). This may be the result of collecting knowledge from diverse organisations in order to develop guidelines or policies for the sector.

Two additional key aspects were recognised when looking at the direction of knowledge transfer (initiating and receiving relationships) in combination with the level of tie strength: the key role of central government as well as the strong relationship between universities and the CRI. The high number of initiating and receiving relationships from central government and its receiving relationships may reflect its role as a knowledge broker in the field.

The CRI indicated strong receiving relationships from universities (25). In contrast, the CRI was also highly active in initiating relationships with university study participants (40). This reflects a strong relationship with a high reciprocity: both organisational types were committed to this relationship which may reflect the traditional habits of informal knowledge transfer among CRI employees and postgraduate students.

In summary, the high tie strength among these key (types of) organisations indicated that there was a large number of informal relationships present. This result may also be underpinned by the non-existence of consultants either in the stronger or weaker relationships in Case 2. 
The strength of some of these relationships may also be due to the fact that the respective organisations involved were located in the same region. This may particularly apply to the CRI organisation and study participants from one specific university. Weaker relationships (lower contact frequency and/or number of study participants) were identified between regional councils and universities as well as regional councils and the CRI.

Besides the analysis of these key (groups of) organisations, all participating organisations were aggregated at a level reflecting the group of organisation as described earlier in this chapter. Figure 19 depicts tie strength between the seven groups of organisations (including others). In particular, Figure 19 represents study participants from CRIs, private business, universities, local government, central government, NPOs and other organisations. In comparison to Figure 18, Figure 19 shows all private businesses at an aggregated level as well as a new group of organisation: the NPO. The category for others contains either anonymous study participants or organisations that did not fit into one of the other categories. 


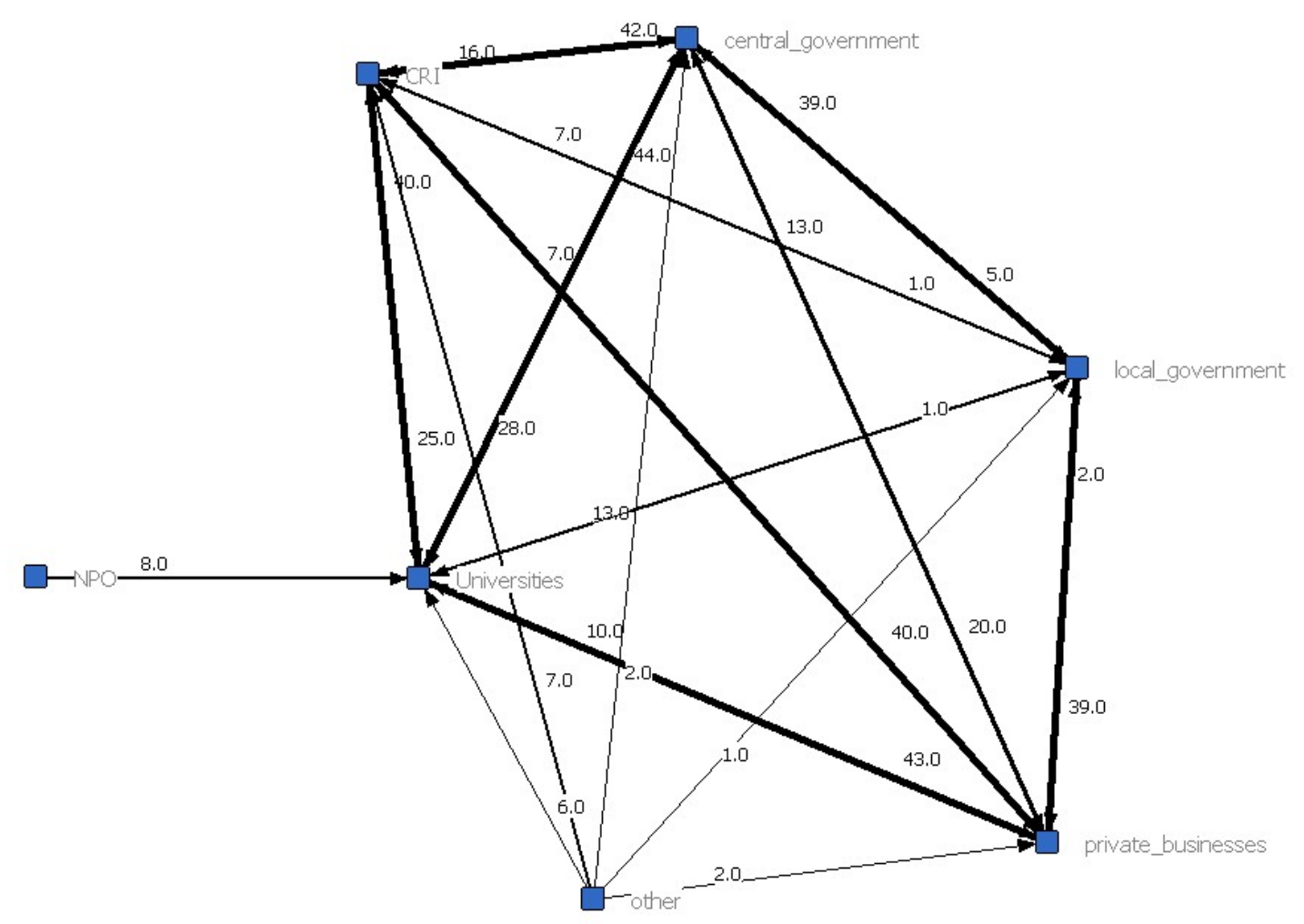

Figure 19: Tie strength between groups of organisations in Case 2

The results in Figure 19 relating to tie strength draw strongly on the results among the key organisations as shown in Figure 18. However, due to the aggregation at the group of organisational level, the level of requesting relationships and knowledge transfer from central and local government increased.

These high levels of requests $(39 ; 20)$ showed that private businesses were very interested in, for example, guidelines provided by these government agencies. However, these high levels of requests could have also been due to a close collaboration between the two groups of organisation in the marine biology sector.

In Case 2, knowledge was transferred among organisations on a 3- to 6-monthly basis. However, a few connections indicated a higher contact frequency. For example, private businesses gave monthly advice to CRIs. On the other hand, private business and CRIs sought monthly advice from universities. As the tie strength also considered 
the number of study participants per organisation, these specific contact frequencies may not be reflected by the illustrated tie strength values.

Although the NPO looks somewhat disconnected, it had a strong relationship with the universities group. One NPO participant indicated a high contact frequency for giving and seeking advice activities with universities. This relationship could not be confirmed by any university participant, as the NPO was not part of the initial set of key organisations used for the SNA data collection.

In summary, this analysis revealed not only different levels of tie strength and directions of knowledge transfer among participating organisations, but also important results on the aggregated level representing groups of organisations in the New Zealand marine biology sector, for example the important roles of central government, CRIs and universities. This finding could also be confirmed by the level of interaction analysis for Case 2 in the previous section. It also revealed universities and CRIs as the groups that were mostly contacted for knowledge transfer.

The next section reports on the centrality measures undertaken with Case 2 data.

\subsubsection{Centrality aspects}

This section focuses on appropriate centrality measurements for the network in Case 2. The most important issues to consider for undertaking these centrality measurements are outlined. This briefly repeats and also extends the descriptions and recommendations given for Case 1 in Chapter 4. The descriptions imply a significant difference between centrality measurements most suitable for explicit electronically available knowledge and tacit knowledge compared to explicit electronically unavailable knowledge. This particularly extends the description given in Chapter 4 for the tacit knowledge dimension that was added in Case 2. The centrality measurements were conducted at the type-of-organisation level. Supporting data for 
all tables showing centrality measurement scores in this section are found in Appendix 13.

Due to the variety of available centrality measures, which make different assumptions about the way in which knowledge flows in a network, it is strongly recommended to match a specific type of network flow (context-specific) to the appropriate centrality measurement. According to Borgatti (2005), the kind of trajectory that traffic may follow (geodesics, trails, paths or walks) and the method of spread (broadcast, serial replication, or transfer) are significant factors to consider. These aspects are outlined in the following section in regard to the study context of Case 2.

As this study has asked study participants for peers with whom they exchanged knowledge in the form of advice giving and seeking activities, the shortest path seems to be the predominant option for the first dimension. However, the case of transferring meta-knowledge, such as contact details of experts to peers at the workplace, enhances the geodesic concept in terms of additional paths that need to be considered. In addition, the researcher suggests that knowledge in an advice network typically does not pass the same link twice, but it may pass the same actor multiple times (trail).

Most Case 2 study participants used media such as email and face-to-face meetings to support peer-to-peer knowledge exchange. But these media could have also supported group communication, for example group meetings or when using email distribution lists. This indicates that advice giving and seeking might but not only take place in a one-to-one scenario (serial replication), but also in a one-to-many scenario (parallel replication) dependent on the medium and how it is used.

In contrast to a replication, a transferred object does not remain with the sender like used goods, money or a package (Borgatti, 2005). Knowledge could be transferred in the form of a book or a paper-based document. This reveals that this method of spread is dependent on the type of knowledge. Three types of knowledge were of 
particular interest to this study: explicit electronically available knowledge, tacit knowledge, and explicit electronically unavailable knowledge. As explicit electronically available and unavailable knowledge have already been described in Case 1 (Chapter 4), only the tacit knowledge type is explained in relation to this study's context in the following paragraph.

Tacit knowledge was the second most important type of knowledge for study participants in the advice network of Case 2. In particular, informal how-to and informal factual knowledge were important to knowledge givers in weak and strong relationships as well as knowledge seekers in strong relationships. Tacit knowledge also spreads by replication rather than transference. In a way, it could be compared to Borgatti's (2005) examples of gossip or attitudes. For example, knowledge givers do not lose their tacit knowledge the moment they give it to someone else.

Case 2 study participants used mostly explicit electronically available knowledge and tacit knowledge was the next most popular. Thirdly, explicit but electronically unavailable knowledge was also relevant. Table 30 summarises the characteristics of the three types of knowledge starting with the most important one.

Table 30: Types of knowledge and their flow characteristics

\begin{tabular}{|l|l|l|}
\hline Type of knowledge & Kind of trajectory & Method of spread \\
\hline $\begin{array}{l}\text { Explicit electronically } \\
\text { available knowledge }\end{array}$ & $\begin{array}{l}\text { Serial or parallel replication } \\
\text { dependent on medium }\end{array}$ & Shortest path or trail \\
\hline Tacit knowledge & $\begin{array}{l}\text { Serial or parallel replication } \\
\text { dependent on medium }\end{array}$ & Shortest path or trail \\
\hline $\begin{array}{l}\text { Explicit electronically } \\
\text { unavailable } \\
\text { knowledge }\end{array}$ & $\begin{array}{l}\text { Transfer or replication } \\
\text { (dependent on paper-based } \\
\text { copies) }\end{array}$ & Shortest path or trail \\
\hline
\end{tabular}

These results about the types of knowledge mostly transferred and the process of knowledge transfer guided the selection of appropriate centrality measures for this context of inter-organisational knowledge transfer and learning. As in Case 1, the following types of centrality measures were chosen:

- $\quad$ closeness centrality

- $\quad$ information centrality

- betweenness centrality 
As in Case 1, these three measurements were selected, as they assume shortest paths or 'trails'. In the advice network of Case 2, it was not assumed that knowledge would be transferred twice along the same connection, which is the assumption underlying 'walk-based' network flows. In this regard, two other well-known centrality measures (degree centrality and eigenvector centrality) were not further considered in this context, as they only support 'walk-based' network flows. This decision was also made in Case 1.

Closeness centrality and information centrality also imply the appropriate assumption for the kind of trajectory, namely replication (serial or parallel). In contrast to these two measures, betweenness centrality considers the transfer rather than the replication of knowledge. This refers to the third type of knowledge important to this study: explicit electronically unavailable knowledge. Flow betweenness was selected for Case 2, as it considers 'trails' rather than just shortest paths. The following paragraphs outline the measurement results. Each centrality measurement was described in more detail in Chapter 4.

\section{Closeness centrality measure}

Groups of organisation with a high closeness may benefit in terms of receiving knowledge earlier for knowledge reuse. According to the network typology, closeness centrality assumes shortest paths as well as replication and transfer of things that flow in the network. Therefore, this measure considers all three types of knowledge relevant to this study (tacit, explicit electronically available and unavailable knowledge) as long as this knowledge is transferred along the shortest path in the advice network. Table 31 shows the results of the closeness centrality measure for the different groups of organisation in Case 2. 
Table 31: Closeness centrality per group of organisation in Case 2

\begin{tabular}{|l|c|c|c|}
\hline Group of organisation & $\begin{array}{c}\text { In-closeness } \\
\text { centrality score }\end{array}$ & $\begin{array}{c}\text { Out-closeness } \\
\text { centrality score }\end{array}$ & $\begin{array}{c}\text { Total closeness } \\
\text { centrality score }\end{array}$ \\
\hline Universities & 100.00 & 33.33 & 133.33 \\
\hline CRIs & 85.71 & 33.33 & 119.04 \\
\hline Central government & 85.71 & 33.33 & 119.04 \\
\hline Private businesses & 85.71 & 33.33 & 119.04 \\
\hline Local government & 85.71 & 33.33 & 119.04 \\
\hline Others & 14.29 & 50.00 & 64.29 \\
\hline NPOs & 14.29 & 37.50 & 51.79 \\
\hline
\end{tabular}

In Table 31, universities with a score of 100.00 showed the highest centrality for incoming relationships and an average score of 33.33 for initiating relationships with other organisations. CRIs, central government, private businesses and local government had an equally high centrality score for both incoming and initiating relationships.

These results identified the universities as the domain knowledge leader for Case 2. In addition, results revealed a balanced knowledge transfer in terms of giving and seeking advice activities. Overall, universities had the highest total closeness centrality score, followed by CRIs, central government, private businesses and local government, all with equally high total scores. The NPO had the lowest total closeness centrality score. It therefore was the group of organisations in the least favoured position in the network in regard to receiving knowledge early. Universities were the group of organisation best connected in terms of receiving important knowledge at an early stage.

As already outlined in Chapter 4, social relationships among organisations were characterised by their different levels of tie strength, and closeness centrality binarises these valued data which is considered a disadvantage. Considering valued ties, the information centrality measure promises more accurate results of centrality in knowledge networks. 


\section{Information centrality measure}

Information centrality takes the characteristics of information/knowledge into account which includes the fact that knowledge does not necessarily follow the shortest path in a network. In addition, information centrality takes into account the actual strength of ties between organisations (it does not binarise/dichotomise data). Table 32 depicts the information centrality measures for the groups of organisation in the Case 2 advice network

Table 32: Case 2 Information centrality per group of organisation

\begin{tabular}{|l|c|}
\hline Group of organisation & Actor Information Centrality \\
\hline Universities & 30.13 \\
\hline Central government & 28.61 \\
\hline CRIs & 28.54 \\
\hline Private businesses & 28.53 \\
\hline Local government & 26.32 \\
\hline Others & 14.27 \\
\hline NPOs & 8.16 \\
\hline
\end{tabular}

Universities again showed the highest ranked centrality score, whereas the NPO had the lowest one. Compared to the results of closeness centrality, one main difference could be identified: local government agencies were less central than central government, private businesses and CRIs. The latter three groups of organisation were again almost equally central. Overall, the results of information centrality confirmed the ranked list of the most central groups of organisation resulting from the closeness centrality measurement. This is again an unexpected result, as closeness centrality does not consider different levels of tie strength compared to information centrality.

Closeness centrality and information centrality assume that knowledge is replicable in the form of explicit electronically available or tacit knowledge. As a third type of knowledge (explicit electronically unavailable) was important to several study participants in Case 2, the betweenness centrality analysis was conducted. In 
particular, flow betweenness was employed which assumes the transfer of knowledge as well as 'trails' in a network.

\section{Flow betweenness centrality}

In this study, actors might have used all of the pathways connecting them ('trails'), rather than just the shortest paths (geodesic paths). Accordingly, flow betweenness (Freeman et al., 1991) does not assume shortest paths only, but does assume that no actor is visited more than once ('trails'). As a result, CRIs had the highest score (10.88), universities were second (9.42) and central government third (5.71). These groups of organisation were followed by private businesses and local government. Other organisations and the NPO were the least central ones.

Table 33: Flow betweenness centrality scores per group of organisation in Case 2

\begin{tabular}{|l|c|}
\hline Group of organisation & Flow betweenness \\
\hline CRIs & 10.88 \\
\hline Universities & 9.42 \\
\hline Central government & 5.71 \\
\hline Private businesses & 3.39 \\
\hline Local government & 1.14 \\
\hline Others & 0 \\
\hline NPOs & 0 \\
\hline
\end{tabular}

Being based on binarised data, flow betweenness results may be most comparable to the closeness centrality results (which are also based on binarised data, i.e. they do not consider different levels of tie strength) for identifying the most central groups of organisation in the network.

In summary, these three centrality measurements appeared varyingly suitable according to the specific types of knowledge transferred in the network of Case 2. For explicit electronically available knowledge and tacit knowledge, closeness and information centrality were the most appropriate measures. These measures identified 
the most influential group of organisation, which was the universities group. Thus, universities were the group of organisation that received new or reusable knowledge earliest. This could be a competitive advantage when applying for research funds. For explicit electronically unavailable knowledge, such as books and documents however, flow betweenness centrality was employed as the most appropriate measure. Flow betweenness revealed the CRIs as the most influential group of organisation. The group of CRIs therefore was the most powerful group of organisation for controlling knowledge transfer in the network. This might be a favourable position from which to transfer knowledge in the form of learning initiatives (which could include e-learning activities).

However, as electronically available and tacit knowledge were the two most important types of knowledge to study participants in Case 2, universities were overall in a slightly more powerful position in the network than the CRIs.

In regard to the most appropriate centrality measure for this context, the researcher suggests information centrality, as it considers replicable knowledge, which was the most important type. The information centrality measurement also considered different levels of tie strength among the groups of organisation. This recommendation supports the Case 1 results described in Chapter 4.

As in Case 1, the number of study participants per group of organisation appears to have influenced the final ranking in Case 2. Thus a lower number of study participants appeared to lead to a lower centrality score (e.g. local government, NPOs).

In addition, only one CRI was the provider of professional workshops/seminars in the domain of marine biology. This might have influenced their highly ranked position in the centrality measures, especially because underlying data were partly collected from these workshop attendees. 


\subsection{Conclusion and discussion}

\section{Summary}

This chapter described and interpreted the results from Case 2. Firstly, the participating key (types of) organisations were described. Then, these (groups of) organisations were aggregated into CRIs, central government, local government (including regional councils), universities, private businesses, not-for-profit organisations (NPOs) and others (anonymous study participants or organisations that did not fit into one of the other categories). Twenty-two organisations that were active in the professional field of marine biology in New Zealand took part in Case 2.

Key findings were related to the direction of knowledge transfer, in particular the levels of interaction and the strength of relationships. Two key aspects were recognised by looking at the direction of knowledge transfer (initiating and receiving relationships) in combination with the level of tie strength: the key role of central government as well as the strong relationship between universities and the CRI.

The level of interaction in the inter-organisational knowledge transfer network of Case 2 has been investigated using univariate statistics. Sources and sinks of relationships were identified. Key sinks were

- universities

- CRIs

- local government

Central government study participants were also contacted often for knowledge transfer. The organisation groups that initiated the most relationships (key sources) were:

- private businesses

- central government 
In addition, universities and CRIs were active in initiating relationships for knowledge transfer.

In addition, the types of knowledge transferred were considered. Two different types of knowledge were the most important for Case 2 study participants:

- explicit electronically accessible knowledge

- electronically inaccessible (tacit) knowledge

Explicit electronically accessible knowledge included formal factual and how-to knowledge and meta-knowledge. Electronically inaccessible and tacit knowledge included how-to and factual knowledge.

A third type, the explicit electronically inaccessible (but codified) knowledge, was also relevant.

Structural aspects regarding the centrality of organisations were explored in depth, extending investigations carried out in Case 1. The contribution to knowledge made in relation to centrality in knowledge networks in Chapter 4 was extended for the tacit dimension of knowledge. The most suitable SNA centrality measures were identified for these different types of knowledge transfer in a network.

The researcher has again suggested information centrality as the most suitable measure, as it considers replicable knowledge, which was the most important type in Case 2. The information centrality measurement also considers different levels of tie strength among the groups of organisations. This contribution to SNA and knowledge management literature helped gain a better understanding of inter-organisational knowledge transfer structures using SNA which was a major goal of this study. 
In line with these results, this chapter helped answer the first two sub-questions of this research in regard to Case 2 results:

1) What are the key structural properties of (ties in) advice networks and their implications for knowledge transfer?

2) Which types of knowledge are transferred in the advice networks?

The key results of this case are briefly discussed in the following paragraphs.

\section{Discussion}

Study results

In Case 2, tie strength and centrality measures as well as the analysis of the levels of interaction identified a number of key players: universities, central government and CRIs as well as private businesses and local government. Key sinks were universities, CRIs and local government. Key sources were private businesses and central government. These findings may be a significant source of managerial implications. As already outlined in the Case 1 results, social network analysis results have previously been employed in the organisational context (e.g. Cross et al., 2006; Liebowitz, 2005). The knowledge about key players and their characteristics may be used to inform future business decisions.

Study participants in Case 2 did not have any opportunity to connect via a domainspecific online platform to share their knowledge and experiences (as in Case 1). Again, in the context of networking benefits and the reuse of knowledge, ICTsupported collaboration may enhance current face-to-face workshops, as collaborative ICT facilitates the creation of new relationships (Haythornthwaite, 2005) and helps 
maintain existing ones. Moreover, knowledge can be saved in one specific place and people can search and reuse this knowledge.

In addition, different levels of tie strength were related to the types of knowledge transferred in the network. Results showed that only the electronically unavailable/tacit informal how-to knowledge type, such as experiences, varied between strong and weak ties. Experiences were more often transferred in strong relationships than weak ones. Although this difference was only the case for advice seeking activities in the network, this study showed that such preferences confirm Hansen's (1999) findings regarding the transfer of different types of knowledge in weak and strong ties. Moreover, the direction of knowledge transfer (giving/seeking advice) appears important.

A contribution to knowledge has been made in relation to centrality in knowledge networks. The most appropriate SNA centrality measures were identified for different types of knowledge transfer in a network considering the two dimensions: ways that traffic (knowledge) may follow and the method of spread. This applies current literature discussing these issues (Borgatti, 2005). The selection of the most suitable centrality measures was guided by the types of knowledge most frequently used for knowledge transfer by study participants in Case 2 . These were explicit electronically accessible knowledge and electronically inaccessible (tacit) knowledge. In addition,

the explicit electronically inaccessible (but codified) type was also relevant. In this regard, information centrality was again chosen as the most suitable measure, as it considers replicable knowledge, which was the most important type in Case 2.

\section{Possible managerial implications}

Key findings of Case 2 revealed the significant role of central government and universities with relatively high levels of both initiating and receiving relationships for knowledge transfer. In addition, local government was additionally identified as a leader in this knowledge domain (key sink), besides CRIs and universities. In terms of expert knowledge, however, universities and CRIs appeared to lead among these 
groups of organisations. The influential role of central government in this knowledge domain may indicate relatively policy-led research activities in the field of marine biology research in New Zealand. However, central government also appears to be very interested in maintaining relationships (due to their high level of initiating relationships) with other organisation groups. This may indicate a collaborative development of guidelines and policies.

As Case 2 identified a strong relationship between universities and the CRIs, future trainers giving workshops and seminars could come from both groups. At the time of this research, a CRI was the only provider of professional workshops in the domain of marine biology. In addition, as a managerial implication in the context of this research, central government could organise workshops to foster discussions about guidelines and policies and maybe facilitate collaboration and joint development of them. As private businesses were key sources and thus very interested in receiving new knowledge (e.g. policies), they might be particularly interested in this option.

While this chapter has presented, interpreted and discussed the Case 2 results, the next chapter depicts the findings of Case 3. 


\section{Case 3 results}

This chapter reports on key results of Case 3 as part of this multiple case study. Firstly, it outlines the study participants' characteristics. This includes possible relationships between groups of organisations. Then, the types of knowledge transferred between study participants are outlined. Next, key findings in regard to structural properties of this social network are described. This includes tie strength and the levels of interaction. In addition, centrality measures to identify the most influential organisations in the network are described. Finally, this chapter concludes with a summary and brief discussion of the key results.

\subsection{Study participants characteristics}

This section outlines the characteristics of survey participants in Case 3. Fifty-two study participants from 30 organisations that were active in the professional field of coastal hazards in New Zealand took part in Case 3. The coastal hazards topic was taken as a boundary for a social network of people working at diverse organisations and sharing the same work interests.

Five key groups of organisation in the NZ environmental R\&D sector could be identified prior to the SNA data collection: CRIs, local government (including regional, district and city councils), private businesses, universities and central government. As in Cases 1 and 2, these groups of organisations could again be distinguished by their type of ownership (public/private) and their core business activities. An additional group of 'others' was included. This group included only one study participant from an overseas company. Although this study focussed on the NZ context, this participant was included due to the close relationships to key NZ organisations.

All participating organisations were at least partly active in the same professional field (coastal hazards). Some key organisations were more specialised in coastal hazards than others. Individual study participants had various roles in the field 
ranging from coastal planner and scientist to natural hazards analyst and policy analysts as well as lecturers. However, the job positions that were stated predominantly were scientists and analysts in the field.

Overall, the key groups of organisation participating in Case 3 were the same as in Case 2. Inter-organisational knowledge transfer between these groups of organisation in the field of coastal hazards also appeared to happen either in formal business relationships, in publicly offered seminars, workshops and conferences, or in close social relationships. Workshops and seminars that were offered to the public were held by only the group of CRIs.

Overall, a higher number of men (53.8\%) than women (46.2\%) participated in Case 3. Supporting data can be found in Appendix 15. However, there were more inexperienced female workers than males. Overall, 19 participants who answered the associated question were job beginners.

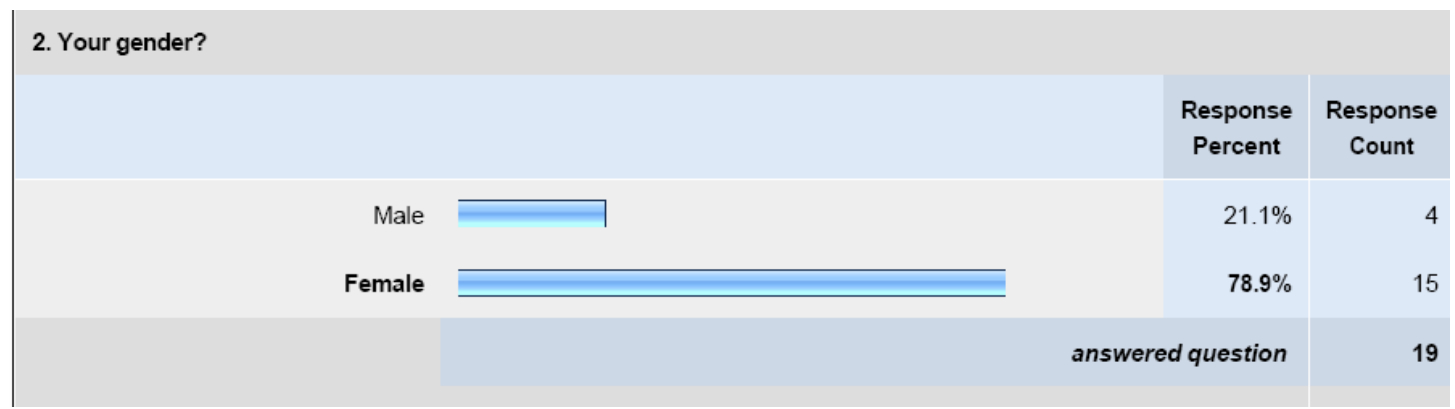

Figure 20: Job beginners (0-3 years) by gender in Case 3

Overall, a relatively high number of participants with 10 or fewer years of job experience participated as shown in the following table. However, a considerable number of experienced experts also participated in this study. 
Table 34: Study participants by job experience in Case 3

\begin{tabular}{|c|c|}
\hline & \\
\hline Answer Options & Response Percent \\
\hline$<1$ year & $7.7 \%$ \\
\hline $1-3$ years & $28.8 \%$ \\
\hline $4-10$ years & $26.9 \%$ \\
\hline $11-19$ years & $17.3 \%$ \\
\hline $20-30$ years & $15.4 \%$ \\
\hline$>30$ years & $3.8 \%$ \\
\hline
\end{tabular}

Bearing in mind the membership figures provided by the professional societies contacted for data collection, the overall response rate could be calculated at around 30 percent. However, as in Cases 1 and 2, due to a pre-selection of certain (groups of) organisations for data collection and limited control over the actual organisational affiliation of participants, this response rate remains an approximate figure.

In terms of contact frequency, inter-organisational knowledge transfer happened mostly with a 3- to 6-monthly frequency. Intra-organisational knowledge transfer always showed a higher frequency than inter-organisational knowledge transfer. Seeking and giving advice were often equally important for study participants within their organisation and also in the inter-organisational context.

Not all 52 participants answered each question. However, a majority of 47 participants answered the two survey questions on contact frequencies. As in Cases 1 and 2, participants opted out of the survey more often in the second than in the first part. This may lead to the conclusion that they considered the survey was too long. However, no particular non-respondent bias could be determined.

Table 35 depicts the number of study participants who answered the survey's questions on the frequencies of advice giving and seeking activities. Table 35 shows the number of study participants from each key organisational type. 
Table 35: No of study participants per organisational key type in Case 3

\begin{tabular}{|l|c|}
\hline Group of organisation & No. of study participants \\
\hline Local government & 15 \\
\hline Private businesses & 10 \\
\hline CRIs & 8 \\
\hline Central government & 8 \\
\hline Universities & 5 \\
\hline Others & 1 \\
\hline All organisations & 47 \\
\hline
\end{tabular}

Besides the low number of study participants in the 'others' group, an almost equally distributed number of people from diverse groups of organisation participated. Private businesses and local government agencies had the highest number of responses. In addition, there was a higher number of responses from local government and private businesses than from CRIs, central government and universities. These three groups of organisations with the lower participation numbers also had lower member numbers in the professional society that was contacted for survey distribution in Case 3.

The following section introduces the possible relationships between these study participants described.

The relationships between different groups of organisation

This section describes the relational characteristics among the key groups of organisations. As all organisations belonged to the same professional field (coastal hazards), one would expect a number of formal business relationships such as alliances as well as various informal relationships, for example through friendships or acquaintanceships. In Case 3, the co-existence of and also dynamics between formal and informal relationships was confirmed by study participants in the follow-up questions to the online survey (Phase 3):

"There is a certain amount of informal working together such as regional councils assisting with CRI research (supplying general info, supplying hazard event info, 
facilitating relationships, providing a steer, providing local

knowledge etc). Personally, I try to keep the CRIs interested in our region.

Sometimes the relationship can lead to the CRI undertaking research with

funding coming from other than regional councils e.g. the Earthquake

Commission" (Participant 1)

"Formally, we have regular cross agency meetings on particular topics. Informally we network with individuals from other agencies at conferences and seminars." (Participant 2)

"We have formal contracts with CRIs, central and regional/local government and businesses, and are working together on pre-determined government research and commercial projects. We also have informal relationships with all of the above parties to work on other research projects (e.g. we might be working in an organisation's 'patch' so we get informal agreement to work there)." (Participant 3)

In regard to this study's goals, inter-organisational knowledge transfer and learning were seen as a significant national topic as stated by a participant from central government: "The Ministry for the Environment is responsible for overseeing Resource Management Act issues and therefore also has a vested interested in encouraging joint learning opportunities." (Participant 4)

Case 3 revealed the following possible relationships between participating key groups of organisations: 
Table 36: Possible relationships between groups of organisation in Case 3

\begin{tabular}{|c|c|}
\hline $\begin{array}{ll}> & \text { CRIs - local government } \\
> & \text { CRIs - private businesses } \\
> & \text { CRIs - central government } \\
> & \text { CRIs - universities } \\
> & \text { CRIs - others }\end{array}$ & $\begin{array}{l}>\text { Private business }- \text { local government } \\
>\quad \text { Private business }- \text { others }\end{array}$ \\
\hline $\begin{array}{l}>\text { Universities - local government } \\
>\text { Universities - private businesses } \\
>\text { Universities - central government } \\
>\text { Universities - others }\end{array}$ & $>$ Local government - others \\
\hline $\begin{array}{l}>\text { Central government }- \text { local government } \\
>\quad \text { Central government }- \text { private } \\
\text { businesses } \\
>\quad \text { Central government }- \text { others }\end{array}$ & \\
\hline
\end{tabular}

These possible inter-organisational relationships represented 15 different kinds of relationships. Moreover, when considering the diverse contact frequencies (once every week, once every month, once every three months, once every six months, or never), the variety of relationships multiplied.

The following section describes the types of knowledge transferred in the social relationships of Case 3.

\subsection{Knowledge characteristics}

Knowledge characteristics were an important factor in relation to inter-organisational knowledge transfer in the advice network of Case 3. This section outlines the more important types of knowledge transferred. The importance of various types of knowledge to different types of social relationships is explained. The description of the types of knowledge in this case can be found in Chapters 4.2 and 5.2.

The types of knowledge that were most important to study participants are shown in Table 37. They could be categorised as: formal factual knowledge (electronically accessible), meta-knowledge (particularly references to other sources of knowledge, 
electronically accessible) and informal knowledge (factual and how-to, both partly electronically accessible). However, the most important electronically inaccessible types were mostly informal factual and how-to knowledge as well as meta-knowledge to some degree.

Specific types of knowledge were transferred in different kinds of relationships with different tie strengths and knowledge transfer directions. Table 37 summarises the key results for giving and seeking advice activities in strong and weak social relationships between organisations. Forty-five study participants stated the following types of knowledge were significant for their inter-organisational knowledge transfer. Electronically inaccessible knowledge is highlighted in bold. Supporting data can be found in Appendix 16.

Table 37: Types of knowledge transferred in Case 3

\begin{tabular}{|l|c|c|l|l|l|l|}
\hline & $\begin{array}{l}\text { Electron. } \\
\text { accessible } \\
\text { formal } \\
\text { documents } \\
\text { (facts and/or } \\
\text { how-to) }\end{array}$ & $\begin{array}{l}\text { Electron. } \\
\text { accessible } \\
\text { meta- } \\
\text { knowledge }\end{array}$ & $\begin{array}{l}\text { Electron. } \\
\text { accessible } \\
\text { informal } \\
\text { factual } \\
\text { and/or how } \\
\text { to } \\
\text { knowledge }\end{array}$ & $\begin{array}{l}\text { Electron. } \\
\text { inaccessible, } \\
\text { lnon-codified } \\
\text { informal, } \\
\text { factual } \\
\text { and/or how- } \\
\text { to knowledge }\end{array}$ & $\begin{array}{l}\text { Electron. } \\
\text { inaccessible } \\
\text { meta- } \\
\text { knowledge }\end{array}$ & $\begin{array}{l}\text { Electron. } \\
\text { inaccessible, } \\
\text { codified, } \\
\text { informal } \\
\text { factual } \\
\text { and/or how- } \\
\text { to knowledge }\end{array}$ \\
\hline $\begin{array}{l}\text { Giving } \\
\text { strong } \\
\text { ties }\end{array}$ & $\mathrm{X}$ & $\mathrm{X}$ & $\mathrm{X}$ & $\mathbf{X}$ & & \\
\hline $\begin{array}{l}\text { Giving } \\
\text { weak ties }\end{array}$ & $\mathrm{X}$ & $\mathrm{X}$ & $\mathrm{X}$ & & $\mathbf{X}$ & $\mathbf{X}$ \\
\hline $\begin{array}{l}\text { Seeking } \\
\text { strong } \\
\text { ties }\end{array}$ & $\mathrm{X}$ & $\mathrm{X}$ & & & $\mathbf{X}$ & \\
\hline $\begin{array}{l}\text { Seeking } \\
\text { weak ties }\end{array}$ & $\mathrm{X}$ & $\mathrm{X}$ & $\mathrm{X}$ & & & \\
\hline
\end{tabular}

In Table 37, key results indicated that study participants in strong relationships often transferred the same types of knowledge as study participants in weaker relationships. However, weak ties additionally transferred electronically inaccessible metaknowledge and electronically inaccessible, but codified, informal factual (e.g. datasets) and/or how-to knowledge (e.g. procedural knowledge). In contrast to weak ties, strong ties also transferred electronically inaccessible/non-codified informal factual and/or how-to knowledge. As these two types of knowledge appeared more complex 
than meta-knowledge or codified informal knowledge, this might have been an indicator of an easier transfer of complex knowledge in strong relationships. In regard to giving and seeking advice activities, no significant differences could be recognised.

In summary, six different types of knowledge, as illustrated in Table 37, were significant for inter-organisational knowledge transfer in Case 3. Three of these six types were electronically inaccessible: electronically inaccessible/non-codified informal factual (e.g. research data) and/or how-to knowledge (e.g. experiences), electronically inaccessible meta-knowledge and electronically inaccessible but codified informal factual (e.g. data-sets) and/or how- to knowledge (e.g. procedural knowledge). Electronically inaccessible knowledge in the form of non-codified or tacit knowledge was the most important type for study participants. Examples of electronically available knowledge were pdf documents, websites and emails (Phase 3). Tacit knowledge was transferred for example in a regionally based interest group. Thus, regional proximity among key organisations, such as CRIs and local governments, facilitated the transfer of tacit knowledge enormously. Results showed that the types of knowledge varied between weak and strong ties, but not significantly between givers and seekers of knowledge.

The following section reports key findings related to structural properties of the advice network in which the described key (groups of) organisations have participated. 


\subsection{Key findings related to structural properties of the advice network}

Key findings of this section report on the levels of interaction in the advice network and tie strength. As in Cases 1 and 2, appropriate centrality measures were selected to analyse the most central organisations (groups of organisations) for knowledge transfer in the social network of Case 3. Finally, this chapter is summarised and findings are discussed.

\subsubsection{The levels of interaction}

As for Cases 1 and 2, advice giving to peers and advice seeking from peers at the workplace were chosen as the activities that define the direction of knowledge transfer in Case 3. This transfer can be uni- or bi-directional between sender and receiver.

Descriptive statistics of network data describe the distribution of relational characteristics, such as the level of interaction, among actors. Table 38 consists of descriptive statistics presenting interactions (i) among all groups of organisation in the social network of Case 3 during the last year. These descriptive statistics were derived from the information centrality measure, as this measure was considered the most appropriate. Results are based on valued data. However, the network centralisation index was again derived (as in Case 1 and Case 2) from flow betweenness centrality, as closeness and information centrality did not provide this information. The data described below indicate a considerable variation in activity levels expressed by a medium variance measure (26.38). Data used for these measurements are found in Appendix 17. 
Table 38: Descriptive statistics of the Case 3 network

\begin{tabular}{|c|c|}
\hline $\begin{array}{l}\text { Descriptive statistics for each measure, } \\
\text { Summary statistics: Tie (i) }\end{array}$ & \\
\hline Mean & 16.09 \\
\hline Std. Dev. & 5.13 \\
\hline $\begin{array}{l}\text { Coefficient of variation (standard } \\
\text { deviation / mean } \times 100 \text { ) }\end{array}$ & 31.88 \\
\hline Variance & 26.38 \\
\hline Minimum & 4.65 \\
\hline Maximum & 19.12 \\
\hline Network centralisation index & $19.80 \%$ \\
\hline
\end{tabular}

Interactions in this network included a low number of low level interactions (weak ties) and five key groups of organisation with a higher level of interaction indicating strong ties. These five key groups of organisation were universities, CRIs, central government, local government and private businesses. These types, in particular local government, CRIs and private businesses, might represent the preferred sources for domain knowledge in the professional sector of coastal hazards.

The network centralisation index $(19.80 \%)$ of this social network was relatively low compared to a pure star network with an index of $100 \%$ (Hanneman, 2005). The low network centralisation index here shows that there was no single organisation in the network that controlled the network by itself. This indicated a decentralised interorganisational network.

In order to present more precise results, as in Case 1 and 2, Tables 37 and 38 depict univariate statistics for the groups of organisation. A summary of descriptive statistics for sending and receiving activities provides a better insight into the network's activities. These activities included initiating relationships for knowledge transfer or receiving requests for relationships and knowledge transfer. In this regard, key sources and key sinks could be identified. These differences among groups of organisations' immediate connections may be critical in explaining how they view the remaining network, and how the rest of the network views them. Table 39 depicts the univariate statistics per group of organisation for receiving relationship requests. 
Table 39: Summary of univariate statistics for groups of organisations in Case 3 by column (key sinks)

\begin{tabular}{|l|c|c|c|c|c|c|}
\hline & CRIs & Universities & $\begin{array}{l}\text { Private } \\
\text { businesses }\end{array}$ & $\begin{array}{l}\text { Central } \\
\text { government }\end{array}$ & $\begin{array}{l}\text { Local } \\
\text { government }\end{array}$ & Others \\
\hline Mean & $\mathbf{1 1 . 8 0}$ & $\mathbf{1 3 . 0 0}$ & $\mathbf{1 5 . 2 0}$ & $\mathbf{2 0 . 2 0}$ & $\mathbf{1 9 . 2 0}$ & $\mathbf{0 . 0 0}$ \\
\hline Std. Dev. & 8.79 & 8.15 & 11.75 & 12.60 & 10.06 & 0.00 \\
\hline Variance & 77.36 & 66.40 & 138.16 & 158.96 & 101.36 & 0.00 \\
\hline Minimum & 1.00 & 1.00 & 0.00 & 0.00 & 2.00 & 0.00 \\
\hline Maximum & 26.00 & 22.00 & 35.00 & 36.00 & 30.00 & 0.00 \\
\hline
\end{tabular}

This presentation of the data revealed a pattern of sink behaviour (Hanneman 2005, p.43) represented by the fact that some groups of organisation in the network were contacted for knowledge transfer more often than others. In this sense, a higher mean value indicates a type that received a higher number of requests for relationships (formal or informal) than other groups of organisation (Hanneman, 2005). The key sinks were central government, local government and private businesses. University and CRI study participants were also contacted often for knowledge transfer.

The groups of organisation that were 'sources' of relationships rather than 'sinks' are shown in Table 40. The groups of organisation that initiated the most relationships (key sources) were local government and CRIs. With a mean value of 17.4, private businesses were also active in initiating relationships for knowledge transfer.

Table 40: Summary of univariate statistics for groups of organisations in Case 3 by row (key sources)

\begin{tabular}{|l|c|c|c|c|c|c|}
\hline & CRIs & Universities & $\begin{array}{l}\text { Private } \\
\text { businesses }\end{array}$ & $\begin{array}{l}\text { Central } \\
\text { government }\end{array}$ & $\begin{array}{l}\text { Local } \\
\text { government }\end{array}$ & Others \\
\hline Mean & $\mathbf{1 8 . 8 0}$ & $\mathbf{8 . 2}$ & $\mathbf{1 7 . 4}$ & $\mathbf{1 0 . 8}$ & $\mathbf{2 3 . 4}$ & $\mathbf{0 . 8 0}$ \\
\hline Std. Dev. & 10.07 & 5.07 & 9.97 & 8.42 & 13.11 & 0.75 \\
\hline Variance & 101.36 & 25.76 & 99.44 & 70.96 & 171.84 & 0.56 \\
\hline Minimum & 0.00 & 0.00 & 0.00 & 0.00 & 0.00 & 0.00 \\
\hline Maximum & 30.00 & 14.00 & 29.00 & 25.00 & 36.00 & 2.00 \\
\hline
\end{tabular}

Overall, local government had a high number of initiating relationships as well as a relatively high number of incoming relationships requests for knowledge transfer. Therefore, they were suggested as the knowledge broker in the field of coastal hazards. 
In SNA, linkages that show the direction of knowledge transfer can also be weighted by the level of tie strength. The following paragraphs outline how tie strength was measured in the inter-organisational context of Case 3 .

\subsubsection{Tie strength}

A whole network approach, as explained in the Research Methods chapter, was employed for collecting and visualising tie strength. Tie strength between groups of organisation were measured by contact frequency (once every week, once every month, once every three months, once every six months, or never) and the number of study participants from each type. In addition, separately collected tie strength values from giving and seeking advice activities were summarised to present a level of tie strength including all advice activities. Supporting data are found in Appendices 17 and 18 .

Figure 21 illustrates this advice network showing the key groups of organisation. This figure indicates the strength and direction of knowledge transfer within the network. Tie strength is illustrated by line thickness and the values at each line. The two values indicate the tie strength of either receiving or initiating relationships. The direction of knowledge transfer comprised giving and seeking advice activities and is illustrated by an arrow at the end of the line. 


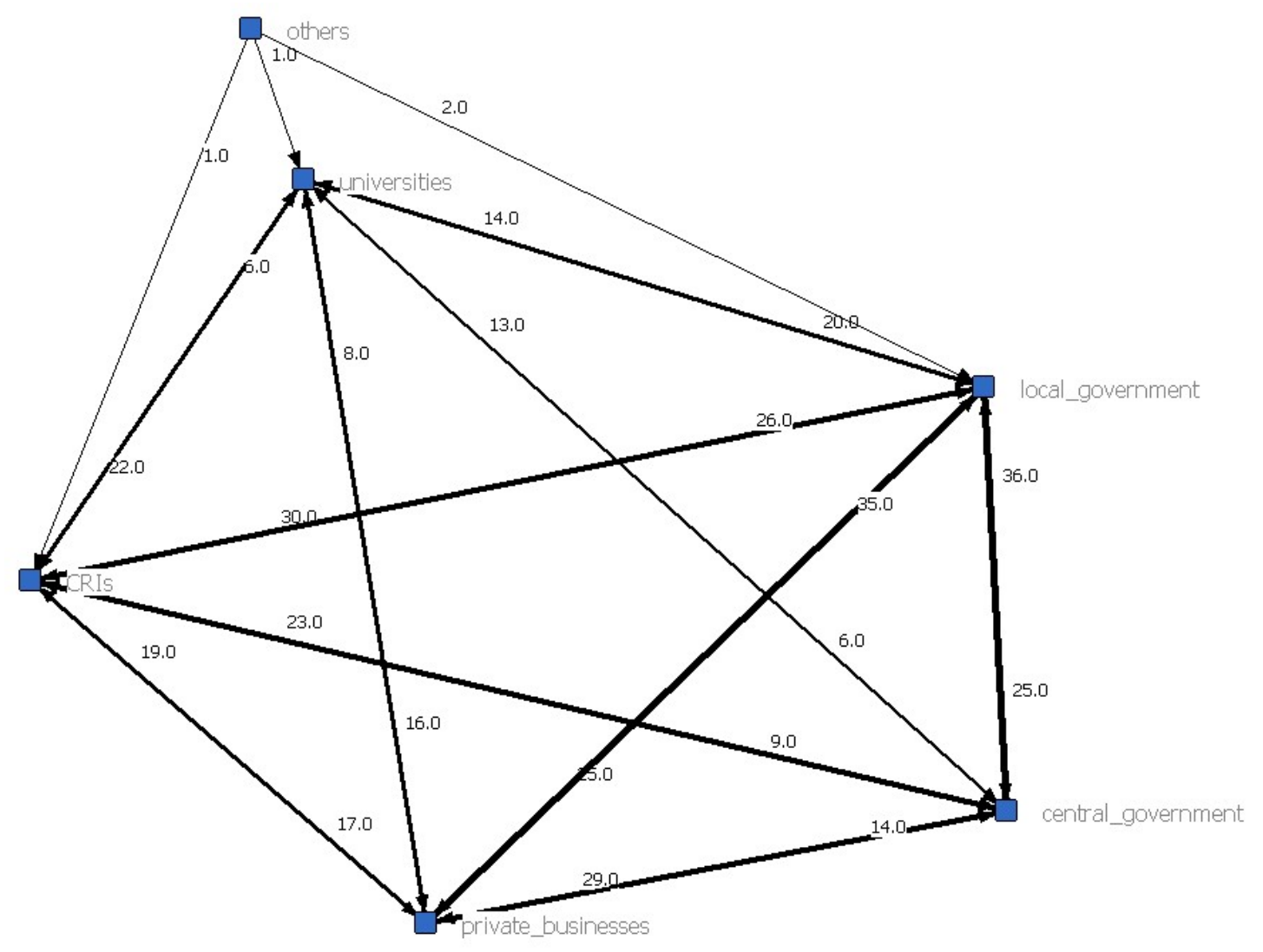

Figure 21: Case 3 advice network (whole network)

Figure 21 presents the results of measuring tie strength and illustrates the whole network of the six groups of organisation including the 'Others' category in the coastal hazards field. They either gave advice to people from other organisations in the network or sought advice from people working at other organisations during the last year. Five groups of organisation (CRIs, local government, private businesses, universities and central government) with a total number of 40 study participants were strongly connected. They can be identified by their line thickness and high tie strength values in the network. In addition, a number of weak ties (thin lines) with a total number of four groups of organisation (Others, CRIs, local government, universities) comprising seven individual study participants remained. Coded data underlying these tie strength measurements can be found in Appendix 17.

The organisations attracting the highest number of receiving relationships could be identified by the high values associated with the direction of knowledge transfer, for 
example central government, local government and private businesses. Private businesses received requests for relationships (and therefore knowledge transfer) from the CRIs (19), central government (14) and local government agencies (35). Central government agencies received requests from CRIs (23) and local government (36). Finally, local government received the highest requests for knowledge transfer from central government (25), CRIs (30) and private businesses (25). These high numbers of relationship requests reflected the existence of a high level of domain knowledge within central government, local government and private businesses.

The organisations initiating the highest number of relationships were also local government, CRIs and private businesses. In addition, universities and central government showed a fairly high level of initiating relationships. For example, central government agencies might have collected knowledge from diverse organisations in order to develop guidelines for the sector (e.g. the MfE Hazards guidelines). Universities may have wanted to initiate knowledge transfer in order to conduct research projects with industry or government. However, the key relationships in Case 3 all included local government. They were between local and central government (36 and 25), private businesses (35 and 25) and CRIs (30 and 26).

In regard to the contact frequency only, most groups of organisation transferred knowledge with a 3- to 6-monthly frequency. However, a few types gave monthly advice to another group of organisation: central government to private businesses, and private businesses to local government. In this regard, the private businesses appeared to connect central and local government.

In summary, this analysis revealed different levels of tie strength and directions of knowledge transfer among participating key groups of organisation in the New Zealand coastal hazards sector. This also included the important role of local government. The high tie strength among the key groups of organisation may indicate that there was a large number of informal relationships present. 
The following section outline the centrality measures undertaken to identify the most powerful groups of organisation in the advice network of Case 3.

\subsubsection{Centrality aspects}

This section describes appropriate centrality measurements for the network in Case 3. The most important issues to consider for undertaking these centrality measurements have already been outlined in Chapter 4 and Chapter 5. The appropriate centrality measurements should consider the different types of knowledge most important for transfer.

In Case 3, these types were explicit electronically available knowledge, tacit knowledge and explicit electronically unavailable knowledge. Study participants mostly used explicit electronically available knowledge and tacit knowledge was the next most important. Thirdly, explicit but electronically unavailable knowledge was also relevant. Table 41 summarises the characteristics of the three types of knowledge, in terms of kind of trajectory and method of spread, starting with the most important one. Supporting data for all tables showing centrality measurement scores in this section are found in Appendix 18.

Table 41: Types of knowledge and their flow characteristics

\begin{tabular}{|l|l|l|}
\hline $\begin{array}{l}\text { Type of } \\
\text { knowledge }\end{array}$ & Kind of trajectory & Method of spread \\
\hline $\begin{array}{l}\text { Explicit } \\
\text { electronically } \\
\text { available } \\
\text { knowledge }\end{array}$ & $\begin{array}{l}\text { Serial or parallel replication } \\
\text { dependent on medium }\end{array}$ & Shortest path or trail \\
\hline Tacit knowledge & $\begin{array}{l}\text { Serial or parallel replication } \\
\text { dependent on medium }\end{array}$ & Shortest path or trail \\
\hline $\begin{array}{l}\text { Explicit } \\
\text { electronically } \\
\text { unavailable } \\
\text { knowledge }\end{array}$ & $\begin{array}{l}\text { Transfer or replication } \\
\text { (dependent on paper-based } \\
\text { copies) }\end{array}$ & Shortest path or trail \\
\hline
\end{tabular}

These results about the types of knowledge most often transferred and the process of knowledge transfer guided the selection of appropriate centrality measures for this 
context of inter-organisational knowledge transfer and learning. As in Cases 1 and 2, the following types of centrality measures were chosen:

- $\quad$ closeness centrality

- $\quad$ information centrality

- $\quad$ betweenness centrality

These three measurements were selected because they assume shortest paths or 'trails'. Closeness centrality and information centrality also imply the appropriate assumption for the kind of trajectory, namely replication (serial or parallel). In contrast to these two measures, betweenness centrality considers the transfer rather than the replication of knowledge. This refers to the third type of knowledge important to this study: explicit electronically unavailable knowledge. Flow betweenness, which is a variant of betweenness centrality, was selected in particular, as it considers 'trails' rather than just shortest paths. Each centrality measurement has been described in more detail in Chapter 4.

The following paragraphs outline the measurement results.

\section{Closeness centrality measure}

Groups of organisation with a high closeness may benefit in terms of receiving knowledge earlier for knowledge reuse. According to the network typology, closeness centrality assumes shortest paths as well as replication and transfer of things that flow in the network. Therefore, this measure considers all three types of knowledge relevant to this study (tacit, explicit electronically available and unavailable knowledge) as long as this knowledge is transferred along the shortest path in the advice network. Table 42 shows the results of the closeness centrality measure for the different groups of organisation in Case 3. 
Table 42: Closeness centrality per group of organisation in Case 3

\begin{tabular}{|l|c|c|c|}
\hline Group of organisation & $\begin{array}{c}\text { In-closeness } \\
\text { centrality score }\end{array}$ & $\begin{array}{c}\text { Out-closeness } \\
\text { centrality score }\end{array}$ & $\begin{array}{c}\text { Total closeness } \\
\text { centrality score }\end{array}$ \\
\hline Universities & 100.00 & 50.00 & 150.00 \\
\hline CRIs & 100.00 & 50.00 & 150.00 \\
\hline Local government & 100.00 & 50.00 & 150.00 \\
\hline Central government & 83.33 & 50.00 & 133.33 \\
\hline Private businesses & 83.33 & 50.00 & 133.33 \\
\hline Others & 16.67 & 71.43 & 88.10 \\
\hline
\end{tabular}

Universities, CRIs and local government agencies show the highest centrality with a score of 100.00 for incoming relationships and an average score of 50.00 for initiating relationships with other organisations. Central government and private businesses had a medium and equally high closeness centrality score for both incoming and initiating relationships.

These results identified the universities, CRIs and local government as the domain knowledge leaders for Case 3. Particularly for the universities this is an unexpected result, as only few study participants came from universities compared to CRIs or local governments. However, universities, CRIs and local government were therefore the groups of organisation best connected in terms of receiving important knowledge at an early stage. 'Others' had the lowest total closeness centrality score and was therefore the group of organisation in the least favoured position in the network in regard to receiving knowledge early.

As already outlined for Cases 1 and 2, social relationships among organisations were characterised by their different levels of tie strength. However, closeness centrality binarises these valued data which is considered a disadvantage. Considering valued ties, the information centrality measure promises more accurate results of centrality in knowledge networks. 


\section{Information centrality measure}

Information centrality takes the characteristics of information/knowledge into account which includes the fact that knowledge does not necessarily follow the shortest path in a network. In addition, information centrality considers the actual strength of ties between organisations (it does not binarise/dichotomise data). Table 43 depicts the information centrality measures for the groups of organisation in the Case 3 advice network.

Table 43: Information centrality per group of organisation in Case 3

\begin{tabular}{|l|c|}
\hline Group of organisation & Actor Information Centrality \\
\hline Local government & 19.12 \\
\hline CRIs & 18.46 \\
\hline Central government & 18.31 \\
\hline Private businesses & 18.28 \\
\hline Universities & 17.76 \\
\hline Others & 4.65 \\
\hline
\end{tabular}

Local government agencies showed the highest ranked centrality score, whereas 'others' again had the lowest one. Compared to the results of closeness centrality, some differences in the ranked order could be identified: central government agencies and private businesses were more central than universities. These results may be due to the consideration of different levels of tie strength in information centrality in contrast to closeness centrality. Therefore, these information centrality results show more accurate results in terms of centrality.

Closeness centrality and information centrality assume that knowledge is replicable in the form of explicit electronically available or tacit knowledge. As a third type of knowledge (explicit electronically unavailable) was important to several study participants in Case 3, the betweenness centrality measure was also conducted. In particular, flow betweenness was employed which assumes the transfer of knowledge as well as 'trails' in a network. 
Flow betweenness centrality

In this study, actors might have used all of the pathways connecting them ('trails'), rather than just the shortest paths (geodesic paths). Accordingly, flow betweenness (Freeman et al., 1991) does not assume shortest paths only, but does assume that no actor is visited more than once ('trails'). As a result, local government agencies had the highest score (6.83), followed by CRIs (6.51) and private businesses (2.80). These groups of organisation were followed by central government and universities. Other organisations were the least central ones.

Table 44: Flow betweenness centrality scores per group of organisation in Case 3

\begin{tabular}{|l|c|}
\hline Group of organisation & Flow betweenness \\
\hline Local government & 6.83 \\
\hline CRIs & 6.51 \\
\hline Private businesses & 2.80 \\
\hline Central government & 2.60 \\
\hline Universities & 2.43 \\
\hline Others & 0 \\
\hline
\end{tabular}

Being based on binarised data, flow betweenness results may be best comparable to the closeness centrality results which are also based on binarised data. This means that they do not consider different levels of tie strength for identifying the most central groups of organisation in the network.

In summary, these three centrality measurements appeared suitable depending on the specific types of knowledge transferred in the network of Case 3. For explicit electronically available knowledge and tacit knowledge, closeness and information centrality were the most appropriate measures. These measures identified the most influential group of organisation, which was the local government group. Thus, local government agencies were the group of organisation that received new or reusable knowledge earliest. These network connections represent an important information asset that could be a competitive advantage when applying for research funds. For explicit electronically unavailable knowledge, such as books and documents however, flow betweenness centrality was employed as the most appropriate measure. Flow 
betweenness results again revealed local government as the most influential organisation group. The local government group was therefore the most powerful group of organisation for controlling knowledge transfer in the network. This might be an ideal position from which to transfer knowledge in the form of learning initiatives (which could include e-learning activities).

In regard to the most appropriate centrality measure for this context, the researcher suggests information centrality, as it considers replicable knowledge, which was the most important type. The information centrality measurement also considered different levels of tie strength among the groups of organisation. This recommendation supports Case 1 (Chapter 4) and Case 2 (Chapter 5) results.

\subsection{Conclusion and discussion}

\section{Summary}

This chapter described and interpreted the results of Case 3. Fifty-two people from 30 organisations who were active in the professional field of coastal hazards in New Zealand participated in Case 3. Key findings included the types of knowledge transferred for advice giving and seeking activities, the strength of relationships and the levels of interaction.

By investigating the direction of knowledge transfer (initiating and receiving relationships) in combination with the level of tie strength, the key role of local government agencies as knowledge brokers could be identified. Key sinks were:

- central government

- local government

- private businesses

University and CRI study participants were also contacted often for knowledge transfer. 
Key sources were:

- local government

- CRIs

In addition, private businesses and central government were also active in initiating relationships for knowledge transfer.

The types of knowledge transferred in the network were also investigated. As in Case 2, two diverse types of knowledge were significant to Case 3 study participants:

- explicit electronically accessible knowledge

- electronically inaccessible (tacit) knowledge

Explicit electronically accessible knowledge included meta-knowledge, formal factual knowledge and informal how-to knowledge. Electronically inaccessible (tacit) knowledge included meta-knowledge and informal factual knowledge.

A third type, explicit electronically inaccessible (but codified) knowledge, was also relevant.

Structural aspects regarding the centrality of organisations were illustrated and explained. Characteristics of the Case 3 network showed that there were a few, almost equally, central groups of organisations in the network: local government, CRIs and central government. In regard to the most appropriate SNA centrality measure for the different types of knowledge transferred in a network, the researcher suggested information centrality, as in Cases 1 and 2. Information centrality considers replicable knowledge (in the form of electronically accessible knowledge), which was the most important type in Case 3. The information centrality measurement also takes the different levels of tie strength among the organisation groups into account. 
In line with these results, this chapter helped answer the first two sub-questions of this research in regard to Case 3's results:

1) What are the key structural properties of (ties in) advice networks and their implications for knowledge transfer?

2) Which types of knowledge are transferred in the advice networks?

The key results of this case are briefly discussed in the following paragraphs.

\section{Discussion}

Study results

As in Cases 1 and 2, study participants did not have any opportunity to connect via an online platform to discuss issues around coastal hazards management and share their knowledge and experiences. However, a related professional society provided a newsletter online. The online platform of this society could also be used for further development to provide online collaborative tools to the public. In addition, in the context of networking benefits and the reuse of knowledge, ICT-supported collaboration may enhance current face-to-face workshops, as collaborative ICT facilitates the creation of new relationships (Haythornthwaite, 2005) and helps maintain existing ones. Moreover, knowledge can be saved in one place and people can search and reuse this knowledge.

This chapter revealed transfers of different knowledge in weak and strong ties. People in weak ties preferred to transfer electronically unavailable, but codified, informal factual knowledge, which could for example be unpublished research material. On the other hand, people in strong ties transferred electronically unavailable (non-codified) informal factual (unpublished research material) and/or tacit how-to knowledge (e.g. 
experiences). This indicated a greater transfer of non-codified and tacit knowledge in strong ties, whereas a transfer of more codified knowledge took place in weak ties. As tacit knowledge tends to be more complex in nature than codified knowledge, these results confirm Hansen's (1999) study that argues for a more effective transfer of complex knowledge in strong ties rather than weak ties.

As in Cases 1 and 2, this chapter applied SNA methods to the knowledge management context in organisations. A number of key players in the field of coastal hazards could be identified. This included the key sinks - central and local government and private businesses - and the key sources - local government and CRIs. Local government agencies had therefore a key role in initiating and receiving relationships for knowledge transfer. This role may be interpreted as a key knowledge brokering role in the domain of coastal hazards. In addition, the most central groups of organisations in the network were local government, CRIs and central government. As suggested in Cases 1 and 2, these key findings could guide future business decisions in terms of collaboration and knowledge transfer (Cross et al., 2006; Liebowitz, 2005).

\section{Possible managerial implications}

In Case 3, the very powerful role of local government departments shows a high application of research outcomes (from CRIs and possibly private businesses) and policies and guidelines (from central government) in local areas. In New Zealand, research in coastal hazards is connected strongly to climate research and also Māori related issues. Thus, it is an area of very high interest at the national level, and central government plays a key role in this domain. As central government was the most important key sink, there seems to also be a tendency towards policy-led research activities in the field of coastal hazards management in New Zealand. A related and confirming comment was made by a study participant in Phase 3 (follow-up questions) of Case 3. 
"...coastal management is unfortunately policy led, rather than scientifically led..." (Participant 3)

There also seem to be issues around the quality of research work:

"...There are reports taken as true - when the report facts are not true."

(Participant 3)

As a managerial implication, these results indicate the need for a more collaborative development of guidelines and policies and their application. Besides government departments, other groups such as private businesses, universities and also local people should be able to discuss and contribute to related issues. This external connectivity would provide balanced and appropriate sources of informal earning in the workplace.

While this chapter has presented the Case 3 results, the next chapter depicts the findings of the cross-case analysis. 


\section{Key results of the cross-case analysis: Inter- organisational knowledge transfer in small-world networks}

This chapter presents the key results of the cross-case analysis in two major parts.

The first part presents the key results in regard to the inter-organisational knowledge transfer in, and structural properties of, the three social networks. This description starts with an outline of the nature of small-world networks in general and an illustration of the three small-world social networks of this study in particular. Then, intermediate ties are defined and their important role in this study's networks is explained. In addition, key roles in these networks, including key sinks, key sources, knowledge brokers and the importance of intermediate ties, are demonstrated. Then, the most important types of knowledge transferred are described.

The second main part of this chapter presents key results of the cross-case analysis in regard to the actual media used, preferred media characteristics (in relation to MST) as well as the ICT-based knowledge services for learning (in relation to the knowledge ecology model). Key similarities and key differences among the three cases are described. Finally, this chapter concludes with a summary and discussion of the key findings.

\subsection{The small-world networks}

This section reports on the small-world network topology findings of the three social networks studied. Firstly, the small-world network structure is defined. This is followed by the description of the two main characteristics of small-world networks, namely a high number of short paths and a high degree of local clustering. As Cases 2 and 3 show similar results, Case 1 examples are used for a clear description. However, all networks are additionally illustrated in this section. Then, the relevance 
of these results in the knowledge transfer context of this study is described and the conditions for ICT support outlined.

\subsubsection{Definition}

In contrast to a centralised network, which has one single network member in the most central position and two network members are seldom directly connected, a small-world network is defined as a moderately decentralised type of network. It has a high degree of local clustering and a high number of direct connections between any two network members (Albert \& Barabasi, 2000; Kwon et al., 2007).

Decentralised small-world structures are typical in many large-scale real-world networks such as biological networks, the Internet or social networks (Nguyen, 2006). For example, a large social network of 240 million people using the same instant messaging tool (Microsoft Messenger) was identified by Leskovec and Horvitz (2008) as a small-world network. In contrast to completely random networks, small-world networks consist of a number of randomly structured relations and additional local clusters that represent some degree of centralisation (Kwon et al., 2007; Watts \& Strogatz, 1998). Local clustering facilitates reliable accessibility and links between local clusters in a small-world network can provide easy access in the network (White \& Houseman, 2003).

According to Kwon et al. (2007), a small-world network structure is a key ontological structure for Information Processing Networks (IPNs). 
This study's social networks were identified as small-world networks according to two main characteristics:

- firstly, a high number of short paths (small average path length) between any two members in the network, and

- secondly, by a high clustering coefficient that indicates the existence of a certain degree of centralisation in an overall decentralised network.

\subsubsection{A high number of short paths}

In general, a path in a network is a linkage between at least two network members and is illustrated by the lines in a network. However, no connection is repeated and no network member is visited twice (Borgatti, 2005). The length of a path is represented by the number of nodes (network members) it contains (Borgatti et al., 2002). A short path directly links two members in a network (Kwon et al., 2007) whereas a long path also includes linkages to other members. Such direct linkage, as provided by a short path, allows for direct communication and therefore quick transfer of knowledge (particularly if the contact frequency is also high).

The shortest paths in Case 1 (see Figure 22) include, for example, the path connecting private businesses and universities or CRIs and central government. As this study's small-world networks represented advice networks, in which people most often knew whom they wanted to contact for seeking or giving knowledge, a considerable number of short paths were present. Figure 22 illustrates the structures of this study's three small-world networks. 


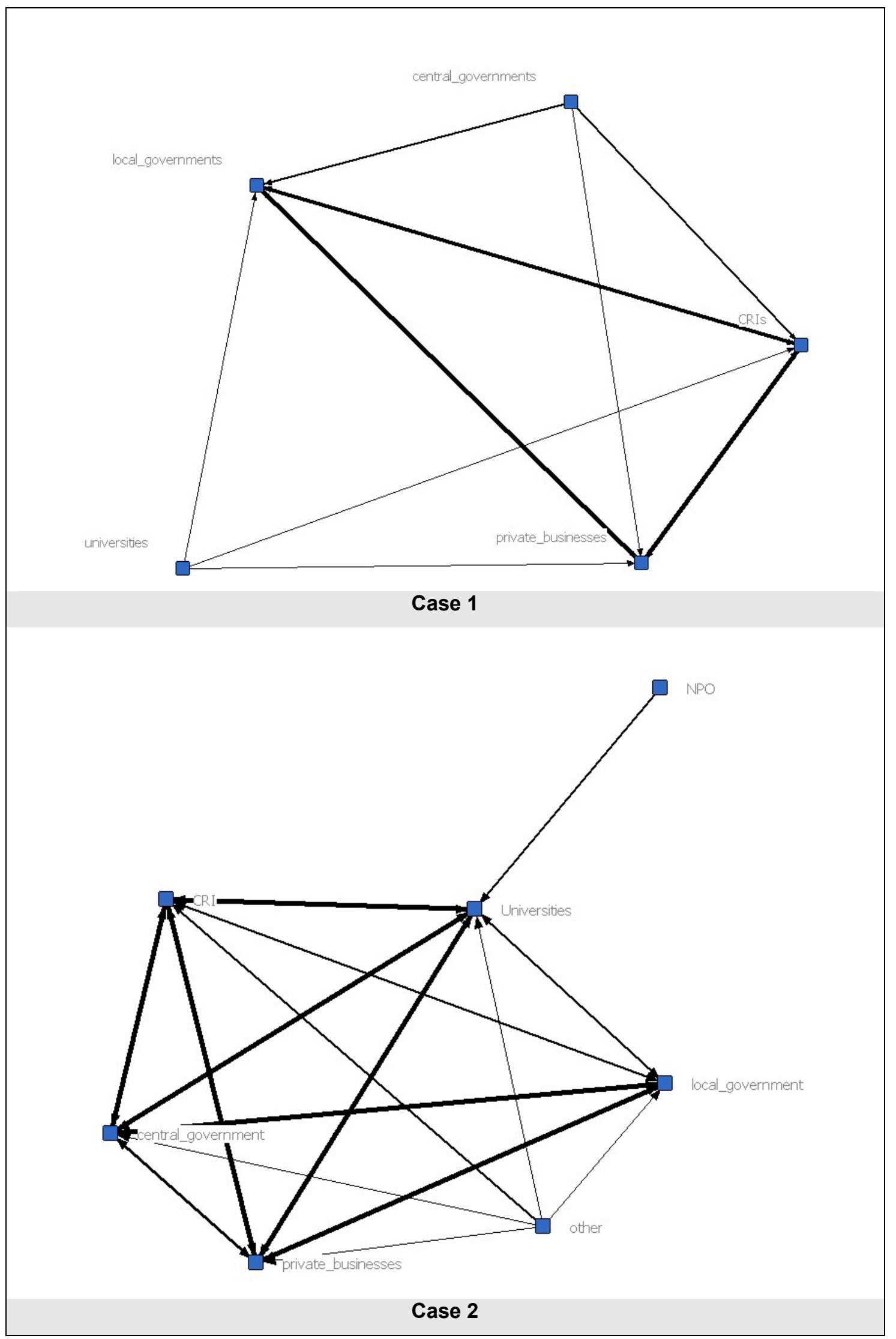




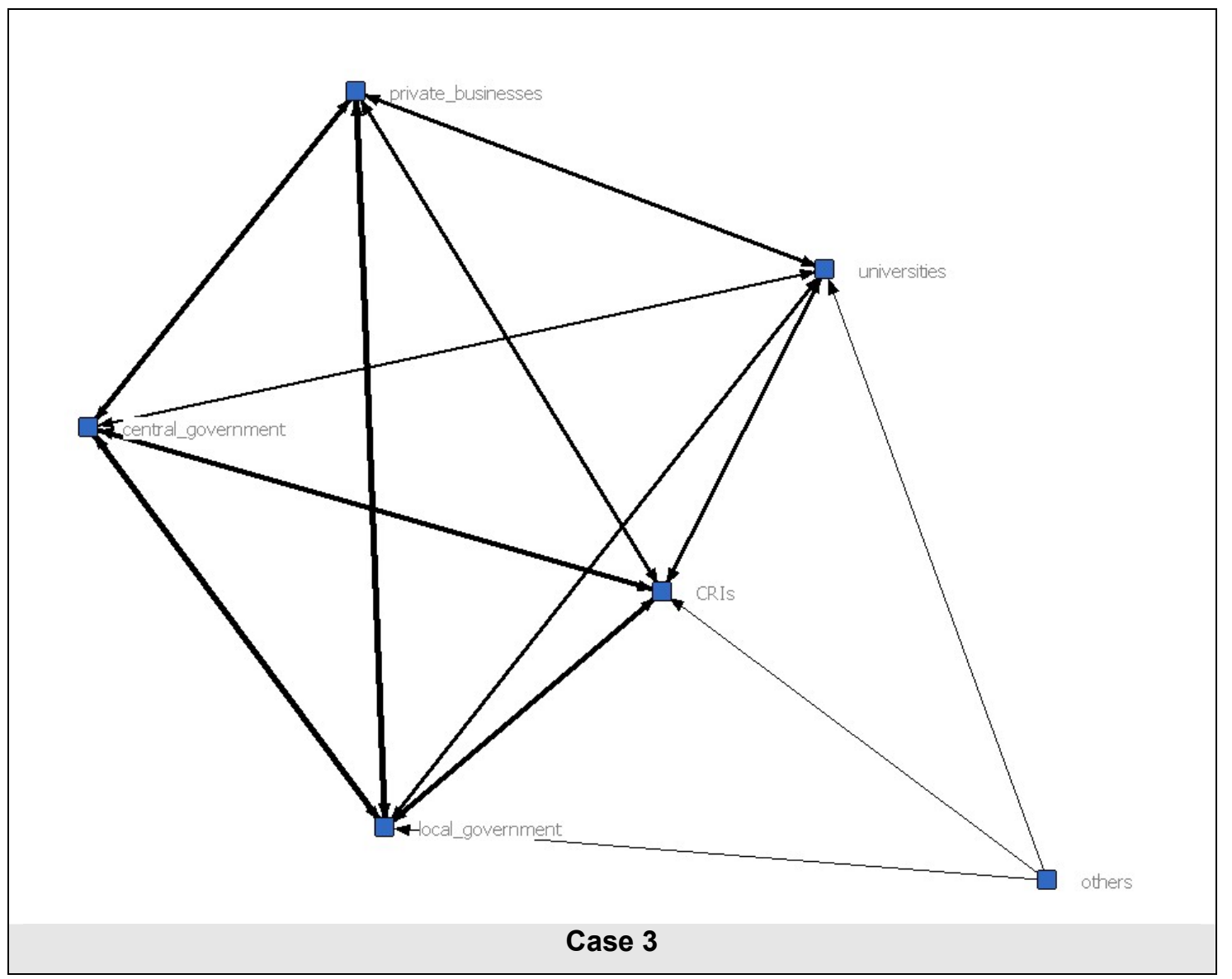

Figure 22: This study's three small-world networks in comparison

In Figure 22, the empirically observed tie strength is shown by the thickness of the lines.

\subsubsection{A high clustering coefficient}

Secondly, another typical characteristic for small-world networks is a high clustering coefficient which represents the high average of the connectivity among the neighbours of each network member (Kwon et al., 2007). Watts and Strogatz (1998) introduced the clustering coefficient measure in 1998. In particular, they determined that the neighbourhood connectivity in small-world networks is higher than in comparable random networks (Watts \& Strogatz, 1998). 
In Case 1, for example, the network's clustering coefficient was considerably higher (0.7) than the clustering coefficient in a random network of comparable size (0.27) as shown in Table 45. In addition, Table 45 illustrates the clustering coefficients for all three small-world network cases in relation to comparable random networks and the networks' overall densities. The measures were conducted by using Ucinet software. The binarised data sets underlying these measures can be found in Appendix 19.

Table 45: Clustering coefficients and network density

\begin{tabular}{|l|c|c|c|}
\hline & $\begin{array}{l}\text { Clustering } \\
\text { coefficient }\end{array}$ & $\begin{array}{l}\text { Clustering coefficient for a } \\
\text { comparable random network }\end{array}$ & $\begin{array}{l}\text { Overall network } \\
\text { density }\end{array}$ \\
\hline Case 1 & 0.70 & 0.27 & 0.60 \\
\hline Case 2 & 0.79 & 0.42 & 0.62 \\
\hline Case 3 & 0.85 & 0.54 & 0.77 \\
\hline
\end{tabular}

The numbers in Table 45 confirm that due to their dense local neighbourhoods, this study's small-world networks showed high clustering coefficients compared to random networks of the same size.

In a SNA diagram ${ }^{9}$, actors who belong to a local cluster can be identified by "an area of relatively high density in a graph" (Scott, 2000, p.127). The social network in Case 1, for example, showed such a local cluster including the groups of organisation which had the highest number of, and also strongest, connections to other types in the network. These groups of organisation, namely CRIs, local government and private businesses, were therefore in the best positions to facilitate tight collaboration and effective routine communications (Kwon et al., 2007).

In order to avoid an 'over-interpretation' of high clustering coefficients, Hanneman (2005) recommends a comparison of the clustering coefficient to the overall density of the network. The density is the total number of ties divided by the total number of

\footnotetext{
${ }^{9}$ as illustrated by Netdraw 2.08 in Figure 22, p.213/214
} 
possible ties (Borgatti et al., 2002) and therefore represents the average connectivity in the whole network.

The densities of the entire networks were also high as shown in Table 45. These findings reveal high network connectivities, which represent overall good opportunities for knowledge transfer, in addition to the high clustering coefficients. However, as the density results were high, the clustering coefficient values did not reflect an equally high value, as they would have in a network with low density. This means the opportunities for knowledge transfer within these local clusters were only slightly higher than in the whole network. This may be a result of the aggregation of organisations into groups of organisation.

The identification of all three social networks as small-world networks presents some important aspects for inter-organisational knowledge transfer. Due to the high clustering coefficients as well as the high number of short paths, study participants in the networks shared the advantage of having relatively easy access to interorganisational knowledge sources. Apart from inhibitors such as remote locations or competition issues, these small-world network topologies provided a reasonably well structured opportunity for inter-organisational knowledge transfer.

In addition to facilitating knowledge transfer, Schilling and Phelps (2007), for example, argue that a small-world network structure is the ideal structure to optimise the output (knowledge creation) of inter-firm knowledge networks such as alliances, as these structures allow for the right bandwidth (strength) and the appropriate reach (connectivity) among network members. Moreover, small-world networks were mentioned as the ideal network structure to facilitate knowledge transfer and creation in the knowledge diffusion literature (e.g. Cowan, 2004; Cowan \& Jonard, 2004). 


\subsubsection{Summary}

This section has identified three small-world networks. A small-world network represents a decentralised ontological network structure that includes some degree of local clustering. In this regard, this chapter has described, compared and illustrated the characteristics of the three social networks in this study including the high clustering coefficients and the high number of shortest paths. This network structure facilitates inter-organisational knowledge transfer.

\subsection{Intermediate ties}

This section defines and describes the important role of intermediate ties ${ }^{10}$ in the three small-world networks. Intermediate ties are relationships with a medium tie strength that neither fit into the group of strongest relationships nor in the group of weakest relationships in this study. A more detailed definition includes considerations about the classification of total tie strength values in a network and reciprocal knowledge transfer, which help to clearly separate the strength of intermediate ties from strong and weak ties. Firstly, an introduction outlines relevant literature and describes this study's tie strength scenario. Moreover, this section discusses the importance of intermediate ties in relation to their important role as boundary spanners in the inter-organisational networks. Supporting data for the definition of intermediate ties in each case are found in Appendix 20.

\subsubsection{Introduction}

Firstly, previous key literature which investigated tie strength, and particularly defined intermediate ties, is examined. Secondly, details of the tie strength measurement in this study are described.

\footnotetext{
${ }^{10}$ Besides intermediate, other possible terms used in previous literature are medium-strong or moderately strong ties (see Glossary for a definition of intermediate ties)
} 
In the past, SNA literature with a knowledge management context has often focussed on, or referred to, the importance of strong and/or weak ties rather than on intermediate ties (e.g. Granovetter, 1982; Hansen, 1999; Levin \& Cross, 2004). Although ties have often been referred to as being weak or strong, the exact definition of what exactly is weak or strong varies in particular contexts (Marsden \& Campbell, 1984). For example, strong ties can be defined by reciprocal contact, whereas weak ties are defined by asymmetrical contact (Friedkin, 1980; Granovetter, 1973).

Besides reciprocity, other indicators such as contact frequency (Borrego et al, 2007; Granovetter, 1974; Langlois, 1977), closeness (Dodds et al., 2003; Marsden \& Campbell, 1984), and amount of time (Onnela et al, 2007; Petróczi et al., 2007), have been employed to define tie strength. These SNA studies, mostly undertaken at the individual level, have often defined intermediate ties by the medium values of their scales used for data collection. For example, on a closeness scale ranging from 'not close' to 'extremely close', these medium values have been 'fairly close' or 'casual'.

In this study, however, SNA was conducted at the inter-organisational level, which resulted in a combination of contact frequency and number of study participants per group of organisation as the main indicators for tie strength. In terms of contact frequency, the direction of knowledge transfer (uni-directional values for advice giving and seeking activities) was considered. Table 46 outlines these uni-directional tie strength values between the different groups of organisation in Case 2 . Organisational relationships were aggregated at the group of organisational level. Data underlying these aggregations for each case are found in Appendix 21. 
Table 46: Case 2 uni-directional (reciprocal) tie strength values

\begin{tabular}{|c|c|c|c|c|c|c|c|}
\hline \multirow[b]{3}{*}{ from: } & \multicolumn{7}{|c|}{ Case 2 aggregated } \\
\hline & 1 & 2 & 3 & 4 & 5 & 6 & 7 \\
\hline & CRI & cen & Uni & pri & loc & NPO & oth \\
\hline $1 \mathrm{CRI}$ & 104 & 16 & 40 & 7 & 7 & 0 & 0 \\
\hline 2 central_government & 42 & 90 & 44 & 13 & 39 & 0 & 0 \\
\hline 3 Univer sities & 25 & 28 & 99 & 10 & 13 & 0 & 0 \\
\hline 4 private_businesses & 40 & 20 & 43 & 99 & 39 & 0 & 0 \\
\hline 5 local_government & 1 & 5 & 1 & 2 & 14 & 0 & 0 \\
\hline $6 \mathrm{NPO}$ & 0 & 0 & 8 & 0 & 0 & 0 & 0 \\
\hline 7 other & 7 & 2 & 6 & 2 & 1 & 0 & 0 \\
\hline
\end{tabular}

Table 46 illustrates the contact frequencies among the groups of organisation in each direction. For example, the contact frequency from CRIs to central government has a tie strength value of 16, whereas the transfer from central government to CRIs has a value of 42 . The overall strength of a tie is therefore defined by the sum of the unidirectional (reciprocal) values, which would be 58 in the example above. Whereas Table 46 shows only uni-directional tie strength values, Table 47 illustrates the bidirectional values as a summary of the uni-directional values and the connected groups of organisation. The bi-directional values represent the total tie strength, whereas the uni-directional values represent reciprocal values. 
Table 47: Values in relation to type of tie strength in Case 2

\begin{tabular}{|c|c|c|}
\hline $\begin{array}{l}\text { Uni-directional } \\
\text { (reciprocal) } \\
\text { values }\end{array}$ & $\begin{array}{c}\text { Bi-directional (total) } \\
\text { values }\end{array}$ & Type of tie \\
\hline $0+1$ & 1 & Local government - others \\
\hline $0+2$ & 2 & Central government - others \\
\hline $0+2$ & 2 & Private business - others \\
\hline $0+6$ & 6 & University - others \\
\hline $0+7$ & 7 & CRIs - others \\
\hline $0+8$ & 8 & Universities - NPOs \\
\hline $7+1$ & 8 & CRIs - local government \\
\hline $13+1$ & 14 & Universities - local government \\
\hline $20+13$ & 33 & Private businesses - central government \\
\hline $39+2$ & 41 & Private businesses - local government \\
\hline $5+39$ & 44 & Local governments - central government \\
\hline $7+40$ & 47 & CRIs - private businesses \\
\hline $10+43$ & 53 & Universities - private businesses \\
\hline $16+42$ & 58 & CRIs - central governments \\
\hline $40+25$ & 65 & CRIs - universities \\
\hline $28+44$ & 72 & Universities - central government \\
\hline
\end{tabular}

Table 47 shows the total (bi-directional) tie strength values in ascending order. For example, the bi-directional value for the relationship between CRIs and private businesses is 47 . In this case, the knowledge transfer from CRIs to private businesses was lower (7) than from private businesses to CRIs (40).

This section outlined the components of tie strength, bi-directional contact frequency and number of study participants per organisation in this study, and used Case 2 examples for illustration.

In the previous case write-ups (Chapters 4-6), the visualisation program Netdraw was employed to illustrate tie strength in this study's three networks. With investigating the line thickness of ties in the resulting Netdraw figures, it became apparent that, besides a number of strong and weak ties, there also existed a considerable number of ties of medium strength. However, no clear distinction criteria that defined these intermediate ties, or more importantly separated them from the strong and weak ties 
in the same network, could be recognised. Therefore, the following section aims to establish a numerical representation for the tie strength of intermediate ties.

Definition of the tie strength of intermediate ties

For defining the tie strength of intermediate ties, the following two criteria were considered important:

1. A distinct group of intermediate ties with maximum margins between the group of weak and intermediate ties as well as the group of intermediate and strong ties as well as minimum margins between ties within each group (k-means clustering method).

2. The level of reciprocity as an additional indicator to clearly distinguish intermediate from weak and strong ties at the borderlines of the initially identified group of intermediate ties.

These two criteria were applied in sequence. Employing the k-means clustering method for Criterion 1 provided an initial classification of all total tie strength values into three groups: weak, intermediate and strong ties. The group with the medium to high levels of tie strength was defined as the group of intermediate ties. This initial definition was followed and refined by applying Criterion 2. In particular, the borderlines of the initially defined intermediate ties group were therefore investigated.

\subsubsection{Criterion 1: k-means clustering for initial classification}

In regard to separating intermediate from weak and strong ties in a social network, previous studies were reviewed for clear distinction criteria. In particular, SNA 
studies from Granovetter (1973), Dodds et al. (2003) and Onnela et al. (2007) were considered as being important and are therefore described in the following sections. All three studies investigated social networks at the individual level and translated their scale items used for measuring tie strength into tie strength categories.

In Granovetter's (1974) case, frequency of contact and closeness were the most important indicators for tie strength. However, for classifying tie strength, contact frequency appeared to be the main indicator, as intermediate ties were defined as having an occasional contact frequency of "more than once a year but less than twice a week". Granovetter (1974) therefore defined intermediate ties by the scale used for data collection. In other words, he transferred the scale item(s) in the middle of the scale into the classification for intermediate ties. In his study, Granovetter (1974) investigated tie strength in relation to job-searching outcomes. Although he identified weak, intermediate and strong ties in his study, with a majority of intermediate ties, the notion of the strength of weak ties was considered more important in his study's context than the majority of intermediate ties.

Dodds et al. (2003), who investigated advice seeking activities, defined intermediate ties as friendships established by work or school affiliations that are casual or fairly close. In general, tie strength was measured by closeness, and origin of the relationship (e.g. work). Their results indicate that people in a small-world network seek help from intermediate ties rather than from weak ties. They state: "The most useful category of social tie is medium-strength friendships that originate in the workplace" (Dodds et al., 2003).

Further, Onnela et al. (2007), who investigated the structure and tie strength in mobile communication networks, defined intermediate ties by the amount of time people spent (100 seconds on a scale from 0 to 10,000$)$ to communicate. Thus tie strength was measured by the amount of time spent per contact. Most study participants indicated a timeframe of 100 seconds and were therefore categorised as intermediate ties. The scale items used for measuring tie strength were directly related to the 
classification of tie strength groups. Onnela et al. (2007) found that an individual learns about new knowledge through ties of intermediate strength rather than through weak or strong ties.

In summary, these three studies classified intermediate ties by the scale item(s) used for their SNA data collection. These scale items were mostly based on one single indicator for tie strength such as contact frequency, closeness or amount of time spent for communicating. This allowed for a relatively simple translation from scale item(s) into tie strength classifications. In addition, these three studies investigated social networks at the individual level. In conclusion, either the amount of intermediate ties was considerably large, or their purpose played an important role. These aspects indicate the significance of intermediate ties in social networks. Therefore, their correct definition appears crucial.

Although other studies often investigated tie strength by only one indicator, this thesis investigated social networks at an inter-organisational level and therefore had two indicators, which could not be initially combined in the scale items, for measuring tie strength. These indicators were contact frequency and the number of study participants per organisation. Therefore, a direct translation from scale item(s) into tie strength categories was not possible.

In this regard, one other study (Petróczi et al., 2007) could be identified that specifically describes tie strength measurement in social networks considering several indicators such as closeness, reciprocity and advice given/received. Petróczi et al. (2007) focussed on the development of a continuous tie strength scale. According to their study, Granovetter (1973) and many who referred to his research assumed ties to be dichotomous values (0/1), which resulted in the exclusive description of weak and strong ties. Therefore, Petróczi et al. (2007) specifically aimed to extend the previous distinction of weak and strong ties by considering more diverse levels of tie strength. They also considered asymmetric pairs of social relationships in their total tie strength distribution and classification among weak, medium and strong ties. 
In Petróczi et al.'s (2007) study, the total tie strength values were distinguished as weak, intermediate and strong ties. Ties were initially classified by using k-means clustering (A. Petróczi, personal communication, March 21, 2009). They finally defined medium ties as lying between the total tie strength values of 4 and 16 in a tie strength ranging from zero to 22. The cut-off point between intermediate and strong ties (17) was defined by the upper quartile. In addition, this cut-off point was explained by using closeness arguments. Medium ties represented acquaintanceships, whereas strong ties represented friendships.

However, the cut-off point between weak and intermediate ties was not defined by using such a numerical representation (quartile) as between intermediate and strong ties. Weak ties mostly included ties with no contact at all or few contacts. Most ties showed a weak strength (89.5\%), medium ties scored second (8.9\%), and strong ties came third (1.6\%). In line with this tie strength classification, Petróczi et al. (2007) showed a useful approach by grouping ties into weak, intermediate and strong ties.

In particular, the k-means clustering was also employed in this thesis to apply criterion one to group all tie strength values into three groups. The k-means clustering allows for an identification of homogeneous groups based on a specified number of clusters. Therefore, this clustering method assumes that the researcher knows the number of clusters. As the aim was to form three groups of tie strength - weak, intermediate and strong - three clusters were specified.

Another assumption made by the k-means clustering method is the use of a simple Euclidean distance for the classification. The Euclidean distance between two items, $\mathrm{x}$ and $\mathrm{y}$, is the square root of the sum of the squared differences between the values for the items. In this study's context, having simple one-dimensional data, this distance measure appeared suitable. One great advantage of the k-means clustering is that it can also handle large data-sets, which makes it transferable to other social network contexts. 
In summary, employing the k-means clustering method helped to group all total tie strength values into three groups, and therefore initially separate intermediate from weak and strong ties. For applying the k-means clustering method to this study's data-sets, the SPSS (16.0) standard software package for statistical analysis was used.

The k-means algorithm, as used in this study, follows three steps: an initial identification of cluster centres, the assignment of all cluster cases (total tie strength values) to the initial cluster centres, and a final recalculation of cluster centres. Applying these steps results in having cluster memberships for all cluster cases and maximum distances between cluster centres and minimum distances within clusters. For illustration purposes, the application of these steps will be described for Case 2 (page 226).

Firstly, Table 48 shows the cluster classification results for Case 1. The 'Cluster' column indicates the tie strength categorisation (cluster membership) for each tie strength value into weak, intermediate or strong. Supporting data can be found in Appendices 20 and 21.

Table 48: Classification of total tie strength values for Case 1

\begin{tabular}{|c|c|c|}
\hline $\begin{array}{c}\text { Total (reciprocal) } \\
\text { tie strength } \\
\text { values }\end{array}$ & Type of tie & Cluster \\
\hline $1(0+1)$ & Universities - local government & weak \\
\hline $2(2+0)$ & Universities - private businesses & weak \\
\hline $4(0+4)$ & CRIs - universities & weak \\
\hline $7(7+0)$ & Private businesses - central government & weak \\
\hline $13(13+0)$ & Local government - central government & weak \\
\hline $19(0+19)$ & CRIs - central government & weak \\
\hline $68(55+13)$ & Private businesses - local government & intermediate \\
\hline $69(17+52)$ & CRIs - private businesses & intermediate \\
\hline $90(48+42)$ & CRIs - local government & strong \\
\hline
\end{tabular}


For Case 1 the computed final cluster centres were 7.5 for the group of weak ties, 68.5 for the group of intermediate ties, and 90 for the group of strong ties. Of all ties, 22 percent could initially be identified as intermediate ties in this network. Intermediate tie strength values in relation to the highest tie strength value in the network ranged from 75.5 to 76.7 percent.

In order to achieve the resulting cluster memberships of all cluster cases, the k-means algorithm, as applied in this study, follows three steps:

- an initial identification of cluster centres;

- the assignment of all cluster cases (total tie strength values) to these initial cluster centres; and

- a final recalculation of cluster centres.

For Case 2 (results in Table 52), SPSS calculated the following initial cluster centres:

Table 49: Initial cluster centres in Case 2

\begin{tabular}{|l|c|c|c|}
\hline \multicolumn{4}{|c|}{ Initial Cluster Centres } \\
\hline & \multicolumn{3}{|c|}{ Cluster } \\
\hline & 1 & 2 & 3 \\
\hline VAR00001 & 1.00 & 33.00 & 72.00 \\
\hline
\end{tabular}

The algorithm's first pass through the data resulted in a definition of the initial cluster centres which indicates a maximum distance among the three centres (based on cluster means). Next, each cluster case (total tie strength value) is assigned to one of the centres to which it has a minimum distance (nearest cluster). Then, cluster means are updated. Finally, the third pass through the data assigns each case to the nearest cluster in regard to the Euclidean distance between that case and the updated cluster means. Final cluster means are then calculated. These calculations provide the final cluster centres, which, in Case 2, are: 
Table 50: Final cluster centres in Case 2

\begin{tabular}{|c|c|c|c|}
\hline \multicolumn{4}{|c|}{ Final Cluster Centres } \\
\hline & \multicolumn{3}{|c|}{ Cluster } \\
\hline & 1 & 2 & 3 \\
\hline VAR00001 & 6.00 & 41.25 & 62.00 \\
\hline
\end{tabular}

This final calculation also provides final cluster memberships for all cluster cases. These are shown in Table 51. 'Case Number' shows the number of total tie strength values for Case 2, 'Cluster' refers to the actual membership ( $1=$ weak, $2=$ intermediate, $3=$ strong) and 'Distance' indicates the distance to the respective cluster centre.

Table 51: Cluster memberships in Case 2

\begin{tabular}{|c|c|c|}
\hline \multicolumn{3}{|c|}{ Cluster Membership } \\
\hline Case & & \\
\hline Number & Cluster & Distance \\
\hline 1 & 1 & 5.000 \\
\hline 2 & 1 & 4.000 \\
\hline 3 & 1 & 4.000 \\
\hline 4 & 1 & .000 \\
\hline 5 & 1 & 1.000 \\
\hline 6 & 1 & 2.000 \\
\hline 7 & 1 & 2.000 \\
\hline 8 & 1 & 8.000 \\
\hline 9 & 2 & 8.250 \\
\hline 10 & 2 & .250 \\
\hline 11 & 2 & 2.750 \\
\hline 12 & 2 & 5.750 \\
\hline 13 & 3 & 9.000 \\
\hline 14 & 3 & 4.000 \\
\hline 15 & 3 & 3.000 \\
\hline 16 & 3 & 10.000 \\
\hline
\end{tabular}


In Table 51, the first eight cluster cases belong to the cluster of weak ties (1). The next four cluster cases belong to the cluster of intermediate ties (2), and the final four cluster cases belong to the cluster of strong ties (3).

The application of this k-means clustering algorithm allowed for the classification of all total tie strength values in Case 2 which is illustrated below.

Table 52: Classification of total tie strength values for Case 2

\begin{tabular}{|c|c|c|}
\hline $\begin{array}{c}\text { Total (reciprocal) } \\
\text { tie strength values }\end{array}$ & Type of tie & Cluster \\
\hline $1(0+1)$ & Local government - others & weak \\
\hline $2(0+2)$ & Central government - others & weak \\
\hline $2(0+2)$ & Private business - others & weak \\
\hline $6(0+6)$ & University - others & weak \\
\hline $7(0+7)$ & CRIs - others & weak \\
\hline $8(0+8)$ & Universities - NPOs & weak \\
\hline $8(7+1)$ & CRIs - local government & weak \\
\hline $14(13+1)$ & Universities - local government & weak \\
\hline $33(20+13)$ & Private businesses - central government & intermediate \\
\hline $41(39+2)$ & Private businesses - local government & intermediate \\
\hline $\mathbf{4 4}(\mathbf{5 + 3 9 )}$ & Local government - central government & intermediate \\
\hline $\mathbf{4 7}(\mathbf{7 + 4 0 )}$ & CRIs - private businesses & intermediate \\
\hline $53(10+43)$ & Universities - private businesses & strong \\
\hline $58(16+42)$ & CRls - central government & strong \\
\hline $65(40+25)$ & CRIs - universities & strong \\
\hline $72(28+44)$ & Universities - central government & strong \\
\hline
\end{tabular}

For Case 2 the computed cluster centres were 6 for the group of weak ties, 41.25 for the group of intermediate ties, and 62 for the group of strong ties. Of all ties, 25 percent could initially be identified as intermediate ties in this network. Intermediate tie strength values in relation to the highest tie strength value in the network ranged from 45.8 percent to 65.3 percent.

Finally, Table 53 illustrates the results for Case 3. 
Table 53: Classification of total tie strength values for Case 3

\begin{tabular}{|c|c|c|}
\hline $\begin{array}{c}\text { Total } \\
\text { (reciprocal) tie } \\
\text { strength values }\end{array}$ & Type of tie & Cluster \\
\hline $1(0+1)$ & CRIs - others & \\
\hline $1(0+1)$ & Universities - others & weak \\
\hline $2(0+2)$ & Local government - others & weak \\
\hline $19(13+6)$ & Universities - central government & intermediate \\
\hline $24(8+16)$ & Universities - private businesses & intermediate \\
\hline $28(22+6)$ & CRIs - universities & intermediate \\
\hline $32(23+9)$ & CRIs - central government & intermediate \\
\hline $34(14+20)$ & Universities - local government & intermediate \\
\hline $36(19+17)$ & CRIs - private businesses & intermediate \\
\hline $43(29+14)$ & Private businesses - central government & strong \\
\hline $56(30+26)$ & CRIs - local government & strong \\
\hline $60(25+35)$ & Private businesses - local government & strong \\
\hline $61(36+25)$ & Local government - central government & strong \\
\hline
\end{tabular}

For Case 3 the computed cluster centres were 1.33 for the group of weak ties, 28.83 for the group of intermediate ties, and 55 for the group of strong ties. Of all ties, 38 percent could initially be identified as intermediate ties in this network. Intermediate tie strength values in relation to the highest tie strength value in the network ranged from 31.1 percent to 59 percent.

In summary, the overall percentage range of initial intermediate ties in relation to the highest tie strength in a network could be revealed by considering all three cases. This percentage range then goes from a minimum of 31.3 percent to a maximum of 76.7 percent, which would indicate a 45.4 percent range for intermediate ties within a network. However, the mean percentage of the number of intermediate ties in a network was lower and could be identified in this context as 28.33 percent.

After initially defining the group of intermediate ties through employing the k-means clustering, the application of the second criterion - reciprocity - aimed to refine the borderlines of this group. This is explained and illustrated with Case 2 examples in 
the following section. Supporting data for Cases 1 and 3 can be found in Appendix 20.

\subsubsection{Criterion 2: Reciprocity}

Besides initially identifying the group of intermediate ties, this study applied reciprocity as an additional indicator for intermediate tie strength. A number of previous studies have highlighted the importance of reciprocity for tie strength and knowledge transfer (e.g. Bresman et al., 1999; Friedkin, 1980; Granovetter, 1973; Hansen, 1999; Petróczi et al., 2007).

For example, Friedkin (1980), who investigated the discussions in a research network among faculty members in biological science departments, highlighted the importance of reciprocal ties for tie strength, besides other factors such as amount of time, the emotional intensity, and intimacy (mutual confiding). Using these indicators for tie strength, he agreed with Granovetter (1973) who recognised the significance of reciprocity as one distinctive feature of strong ties.

The importance of reciprocity can be recognised by adjusting the weighting of the tie strength score in which such a mutual recognition occurred (Petróczi et al., 2007). In this case, a tie can be double-weighted to indicate a stronger tie. Therefore, strong ties show a high reciprocity, whereas weaker ties tend to be less reciprocal (Petróczi et al., 2007).

In the context of this study, reciprocal ties would also mean reciprocal (bi-directional) knowledge transfer that may facilitate knowledge transfer more than non-reciprocal transfer. Reciprocal knowledge transfer (knowledge exchange) allows for discussion and may result in explanations and confirmations and thus facilitate the actual application of knowledge after the transfer (e.g. Bresman et al., 1999). 
Further, Hansen (1999) investigated the importance of strong, and therefore reciprocal, ties in the context of knowledge transfer. Strong ties can transfer complex knowledge better than weak ties (Hansen, 1999). This finding indicates the significance of reciprocity for knowledge transfer in knowledge-intensive industries such as the R\&D sector where it is likely that complex knowledge is transferred.

In this thesis, the level of reciprocity was considered for investigating ties at the borderlines of the initially defined group of intermediate ties. In the light of previous investigations, this study applied the level of reciprocity criterion as follows: between weak and intermediate ties, low reciprocity indicated a weak tie, whereas high reciprocity indicated an intermediate tie; between intermediate and strong ties, low reciprocity indicated an intermediate tie of lower strength. What low reciprocity actually represents needs to be defined by the researcher for the specific network context. In this study, a low level of reciprocity at the borderlines of intermediate ties is defined by two uni-directional values, in which one value is less than half of the second value. In Case 2, for example, a low level of reciprocity can consist of the values 43 and 10 in Table 54 (bi-directional value is 53 for this universities-private businesses connection), in which one value (10), is less than half of the second value (43).

Such a low reciprocity in this study may have been due to two circumstances: lower contact frequency for giving and/or seeking advice activities, or fewer study participants from one group of organisation. For example, study participants working at CRIs might have given monthly advice to people at private businesses, but study participants from private businesses might have given advice on a 6-monthly basis to people working at CRIs. This second condition, unbalanced reciprocity, may have also been due to fewer study participants from one of the two groups of organisation linked to each other. For instance, study participants working at CRIs or private businesses might have given advice to each other with the same strong contact frequency (e.g. monthly), but far more people participated from CRIs than private businesses. Then, the reciprocity from CRIs to private businesses would have been 
higher than from private businesses to CRIs. This indicates an unbalanced (unidirectional) knowledge transfer.

Applying criterion two to Case 2, the ties with a tie strength of $53(10+43)$ and 58 $(16+42)$ were also defined as intermediate ties due to their low level of reciprocity. In both connections, the lower value is less than half of the higher value. Thus, the level of reciprocity is defined as being low. All new intermediate ties are highlighted in red in Table 54.

Table 54: Final classification of total tie strength values for Case 2

\begin{tabular}{|c|c|c|}
\hline $\begin{array}{l}\text { Total (reciprocal) } \\
\text { tie strength values }\end{array}$ & Type of tie & Cluster \\
\hline $1(0+1)$ & Local government - others & weak \\
\hline $2(0+2)$ & Central government - others & weak \\
\hline $2(0+2)$ & Private business - others & weak \\
\hline $6(0+6)$ & University - others & weak \\
\hline $7(0+7)$ & CRIs - others & weak \\
\hline $8(0+8)$ & Universities - NPOs & weak \\
\hline $8(7+1)$ & CRIs - local government & weak \\
\hline $14(13+1)$ & Universities - local government & weak \\
\hline $33(20+13)$ & Private businesses - central government & intermediate \\
\hline $41(39+2)$ & Private businesses - local government & intermediate \\
\hline $44(5+39)$ & Local government - central government & intermediate \\
\hline $47(7+40)$ & CRIs - private businesses & intermediate \\
\hline $53(10+43)$ & Universities - private businesses & intermediate \\
\hline $58(16+42)$ & CRIs - central government & intermediate \\
\hline $65(40+25)$ & CRIs - universities & strong \\
\hline $72(28+44)$ & Universities - central government & strong \\
\hline
\end{tabular}

Table 54 indicates that, together with eight weak and two strong ties, six intermediate ties could finally be identified. In addition, Appendix 22 illustrates this tie strength distribution for Case 2. Examples of intermediate ties in Case 2 were the links between private businesses and CRIs or private businesses and central government. Figure 23 illustrates these examples together with the different levels of tie strength in the small-world network of Case 2. 


\section{Level of tie strength}

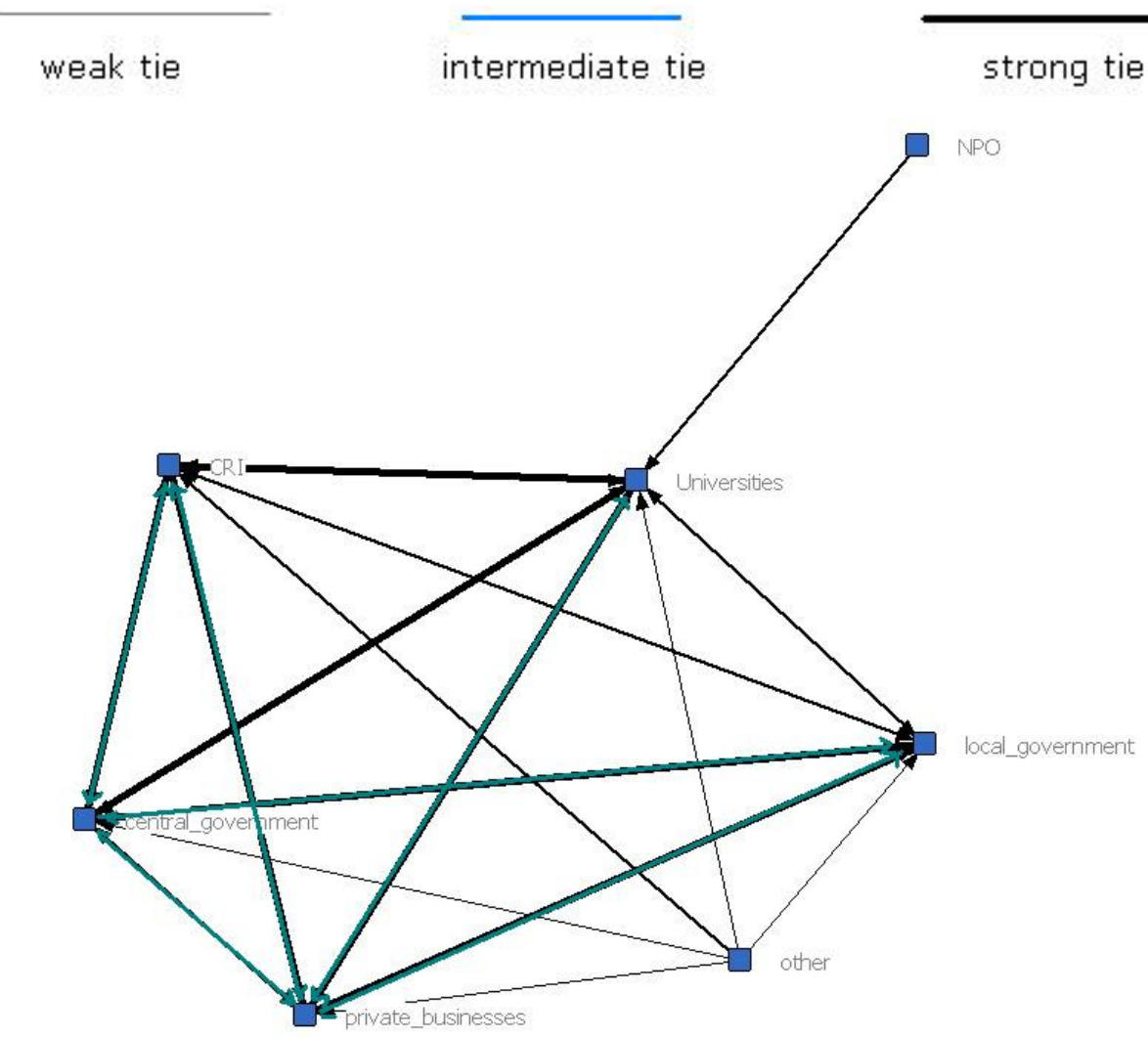

Figure 23: Case 2 small-world network

The result of applying these two criteria - the initial k-means clustering and reciprocity - to this study's data-sets is a percentage amount of intermediate ties of 37.5 in Case 2, 22 in Case 1 and 53.8 in Case 3. In this regard, the mean value is 37.7 percent for intermediate ties in a network, considering all three cases.

At an individual level, intermediate ties in Case 2 could, for example, represent friendships or acquaintanceships that were formed through previous work activities or schooling relationships, as suggested by Dodds et al. (2003). The existence of these rather informal relationships was confirmed by a Case 2 participant: "Great range of different types of interactions - some formal, some informal" (Participant 1). A Case 3 participant also made a comment in this regard: "We are linked by formal (e.g. contract work, research partnership) and informal (friendships, common interests) means" (Participant 2). 
When comparing the intermediate ties' efficiency to serve as sources for new knowledge, this relevant new knowledge was more likely to be transferred via intermediate than weak ties in this study's advice networks. However, Granovetter (1973) highlights the strength of weak ties and explains their influence on the transfer of new knowledge for job searching. For example, weak ties may be the better sources in this case, but overall the strength of weak ties appears rather contextspecific. Intermediate ties might often be based on longer (friendships) and more professionally related relationships (e.g. origin of relationship at work) rather than on loose social connections as with weak ties. Therefore, knowledge transferred by intermediate ties might be more relevant. In addition, Dodds et al. (2003) indicate that people in a small-world network seek help particularly from intermediate ties.

At the inter-organisational level, however, intermediate ties may represent a customer/supplier relationship. Due to specific interests, either the supplier or the customer contacts the other one more often, which would represent a low level of reciprocity. In order to facilitate these relationships at the inter-organisational level, they could for example be supported by corporate memberships in professional societies or other professional social gatherings (e.g. roundtables, conferences) in which individuals represent their group of organisation.

Summing up, the strength of intermediate ties could be characterised by two criteria. Firstly, all total tie strength values within one network were classified by using the kmeans clustering. This helped to initially identify the group of intermediate ties. Secondly, the borderlines of this group were investigated for the levels of reciprocity. Uneven (low) uni-directional (reciprocal) values at the borderlines always indicated a weaker tie (weak or intermediate tie). Applying these criteria, intermediate ties could be clearly distinguished from weak and strong ties. 
A considerable number of intermediate ties were revealed in each of the three smallworld networks. The remainder of this section describes the important roles of these intermediate ties in the networks.

\subsubsection{The important roles of intermediate ties}

Two particular roles of intermediate ties were identified in the three small-world networks.

Role 1: linking otherwise weakly connected groups of organisation to the network.

Examples for Role 1 existed in all three small-world social networks. In Case 1, private businesses were connected by intermediate ties to local government and CRIs, which were identified as key players ${ }^{11}$. These two intermediate ties are illustrated in blue in Figure 24. Otherwise, private businesses were only connected by rather weak relationships.

In Case 2, central government agencies were connected with local government, private businesses and CRIs by intermediate ties. Moreover, private businesses were exclusively linked by intermediate ties. These ties are also illustrated in blue in Figure 24.

In Case 3, universities were exclusively linked by intermediate ties, for example, to private businesses, central and local government (also shown in blue in the figure below). Overall, most of these intermediate ties helped prevent several groups of organisation from being connected weakly to the (other) key players in the networks (CRIs, local government and private businesses). These examples are illustrated in Figure 24.

${ }^{11}$ Section 7.3.1 


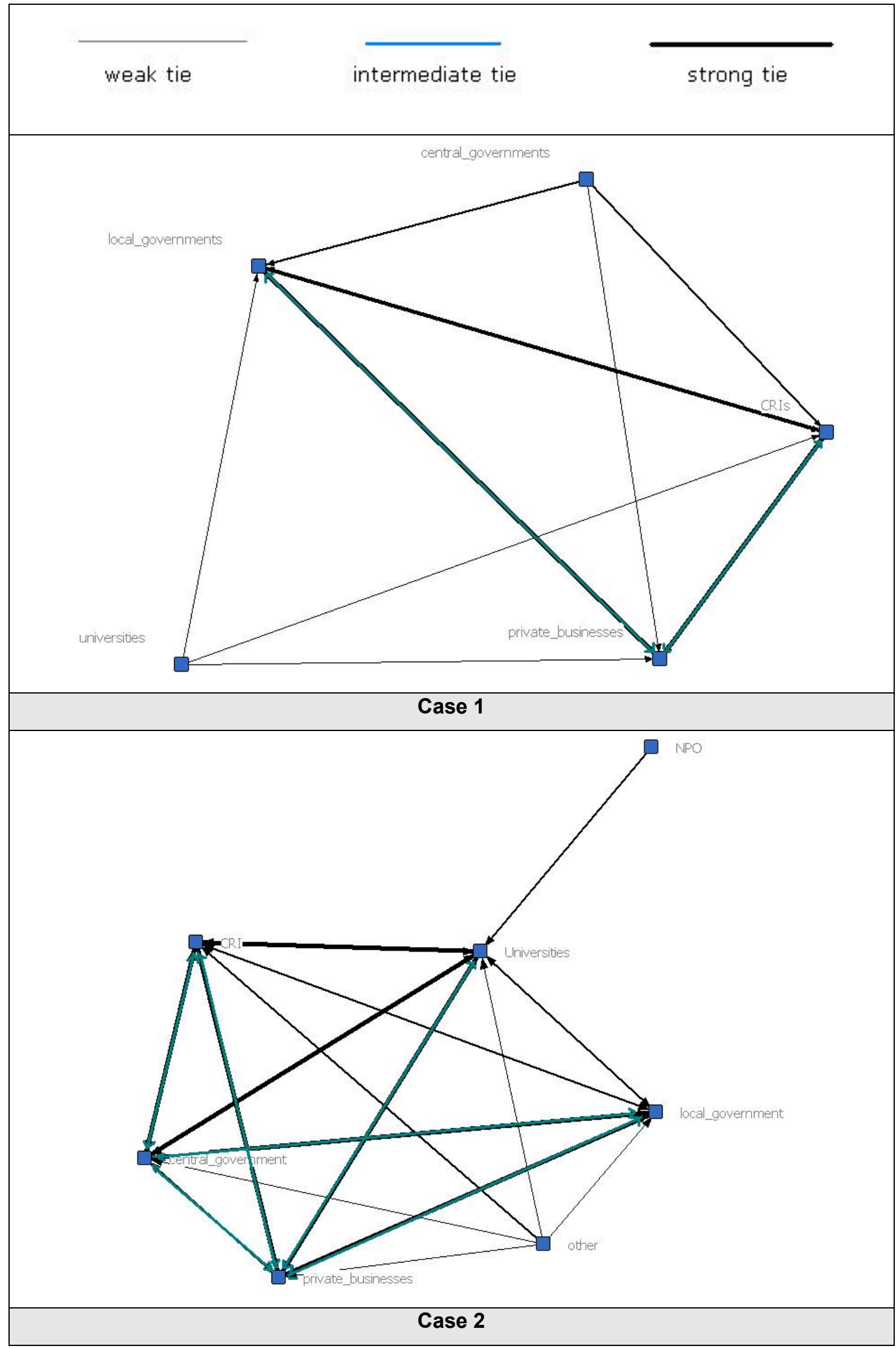




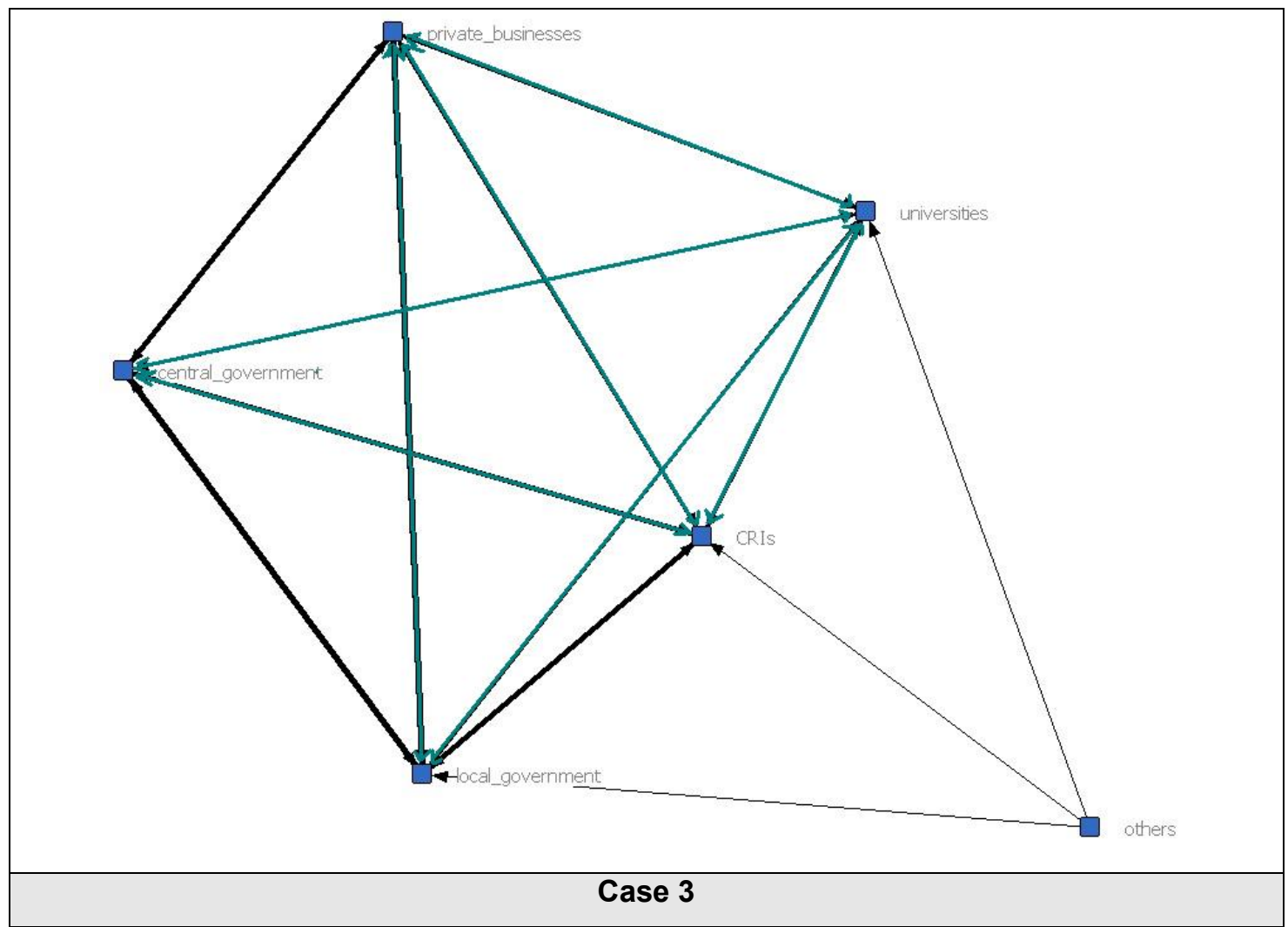

Figure 24: This study's three small-world networks in comparison

Role 2: linking two key groups of organisation which are well connected to other groups of organisation.

Examples of Role 2, which describes the linkage between two key organisations which are otherwise well-connected, could also be revealed in the three small-world social networks. In Case 1, two intermediate ties connected the three key players. These connections were between private businesses and CRIs as well as local government. In Case 2, an intermediate tie connected two of the three key players, private businesses with local government and with CRIs. In Case 3, an intermediate tie connected private businesses and CRIs as well as private businesses and local government. All these key players were well connected to other groups of organisation. 


\section{The inter-organisational context}

In relation to inter-organisational knowledge transfer, both roles appeared important. A Role 1 tie, which linked otherwise weakly connected groups of organisation, prevented some groups of organisation from being excluded from knowledge transfer in the network. Having a high number of intermediate ties in the context of this study, the category of private businesses represented such a group of organisation in Cases 1 and 2 .

In Case 3, universities were completely dependent on their intermediate connections to other groups of organisation. Moreover, through connecting otherwise weakly connected groups of organisation, Role 1 ties could help integrate new knowledge from external sources outside the network. For example, private businesses in the field or universities might have been active in other projects with organisations outside the network. This knowledge may then be of relevance to other network members, as it could help them develop new ideas.

However, Role 2 ties appear even more important, as such intermediate ties connected the key players in the small-world network, for example CRIs, central government and private businesses in Case 2. In this regard, intermediate ties appeared even more important than strong ties. A Role 2 tie not only gives key player 1 the opportunity to exchange knowledge with key player 2, but this kind of tie may also allow key player 1 to connect to the other organisations to which key player 2 is strongly connected.

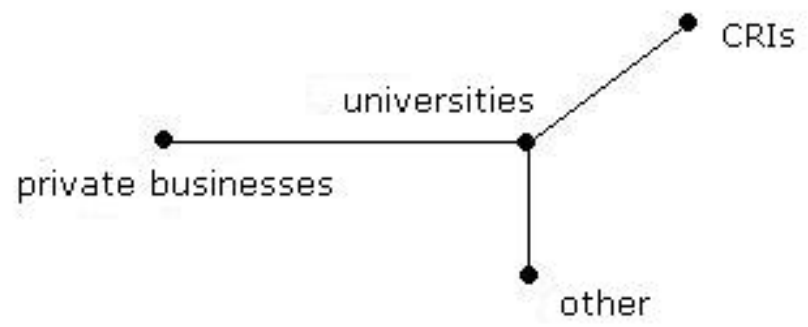

Figure 25: : Role 2 example 
In Case 2, for example, private businesses were connected to universities by an intermediate tie. Universities, moreover, were strongly connected to CRIs. This connection may help private businesses to achieve better contacts to CRIs. Intermediate ties therefore helped groups of organisation into a strategically important network position, in which the linked groups of organisation receive new or reusable knowledge early (Onnela et al., 2007).

\section{Boundary spanning characteristics}

Both roles of the intermediate ties indicated boundary-spanning characteristics. The boundary-spanning role is an informal position that typically belongs to an individual or an actor in a network rather than a tie (e.g. Hexmoor et al., 2006; Maier, 2001). Boundary spanners are concerned with maintaining or initiating social ties with other individuals or organisations (Adams, 1976; Aldrich \& Herker, 1977; Calloway, Morrissey \& Paulson, 1993; Mitchell, 2006). This includes formal and informal communications with external sources (Mitchell, 2006).

However, a network tie represents a 'local bridge' between two actors in a network (Granovetter, 1973). Therefore network ties, and particularly key ties such as this study's intermediate ties, have the bridging character that boundary spanners typically have. The direct linkage between two groups of organisation, particularly key types, may allow for a shorter and easier knowledge transfer and for engaging in new social relationships to foster knowledge transfer and learning.

This boundary-spanning characteristic of intermediate ties may also facilitate knowledge transfer. For example, in Case 2, CRIs and private businesses were connected by an intermediate tie. Therefore, CRIs may also have access to the local network around the private businesses that included central government agencies and universities. In return, private businesses may have access to knowledge transferred in the close network around CRIs that included local government and universities. This opportunity for accessing the neighbours' networks and thus using shorter paths 
or better maintained ties to transfer knowledge may be crucial. For example, private businesses could access the CRIs' strong ties to universities and therefore source cutting-edge research knowledge from universities. With the help of intermediate ties, knowledge transfer may also be quicker, which could be important when considering competition processes in the network.

\subsubsection{Summary}

In summary, this section defined the criteria for the strength of intermediate ties and described their importance in the small-world networks of this study. Intermediate ties were initially defined by employing k-means clustering to all tie strength values. Then, the borderlines of the group of initially identified intermediate ties were investigated for their levels of reciprocity. Each of the three small-world networks indicated a number of these intermediate ties. They therefore constituted the structures of inter-organisational knowledge transfer in the small-world networks.

Moreover, the intermediate ties had key roles in this study's social networks. Role 1 ties connected otherwise weakly linked groups of organisation. Role 2 ties linked two key players in the network. In addition, intermediate ties showed boundary-spanning characteristics in linking the networks' key players as well as linking otherwise weakly connected groups of organisation. Overall, a well-connected and balanced inter-organisational knowledge transfer could be identified. Besides this description of the intermediate ties, the comparison of all three networks also revealed several key roles, namely key sinks, key sources, knowledge brokers and the most influential groups of organisation, as part of the inter-organisational knowledge transfer. These are explained and illustrated in the following section. 


\subsection{Key players in the small-world networks}

This section introduces key players represented by groups of organisation with key aspects regarding their structural properties in the small-world networks of this study. Four key roles were identified:

- key sinks

- key sources

- key knowledge brokers

- influential roles according to Information Centrality values

Firstly, the first three roles - key sinks, key sources and key knowledge brokers - are defined and described in relation to the levels of interaction in the networks case by case. These descriptions relate strongly to the key findings in regard to the levels of interaction as presented in Chapters 4-6 (Sections 4.3.4, 5.3.1 and 6.3.1). Secondly, the fourth key role, referring to the more influential groups of organisation in regard to high Information Centrality values, is illustrated.

The analysis of the levels of interaction ${ }^{12}$ among network members revealed key initiators of relationships (key sources), key receivers of relationships (key sinks) and key knowledge brokers. As defined in the individual case write-ups ${ }^{13}$, key sources have represented knowledge seekers in this study's context (they initiate relationships), whereas key sinks have represented knowledge givers (they receive relationships). Therefore, key sinks represent leaders in their knowledge domain. Moreover, some of this study's groups of organisation represented both roles, key sinks and key sources, at the same time and were therefore both active givers and seekers of knowledge. These types represented the key knowledge brokers in the small-world networks.

\footnotetext{
12 Univariate Statistics analysis with Ucinet 6.188

13 See Chapters $4-6$
} 
The knowledge broker helps people to find specific knowledge or to identify experts (Ruggles, 1998). The knowledge broker typically sits at the intersection between, for example, two organisations or communities (Pawlowski \& Robey, 2004). Knowledge brokers are described as being part of several communities at the same time which allows them to broker the knowledge they receive from diverse sources. They are strategically positioned to facilitate knowledge transfer (Pawlowski \& Robey, 2004).

In this study, the key knowledge broker sat at the intersection of other key sinks and key sources. Key knowledge brokers were therefore key sinks and key sources at the same time. As the key knowledge brokers were well integrated in the networks, they were also well positioned to facilitate knowledge transfer, for example, in the form of providing learning opportunities to other network members. Key knowledge brokers were identified in two of the three networks. CRIs were the key knowledge brokers in Case 1, whereas local government and private businesses were the two key brokers in Case 3. In regard to their high knowledge giving and seeking activities, the key brokers provided the most sustainable and up-to-date knowledge bases within their networks.

Firstly, these key roles - key sinks, key sources and key knowledge brokers - are outlined and illustrated in the following three case-by-case descriptions. Then, a fourth key role, the most influential roles according to Information Centrality values, is described in summary for all three cases.

\subsubsection{Case 1 key players}

Key sources, key sinks and key knowledge brokers were all identified by their high mean and sum values for levels of interaction as illustrated in the following tables that show supporting data from the underlying univariate statistical analysis. Table 55 shows the mean values and the standard deviations (Std. Dev.) for these levels of interaction for Case 1. CRIs and local government emerged as the main sinks or 
knowledge givers. They each have a high mean value of 29.25 in their levels of interaction (by column), which represents key players that receive a high number of relationships (key sinks). These high values indicate authoritative positions in the network (Brogan \& Armstrong, 2005). In regard to knowledge transfer, key sinks represented the key knowledge givers in the network.

Table 55: Small-world network 1: Level of interaction by column (key sinks)

\begin{tabular}{|l|l|l|l|l|l|}
\hline & CRIs & $\begin{array}{l}\text { Private } \\
\text { businesses }\end{array}$ & $\begin{array}{l}\text { Local } \\
\text { government }\end{array}$ & $\begin{array}{l}\text { Central } \\
\text { government }\end{array}$ & Universities \\
\hline Mean & $\mathbf{2 9 . 2 5}$ & $\mathbf{9 . 7 5}$ & $\mathbf{2 9 . 2 5}$ & $\mathbf{0 . 0 0}$ & $\mathbf{0 . 0 0}$ \\
\hline Std. Dev. & 18.86 & 5.72 & 22.79 & 0.00 & 0.00 \\
\hline
\end{tabular}

The standard deviation is a measure of how much variation in interaction there is among the groups of organisation in the network. In particular, high standard deviation values, as shown in Table 55 for CRIs (18.86) and local government (22.79), indicate a high variability in how these groups of organisation are perceived by other types in regard to their knowledge giving activities. Different levels of tie strength among these groups of organisation in the network were the reason for these high variabilities.

To complement the level of interaction data shown in Table 55 (knowledge giving activities), Table 56 illustrates the statistical details which reflect the knowledge seeking activities in Case 1.

Table 56: Small-world network 1: Level of interaction by row (key sources)

\begin{tabular}{|l|l|l|l|c|c|}
\hline & CRIs & $\begin{array}{l}\text { Private } \\
\text { businesses }\end{array}$ & $\begin{array}{l}\text { Local } \\
\text { government }\end{array}$ & $\begin{array}{l}\text { Central } \\
\text { government }\end{array}$ & Universities \\
\hline Mean & $\mathbf{1 6 . 2 5}$ & $\mathbf{2 6 . 7 5}$ & $\mathbf{1 3 . 7 5}$ & $\mathbf{9 . 7 5}$ & $\mathbf{1 . 7 5}$ \\
\hline Std. Dev. & 19.60 & 26.77 & 17.15 & 7.05 & 1.48 \\
\hline
\end{tabular}

In Table 56, the highest activity in initiating relationships (seeking knowledge) is shown by private businesses $($ mean $=26.75)$ which were therefore key sources in the network. CRIs and local government agencies also show relatively high values. Central government and universities indicate a low level of interaction in terms of 
knowledge seeking. More results from this univariate statistical analysis for network 1 are found in Appendix 23.

The following matrix illustrates the distribution of the key players in Case 1 regarding their levels of knowledge transfer. The key sinks are illustrated in the top left corner, whereas the key sources are placed within the lower right corner. The key knowledge brokers are illustrated in the top right corner. The knowledge brokers who indicated a lower level of interaction than the key knowledge brokers are shown in the lower left corner.

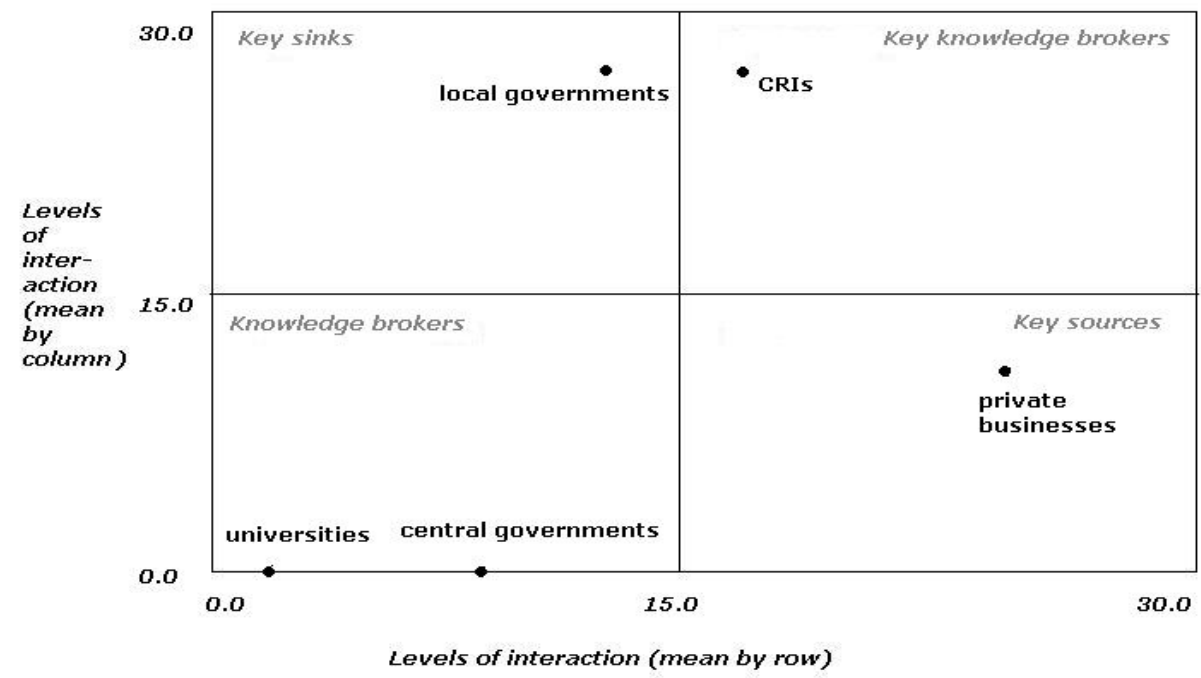

Figure 26: Small-world network 1: Matrix of key sinks and key sources

Figure 26 reveals the private businesses as the key knowledge seekers (key sources). This may indicate that these organisations need domain knowledge, for example from CRIs and local government, as an input into their daily work to develop supporting instruments for environmental data collection and analysis. Another reason for private businesses to collect knowledge from diverse sources could be their motivation to provide summaries and more substantial forecasts to clients. However, it could also simply be the fact that private businesses did not give advice informally, but rather aimed to sell their knowledge in the form of consulting services. 
The CRIs represented the group of organisation with the highest levels of interaction for both directions (initiating and receiving) and were therefore active experts with an up-to-date knowledge base. They were the key knowledge brokers in the professional field of hydrology. This was an expected result, as the CRIs had such an expert status in environmental research in New Zealand.

In addition, local government represented the key sinks (receivers) and private businesses the key sources (initiators) in this small-world network. Together with CRIs, local government agencies were therefore the key knowledge givers. As a main task of local government in NZ is to provide up-to-date information about the environment to the public, and also to central government, in the form of news and reports, this was also a reasonable result.

As the levels of interaction (by column and by row) were conducted by using valued network data, the mean values represented the average strength of relationships. Therefore, the groups of organisation with the highest mean values, namely the key players - CRIs, local government and private businesses - were also the most strongly connected in the network.

\subsubsection{Case 2 key players}

In comparison to the knowledge transfer structures in Case 1, Case 2 showed a slightly different constellation of key players in its network. The following tables illustrate the mean values and the standard deviations for the levels of interaction in regard to knowledge giving and seeking activities per group of organisation. The highest mean values indicate key sinks in the network.

Table 57: Small-world network 2: Level of interaction by column (key sinks)

\begin{tabular}{|l|c|c|c|c|c|c|c|}
\hline & CRIs & $\begin{array}{l}\text { Central } \\
\text { government }\end{array}$ & Universities & $\begin{array}{l}\text { Private } \\
\text { businesses }\end{array}$ & $\begin{array}{l}\text { Local } \\
\text { government }\end{array}$ & NPOs & Others \\
\hline Mean & $\mathbf{1 9 . 1 7}$ & $\mathbf{1 1 . 8 3}$ & $\mathbf{2 3 . 6 7}$ & $\mathbf{5 . 6 7}$ & $\mathbf{1 6 . 5 0}$ & $\mathbf{0 . 0 0}$ & $\mathbf{0 . 0 0}$ \\
\hline $\begin{array}{l}\text { Std. } \\
\text { Dev. }\end{array}$ & 17.49 & 10.24 & 18.82 & 4.71 & 16.47 & 0.00 & 0.00 \\
\hline
\end{tabular}


Table 57 indicates the universities as the key knowledge givers (key sinks) in the field of marine biology. The second most important knowledge givers were CRIs, then local and central government. Private businesses again showed a low level of knowledge giving activities in the form of advice giving at the workplace, which corresponds to Case 1 results. The group of NPOs and others did not indicate any knowledge giving activities to other organisations in the field.

In contrast, Table 58 reveals two groups of organisation as the key knowledge seekers (key sources): private businesses and central government.

Table 58: Small-world network 2: Level of interaction by row (key sources)

\begin{tabular}{|l|c|c|c|c|c|c|c|}
\hline & CRIs & $\begin{array}{l}\text { Central } \\
\text { government }\end{array}$ & Universities & $\begin{array}{l}\text { Private } \\
\text { businesses }\end{array}$ & $\begin{array}{l}\text { Local } \\
\text { government }\end{array}$ & NPOs & Others \\
\hline Mean & $\mathbf{1 1 . 6 7}$ & $\mathbf{2 3 . 0 0}$ & $\mathbf{1 2 . 6 7}$ & $\mathbf{2 3 . 6 7}$ & $\mathbf{1 . 5 0}$ & $\mathbf{1 . 3 3}$ & $\mathbf{3 . 0 0}$ \\
\hline $\begin{array}{l}\text { Std. } \\
\text { Dev. }\end{array}$ & 13.77 & 19.22 & 10.92 & 18.30 & 1.71 & 2.98 & 2.58 \\
\hline
\end{tabular}

In addition, Table 58 shows few knowledge seeking activities from local government, NPOs and the group of other organisations. Moderate activities were undertaken by universities and CRIs. A relatively high variability (Std. Dev.) particularly for the types with the highest mean and sum values again showed a high diversity in the strength of connections to others.

The following matrix illustrates the three key sinks - universities, CRIs and local government - and two key sources: central government and private businesses. 


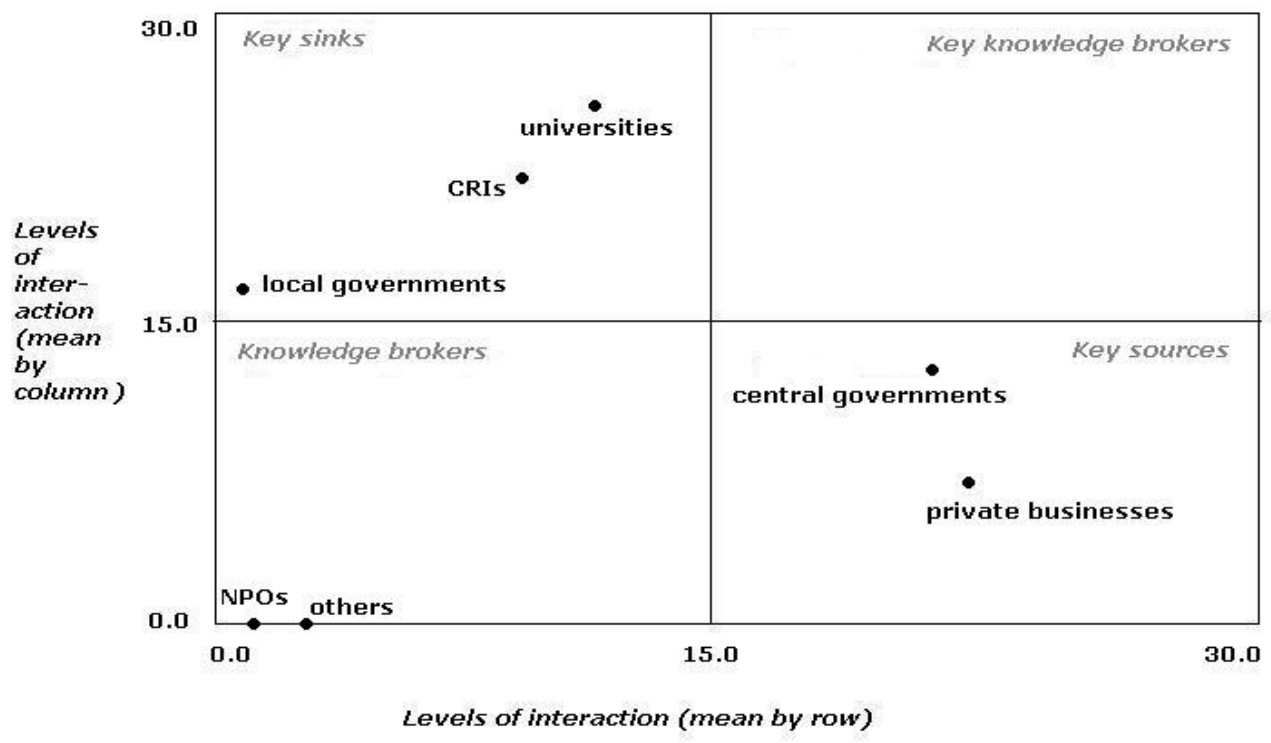

Figure 27: Small-world network 2: Matrix of key sinks and key sources

In Figure 27, no knowledge broker or key knowledge broker could be identified. CRIs did not seek as much as knowledge in the field of marine biology, as they did in the field of hydrology. Moreover, universities showed a higher level of interaction for knowledge giving as well as seeking behaviour than the CRIs. This revealed the universities as the key experts, whereas CRIs and local government came second and third.

Private businesses were again key seekers of knowledge as in Case 1. Unexpectedly, central government agencies also showed a high level of knowledge seeking activities and a moderate level of giving activities. This relatively high activity of central government in the field of marine biology indicated the national significance of this topic and may also be the consequence of government-funded research projects.

The absence of a knowledge broker and the higher number of knowledge givers than seekers indicated a slightly unbalanced knowledge transfer in the network. Nevertheless, this high level of informal advice giving activities may support an effective knowledge exchange. As they had the highest mean and sum values for either knowledge giving or seeking activities, the five identified key players, as 
illustrated in Figure 27, had the strongest connections in the professional field of marine biology.

\subsubsection{Case 3 key players}

Compared to the key players in Cases 1 and 2, the most important groups of organisation in Case 3 showed a different mixture in terms of key sinks, key sources and key knowledge brokers. Table 59 indicates that central and local government were the key sinks in Case 3. Private businesses, CRIs and universities showed moderate values for the mean and sum values in regard to their levels of knowledge giving interaction.

Table 59: Small-world network 3: Level of interaction by column (key sinks)

\begin{tabular}{|l|c|c|c|c|c|c|}
\hline & CRIs & Universities & $\begin{array}{l}\text { Private } \\
\text { businesses }\end{array}$ & $\begin{array}{l}\text { Central } \\
\text { government }\end{array}$ & $\begin{array}{l}\text { Local } \\
\text { government }\end{array}$ & Others \\
\hline Mean & $\mathbf{1 1 . 8 0}$ & $\mathbf{1 3 . 0 0}$ & $\mathbf{1 5 . 2 0}$ & $\mathbf{2 0 . 2 0}$ & $\mathbf{1 9 . 2 0}$ & $\mathbf{0 . 0 0}$ \\
\hline Std. Dev. & 8.795 & 8.149 & 11.754 & 12.608 & 10.068 & 0.00 \\
\hline
\end{tabular}

In contrast to the knowledge giving activities in Table 59, Table 60 shows the level of interaction in regard to knowledge seeking activities. The key sources were local government, then CRIs and private businesses. Universities and the group of other groups of organisation showed the lowest levels of knowledge seeking activities. The variability in regard to the levels of tie strength (as expressed by the standard deviation) was still high, but slightly lower in this case than in Cases 1 and 2.

Table 60: Small-world network 3: Level of interaction by row (key sources)

\begin{tabular}{|l|l|c|l|l|l|c|}
\hline & CRIs & Universities & $\begin{array}{l}\text { Private } \\
\text { businesses }\end{array}$ & $\begin{array}{l}\text { Central } \\
\text { government }\end{array}$ & $\begin{array}{l}\text { Local } \\
\text { government }\end{array}$ & Others \\
\hline Mean & $\mathbf{1 8 . 8 0}$ & $\mathbf{8 . 2 0}$ & $\mathbf{1 7 . 4 0}$ & $\mathbf{1 0 . 8 0}$ & $\mathbf{2 3 . 4 0}$ & $\mathbf{0 . 8 0}$ \\
\hline Std. Dev. & 10.07 & 5.07 & 9.97 & 8.42 & 13.11 & 0.75 \\
\hline
\end{tabular}

This case showed the greatest diversity concerning the distribution of key roles among all three small-world networks. A key sink (central government) and a key source (CRIs) are illustrated in Figure 28. In addition, two key knowledge brokers 
(local government and private businesses) and a knowledge broker (universities) were identified. However, as the levels of interaction for both initiating and receiving relationships were higher for local government, they were strategically better positioned as knowledge brokers compared to the private businesses and universities.

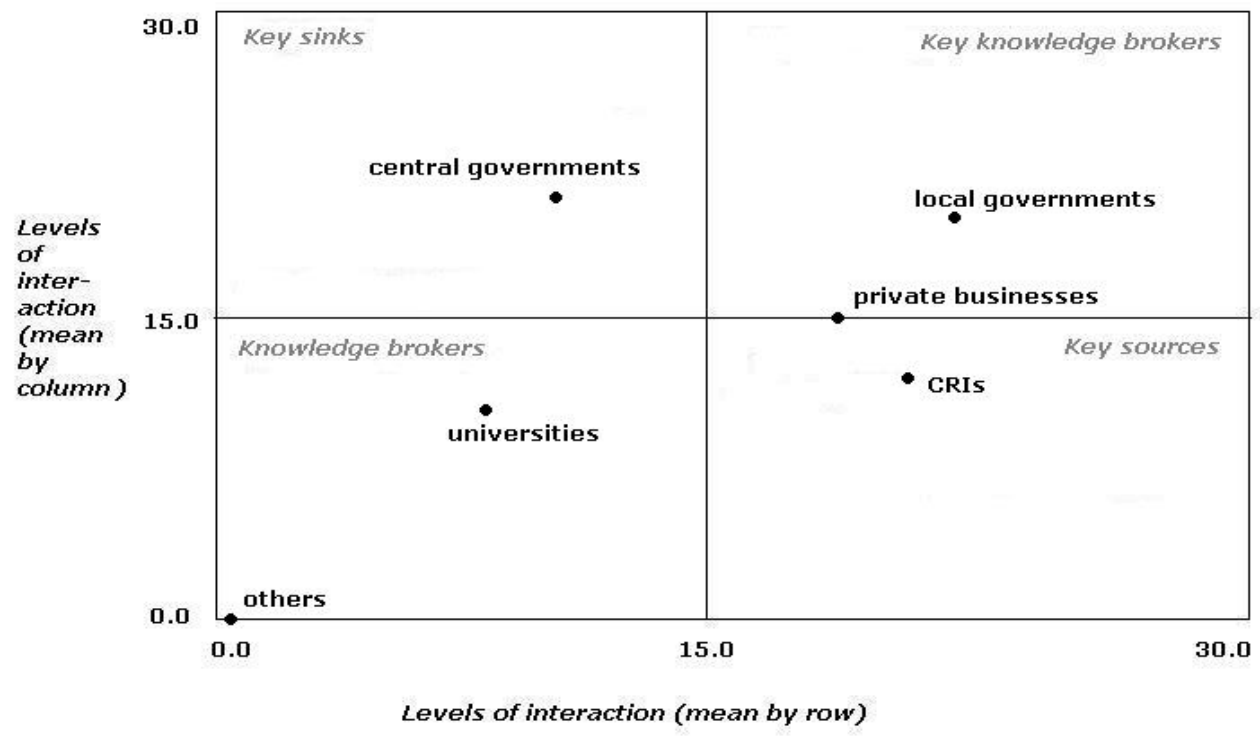

Figure 28: Small-world network 3: Matrix of key sinks and key sources

As illustrated in Figure 28, the CRIs were the key initiators of relationships and therefore key seekers of knowledge. This is a rather surprising result, particularly compared to Cases 1 and 2, in which CRIs showed a much higher level of knowledge giving activities. Although CRIs were more focussed on seeking knowledge in this case, they still had a moderately high level of knowledge giving activities. Nevertheless, local government agencies took over the role of the key knowledge brokers in this case. Local government therefore represented the experts with the most up-to-date knowledge base in this field of coastal hazards.

Unexpectedly, central government agencies were the key givers of knowledge. This may have been due to their high interactions in terms of providing regulations and policies to other groups of organisation in the area of coastal hazards. Private businesses also showed a strong position in Figure 28. Together with the universities 
and local government, the private businesses therefore indicated a balanced level of interactions for knowledge giving as well as seeking activities. They were both active contributors to the network's knowledge exchange. The four key groups of organisation however - local government, private businesses, central government and CRIs - were the most strongly connected ones in the coastal hazards network due to their high level of interaction values.

Summing up, the first two key roles - key sinks and key sources - were revealed in each of the three small-world networks. Key knowledge brokers could only be identified in Cases 1 and 3. These key knowledge brokers, which represented the most up-to-date knowledge bases in the networks, were CRIs for Case 1 (hydrology) and local government and private businesses for Case 3 (coastal hazards). In addition, the key roles in each case were the most strongly connected in the networks.

Alongside these three key roles, the most influential key players throughout all cases according to Information Centrality are outlined in the following paragraphs.

\subsubsection{Most influential key players (Information Centrality scores)}

In addition to the key sinks, key sources and key knowledge brokers described, a number of other key players were identified in each of the three networks. These key players could be determined by identifying the groups of organisation with the highest Information Centrality scores. Due to this study's network characteristics (a high number of short paths and different levels of tie strength among network members) and the types of knowledge mostly transferred (replicable knowledge: electronically available and tacit), Information Centrality was selected as the most

appropriate centrality measure for these types of knowledge networks ${ }^{14}$. A table in Appendix 24 shows the Information Centrality score for each group of organisation

$14 \quad$ See Chapters 4-6 
per case. The most central groups of organisation throughout all three small-world networks were:

Table 61: Sum of Information Centrality scores per group of organisation

\begin{tabular}{|c|c|}
\hline Group of organisation & Sum of Information Centrality scores over all cases \\
\hline CRIs & 72.45 \\
\hline Private businesses & 71.43 \\
\hline Local government & 70.04 \\
\hline Central government & 66.27 \\
\hline Universities & 56.07 \\
\hline
\end{tabular}

All five groups of organisation in Table 61 show a high Information Centrality score. However, the three more powerful groups of organisation, which are CRIs, private businesses and local government, may have a reasonable influence on ICT decisions and educational offerings in the network. As might have been expected, these groups of organisation were also identified as key roles (key sinks, key sources or key knowledge brokers) throughout all cases when investigating the levels of interaction.

These three key players - CRIs, private businesses and local government represented a mixture of groups of organisation with different kinds of ownership: public (local government, CRIs) and private (private businesses). In this regard, these small-world networks reflected a good power balance among heterogeneous organisations in the professional fields of hydrology, marine biology and coastal hazards.

\subsubsection{Summary of key players}

This cross-case analysis of the levels of interaction helped reveal a number of key roles (key sinks, key sources and key knowledge brokers) and the most influential groups of organisation by their Information Centrality in the networks. This analysis also indicated a relatively good balance of knowledge transfer among these key 
players in each of the three small-world networks. Knowledge exchange may benefit from these balanced structures, particularly as these key players from different groups of organisation were working in the same field, but from diverse perspectives.

Two of the three small-world networks in this study revealed at least one important knowledge brokering role that is represented by a specific group of organisation: CRIs in Case 1 and local government agencies and private businesses in Case 3. These appeared as the groups of organisation with the most up-to-date knowledge in their respective fields of environmental research. Table 62 summarises the key players as defined by their levels of interaction for each network.

Table 62: Summary of key players in the three small-world networks

\begin{tabular}{|l|l|l|l|}
\hline Key role/Case & \multicolumn{1}{|c|}{ Case 1 } & \multicolumn{1}{c|}{ Case 2 } & \multicolumn{1}{c|}{ Case 3 } \\
\hline Key sinks & Local government & $\begin{array}{l}\text { Universities, CRIs, local } \\
\text { government }\end{array}$ & Central government \\
\hline Key sources & Private businesses & $\begin{array}{l}\text { Central government, } \\
\text { private businesses }\end{array}$ & CRIs \\
\hline $\begin{array}{l}\text { Key knowledge } \\
\text { brokers }\end{array}$ & CRIs & - & $\begin{array}{l}\text { Local government, } \\
\text { private businesses }\end{array}$ \\
\hline
\end{tabular}

Three groups of organisation were identified by this cross-case analysis as the same three key players throughout all three small-world networks:

- CRIs

- local government

- private businesses

These three groups were present in all three networks as either key sinks, key sources or key knowledge brokers. Moreover, CRIs, local government and private businesses were identified, with the help of the Information Centrality measure, as the most influential groups of organisation throughout all three studies. This confirmed the results regarding the levels of interaction analysis. In addition, these results showed these key types' expert status in the environmental R\&D sector in New Zealand. 
CRIs were in an expected position as research experts in the field, whereas local government agencies and private businesses showed surprisingly strong expertise too. The strong connections among these three groups of organisation revealed a good base for knowledge transfer. They may have also co-ordinated the transfer of knowledge in the network.

This section examined the levels of interaction in regard to the knowledge transfer activities of the groups of organisations that participated in this study. Alongside these levels, this study also looked at the Information Centrality scores for the types or organisation throughout all three cases. This helped identify a number of key players for each network and in the overall investigated field of environmental research. These were CRIs, local government and private businesses.

In addition, this social network represents a decentralised network, as there was no single most central organisation in the network despite there being a group of central organisations. Thus the centrality measures and additional interpretations of the level of interaction helped confirm the ontological structure of this knowledge network as a small-world network structure.

The following section describes the types of knowledge most often transferred in the three small-world networks.

\subsection{Types of knowledge transferred}

\subsubsection{Introduction}

This section outlines the most important types of knowledge transferred among all types of ties in the three small-world networks. Together with knowledge transfer, knowledge characteristics were investigated in several studies (e.g. Amin \& 
Cohendet, 2004; Sammarra \& Biggiero, 2008). These studies were for example looking at characteristics such as tacit-codified, individual-social, appropriableexclusive, divisible-indivisible and subject-specific types (technological-managerialmarket knowledge). This study differentiated knowledge according to a variety of aspects which are listed below as a reminder:

- $\quad$ electronic availability (available, unavailable)

- $\quad$ formality (formal, informal)

- $\quad$ codifiability (non-codified/tacit, explicit)

- $\quad$ description (how-to, factual)

- $\quad$ meta-knowledge (references to other sources, contact details of experts)

In terms of codifiability, previous literature in the field investigated the types of knowledge transferred (particularly tacit and codified) by weak and strong ties (e.g. Granovetter, 1973; Hansen, 1999; Reagans \& McEvily, 2003). Although Hansen (1999; 2002) identified that strong ties could transfer tacit knowledge more easily than weak ties, no significant differences between strong and weak ties were revealed for the transfer of tacit knowledge in the cross-case analysis of this study.

Moreover, non-codified/tacit knowledge, in general, was the type of knowledge that was transferred less often compared to codified data (electronically available). This could be identified for both knowledge transfer processes: advice giving and seeking activities. Nevertheless, informal how-to knowledge and informal factual knowledge were transferred more often in a non-codified/tacit form rather than in their electronically available codified form. This was identified especially for the advice giving processes by both weak and strong ties. Supporting data for Cases 2 and 3 that particularly considered non-codified/tacit knowledge are found in Appendix 25.

Strong and intermediate ties were well embedded in this study's small-world networks. This put them in a favoured position in regard to the transfer of all types of knowledge. In addition, no differences between strong and weak ties in relation to the 
transfer of non-codified/tacit knowledge could be identified in this study. According to Reagans and McEvily's (2003) investigation of network structure and knowledge transfer, there is also rather weak evidence for strong ties facilitating the transfer of tacit knowledge. Moreover, they suggest "that it is easier to transfer all kinds of knowledge in a strong tie and more difficult to transfer all kinds of knowledge in a weak tie" (Reagans \& McEvily, 2003, p.262). In addition, they argue that strong ties which are embedded in a dense web of third party relationships can transfer all types of knowledge better.

Apart from the codifiability of knowledge, electronically available and unavailable knowledge was particularly important to this study. In all three cases, electronically available knowledge was the most important, then non-codified/tacit knowledge and then electronically unavailable but codified knowledge. The following paragraphs expand on these results.

\subsubsection{Electronically available types of knowledge}

The most important electronically available types of knowledge for study participants per case were:

Table 63: Electronically available types of knowledge most important for transfer per case

\begin{tabular}{|l|l|}
\hline Case & $\begin{array}{l}\text { Electronically available types of knowledge most important for } \\
\text { transfer }\end{array}$ \\
\hline Case 1 & Formal factual knowledge, references to other sources of knowledge \\
\hline Case 2 & Formal (factual and how-to) knowledge, meta-knowledge (both subtypes) \\
\hline Case 3 & $\begin{array}{l}\text { Meta-knowledge (both subtypes), formal factual knowledge, informal how- } \\
\text { to knowledge }\end{array}$ \\
\hline
\end{tabular}

Formal factual knowledge and references to other sources of knowledge (metaknowledge) were the most important types of knowledge transferred in all three small-world networks. Examples of formal factual knowledge were research papers in 
pdf format, reports, journal papers, manuscripts, web sites, images, published conference papers, data archives (time series) and knowledge about the use of technical equipment.

The electronic media used to transfer this knowledge was predominantly email and more rarely discussion forums and Skype. Due to a lack of sufficient bandwidth, it was difficult to transfer large amounts of data.

\subsubsection{Electronically unavailable types of knowledge}

As this study aimed to develop implications for ICT support for this interorganisational knowledge transfer, the types of knowledge that were electronically unavailable at the time of this study were particularly important to the researcher, as ICT support might be more urgently needed for these cases. In particular, the most important electronically unavailable types of knowledge for study participants per case are shown in Table 64.

Table 64: Electronically unavailable types of knowledge most important for transfer per case

\begin{tabular}{|c|l|}
\hline Case & \multicolumn{1}{|c|}{$\begin{array}{c}\text { Electronically unavailable types of knowledge most important for } \\
\text { transfer }\end{array}$} \\
\hline Case 1 & $\begin{array}{l}\text { Informal how-to knowledge, meta-knowledge (both subtypes), informal } \\
\text { factual knowledge }\end{array}$ \\
\hline Case 2 & $\begin{array}{l}\text { Non-codified/tacit informal how-to knowledge, non-codified informal } \\
\text { factual knowledge }\end{array}$ \\
\hline Case 3 & $\begin{array}{l}\text { Codified references (meta-knowledge), non-codified meta-knowledge } \\
\text { (both subtypes), non-codified informal factual knowledge }\end{array}$ \\
\hline
\end{tabular}

For all three cases, (non-codified) informal factual knowledge appeared as an important type of knowledge. In addition, (non-codified) meta-knowledge (both subtypes) and (non-codified/tacit) informal how-to knowledge were only important in two of the three cases. Examples of these types were experiences, opinions and suggestions as well as additional research material that has not been written down or 
published. These types of knowledge were transferred mostly by phone or in face-toface meetings.

In summary, the more important electronically available types of knowledge throughout all cases were formal factual knowledge as well as references to other sources of knowledge (meta-knowledge). The most important electronically unavailable type of knowledge was non-codified informal factual knowledge. Besides these specific characteristics of the types of knowledge, ICT support for knowledge transfer should also consider the users' preferred media characteristics. This consideration might help enhance the user acceptance and actual use of the recommended ICT. Therefore, the following section outlines the cross-case analysis results for the most preferred media characteristics.

\subsection{Media/ICT use for knowledge transfer}

In this section, key results for the media/ICT used and study participants' preferred media characteristics for knowledge transfer and preferred ICT-based knowledge services for learning are outlined. In this regard, this cross-case analysis shows similar patterns among the three cases. These key results are described in the context of inter-organisational knowledge transfer (advice giving and seeking activities) and learning in the workplace.

\subsubsection{Media/ICT use}

This section outlines key results of study participants' media/ICT use. They could either select a given option or provide their own answers for their media/ICT use. The media/ICT options provided to study participants were email, telephone, (face-toface) meeting, workshops/seminars/conferences, video-conferencing, online 
discussion forums, and wikis or blogs. This presents a combination of media previously used for similar research (Haythornthwaite 1999) plus current social software (wiki and blog). Workshops/seminars/conferences represented an additional group of face-to-face group communication media that related to the knowledge transfer and learning context of this study. As there were no considerable differences between wiki and blog usage, they were aggregated and renamed social software for the analysis. All these described options relate to media/ICT use at the individual and group level, thus representing tools that allow people to collaborate with each other.

The following list demonstrates the media use per case. The more important media are listed at first.

- Case 1: Email, telephone, face-to-face, workshop/seminar, video-conferencing

- Case 2: Email, telephone, meeting, workshop/seminar/conference, videoconferencing, online discussion forum

- Case 3: Email, telephone, meeting, workshop/seminar/conference, online discussion forum, video-conferencing, social software (blog)

The medium predominantly used to transfer knowledge in the three small-world networks was email. This is an interesting aspect considering the issue of email overload. Although issues were reported in terms of receiving too many emails, email was still the most commonly accepted and important medium for knowledge transfer in the small-world networks.

Other traditional media, such as face-to-face meetings, including workshops/seminars/conferences, and telephone calls, followed. Relatively new ICTbased media such as discussion forums, video-conferencing and social software ranked third and were rarely used. Supporting data can be found in the Appendices 26-28 for all three cases.

This study was also looking at key differences of media use among more strongly or more weakly tied groups of organisations. The main differences were related to the 258 
use of video conferencing and social software. Compared to study participants in weak relationships, participants in strong relationships used video-conferencing a few times to follow up on initial contacts (Case 1). Supporting data are attached in Appendices 29-31. This may be due to the fact that only the key organisations which were connected strongly were able to participate in the KAREN network, which provides a high-capacity and high-speed network for education, science and research in New Zealand. These key organisations were therefore able to use appropriate bandwidth for video-conferencing. In addition, the use of social software also appeared to be slightly stronger in strong rather than weak relationships.

Moreover, social software was used more by younger study participants. An explanation for the preferred use of social software for relatively new and inexperienced employees might be the fact that they are typically younger in age than senior staff; and social software is the type of communication medium used more often by the digitally-savvy generation born between 1980 and 1999 (Kupperschmidt, 2001). In the future, this younger generation will likely use more social software for informal knowledge transfer in advice giving and seeking activities in the workplace. However, social software represents a collaboration tool at the individual and group level. For the use of social software at an inter-organisational level in the context of this study, the respective software would need to be hosted externally, and not within any one of the key organisations. It became apparent that key public organisations, such as CRIs and government departments, had to follow strict IT security guidelines and would therefore not be able to open up an intranet to external users.

\subsubsection{Media characteristics}

This section outlines study participants' preferred media characteristics for advice giving and seeking activities. The employed media's characteristics and the key results are described in relation to Media Synchronicity Theory ${ }^{15}$ (MST) (Dennis et

${ }^{15}$ Section 2.5 .2 
al., 2008). MST relates to media capabilities and aims to identify the best possible medium to support users and their communication processes within a specific social context (Dennis et al., 2008). Media synchronicity was defined as "the extent to which the capabilities of a communication medium enable individuals to achieve synchronicity", which is "a state in which individuals are working together at the same time with a common focus" (Dennis et al., 2008, p.581).

This study included the following media characteristics:

- $\quad$ quick transfer of information

- $\quad$ ease of use

- $\quad$ secure communication (data transfer)

- $\quad$ ability to edit information before transfer

- $\quad$ ability to use multiple ways of communication with text, voice and/or pictures

- $\quad$ ability to save information for later reuse

Four of these six media characteristics were directly related to characteristics used in MST: the ability to edit information before transfer, the ability to use multiple ways of communication with text, voice and/or pictures, the ability to save information for later reuse and quick transfer of knowledge (transmission velocity). The six media characteristics are now explained.

Quick transfer of information has been defined as a media characteristic that allows the knowledge giver to quickly transfer knowledge, and, in turn, the knowledge seeker to benefit from a quick response. Therefore, in relation to MST, quick transfer of information represents transmission velocity. Transmission velocity describes a medium's speed of delivering a message to the recipient (e.g. the knowledge seeker) (Dennis et al., 2008). Dependent on the medium and the use of this medium, transmission velocity has high (e.g. video-conferencing), medium (e.g. instant messaging) or low synchronicity (e.g. documents) (Dennis et al., 2008). In the 
context of this study, media with a high transmission velocity might best support knowledge seekers who are interested in receiving knowledge quickly.

Ease of use refers to a medium's high degree of intuitiveness in terms of usage. The ease of use does not only imply that the medium is intuitive to use, but also that the user knows about its existence and knows how to use it. The integration of a medium for specific tasks in a communication process may facilitate the ease of use. Email was easy to use for most study participants.

Secure communication (data transfer) has been added as a media characteristic, as it appeared important in ensuring a safe environment for transferring interorganisational knowledge, particularly in regard to situations in which business partners not only collaborate on certain projects, but also compete on other projects at the same time. This may include the application of appropriate firewalls in the enterprise-wide IT infrastructure.

The ability to edit information before transfer gives the user the freedom to properly prepare or correct a message before sending it. A knowledge giver may want to add another explanation or change an example before sending it to the knowledge seeker. Several ICT-based media support this feature, for example email, discussion forums and social software. Other media such as video-conferencing tools or the telephone cannot provide this feature. This media characteristic has also been applied in MST.

The ability to use multiple ways of communication with text, voice and/or pictures allows users to express themselves in a variety of ways which are not limited to text. These features are supported by forms of multimedia or face-to-face communication. Examples could be video-conferencing and face-to-face meetings. This media characteristic has also been applied in MST.

The ability to save information for later reuse enables the user to save information, for example an email, a voice message or discussion forum thread. This is a 
particularly important characteristic in the context of knowledge reuse. A knowledge seeker may want to save knowledge received earlier in order to come back to it at a later stage or to provide this knowledge to another seeker. This media characteristic is also applied in MST.

Media such as email, video-conferencing or instant-messaging show diverse levels of synchronicity. For example, a video-conference tool allows for a synchronous and quick transfer of knowledge (transmission velocity) and therefore has a high synchronicity (Dennis et al., 2008). In contrast, email represents an asynchronous medium for transferring knowledge, and its transmission velocity was rated low to medium. This reflects a low level of synchronicity (Dennis et al., 2008). To support both low and high synchronicity for achieving an effective communication process, Dennis et al. (2008) recommend a mix of diverse media.

The following list demonstrates study participants' preferred media characteristics per case. The more important characteristics are listed at first.

- Case 1: ease of use, ability to save information for later reuse, quick transfer of information

- Case 2: ease of use, ability to save information for later reuse, quick transfer of information and ability to use multiple ways of communicating

- Case 3: ease of use, ability to save information for later reuse, quick transfer of information and ability to use multiple ways of communicating

Overall, the media characteristics most preferred by knowledge givers and knowledge seekers, regardless of specific tie strength, were

- $\quad$ ease of use

- quick transfer of information

- ability to save information for later reuse 
Supporting data from all three cases are attached in the Appendices 32-34. Two of these three media characteristics, quick transfer of information and ability to save information for later reuse, directly related to MST (Dennis et al., 2008). Media Synchronicity Theory (MST) was employed as an informing theory for this study.

In regard to the more preferred media characteristic 'ability to save information for later reuse', e-knowledge seekers may want to save knowledge that they received earlier in order to come back to it at a later stage. Moreover, a knowledge seeker may want to reuse knowledge in another context, which implies a reasonably good understanding of this knowledge.

In MST, ability to save information for later reuse is referred to as reprocessability. Dependent on the medium and its use, reprocessability has high (e.g. email, electronic conferencing), medium (e.g. instant messaging) or low synchronicity (e.g. videoconferencing). In the context of this study, media with a high reprocessability might support knowledge seekers and givers alike. Seekers could save knowledge for reexamination whereas givers would not need to provide the same advice twice.

In summary, quick transfer of information and ability to save information for later reuse were two diverse but important media characteristics in this context of interorganisational knowledge transfer. The relation to MST showed that these characteristics have diverse degrees of synchronicity to assist an effective communication process, for example advice giving and seeking activities at the workplace.

This section outlined key results of the cross-case analysis in regard to study participants' preferred media characteristics. In relation to these characteristics, Dennis et al.'s (2008) recommendations on media use, which represent collaborative tools, thus media/ICT at the individual and group level, will provide some solid background information from which to draw implications for ICT-based media support (Chapter 8). In addition, this background information will be supported by 
the following section that outlines the preferred knowledge services for learning in relation to Wenger and Ferguson's (2006) knowledge ecology model.

\subsubsection{Knowledge services for learning}

This section reports on study participants' preferred ICT-based knowledge services for learning in addition to currently provided face-to-face workshops and seminars in their professional fields. Study participants were asked to select their individually preferred learning methods and characteristics.

In Cases 2 and 3, study participants could select their most preferred ICT-based knowledge services for learning from the following four options: e-book, online discussion forum and chat, online learning, and/or online interaction with experts. This pre-selection of services was drawn from Wenger and Ferguson's (2006) knowledge ecology model. This model is described in more detail in Section 2.5. of the literature review. Initially, Case 1 presented more options that additionally included blended learning, e-mentors and a learning community. However, for Cases 2 and 3 , the options were reduced to the four services relatively well-known by study participants.

In addition, the four options represented the four different groups of the knowledge services model: exploration, information, participation and guidance. These four groups were explained in detail in the literature review chapter ${ }^{16}$. The following list outlines which knowledge service supports which main characteristic in the model:

e-book (supports exploration purposes)

online discussion forum and chat (supports participation purposes)

$>$ online learning (supports informing purposes)

$>$ online interaction with experts (supports guiding needs)

\footnotetext{
${ }^{16}$ Section 2.5.3.
} 
The following list indicates study participants' preferred knowledge services per case. The more important services are listed at first.

- Case 1: Interaction with experts, blended learning (incl. online learning), discussion forum

- Case 2: Online interaction with experts, online learning, online discussion forums and chats, e-book

- Case 3: Online interaction with experts, online discussion forums and chats, online learning, e-book

In all three cases, the preferred knowledge service was online interaction with experts. Supporting data for all three cases are attached in Appendices 35, 36 and 38. The following figure illustrates the results from Cases 2 and 3, as study participants in these cases could select their most preferred ICT-based knowledge services for learning from the same four options: online interaction with experts, online learning, online discussion forums and chats and e-book.

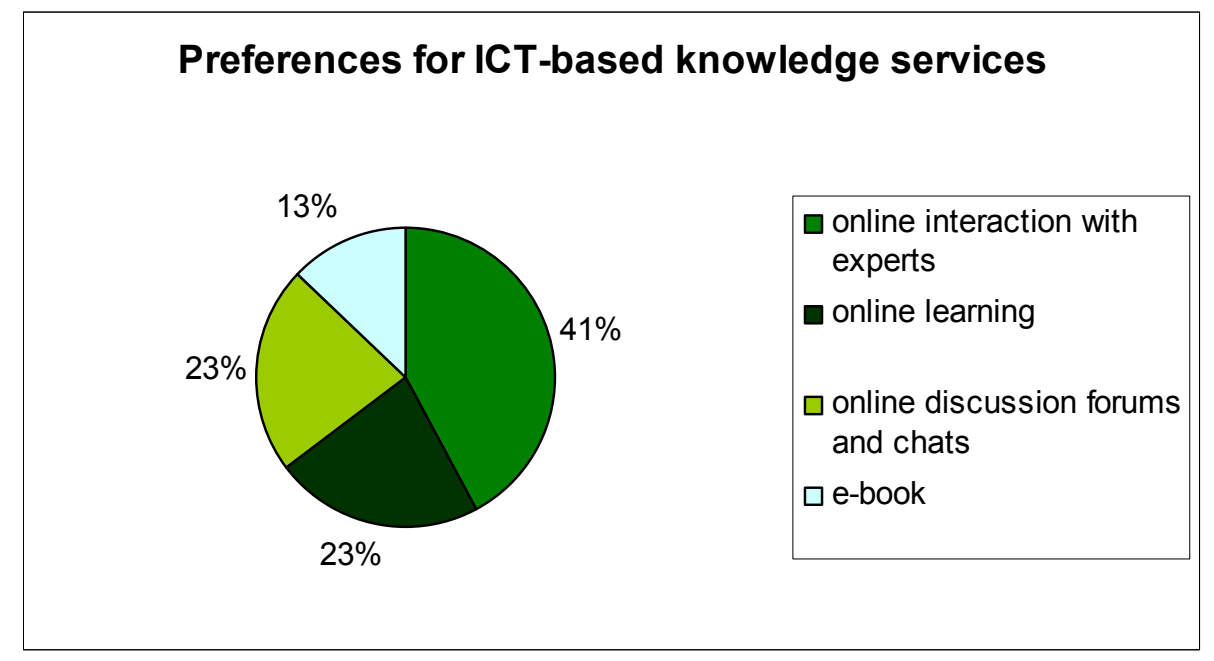

Figure 29: Preferred ICT-based knowledge services

The pie chart in Figure 29 presents the percentage of each knowledge service calculated by dividing the number of responses per service by the number of 
responses for all services in Cases 2 and 3. Online interaction with experts was first with 41 per cent, followed by online learning and online discussion forums and chats (both 23 per cent) and finally e-book with 13 per cent.

Online interaction with experts was the most preferred knowledge service throughout all three cases independent of professional domain, job experience levels, levels of tie strength and type of organisation. It was also preferred equally by male and female study participants. In the context of this study, most experts in the field were typically employed at the more central and powerful groups of organisations. Therefore, key players, such as CRIs, locals government agencies and private businesses, as identified in Section 7.3, may also be providers of the knowledge service 'online interaction with experts' to other groups of organisations in the network. This could for example be realised by introducing interactive online seminars.

Interaction with experts can be viewed from diverse perspectives and may incorporate different learning characteristics and benefits, such as guidance, information and participation. In Wenger and Ferguson's (2006) model, interaction with experts is not particularly connected to an online medium, as their aim was to provide a range of knowledge services for learning independent of a specific medium or mechanism. However, interaction with experts may have a variety of learning characteristics such as guiding or informing the learner, or providing the possibility for the learner to engage in a discussion with an expert.

In Wenger and Ferguson's (2006) knowledge ecology model, interaction with experts was suggested primarily as a service that connects people for a guided learning experience which allows both expert and learner "to apply their knowledge and skills to new situations in a realistic environment" (p.88).

However, this study's network members who selected this service as their most preferred knowledge service also indicated that they would prefer an informing or participating knowledge service for learning rather than a guiding service. These 
results became particularly apparent in Cases 2 and 3. Supporting data can be found in Appendices 37 and 39.

Wenger and Ferguson (2006) describe the guiding service as an extension to an informing service provided by experts. As expert and learner are applying new knowledge together in an on-the-job situation, there is also a degree of participation involved, even if it is not the level of participation inherent to communities. Therefore, the guiding service comprises informing and participating services to some degree (Wenger \& Ferguson, 2006).

However, learning characteristics may be influenced by the knowledge transfer context and the degree of formality in regard to knowledge services for learning. For example, a formal learning initiative, such as a seminar, conference or workshop, would most often contain more informing and guiding rather than participating characteristics. In contrast to guidance and information, however, the participation characteristic appears particularly crucial for advice giving and seeking activities in the networks. It allows for real interaction with experts. Therefore, a more informal learning initiative might facilitate this participation.

In summary, this section revealed that the knowledge service of online interaction with experts can include diverse learning characteristics: guidance, information and participation. Although a guiding service, as suggested by Wenger and Ferguson (2006), implies facilitation by a dominant teacher (an expert in this case), interaction with experts may also imply the participation of knowledge seekers or other additional knowledge givers (besides the facilitating expert). Both the guiding as well as the participating service may have additional informing characteristics.

Overall, the identification of online interaction with experts as the most preferred knowledge service for additional learning will help recommend appropriate ICT for learning in the workplace. Interaction with experts could be facilitated by different 
ICT-based media that are collaborative in nature and thus support advice giving and seeking activities. These recommendations will be outlined in the final chapter.

\subsubsection{Summary}

This section described key results in regard to the media/ICT used and study participants' preferred media characteristics for knowledge transfer and preferred ICT-based knowledge services for learning. Email was the most preferred medium for advice giving and seeking activities. Study participants' preferred media characteristics were ease of use, quick transfer of information and ability to save information for later reuse. These preferences were confirmed by study participants in Phase 3. However, a specific ranking of the preferred media characteristics in regard to the survey results (Phase 2) could not be confirmed by every study participant:

"It is nice to have a reference, but I rank all three characteristics equally - if an information source is not easy or quickly accessible I will not use it. I just don't have the time." (Participant 2)

The most preferred ICT-based knowledge service for learning was online interaction with experts.

These key results were described in the context of inter-organisational knowledge transfer (advice giving and seeking activities) and learning in the workplace. Implications for ICT support, which are presented in the next chapter, are based on these key results together with key findings in regard to structural properties, such as the network topology, that were presented earlier in this chapter.

The following section summarises and discusses this cross-case analysis chapter. 


\subsection{Conclusion and discussion}

\section{Summary}

This chapter described and interpreted the results of this study's cross-case analysis in order to answer the first two sub-questions of this thesis:

1) What are the key structural properties of advice networks and their implications for knowledge transfer?

2) Which types of knowledge are transferred in the advice networks?

The overarching goal was to identify common patterns among the three cases to present reliable results. This included an interpretation of the findings regarding the knowledge transfer context.

One key result of this cross-case analysis was the identification of three small-world networks. A small-world network represents a decentralised ontological network structure that includes some degree of local clustering. In this regard, this chapter described, compared and illustrated the characteristics of the three social networks in this study including the high clustering coefficients and the high number of shortest paths.

Moreover, an even more important key finding could be established by the definition and identification of intermediate ties in social networks. In particular, the definition of intermediate ties when considering more than one indicator for tie strength (in inter-organisational social networks) was crucial. In particular, the k-means clustering in combination with a subsequent investigation of the levels of reciprocity was suggested. The k-means clustering initially grouped all total tie strength values in a network into three groups: weak, intermediate and strong ties. Then, an investigation of the levels of reciprocity at the borderlines of this initially identified group of intermediate ties was carried out. 
The important roles of intermediate ties in social networks were also pointed out. Besides their numerous appearances in this study's social networks, the intermediate ties represented two specific roles. Their significance was represented by either Role 1, which was linking otherwise weakly linked groups of organisations to the network, or Role 2, which was linking two otherwise very strongly connected groups of organisations (key players). Therefore, intermediate ties might help receive new knowledge from outside the network with the help of Role 1 ties and also help access the local networks of other strongly connected network members with the help of Role 2 ties.

In addition, by comparing outcomes of the analysis on the levels of interaction in all three cases, three key players could be singled out: CRIs, local government and private businesses. The diversity in terms of the ownership of these organisation groups shows a balanced power constellation among public and private organisations in the networks. The resulting balanced knowledge transfer was confirmed by the constellation of key sinks and key sources, as well as key knowledge brokers. The key players in Cases 1 and 3 - CRIs, local government and/or private businesses also played the role of knowledge brokers.

Moreover, the types of knowledge transferred most frequently within all three cases were investigated. The most important electronically available types of knowledge, throughout all cases, were formal factual knowledge as well as references to other sources of knowledge (meta-knowledge). Formal factual knowledge could represent published research material, whereas other sources of knowledge represent metaknowledge and could be electronically available in the form of literature references in a database. The most important electronically unavailable type of knowledge was non-codified informal factual knowledge. This type could represent additional research material that has not been written down or published.

The predominant medium used in all three networks was email. This was followed by other traditional media, such as face-to-face meetings and telephone calls. Relatively 
new ICT-based media such as discussion forums, video-conferencing and social software ranked third and were rarely used. In addition, the cross-case analysis also helped gain knowledge on preferred media characteristics and ICT-based knowledge services of study participants from all three cases.

Study participants' preferred media characteristics were quick transfer of knowledge (transmission velocity), ease of use and the ability to save information for later reuse (reprocessability). Two of these three characteristics - transmission velocity and reprocessability - are also employed in Media Synchronicity Theory (Dennis et al., 2008) which also informed some implications on ICT support in the following chapter.

Finally, the most preferred ICT-based knowledge service for learning at the workplace in all three networks was online interaction with experts. In this regard, study participants were not only interested in guiding and informing knowledge services for learning, but also in participatory ICT-based learning initiatives such as discussion forums and chats or instant messaging. Wenger and Ferguson's (2006) knowledge ecology model informed these results on knowledge services and their characteristics.

Together with the key results on the structural properties of the social networks, study participants' preferred media characteristics and ICT-based knowledge services for learning helped provide a basis for answering the third sub-question:

How can ICT support these knowledge transfer structures and processes?

The next chapter outlines these implications for ICT support. The following paragraphs discuss this chapter's key results in the context of current literature as well as from a practitioner's point of view. 


\section{Discussion of key results}

This thesis identified small-world network structures for all three cases, due to their large number of short paths and their high cluster coefficients. The identification of all three social networks as small-world networks presents some important aspects for the inter-organisational context, for example the advantage of having relatively easy access to inter-organisational knowledge sources within the networks. Moreover, small-world networks were considered the ideal network structure to facilitate knowledge transfer and creation (e.g. Cowan, 2004; Cowan \& Jonard, 2004).

Other literature suggests similar results (Schilling \& Phelps, 2007), particularly in regard to knowledge creation in small-world networks. According to Schilling and Phelps (2007), a small-world network structure is the ideal structure to optimise the output (knowledge creation) of inter-firm knowledge networks such as alliances, as these structures allow for the right bandwidth (strength) and the appropriate reach (connectivity) among network members. Key players, such as CRIs, local governments and private businesses, could lead the formation of formal networks such as alliances to foster project outputs and compete internationally.

The small-network topology is a key ontological structure, and ICT can efficiently support information processing in these networks (Kwon et al., 2007). In particular, ICT could support routine communications among network members (Kwon et al., 2007). Routine communications may imply the transfer of codified knowledge, which was mainly published research material und literature references in this study. However, besides the transfer of codified knowledge, the aim of this study was to also look at potential ICT support for transferring thus-far electronically unavailable knowledge, which was non-codified (informal factual) knowledge in the form of unpublished research material in this study context.

In addition, ICT allows for connections across different organisations, and these connections help encourage collaboration and knowledge transfer among network 
members (Kwon et al., 2007). This is particularly important for remotely located organisations or heterogeneous groups of organisations (Baum, Rowley \& Shipilov, 2004). In the context of this study, remotely located organisations were, for example, represented by a few remote regional councils. Heterogeneous groups of organisations included CRIs, universities, local government and private businesses in this inter-organisational context.

Although Kwon et al. (2007) suggest a random network structure for knowledgeintensive industries such as the R\&D sector, this study's findings revealed smallworld structures in all three networks. Both topologies - random and small-world are decentralised network structures. Kwon et al.'s (2007) argument for a purely decentralised random structure relies on information regarding social networks within organisations. Employees within organisations in a knowledge-intensive environment often communicate laterally, work with a similar amount of information, and also show a similar share of responsibility (Kwon et al, 2007).

Such a purely decentralised organisational environment was not represented by the inter-organisational context of this study, which instead revealed some degree of local clustering, particularly among CRIs, private businesses and local governments, within the networks. Therefore, the small-world network structure appeared more relevant which confirmed the findings of the clustering coefficient analysis and the short path considerations. Drawing on this study's results, the identification of small-world network structures for inter-organisational social networks in the R\&D sector shows promising aspects for knowledge transfer that could be supported by ICT.

In relation to recent research in inter-organisational knowledge transfer (EasterbySmith et al., 2008), the role of boundaries were identified as a key theme for future research. This includes social relationships between organisations that may inhibit or facilitate knowledge transfer. Due to their important roles identified in this thesis, intermediate ties in social networks therefore represent a specific type of relationship that facilitates inter-organisational knowledge transfer. 
In particular, intermediate ties in the form of 'Role 1 ties', as described in Section 7.2.4, have a key role for knowledge transfer and creation. These intermediate strong ties connect otherwise weakly linked groups of organisation and could thus act as scouts to help integrate new knowledge from external sources outside the network. In addition, 'Role 1 ties' could also act as liaisons to help maintain important long-term relationships.

Although previous literature identified intermediate ties (e.g. Dodds et al., 2003; Granovetter, 1973; Onnela et al., 2007), these studies focussed on social relationships at the individual level and neglected the importance of these intermediate ties. Nevertheless, Dodds et al.'s (2003) results strengthened the significance of intermediate ties, as their investigations revealed that these ties rather than weak ties are used for advice seeking activities.

There has also been a lack of research to properly categorise weak, intermediate and strong ties in social networks, particularly when they have more indicators for tie strength. Petróczi et al. (2007), who had several indicators for tie strength, made a contribution in this regard. They classified all ties into the three categories - weak, intermediate and strong - by employing k-means clustering. Their research explored tie strength within an organisational, but not inter-organisational, context. Further, they did not use the level of reciprocity as a second criterion to define the group of intermediate ties.

Although the k-means clustering provides reasonable outcomes, other clustering methods could be taken into account when classifying ties by their strength. In this study, the k-means clustering was considered appropriate, as it grouped all ties into three groups. Nevertheless, the k-means clustering in particular might not provide the best solution, as it is a heuristic method which aims to identify a solution that is reasonably close to the best possible answer. Although k-means clustering is also recommended for larger data-sets, one might consider the use of other clustering methods such as hierarchical clustering. Moreover, another clustering approach using 
the k-means clustering could include the criterion 'reciprocity' as a second variable besides the total tie strength values.

However, due to a lack of literature defining intermediate tie strength, particularly for the inter-organisational knowledge transfer context, the definition and identification of intermediate ties in the inter-organisational context is one of the most important key results of this study.

Further, the important roles of knowledge brokers (Delphi, 1997; Pawlowski \& Robey, 2004; Ruggles, 1998), and key sinks as well as key sources (Brogan \& Armstrong, 2005; Hanneman, 2005) have been identified in a number of publications. Nevertheless, in regard to managerial implications, the information on the key players in this study's networks, including knowledge brokers, key sinks and sources, may play a key role for future business decisions, such as new projects, alliances or learning initiatives among R\&D organisations. Knowledge brokers, such as CRIs, local governments or private businesses, could lead these future decisions. All three case conclusions have already outlined this importance according to the relevant literature (e.g. Cross et al., 2006; Liebowitz, 2005).

One of the key goals of this study was to provide important background information to increase collaboration among NZ R\&D organisations. Besides CRIs, local government and private businesses had very influential positions in the three networks. In all three cases, however, a CRI was the only provider of professional workshops to the public. Managerial implications may include a rethinking of the organisation of these workshops in regard to the inclusion of additional trainers from other organisations.

Besides CRI employees, trainers giving workshops in the hydrology domain could also include people from local government and private businesses. Trainers from local government would provide additional knowledge, as they work at the intersection of government, research and the application of these research outcomes and policies developed at central government departments. Private businesses seem to 
be in a similar position, but might be less closely connected to central government than local government. However, the application and commercialisation of knowledge may be even more important for private businesses. Their diverse perspectives and application examples would provide additional value-adding perspectives to all workshops participants.

To additionally increase collaboration, key organisations could collaborate in order to provide joint seminars online to an international audience. As environmental research in $\mathrm{NZ}$ is often world-leading, these joint seminars might provide an effective way to increase collaboration (seminar preparation) and to commercialise significant research knowledge internationally. Moreover, in the long-term, this may help build collaborative capabilities and push New Zealand's reputation as a world-leading research nation. The use of appropriate ICT to provide these learning services appears crucial.

Another managerial implication may be related to the use of ICT to support knowledge transfer. Besides using the email newsletter option of a related professional society, employees of the key participating organisations did not have any opportunity to connect via a common online platform to share their knowledge and experiences (apart from email). From an even more important perspective, this study revealed that research knowledge that is not part of the final product is often not saved nor provided to other people electronically. The introduction of a database and managerial efforts to support saving these data and knowledge could have enormous advantages for future projects, as it might help reuse knowledge and thus avoid reinventing the wheel.

Such an online platform at the inter-organisational level could be used for online collaboration and also additional online learning options that could be provided nationally or internationally. Especially in the international context, online learning might be a cost-effective option due to reducing travel costs. In the case of distance education costs could be reduced with using online learning material instead of 
sending study material by post. Moreover, online collaboration might be a good way to maintain new relationships that were initiated during face-to-face workshops and seminars. For example, an online platform with social networking features allows for easy maintenance of relationships. In the long-term, these relationships may lead to additional workshops or other important project opportunities. In addition, in the context of social networks, additional online collaboration would create latent ties (possible new relationships) and facilitate the creation of weak ties (Haythornthwaite, 2005). People can search for other people's profiles and contact them easily. The creation and long-term maintenance of weak ties may again provide future project opportunities.

The opportunity of online collaboration in addition to face-to-face workshops and seminars would provide a blended learning environment that supports individual learning needs. It would provide more flexibility in workplace learning and a better accessibility of knowledge online. This would particularly be of advantage to international and also locally remote organisations in New Zealand, such as several local government departments.

In addition, if some material could be provided in the form of online learning, costs to offer seminars to a lower number of participants would go down in the long-term. In the past, a high number of face-to-face workshops and seminars needed to be cancelled due a low number of participants (and thus high costs).

Regarding the current media used for advice giving and seeking activities in this study, email was clearly the favoured medium. Telephone calls and face-to-face conversation followed. However, the use of social software, which would be ideal to transfer informal and tacit knowledge (Avram, 2006) as well as meta-knowledge did not play an important role in the R\&D networks. An explanation could be the generation gap. According to Kupperschmidt (2001), social software is the type of communication medium used more often by the digitally-savvy generation born between 1980 and 1999. This would include few young people working in the R\&D sector at the time of this study. In addition, this could explain the preferred use of 
social software for inexperienced workers. Nevertheless, the younger generation will dominate the workforce in the future, and the use of social software will likely be a common medium to maintain contacts and transfer informal and tacit knowledge. Thus, regional proximity among key organisations may not play such an important role for the transfer of tacit knowledge, as it plays nowadays.

Unexpectedly, social networking tools, such as Facebook ${ }^{17}$, which could support the transfer of informal and tacit knowledge or contact details (meta-knowledge), were not accessible in certain groups of organisations, particularly in the public and private/public types. As social network sites fell in the category of sites with low information security ${ }^{18}$, some organisations, particularly government agencies in New Zealand, appeared to limit access to these social networking sites with the help of firewalls and filtering software. Therefore, groups of organisations such as CRIs and local governments were dependent on the given information security guidelines. Staff and students in universities, however, were actively involved in contacting others through Facebook.

Although remarkable improvements in terms of information security have been made since the earlier days of social networking sites ${ }^{19}$, these issues evoked a more negative than positive picture in regard to the use of social software in New Zealand organisations. This may have also affected the low use of social software, which is often integrated in social network tools, in this study. Similar considerations about organisational barriers to access these tools are stated in Chawner (2008).

In regard to this study's R\&D context, the unreliability of data and knowledge within social software, such as wikis and blogs, was also a concern for study participants. For example, one participant in Case 1 mentioned in the follow-up questions to the survey:

\footnotetext{
${ }^{17} \mathrm{http}: / /$ www.facebook.com

${ }^{18} \mathrm{http}: / /$ computerworld.co.nz/news.nsf/mgmt/680F55FBD8FED5DFCC2573F60083BC84

${ }^{19} \mathrm{http}: / / \mathrm{www}$. securecomputing.net.au/News/109053,facebook-defends-social-networkingsecurity.aspx
} 
"Because the quality is variable and is not edited, I don't spend much time looking at blogs "(Participant 1).

In an organisational context, it would therefore be necessary to moderate and edit communications in these tools in order to verify information and provide more reliable and value added knowledge to researchers.

The cross-case analysis further showed that study participants, particularly in Cases 2 and 3, associated their preferred knowledge service, online interaction with experts, with informing and participating characteristics. However, in Wenger and Ferguson's (2006) knowledge ecology model, online interaction with experts has an exclusive guiding characteristic. Therefore, it might be necessary to rethink the assignment of characteristics to knowledge services as currently available in the four sections of the model.

Finally, this cross-case analysis revealed the study participants' most preferred media characteristics were ease of use, quick transfer of knowledge and reuse of knowledge. Participants were asked to select the two most important ones for giving and seeking advice activities. However, in the follow-up questions to the survey, study participants could not always confirm their earlier selection. For example, one participant said:

"I don't agree ...... In my experience, having secure data, that is unambiguous and is not reproduced in different versions is as important as the ability to use it, reuse it or transfer it quickly. Also, security of data is an issue for study participants in the hydroelectric industry, and ...... has many of the study participants as clients" (Study participant 1).

In relation to Media Synchronicity Theory (MST), only two of the three most preferred characteristics could be considered: quick transfer of knowledge (transmission velocity) and reuse of knowledge (reprocessability). Nevertheless, (perceived) ease of use appeared to be an important characteristic too and might 
represent a combination of several other characteristics (e.g. quick transfer and reuse of knowledge). In regard to this consideration, the addition of this media characteristic to the set of media capabilities currently considered in MST would be suggested. In addition, and particularly important to group communication in the inter-organisational knowledge transfer context, would be another media capability that is currently not considered in MST: the capability of providing a trustworthy environment which includes secure transfer of data. It could be argued that the absence of the two factors - '(perceived) ease of use' and 'trustworthiness of the channel' hinders group communication from being focused towards the content of the communication, as people might, for example, put more effort into the physical transfer of knowledge (if the media is not easy to use). In addition, without having a trustworthy communication channel, people might communicate in a cautious way by omitting important details.

Implications for ICT support based on this study's key findings are described in the following chapter. 


\section{Implications for ICT support}

This chapter outlines practical implications for ICT to facilitate inter-organisational knowledge transfer in a $R \& D$ setting. These implications represent the major practical contributions of this study. They are based on key results of the cross-case analysis and the literature reviewed in Chapter 2, particularly in relation to the theoretical concepts of Media Synchronicity Theory (Dennis et al., 2008) and Wenger and Ferguson's (2006) knowledge ecology model. These implications helped answer this study's third sub-question: How can ICT support these knowledge transfer structures and processes?

The overarching goal was to provide ICT recommendations as an enhancement to current face-to-face workshops and seminars in the inter-organisational R\&D environment. In particular, these recommendations should support advice giving and seeking activities in the workplace. As an enhancement to current workshops, this support might be any form of collaborative formal or informal learning service, such as interaction with experts in a chatroom or discussion forum. Another way to build long-term relationships for advice giving and seeking activities between trainers and workshop participants might be to set up an inter-organisational e-mentoring programme that uses collaborative tools for knowledge exchange. In addition, the ICT recommendations might help organisations to enhance their learning services towards a blended learning approach combining ICT and non-ICT based services.

To develop these recommendations, a multiple-level perspective of possible ICT support was employed. This perspective classifies possible ICT by level of application and considers the individual, group and inter-organisational levels: 
- The individual level refers to ICT applications that support individuals in their personal knowledge work which may be embedded in inter-organisational knowledge transfer and learning.

- The group level relates to ICT support for advice giving and seeking activities in inter-organisational groups (e.g. workshop groups or project teams). These groups can be initiated by the firms themselves or could also be represented by professional societies in the respective areas of interest.

- The inter-organisational level relates to ICT systems able to connect organisations. This connection then allows members from diverse organisations to use the ICT provided at the group and individual levels.

The following key study results guided the development of possible ICT support:

Table 65: Key study results that guided implications for ICT

- Study participants' preferred media characteristics

- Study participants' preferred ICT-based knowledge services for learning

- The types of knowledge transferred in the three social networks

- Small-world network structure in all three cases

In line with the data collected, this thesis' key findings on study participants preferred media characteristics, the types of knowledge transferred and the structural network properties, such as the small-world network topologies, are all related to advice giving and seeking activities in the workplace. In addition, study participants' preferred ICT-based knowledge services for learning may help address the enhancement of current face-to-face workshops. Based on these results, the individual and group level ICT support should be collaborative in nature to support advice giving and seeking activities. Moreover, the key findings regarding the network structures (small-world networks) helped address the inter-organisational infrastructure and guided implications at the inter-organisational level. 


\subsection{Implications at the individual level}

In regard to the individual level, three different perspectives were considered to identify appropriate ICT support. Firstly, Wenger and Ferguson's (2006) knowledge ecology model and key results in regard to the study participants' most preferred knowledge services were considered, and possible ICT support was suggested. Secondly, the most preferred media characteristics for advice giving and seeking activities in regard to MST were taken into account for further ICT recommendations; and thirdly, the direction of knowledge transfer for knowledge givers and seekers was considered.

Firstly, Wenger and Ferguson's (2006) model of guiding, informing, exploring and participating knowledge services allowed for recommendations for ICT support. These services, which represent a functional perspective, are briefly described below in relation to Wenger and Ferguson's (2006) model.

Guiding: With guiding knowledge services, experts guide people with the aim to apply both knowledge and skills successfully to new situations. The guiding service can be compared to more practical hands-on workshops. Additional guiding knowledge services can be mentoring and coaching, web conferencing and interaction with experts.

Informing: With informing knowledge services, experts inform people about common theories and practice in a neutral environment. These services are comparable to more lecture-based workshops and seminars. Additional informing services can be blended learning, continuous learning or learning management.

Participating: People participate in a community to create understanding. The participating service can be again compared to practical hands-on workshops. Additional participating services can be online communities of practice, discussion forums and chat or instant messaging. 
Exploring: For learning, people explore resources for ideas and practical insights in a neutral environment (Wenger \& Ferguson, 2006, p.88). These services may be comparable to a library visit or searching the Internet or databases for articles etc. Exploring services in an organisational setting can be content repositories, learning portals or e-books.

Table 66 suggests possible ICT-based knowledge services for each service characteristic.

Table 66: Mapping of knowledge service characteristics and ICT support (adapted from Wenger \& Ferguson, 2006)

\begin{tabular}{|l|l|l|}
\hline Characteristic & Preferred knowledge services & Other possible knowledge services \\
\hline Guiding & Online interaction with experts & $\begin{array}{l}\text { E-mentoring or remote coaching, web } \\
\text { conferencing, online labs }\end{array}$ \\
\hline Informing & Online learning & Blended learning, virtual classrooms \\
\hline Participating & $\begin{array}{l}\text { Online discussion forums and } \\
\text { chat }\end{array}$ & $\begin{array}{l}\text { Online communities of practice, instant } \\
\text { messaging }\end{array}$ \\
\hline Exploring & E-books & Learning portals \\
\hline
\end{tabular}

These considerations of preferred and appropriate knowledge services in this study's context suggest the following proposition:

Proposition 1: "For inter-organisational knowledge transfer in a small-world network, guiding knowledge services, such as online interaction with experts, may be supported by e-mentoring, web conferencing or online labs".

Secondly, Media Synchronicity Theory (MST) was employed to identify appropriate ICT at the individual level. Media should fit the users' needs; otherwise they will not be appropriated and used. Therefore, participants in this study were asked for their preferred media characteristics for advice giving and seeking activities. Study participants were consistently interested in the following two options: 
Table 67: Most preferred media characteristics

\begin{tabular}{|l|l|}
\hline Most preferred media characteristics & Media \\
\hline $\begin{array}{l}\text { The ability to save information for later reuse } \\
\text { (reprocessability) }\end{array}$ & $\begin{array}{l}\text { Synchronous and asynchronous } \\
\text { electronic conferencing, email }\end{array}$ \\
\hline $\begin{array}{l}\text { Quick transfer of information (transmission } \\
\text { velocity) }\end{array}$ & $\begin{array}{l}\text { Video-conference, synchronous instant } \\
\text { messaging, synchronous electronic } \\
\text { conferencing }\end{array}$ \\
\hline
\end{tabular}

These two options, as illustrated in Table 67, suggest a mix of media having either low or high synchronicity to support the communication processes of conveyance (transmission) and convergence (shared understanding). For example, a mix of email (supporting reprocessability) and instant messaging (supporting transmission velocity) would be the appropriate ICT support for this context.

The ability to save information for later reuse (reprocessability) shows high synchronicity in relation to asynchronous media. This type of media allows the user to save and reuse knowledge at a later stage. In contrast, the transmission velocity shows high synchronicity using synchronous media. This type allows the user to give or receive knowledge quickly.

Although synchronous media tend to support higher synchronicity in communication, a shared understanding (high synchronicity) does not need to be achieved (Dennis et al., 2008). Nevertheless, in the context of effective knowledge transfer, a shared understanding and therefore high level of convergence (Dennis et al., 2008) appear crucial.

Therefore, a mix of synchronous and asynchronous media may be best suited to support both preferred media characteristics. This mix of media, supporting communication performance in different ways according to the preferred media characteristics for specific purposes, confirms Dennis et al.'s (2008) suggestion that the "best medium for a given situation may be a combination of media" (Shahriza et al., 2005; Watson-Manheim \& Belanger, 2007 cited in Dennis et al., 2008, p.588). In 
the context of social networks, a mix of multiple media (media multiplexity) is more likely to be used by people who are in strong social relationships in a network (Haythornthwaite, 2005) $^{20}$. Thus, in the context of this study, a mix of these diverse media might be best adopted by more strongly tied groups of organisations, such as CRIs, local government agencies and private businesses.

In line with these considerations, the following two propositions are suggested:

Proposition 2: "In regard to reprocessability, knowledge transfer in an interorganisational small-world network can be supported by using media with low synchronicity such as email or asynchronous conferencing”.

Proposition 3: "In regard to high transmission velocity, knowledge transfer in an inter-organisational small-world network can be supported using media with high synchronicity such as video-conferencing or instant messaging”.

Thirdly, regarding the direction of knowledge transfer at the individual level, push and pull technologies may support givers and seekers of knowledge. Interview data indicated study participants' strong interest in receiving relevant information automatically. In terms of ICT support, seekers could take advantage of pull technologies, such as RSS or automatic email notification systems. On the other hand, givers of knowledge may want to distribute knowledge to a larger group of people. This would be possible using distribution services such as email lists. In contrast to this more traditional distribution service, a more up-to-date service allows for the mass distribution of text messages (SMS: short message service), for example in the form of micro-blogging (e.g. twitter ${ }^{21}$ ). With regard to these considerations, the following proposition is suggested:

\footnotetext{
${ }^{20}$ Section 2.4.3.1.

${ }^{21} \mathrm{http}: / /$ twitter.com/
} 
Proposition 4: "For giving knowledge to, and seeking knowledge from, peers in an inter-organisational small-world network, ICT based on push and pull technologies are appropriate".

\subsection{Implications at the group level}

In this study's context, the group level refers to inter-organisational workshop/seminar groups which could be supported by additional ICT-based learning services such as online interaction with experts, which was the most preferred knowledge service in the study's three social networks.

At the group level, the key results about the types of knowledge transferred for advice giving and seeking activities in the social networks were important. In particular electronically unavailable knowledge played a key role for ICT support. In addition, for all three networks, tacit informal factual knowledge, such as unpublished research material, appeared to be an important type of knowledge. In addition, tacit metaknowledge and tacit informal how-to knowledge were also important in two of the three networks.

\section{Social software and social network tools}

Givers and seekers of advice who were interested in transferring informal factual and how-to knowledge indicated that this knowledge was not available electronically. In order to make it accessible, social software, such as wikis and blogs, could be utilised to transfer, save and organise informal knowledge that is often communicated in a semi-structured way.

Moreover, in regard to social networking, an online social network tool (such as $\mathrm{NING}^{22}$ ) would provide an opportunity for making experts' contact details visible to others online. NING, in particular, allows organisations to create their own social

${ }^{22}$ http://www.ning.com/ 
network for a particular topic. This can include specific membership databases. Profile pages in such a social networking tool represent a kind of yellow pages directory for locating experts. Yellow pages are typically part of organisational KM initiatives (Stankosky, 2005). To support advice giving and seeking activities in the workplace after face-to-face workshops, an inter-organisational, but otherwise private, social network could be set up for continuing education and long-term relationships.

In addition, tacit meta-knowledge, in the form of references to other sources of knowledge, could be made electronically available and shared by a social bookmarking system that allows for collecting and sharing interesting online references.

\section{Peer-to-peer applications}

In addition to social software and social network tools, peer-to-peer applications are considered in particular for ICT support at the group level. Some social software or social networking tools may include this type of application.

As each social network in this study, and therefore each group of people, represented a decentralised network structure, peer-to-peer applications that connect people directly appeared most useful as a recommendation at the group level in this interorganisational environment. Peer-to-peer applications to support for example online interaction with experts could be Instant Messaging tools (Microsoft Messenger, Skype) or live online chatrooms.

In regard to organisational interests such as keeping costs low, these applications could ideally be based on Open Source e-learning software that includes these collaboration tools and social networking features (such as Moodle ${ }^{23}$ ). A brief description of Moodle has been given in Section 3.8 in Chapter 3. Such e-learning software could also effectively complement existing face-to-face workshops.

\footnotetext{
${ }^{23} \mathrm{http}: / /$ www.moodle.de/
} 
Another rather cost-effective solution could be taken up by renting software services from specialised providers. These software rental services are referred to as cloud computing nowadays, but these application software providers (ASPs) have been around for several years. In this case, the software and hardware is located with and maintained by the provider and users pay rental fees.

These considerations on social software, social networking tools and peer-to-peer applications lead to the following proposition:

Proposition 5: "The transfer of electronically unavailable and tacit knowledge (informal and meta-knowledge) in a small-world network can be supported by social software and social network tools that include peer-to-peer applications".

The next section on ICT support at the inter-organisational level follows up on the considerations at the individual and group levels.

\subsection{Implications at the inter-organisational level}

In contrast to the individual and group levels that focussed on collaborative services for advice giving and seeking activities in the workplace, the inter-organisational level aims to take the overarching infrastructure into account. At the inter-

organisational level two important aspects were considered in order to develop propositions for ICT support. Firstly, the identification of this study's networks as small-world networks played a key role. Secondly, secure communication in an interorganisational context was considered.

This study identified three small-world networks with a decentralised structure. In this regard, decentralised ICT and particularly a decentralised KM system may be an appropriate support solution. A knowledge management system can be defined as "an 
ICT system in the sense of an application system or an ICT platform that combines and integrates functions for the contextualised handling of both explicit and tacit knowledge throughout the organisation or that part of the organisation that is targeted by a KM initiative" (Maier, 2001, p.76). Examples of KMS could be intranet infrastructures, document and content management systems, artificial intelligence technologies, business intelligence tools, visualisation tools, groupware or e-learning systems (Maier \& Hädrich, 2006).

A decentralised KMS, in particular, connects computers and people directly (rather than a central KMS which has central control). Decentralised systems aim to provide efficient solutions to maintain a knowledge repository (Maier \& Hädrich, 2006; Schmitz et al., 2006). An example of a decentralised KM solution which is based on a peer-to-peer architecture is Groove ${ }^{24}$. Groove, in its current version (2007), is distributed by Microsoft. In its basic features, it supports collaborative document development and management integrated in business processes.

Such a decentralised ICT solution may be especially suitable for inter-organisational settings, as knowledge processes often cross organisational boundaries (Maier \& Hädrich, 2006). Nevertheless, decentralised KM systems still lack technical and organisational maturity, especially in the case of inter-organisational knowledge transfer (Maier \& Hädrich, 2006).

In line with this study's identified small-world network topologies, a decentralised peer-to-peer knowledge management system (KMS) thus seems appropriate. A decentralised system allows several powerful groups of organisation to use peer-topeer connections efficiently. Moreover, peer-to-peer systems typically reduce the barriers of knowledge transfer at the individual level (Maier \& Hädrich, 2006). Individual knowledge workers are motivated to participate in such a KMS and share the benefits (Maier \& Hädrich, 2006). In the context of this study, a decentralised

${ }^{24} \mathrm{http} / / /$ office.microsoft.com/de-de/groove/default.aspx 
KMS would facilitate advice giving and seeking activities in the workplace. These considerations suggest the following proposition:

Proposition 6: "Inter-organisational knowledge transfer in small-world networks may be supported by peer-to-peer KMS".

Inter-organisational knowledge transfer requires secure communication, as partners in one project are often also competitors in other projects. Therefore, Virtual Private Network services would be best suited to support such a scenario. A VPN uses a public network (such as the Internet) for private (e.g. organisational) communication purposes (Lipp, 2006).

An Extranet-VPN, in particular, allows external organisations controlled access to an organisation's intranet (Lipp, 2006). An Extranet-VPN opens a private intranet up to external users who can then access certain resources within this intranet. A reliable firewall and authentication mechanism play a key role in this regard for interorganisational knowledge transfer.

For example, an Extranet-VPN infrastructure may open up one organisation's intranet to external users in regard to collaborative use of a portal solution for knowledge transfer (Villian et al., 2004; Zheng et al., 2004). Particularly in the interorganisational context, portal solutions allow for sharing knowledge among a group of diverse users and organisations (e.g. van Baalen et al., 2005; Zanner, 2006). According to van Baalen et al. (2005), a portal for inter-organisational knowledge transfer especially supports the exchange of explicit knowledge.

However, in the context of this study, it became apparent that public organisations, such as CRIs and government departments, had to follow strict IT security guidelines and would therefore not be able to open up an intranet to external users. Thus, an inter-organisational portal solution might be best hosted externally. This would also support earlier arguments to use services of cloud computing or ASPs. 
These considerations suggest the following proposition:

Proposition 7: "Knowledge transfer in social networks, at the inter-organisational level, may be supported by a portal solution that is hosted externally".

\subsection{Summary}

This section about the practical implications considered ICT support at three levels the individual level, the group level and the inter-organisational level. At the individual level, three implications for ICT were developed. Firstly, study participants' most preferred knowledge service was online interaction with experts, which could be supported by collaborative services such as e-mentoring. Secondly, individuals' most preferred media characteristics for advice giving and seeking activities - quick transfer and reprocessability of knowledge - would suggest a mix of synchronous as well as asynchronous collaboration tools to best support both characteristics. Finally, knowledge givers and seekers would be best supported by using push and pull technologies to facilitate their personal knowledge transfer needs. At the group level, the type of knowledge most frequently transferred when giving advice to or seeking advice from peers at the workplace - tacit knowledge - has led to the recommendation of ICT support in the form of social software and social network tools which include peer-to-peer applications. These ICT recommendations

given for the individual and group level are collaborative in nature and thus may facilitate the creation of new ties in a social network (Haythornthwaite, 2005).

At the inter-organisational level, peer-to-peer KMS have been suggested for knowledge transfer in small-world networks. In addition, portals for interorganisational knowledge transfer, which are based on an Extranet-VPN infrastructure, are recommended for the study's context. 
In summary, the combination of this study's key results and the theoretical concepts employed - MST and the knowledge ecology model - allowed the researcher to develop a number of recommendations in the form of propositions (Chapter 8). The overarching goal in this regard was to suggest ICT support for inter-organisational knowledge transfer (advice giving and seeking activities) in addition to the current face-to-face workshops. This could help develop an effective inter-organisational blended learning environment supporting diverse study participants' needs. Table 68 summarises the possible ICT support at each level in relation to the key study results. 
Table 68: Summary: Levels of ICT support

\begin{tabular}{|c|c|c|}
\hline $\begin{array}{l}\text { Level of } \\
\text { application }\end{array}$ & Key results & ICT support \\
\hline $\begin{array}{l}\text { Individual } \\
\text { level }\end{array}$ & $\begin{array}{l}\text { - The most preferred knowledge } \\
\text { service (Wenger \& Ferguson, 2006) } \\
\text { of study participants was online } \\
\text { interaction with experts; } \\
\text { - The most preferred media } \\
\text { characteristics in relation to MST } \\
\text { were reprocessability and } \\
\text { transmission velocity; } \\
\text { Diverse levels of interaction } \\
\text { between network members included } \\
\text { the direction of knowledge transfer } \\
\text { (knowledge giving and seeking) }\end{array}$ & $\begin{array}{l}\text { - A mix of } \\
\text { synchronous and } \\
\text { asynchronous } \\
\text { media; } \\
\text { Social software } \\
\text { and social network } \\
\text { tools (access to } \\
\text { expert profiles and } \\
\text { contact details); } \\
\text { Push and pull } \\
\text { knowledge } \\
\text { services; } \\
\text { Open Source e- } \\
\text { learning software } \\
\text { that offers peer-to- } \\
\text { peer collaboration }\end{array}$ \\
\hline Group level & $\begin{array}{l}\text { - The types of knowledge transferred } \\
\text { most frequently were tacit informal } \\
\text { and meta-knowledge; in addition, } \\
\text { electronically unavailable } \\
\text { knowledge was important; } \\
\text { The most preferred ICT-based } \\
\text { knowledge service in regard to } \\
\text { additional learning was online } \\
\text { interaction with experts }\end{array}$ & $\begin{array}{ll}\text { - } & \text { Social software } \\
\text { - } & \text { Social network } \\
\text { tools } \\
\text { - } \\
\text { Peer-to-peer } \\
\text { applications }\end{array}$ \\
\hline $\begin{array}{l}\text { Inter- } \\
\text { organisational } \\
\text { level }\end{array}$ & $\begin{array}{l}\text { - The identified network topology in } \\
\text { all three cases was a small-world } \\
\text { network structure that included } \\
\text { several key players per network and } \\
\text { a high number of intermediate ties; } \\
\text { - Secure knowledge transfer was } \\
\text { important to study participants }\end{array}$ & $\begin{array}{l}\text { Decentralised } \\
\text { (peer-to-peer) } \\
\text { knowledge } \\
\text { management } \\
\text { system (KMS); } \\
\text { Portal solution that } \\
\text { is externally } \\
\text { hosted }\end{array}$ \\
\hline
\end{tabular}

The next chapter outlines conclusions and implications of this study. 


\section{Conclusion and Implications}

This chapter summarises this research and identifies its major contributions and their implications. An overview of the research presents a summary of the context, the gaps in the literature, the research questions, research design and the findings of this thesis. The major theoretical and practical contributions are outlined. In addition, implications for future research are drawn. Finally, study limitations are outlined and a conclusion is given.

\subsection{Overview of the research}

The purpose of this study was to investigate how knowledge is transferred and how this transfer could be facilitated by ICT to support collaboration among R\&D organisations in inter-organisational social networks.

\section{The context}

The New Zealand government has decreased R\&D funds during the last decade, especially for CRIs. Therefore, key players in R\&D, such as CRIs, need to work smarter with available funds and also increase business funding in order to compensate for the decrease in government funding. This could be achieved by focussing on commercialising crucial research knowledge and cooperating better in formal and informal networks. An improvement in knowledge transfer among organisations and its support by appropriate ICT was therefore seen as playing a key role in helping to achieve this goal. In addition, well-supported knowledge transfer could help reuse knowledge and drive innovation.

In order to improve knowledge transfer, this thesis aimed to investigate important structural aspects, such as direction of knowledge transfer and tie strength, related to knowledge transfer in social networks. Furthermore, the supportive role of ICT for knowledge transfer was explored. In this regard, the study identified suitable ICT to support knowledge transfer and learning in an R\&D network at the individual, group and inter-organisational levels. 


\section{Related literature and research questions}

Knowledge-intensive R\&D activities have seldom been investigated from a social network perspective. As Allen et al. (2007) point out, there is a lack of research addressing the issue of knowledge transfer in inter-organisational social networks, specifically in the R\&D sector. By employing social network analysis (SNA), this study addressed this gap and analysed and identified inter-organisational knowledge transfer structures and processes among organisations by focussing on three cases. The concepts underlying social network analysis played a significant role in informing this research that was guided by the following main research question:

What are the knowledge transfer structures and processes among organisations within an R\&D network?

In particular, this study investigated advice networks, as a considerable number of previous studies used advice giving and seeking activities as the key knowledge transfer processes when looking at how people transfer knowledge in their professions (e.g. Cross et al., 2001; Haythornthwaite, 1996; Krackhardt \& Hanson, 1993). These advice giving and seeking activities appeared well suited to reflect informal knowledge transfer processes in the workplace.

The first two research sub-questions were:

1) What are the key structural properties of advice networks and their implications for knowledge transfer?

2) Which types of knowledge are transferred in the advice networks?

Answering these provided a background, together with the identification of study participants' preferred media characteristics and ICT-based knowledge services for learning, to identify how ICT can facilitate this knowledge transfer. 
Implications for knowledge transfer and ICT support could be drawn, particularly for knowledge transfer in an inter-organisational learning environment. Possible ICT support was categorised into three levels that appeared useful in this study's context: the individual, the group level and the inter-organisational level. For this purpose, theoretical key concepts of Media Synchronicity Theory (Dennis et al., 2008) and Wenger and Ferguson's (2006) knowledge ecology model informed this research. This helped answer the third sub-question of this research:

3. How can ICT support these knowledge transfer structures and processes?

\section{The research design}

This thesis employed a mixed methodology approach with case research as the leading research strategy. SNA methods and techniques were embedded in this strategy as a complementary methodology to improve understanding of the phenomenon of interest: knowledge transfer between organisations in the New Zealand R\&D sector. Case research was conducted with a multiple case design, comprising three inter-organisational social networks in the NZ environmental sector. In combination with a mixed methodology approach, the research was based on an integrated approach of interpretivist and positivist philosophical stances.

The three case studies were conducted in sequence. A sequential approach was also employed for data collection and analysis within each case. This started with a qualitative phase that helped to understand the R\&D network environment including co-operation, competition and collaboration among organisations. In addition, this phase helped gather data about social networks in the R\&D network in order to develop an instrument for the second part of the sequential data collection and analysis approach. In this second phase, SNA was used to collect data about social relationships and their knowledge transfer, including quantitative data of the contact frequency in relationships. A third, and final, phase of data collection, again 
qualitative in nature, posed follow-up questions about survey results to study participants with the aim of explaining and confirming key results.

\section{The research findings}

This thesis identified various aspects regarding structural properties that may influence knowledge transfer in inter-organisational social networks:

\section{- The key players}

Key sinks, key sources and (key) knowledge brokers were identified in the three networks. With regard to the analysis of the levels of interaction and centrality measures, all three cases had three key players in common: CRIs, local government and private businesses.

\section{- The network structures}

All three social networks could be identified as small-world networks. This decentralised structure shows some degree of local clustering represented by a relatively high clustering coefficient and a large number of short paths in the networks.

\section{- The importance of intermediate ties}

The definition of intermediate ties was crucial. In particular, the k-means clustering in combination with a subsequent investigation of the levels of reciprocity was suggested for defining intermediate ties. The k-means clustering initially grouped all total tie strength values in a network into three groups: weak, intermediate and strong ties. Then, an investigation of the levels of reciprocity at the borderlines of this initially identified group was carried out.

The significant roles of intermediate ties in social networks have also been highlighted. Besides their numerous appearances in this study's social networks, the intermediate ties have represented two specific roles. Their significance was represented by either Role 1, which entailed linking otherwise weakly linked 
groups of organisation to the network, or Role 2, linking two otherwise very strongly connected groups of organisation (key players). Thus intermediate ties might help receive new knowledge from outside the network with the help of Role 1 ties and also help access the local networks of other strongly connected network members with the help of Role 2 ties.

\section{- The types of knowledge transferred}

The most important electronically available types of knowledge, throughout all cases, were formal factual knowledge and references to other sources of knowledge (meta-knowledge). Formal factual knowledge could represent published research material, whereas other sources of knowledge represent metaknowledge and could be electronically available in the form of literature references in a database. The most important electronically unavailable type of knowledge was non-codified informal factual knowledge. This type could represent additional unpublished research material that has not been written down.

\section{- The appropriate centrality measures for these knowledge networks}

In regard to these types of transferred knowledge, Information Centrality was chosen as the most appropriate measure, besides closeness and flow betweenness. Attention was paid to literature recommending considerations about the type of knowledge transferred and the way in which this knowledge is typically transferred in the network as suggested by Borgatti (2005).

\section{- The media use}

The predominant medium used in all three networks was email. This was followed by other traditional media, such as face-to-face meetings and phone calls. Relatively new ICT-based media such as discussion forums, videoconferencing and social software ranked third and were rarely used. 


\section{- Study participants' preferred media characteristics}

Study participants' preferred media characteristics were quick transfer of knowledge (transmission velocity), ease of use and the ability to save information for later reuse (reprocessability). Two of these three characteristics - transmission velocity and reprocessability - are also employed in Media Synchronicity Theory (Dennis et al., 2008).

\section{- ICT-based knowledge services for learning}

Finally, the most preferred ICT-based knowledge service for learning at the workplace in all three networks was 'online interaction with experts'. In this regard, study participants were not only interested in guiding and informing knowledge services for learning, but also in participatory ICT-based learning initiatives. Wenger and Ferguson's (2006) knowledge ecology model informed these results on knowledge services and their characteristics.

Together with the key results on the structural properties in the social networks, study participants' preferred media characteristics and ICT-based knowledge services for learning helped develop implications for ICT support.

The following section outlines the quality assurance undertaken for this thesis.

\subsection{Quality assurance}

This section outlines the quality aspects considered for this mixed methods research including case research and social network analysis.

Mixed methods research considers both quantitative and qualitative quality concepts. Quantitative research traditionally employs the concepts of reliability and validity to ensure quality. Validity needs to be assured for the constructs and content used. Reliability refers to the consistency of the results. Qualitative research typically 
applies the concept of trustworthiness as the fundamental of quality and rigour to which validity and reliability have traditionally referred (Seale, 1999). Due to having a mainly qualitative approach, this case research study applied the concept of trustworthiness, which is described first. Then, for the quantitative part of the embedded SNA approach, specific SNA issues are addressed.

\section{Quality aspects in case research}

Lincoln and Guba (1985) recommend four criteria for addressing trustworthiness: credibility, which addresses internal validity; transferability, which refers to external validity; dependability, which refers to reliability; and confirmability, which addresses objectivity issues. These four criteria are discussed in relation to concepts of validity and reliability as suggested by Yin (1993). In addition, efforts are described which were undertaken to enhance the validity of a variety of methods used in this study including participant observation, the case study method and interviews (Ridenour \& Newman, 2008).

Credibility is related to internal validity and refers to the richness of gathered data and the analytical abilities of the researcher (Patton, 1990). Credibility can be strengthened, for example through methodological triangulation in a case study (Ridenour \& Newman, 2008).

This study ensured credibility through multiple data collection techniques (participant observation, interviews, surveys and a Wiki tool) that allowed for some triangulation of data. For example, follow-up questions (using the Wiki tool) were posed to workshop participants to confirm survey results. Participant observation focussed on social interactions between workshop participants from different organisations. Although participants were aware of the researcher's presence as an observer, interactions with participants were kept to a minimum in order to not disrupt the workshop. There was not much reactivity among participants to the presence of the 
observer. Therefore the validity could be enhanced as is suggested by Ridenour and Newman (2008).

In addition, a well structured process was used for both interviewing (interview guidelines), and recording and transcribing interviews (Lincoln \& Guba, 1985). Credibility was strengthened by the prior development of interview guidelines, and each case was studied in relation to the literature review carried out as a part of this study (Yin, 1993). A copy of the interview guidelines can be found in Appendix 40. Moreover, study participants could check their interview transcripts and feedback on key findings (business report) was provided which enhanced credibility (Lincoln \& Guba, 1985). The application process to meet Victoria University of Wellington's Code of Human Ethics also strengthened the credibility of this study.

Transferability is related to external validity and therefore the generalisation of research findings. The transferability of the findings of a case research project to another situation is dependent on the comparability between the study context and the context to which it is being transferred. The researcher is therefore required to provide sufficient information about the comparability of a specific context to another environment (Lincoln \& Guba, 1985).

In this study, the application of a multiple case study and the appropriate use of procedures for coding and analysis helped to enable such a comparison (Lincoln \& Guba, 1985; Miles \& Huberman, 1994). This multiple case study helped generate analytical generalisations through replication and by corroborating the results (Yin, 1993). Based on certain similarities among the three social networks studied, the transferability of findings from one social network to another was described in the cross-case analysis chapter.

Moreover, key results were strongly related to the analysis of social interactions that can be decoupled to some extent from the specific case research findings. For example, results of this study may be easily transferred to other knowledge networks 
in other countries and other contexts. This study also retrieved and documented suitable contextual information about each case in order to make the results transferable. These case descriptions can be found in Chapters 4-6.

The dependability of qualitative research, which is related to the reliability and consistency aspects, may be enhanced by reviewers auditing the research process and its findings for consistency (Lincoln \& Guba, 1985). According to Krefting (1991), dependability can also be improved by stepwise replication, triangulation and densely describing the research methods.

In this study, regular supervisory meetings and meetings with other PhD students ( $\mathrm{PhD}$ group) ensured the consistency of the research process and the findings. In addition, triangulation of sources, such as interviews, observation notes and other documents, was used. Moreover, the multiple case study provided the opportunity for a step-wise replication and cross-checking of interpretations, minimising misinterpretations and finding other issues that might need clarification.

Confirmability represents a quality standard that can be achieved by a high degree of explanation of how the research findings have been reached in order to overcome potential subjectivity problems. It is related to construct validity. According to Yin (1993), techniques such as gathering multiple sources of evidence, establishing a chain of evidence and allowing key participants to review the initial case study, may help to address these issues. Moreover, audit trails including raw data, analysis notes, reconstruction and synthesis products, process and personal notes and preliminary developmental information are recommended (Lincoln \& Guba, 1985).

This study used multiple sources of evidence that allowed for cross-checking of results to some extent. Further, Case 1, which had pilot study characteristics, helped examine the sequential data collection process. This helped identify and address problems and issues in order to enhance the logic of the research process for the following cases and therefore build a strong argument for construct validity (Yin, 
1993). In addition, feedback on key results (business report) was provided by study participants (Lincoln and Guba, 1985).

\section{Quality aspects in SNA}

This section outlines possible issues in SNA regarding anonymity of study participants, withdrawal of consent and reliability of the reported data. In addition, steps made to ensure the validity and reliability of the online survey, in particular, in regard to the quantitative SNA questions, are outlined.

There are ethical and other critical issues that need to be considered when carrying out a SNA study. According to Borgatti and Molina (2003), network studies that focus on mapping relationships between study participants are based on the identification of these participants. Thus, anonymity is impossible in the data collection phases. Nevertheless, the researcher can ensure confidentiality by the use of disguised names or untraceable identification numbers with regard to the analysis and reporting stages (Borgatti \& Molina, 2003).

This research studied social relations and inter-organisational knowledge transfer at the inter-organisational level. Therefore individual study participants could not be identified in any way. However, in the data collection some key organisations were not disguised, as participants were asked to indicate their social relationships to people working in these organisations. Nevertheless, in the analysis and results reports organisational names were either replaced by disguised names or aggregated to a higher level describing groups of organisations (e.g. CRIs, central government and private businesses).

Another difficulty may arise, if social network members decide to withdraw consent during the research project. This could raise issues for the researcher with regard to their inclusion or non-inclusion (Borgatti \& Molina, 2003). The option to withdraw was outlined in the information provided to potential study participants before the 
data collection. No study participants withdrew during this study. However, two study participants wanted to clarify permission to take part in the survey with their superior. In order to provide additional information, the survey was emailed to these potential participants in the form of an electronic document for a preview.

One substantive issue in SNA research is the actor's self-reporting during the data collection (Wasserman \& Faust, 1994), which may affect the reliability of the data used for analysing relational structures. This self-reporting contains potentially inaccurate data, because half of what is reported seems to be incorrect in some way. This study tried to eliminate this issue by using a three-phase approach for data collection. These three phases allowed the researcher to cross-check data from interviews, the survey and follow-up questions to some extent. Thus, this triangulation method helped strengthen the reliability of data.

The following sections describe the steps made to ensure reliability and validity of the online survey distributed in Phase 2.

\section{Reliability}

In regard to survey reliability for SNA, most research studies have investigated the in-practice performance of instruments (Carrington et al., 2005). Nevertheless, the reliability and validity of social network measurement instruments were evaluated by Ferligoj and Hlebec (1999). Their work includes recommendations on measurement techniques, particularly for listing network members, and different measurement scales used for evaluating tie strength. Ferligoj and Hlebec (1999) recommend using a full list of network members for data collection as this "simplifies the reporting task for respondents, and it increases the number of reported ties" (Ferligoj \& Hlebec, 1999, p.126).

Drawing on Ferligoj and Hlebec's (1999) recommendation, a full list of network members was considered to be the best option for this study. Some key (groups of) 
organisations were therefore elicited in data collection during Phase 1 before the online survey. These key (groups of) organisations were then employed as network members in the survey that collected SNA data. In Case 1, five key organisations were nominated. In Cases 2 and 3, six key organisations (or groups of key organisations in an aggregated form) were nominated.

\section{Validity}

\section{Content validity of survey items}

In terms of measurement scales used for evaluating tie strength, this study applied a fixed choice format. Tie strength was assessed by contact frequency using the following options: at least once a week, at least once every month, at least once every three months, at least once every six months or never. The concept of employing contact frequencies for measuring advice giving/seeking activities is a widely used concept in SNA studies (e.g. Cross \& Borgatti, 2001; Krackhardt \& Hanson, 1993; McEvily \& Zaheer, 1999). In addition, senior IS researchers at the School of Information Management (SIM) at Victoria University of Wellington were asked to provide their opinion on the content validity of the scales. Such a judgement round is

a typical method to validate items in a questionnaire (Davis, 1989; Moore et al., 1991). The senior researchers identified items that were worded unclearly and suggested other wordings, such as "at least once every month" or "at least once every 6 months", that have also been used in other studies. These wordings provided a clear separation among all items.

Study participants were asked to select one option (e.g. at least once every month) for each network member when considering contact frequency during the last year. This was considered the most user-friendly way for answering these questions. However, study participants were required to list a particular number of responses without the possibility of subjectively evaluating (rank order) these given alternative answers (Wasserman \& Faust, 1994). 


\section{Content validity of measures}

In terms of content validity of measures in SNA, there is some research on the extent of actual measurement compared to intended measurement results. For example, Petróczi et al. (2007) measured tie strength in virtual social networks and demonstrated that the triangulation of methods, especially interviews with study participants after the questionnaire, "provided evidence for validity" (p.47) of their measures. This study conducted a follow-up data collection phase (Phase 3 ) after the SNA survey in order to confirm and explain results in relation to knowledge transfer and media use in inter-organisational social relationships.

\section{Pre-test of the survey}

To ensure content and construct validity, a pre-test of the survey with fellow researchers and then the conduction of Case 1 with respondents from the target population helped identify issues associated with the survey design. After this pre-test (Case 1) lessons learned were integrated into the data collection design of the following cases (Cases 2 and 3). This included a structural redesign of the survey as well as several changes in phrasing as outlined in Section 3.7. However, the structure

of the questions about the contact frequencies, including the number and wording of items, remained the same in all three cases. Besides these questions, all other questions in the survey did not aim to measure any constructs.

The following section outlines the academic and practical contributions of this research. 


\subsection{Contributions of the research}

This chapter summarises the key research contributions of this study for academics and practitioners.

\subsubsection{Academic value of the research}

The following major topics identified as key contributions are:

- The identification of small-world network topologies in inter-organisational advice networks

- The identification and definition of intermediate ties

- A recommendation on the most suitable SNA centrality measures in knowledge networks (dependent on the type of knowledge transferred).

\section{Small-world network topologies in the R\&D sector}

The identification of all three social networks as small-world networks, due to their high clustering coefficients and high number of short paths (Kwon et al., 2007; Watts \& Strogatz, 1998) illuminates some important aspects for inter-organisational knowledge transfer. Study participants in these decentralised networks have the advantage of having relatively easy access to inter-organisational knowledge sources. In addition, a small-world network structure has been found to be the ideal structure to facilitate and optimise knowledge transfer and knowledge creation in interorganisational knowledge networks (Cowan, 2004; Cowan \& Jonard, 2004; Schilling \& Phelps, 2007)

Thus, these small-world network topologies provide a reasonably well structured opportunity for inter-organisational knowledge transfer, apart from inhibitors such as 
remote locations or competition issues. To overcome the disadvantage of a remote location, ICT support may be most useful for organisations. ICT support may also help elicit and store knowledge resulting from inter-organisational knowledge transfer. This seems particularly significant for tacit knowledge, as it is not easy to capture and transfer. Nevertheless, the appropriate storage of explicit knowledge can also significantly help to reuse existing knowledge and therefore prevent 'reinventing the wheel'.

In small-world networks, ICT can efficiently support information processing (Kwon et al., 2007). ICT allows for connections across different organisations, and these connections help encourage collaboration among network members. As small-world networks are fairly decentralised structures, a similarly decentralised ICT solution might be ideal to facilitate the knowledge transfer structures and processes. Implications for ICT to support the small-world networks were outlined in Chapter 8.

\section{The identification and definition of intermediate ties}

The identification and definition of intermediate ties might represent the most important key finding of this study. In particular, the definition of intermediate ties when considering more than one indicator for tie strength (e.g. in inter-organisational social networks) was crucial. In particular, the k-means clustering in combination with a subsequent investigation of the levels of reciprocity was suggested. The kmeans clustering initially grouped all total tie strength values in a network into three groups: weak, intermediate and strong ties. Then, an investigation of the levels of reciprocity at the borderlines of this initially identified group of intermediate ties was carried out.

There has been a lack in research to properly categorise weak, intermediate and strong ties in social networks, particularly when having more indicators for tie strength. The solution provided by this thesis is related to Petróczi et al.'s (2007) classification of tie strength in a network with several tie strength indicators. 
However, their research particularly explored tie strength within an organisational, but not inter-organisational context. Further, they did not use reciprocity as a second criterion to define the group of intermediate ties.

Although previous literature identified these intermediate ties (e.g. Granovetter, 1973; Dodds et al., 2003; Onnela et al., 2007), the studies focussed on social relationships at the individual level, and some studies (e.g. Granovetter, 1973) rather neglected the importance of these intermediate ties.

The important roles of intermediate ties in social networks were described in this thesis. Besides their numerous appearances in this study's social networks, the intermediate ties represented two specific roles. Their significance was represented by either Role 1, linking otherwise weakly linked groups of organisation to the network, or Role 2, linking two otherwise very strongly connected groups of organisation (key players). Therefore, intermediate ties might help receive new knowledge from outside the network with the help of Role 1 ties and also help access the local networks of other strongly connected network members with the help of Role 2 ties.

A recommendation on the most suitable SNA centrality measures in knowledge networks (dependent on the type of knowledge transferred)

This study identified Information Centrality as the most suitable SNA centrality measurement due to the types of knowledge most often transferred in the small-world networks investigated. Selecting the appropriate centrality measure for a particular network context can be cumbersome and measures in empirical network analysis have largely been misapplied (Borgatti, 2005). The appropriate centrality measure for a knowledge network is dependent on the type of knowledge transferred. In addition, it is important to consider how the knowledge is typically transferred in the network (Borgatti, 2005).

In this study's three small-world networks, formal factual knowledge as well as references to other sources of knowledge (meta-knowledge) were the more important 
electronically available types of knowledge transferred. As they were electronically available, they represented replicable types of knowledge. Although people often knew whom to contact for advice in the first place, it could not be assumed that every knowledge transfer is straightforward. In regard to this knowledge transfer and the replicable types of knowledge, Information Centrality was considered the most suitable SNA measurement for centrality. It also takes the characteristics of information/knowledge into account which includes the fact that knowledge does not necessarily follow the shortest path in an advice network. Information Centrality also considers different levels of tie strength among the groups of organisation, which appeared significant in this study.

In regard to electronically unavailable knowledge, such as books and documents, however, flow betweenness centrality is recommended as the most suitable measure. Flow betweenness considers transfer rather than replication of knowledge. In contrast to a replication, a transferred object does not remain with the sender like used goods, money or a package (Borgatti, 2005). As books and larger documents might be borrowed rather than copied, this type of knowledge would suggest transfer rather than replication.

In regard to electronically unavailable knowledge, such as tacit knowledge, Information Centrality can also be recommended, as tacit knowledge also represents a replicable type of knowledge (although different from the electronic available type).

Moreover, a relation could not be identified between the types of knowledge transferred and tie strength levels in regard to all three cases. However, some relation might exist as outlined in the case results in Chapters 4-6. Thus this study's findings would support Reagans and McEvily's (2003) argument that tie strength does not seem to be the main indicator for effective transfer of complex knowledge in comparison to Hansen's findings (1999). 


\section{Summary}

In summary, these contributions add to the body of literature investigating knowledge networks with SNA, particularly in the less researched inter-organisational knowledge transfer context in the R\&D sector (Allen et al., 2007). As the concepts of SNA still present a relatively new theoretical development, this thesis added knowledge to the application and further development of SNA concepts in an interorganisational knowledge transfer context.

In the wider $\mathrm{KM}$ context, this research addressed an acknowledged gap and emerging key theme in KM. Successful knowledge transfer is seen as being vital to organisational versatility, innovation and competitiveness. In the context of dynamic industrial cooperation, such as in R\&D networks, successful inter-organisational knowledge transfer in social relations also plays a key role for organisations. Despite this, there is almost no research into how knowledge transfer occurs in these formal and informal research and development networks. Thus social relations and organisational boundaries have been identified as two of six key emerging themes in the KM discipline into which further research is required (Argote et al., 2003).

\subsubsection{Practitioner value of the research}

From a practical perspective, this research aimed to add knowledge on how to increase collaboration among R\&D organisations in New Zealand. R\&D key players, such as CRIs, private research organisations, universities, government departments, and industry organisations, need to collaborate more in order to increase commercialisation of research knowledge, reduce costs (e.g. reuse knowledge) and drive innovation. This has been identified by the Ministry of Research, Science and Technology and Business New Zealand (Business NZ, 2006).

In addition, information about the key players, gained through an analysis of the levels of interactions in the networks, might be meaningful information providing 
managerial implications for future business decisions such as starting new projects or inter-organisational learning initiatives. In all three networks, CRIs, local government and private businesses played the key roles. In Cases 1 and 3 in particular, CRIs, local government and/or private businesses played the role of knowledge brokers. In Case 2 , central government and universities also had a powerful role for coordinating and controlling knowledge transfer in the network.

Moreover, knowledge about the study participants' preferred media characteristics and preferred ICT-based knowledge services for learning in the workplace may provide value to practitioners, especially when considering the introduction of supportive ICT.

\subsection{Directions for future research}

Several opportunities for future research have been recognised. They relate to either the study context, the research design, structural properties in knowledge networks or theory.

\section{Study context and research design}

As this study investigated the overall informal knowledge transfer among organisations at the workplace, future research could look at this kind of interorganisational knowledge transfer within project teams or particular business processes. In addition, an international context or a more long-term exploration of knowledge transfer in inter-organisational social networks would both represent other important dimensions.

Moreover, for a comparison of the key findings of this study, investigations in other $\mathrm{R} \& \mathrm{D}$ contexts could be useful, for example in regard to the identified network 
structures. In this regard, hypotheses could then be developed and tested to achieve more generalisable results.

This study is seen as preceding other studies that might measure the effectiveness/success of knowledge transfer supported by ICT and investigate the resulting communication and knowledge transfer structures. In terms of research design, a follow-up study could be conducted applying Action Research to implement a recommended ICT solution to support inter-organisational knowledge transfer and learning. As such a process would need to be done in several cycles including specific design and implementation stages, Action Research might be ideal to investigate such a scenario. Furthermore, success factors could be explored and the impact of ICT to support effective knowledge transfer could then be measured.

In addition, the ICT recommendations given in Chapter 8 could be the starting point for another study that aims to challenge and test these propositions in other contexts.

\section{Structural properties in knowledge networks}

As outlined, this study considered contact frequency, reciprocity (advice-giving and seeking) and the number of study participants from each organisation as indicators of tie strength. However, in the context of KM and knowledge transfer, the amount of time spent transferring/communicating knowledge as an additional tie strength indicator could be a further topic of interest. For example, tacit/non-codified knowledge may take more time than explicit knowledge to be transferred. In this regard, people in ties that spend more time would be more strongly connected than others spending less time.

Following up on the development of a definition of intermediate ties in knowledge networks, another k-means clustering approach could include the criterion 'reciprocity' as a second variable besides total tie strength values. This would allow for a single clustering approach using two variables at the same time. However, such a classification into weak, intermediate and strong ties could be integrated into tools 
for SNA such as UCINET. This would enable a more robust and quicker identification of the three different tie strength levels in knowledge networks.

\section{Theory-related directions}

Following up on the conclusion and discussion of the cross-case analysis results in Section 7.6, only two out of the three more important media capabilities for study participants in this study are related to Media Synchronicity Theory (MST). These were quick transfer of knowledge (transmission velocity and reuse of knowledge (reprocessability). Nevertheless, other media capabilities such as (perceived) ease of use appeared to be important too and might represent a combination of several other characteristics (e.g. quick transfer and reuse of knowledge). In regard to this consideration, an addition of this media characteristic to the set of media capabilities currently considered in MST would be suggested.

Moreover, another media capability particularly important to the inter-organisational knowledge transfer context is currently not considered in MST: the capability of providing a trustworthy environment which includes secure transfer of data. This seems an essential characteristic for communication in groups if group members come from diverse organisation and countries.

In regard to Wenger and Ferguson's (2006) knowledge ecology model, future research could also investigate a redesign of this model according to the assignment of characteristics such as guiding, participating, exploring and informing to suggested knowledge services such as online interaction with experts. 


\subsection{Limitations of this research}

While the findings of the research provide considerable value, there were also limitations which need to be recognised. These limitations include the research context, the study design and the focus on knowledge transfer and ICT support in social networks.

\section{Limitations of the research context}

This study aimed to explore particular knowledge transfer structures and processes in three inter-organisational social networks within a larger R\&D network in the environmental sector in New Zealand. Thus, the location for data collection was limited to New Zealand. In addition, as three social networks were explored by multiple case study approach, this thesis was limited to a group of participating individuals from diverse organisations. The number of participants was moreover limited to individuals who were members of an associated professional society that was contacted by the researcher for survey distribution in each case. Members were free to participate. However, due to the applied SNA approach, the results appear transferable to some extent. This may include other R\&D networks in New Zealand and other OECD countries.

\section{Limitations in regard to the study design}

This study developed an online survey with the help of a previous qualitative phase and employed typical SNA questions in the online survey to collect data on the contact frequency among organisations. Nevertheless, the survey design was limited in the final analysis in some ways. For example, no direct relation between preferred types of knowledge (or media characteristics) and associated types of relationships could be analysed. Although the analysis was possible at a broader level (strong and weak relationships), no specific types of knowledge or media characteristics could be identified, for example, for a CRIs - private businesses relationship. Additional

questions, however, which could have facilitated such an analysis, would have resulted in a longer survey. This could have constrained user-friendliness and therefore participation numbers. For future research, the relationship between the 
types of knowledge transferred in social relationships among specific groups of organisation could be investigated by another study using an additional survey. For example, this could reveal specific types of knowledge transferred in horizontal or vertical business relationships.

Due to the extended mixed methods approach of this study, the scope needed to be constrained in some places, for example when focussing on a few key themes in Phase 3, such as the use of social software/social networking tools by study participants, their preferred media characteristics and knowledge services, types of relationships and the identification of examples of the types of knowledge transferred. As Phase 3 was only conducted for Cases 2 and 3 and other themes such as the use of other ICT options or questions regarding why certain types of knowledge or media characteristics were preferred were not considered, this is another limitation of the study. Nevertheless, the selected key themes for Phase 3 helped explain and confirm the study results of Phase 2.

\section{Limitations of the focus on knowledge transfer and ICT support in social networks}

The study focussed on the identification of inter-organisational knowledge transfer structures and processes, but it did not aim to develop an instrument for measuring the effectiveness of this knowledge transfer. Moreover, the identification of specific knowledge transfer structures provided background data to identify possible ICT support for the three social networks. This study aimed to identify ICT to support knowledge transfer, but the research was not investigating IT adoption or technology acceptance. Implications for ICT support had the character of practical recommendations and were provided in Chapter 8 .

These limitations, regarding the study context, research design and the nature of knowledge transfer and ICT support in social networks, may, in addition to the outlined possibilities for future research in Section 9.4, also provide indications for future research. 


\subsection{Chapter summary}

In this concluding chapter, an overview of the research, including context, gaps in the literature, research questions, research design and key findings, was provided. The contributions of the research were then described, both with regard to the academic value of the research and the practitioner value. Implications for future research were outlined. Finally, the limitations of the research relating to the context, the research design and the nature of knowledge transfer in social networks were described. 


\section{References}

Adams, J. S. (1976). The structure and dynamics of behavior in organizational boundary roles. In M. D. Dunnette (Ed.), Handbook of Industrial and Organizational Psychology (pp. 1175-1199). Chicago: Rand McNally.

Adler, P., \& Kwon, S. W. (2002). Social capital: prospects for a new concept. Academy of Management Review, 27, 17-40.

Ahuja, M., \& Carley, K. (1999). Network structure in virtual organizations. Organization Science, 10(6), 741-757.

Albert, R., \& Barabasi, A. (2000). Topology of evolving networks: Local events and universality. Physical Review Letter, 85(24), 5234-5237.

Aldrich, H., \& Herker, D. (1977). Boundary-spanning roles and organization structure. Academy of Management Review, 2, 217-230.

Allen, J., James, A. D., \& Gamlen, P. (2007). Formal versus informal knowledge networks in R\&D: a case study using social network analysis. $R \& D$ Management, 37(3), 179-196.

Allen, T. J. (1977). Managing the Flow of Technology. Cambridge, MA: MIT Press.

Amin, A., \& Cohendet, P. (2004). Architectures of knowledge: firms, capabilities and communities. . Oxford: Oxford University Press.

Anderson, T. (2005, 9-11 November). Distance learning - social software's killer ap? Paper presented at the 17th Biennial Conference of the Open and Distance Learning Association of Australia (ODLAA), Adelaide, South Australia.

Anderson, T., \& Kanuka, H. (2003). e-Research Methods, Strategies and Issues. Boston: Allyn and Bacon.

Ang, S., \& Slaughter, S. (2001). Work Outcomes and Job Design for Contract Versus Permanent Information: Systems Professionals on Software Development Teams MIS Quarterly, 25(3), 321-350.

Anklam, P. (2002, 29-31 October ). Social Network Analysis for Knowledge Management. Paper presented at the KMWorld, Santa Clara, California.

Argote, L., McEvily, B., \& Reagans, R. (2003). Managing Knowledge in Organizations: An Integrative Framework and Review of Emerging Themes. Management Science, 49(4), 571-582. 
Aslesen, H. W. (2005, 18-21 May). Commercialisation of Knowledge. The case of Norwegian Marine Biotechnology. Paper presented at the 5th Triple Helix Conference "The capitalization of knowledge:cognitive, economic, social \& cultural aspects", Turin-Milan, Italy.

Atallah, G. (2000). Research Joint Ventures Cartelization with Asymmetric R\&D Spillovers. Economics Bulletin, 12(18), 1-11.

Badaracco, J. L. (1991). Alliances speed knowledge transfer. Strategy \& Leadership, 19(2), 10-16.

Baum, J. A., Rowley, T. J., \& Shipilov, A. V. (2004). The Small World of Canadian Capital Markets: Statistical Mechanics of Investment Bank Syndicate Networks, 1952-1989. Canadian Journal of Administrative Sciences, 21(4), $307-325$.

Becerra, M., Lunnan, R. \& Huemer, L. (2008). Trustworthiness, Risk, and the Transfer of Tacit and Explicit Knowledge Between Alliance Partners. Journal of Management Studies, 45(4), 691-713.

Benbasat, I., Goldstein, D. K., \& Mead, M. (1987). The Case Research Strategy in Studies of Information Systems. MIS Quarterly, 11(3), 369-386.

Best, S. J., \& Krueger, B. S. (2004). Internet data collection. Thousand Oaks, California, USA: Sage Publications, Inc.

Boisot, M. (1987). Informations and Organisations: The Manager as Anthropologist. London: Fontana/Collins.

Bonacich, P. (1972). Factoring and Weighting Approaches to Status Scores and Clique Identification. Journal of Mathematical Sociology, 2, 113-120.

Bonk, C. J., \& Graham, C. R. (2006). The handbook of blended learning: global perspectives, local designs (1 ed.). San Francisco: Pfeiffer.

Borgatti, S. P. (1995). Centrality and AIDS. Connections, 18(1), 112-114.

Borgatti, S. P. (2005). Centrality and Network Flow. Social Networks, 27, 55-71.

Borgatti, S. P., Everett, M. G., \& Freeman, L. C. (2002). Ucinet for Windows: Software for Social Network Analysis. Harvard, MA: Analytic Technologies.

Borgatti, S. P., \& Molina, J. L. (2003). Ethical and strategic issues in organizational social network analysis. The Journal of Applied Behavioural Science, 39(3), 337-349. 
Borrego, M., Osborne, L., Streveler, R., Smith, K., \& Miller, R. (2007). Quantitative and qualitative measures of community development through a structured workshop curriculum. West Lafayette, Indiana: American Society for Engineering Education.

Branscomb, L. M. (1992). Information Infrastructure for the 1990's: A Public Policy Perspective. In B. Kahin (Ed.), Building Information Infrastructure: Issues in the Development of the National Research and Education Network (pp. 15 30). New York: McGraw-Hill.

Bresman, H., Birkinshaw, J., \& Nobel, R. (1999). Knowledge transfer in international acquisitions. Journal of International Business Studies, 30(3), 439-462.

Brogan, M., \& Armstrong, L. (2005, 28-29 November). C-commerce innovation: unraveling the effects of knowledge ties on embedded network structure. Paper presented at the KMAP, Wellington, New Zealand.

Brown, J. S., \& Duguid, P. (1998). Organizing Knowledge. Reflections, 1(2), 28-44.

Burgoon, J. K., Bonito, J. A., Bengtsson, B., Ramirez Jr., A., Dunbar, N. E., \& Miczo, N. (1999-2000). Testing the Interactivity Model: Communication Processes, Partner Assessments, and the Quality of Collaborative Work. Journal of Management Information Systems, 16(3), 33-56.

BusinessNZ. (2006). More collaboration needed between business and researchers. Retrieved 11 August 2007, 2007, from http://www.businessnz.org.nz/doc/1109/Morecollaborationneededbetweenbus inessandresearchers

Calloway, M., Morrissey, J. P., \& Paulson, R. I. (1993). Accuracy and reliability in self-reported data in interorganizational networks. Social Networks, 15, 377398.

Carayannis, E. G. (1999). Fostering synergies between information technology and managerial and organizational cognition: the role of knowledge management Technovation, 19(4), 219-231.

Carlile, P., \& Rebentisch, E. (2003). Into the black box: the knowledge transformation cycle. Management Science, 49(9), 1180-1195.

Carr, A. (2000). Commercialisation of scientific research : spin-off companies from Industrial Research. Unpublished Masters thesis, Victoria University of Wellington, Wellington.

Carrington, P. J., Scott, J., \& Wasserman, S. (2005). Models and methods in social network analysis. New York: Cambridge University Press. 
Chan, K., \& Liebowitz, J. (2006). The synergy of social network analysis and knowledge mapping: a case study. International Journal of Management and Decision Making, 7(1), 19-35.

Chaturvedi, A., Carroll, J. D., Green, P. E., \& Rotondo, J. A. (1997). A feature-based approach to market segmentation via overlapping K-centroids clustering. Journal of Marketing Research, 34, 370-377.

Chaudhury, A., \& Agrawal, M. (2002). Business modelling: multidisciplinary approaches, economics, operational, and information systems perspectives : in honor of Andrew B. Whinston. Norwell, Massachusetts: Kluwer Academic Publishers.

Chawner, B. (2008). Spectators, not players: information managers' use of Web 2.0 in New Zealand. The Electronic Library, 26(5), 630 - 649.

Chen, X., \& Wang, L. (2009). Integrating Biological Knowledge with Gene Expression Profiles for Survival Prediction of Cancer. Journal of Computational Biology, 16(2), 265-278.

Cho, H. (2002). Analyzing electronic learning communities using social network analysis. Cornell University, New York.

Cho, H. K., Trier, M., \& Kim, E. (2005). The Use of Instant Messaging in Working Relationship Development: A Case Study Journal of Computer Mediated Communication, 10(4), Article 17.

Connell, C., Klein, J. H., \& Powell, P. (2003). It's tacit knowledge but not as we know it:redirecting the search for knowledge. Journal of the Operational Research Society, 54, 140-152.

Connell, T. (1995). Subject Searching in Online Catalogs: Metaknowledge Used by Experienced Searchers. Journal of the American Society for Information Science 46(7), 506-518.

Cowan, R. (2004). Network models of innovation and knowledge diffusion. Maastricht, The Netherlands: MERIT - Maastricht Economic Research Institute on Innovation and Technology, International Institute of Infonomics.

Cowan, R., \& Jonard, N. (2004). Network structure and the diffusion of knowledge Journal of Economic Dynamics and Control, 28(8), 1557-1575.

Crabtree, B. F., \& Miller, W. L. (2000). Using Codes and Code Manuals. In Doing Qualitative Research (2nd ed.). Thousand Oaks: CA: Sage Publications. 
Cranefield, J., \& Yoong, P. (2005). Stages of inter-organisational knowledge transfer: an exploratory study of the New Zealand State Sector. International Journal of Knowledge, Culture and Change Management, 5(5), 75-84.

Cranefield, J., \& Yoong, P. (2010). Knowledge Broker in Overlapping Online Communities of Practice: The Role of the Connector-Leader. In P.Yoong(Ed.), Leadership in the Digital Enterprise (pp. 77-90). Hershey, PA: IGI Global.

Creswell, J. W. (2003). Research Design:Qualitative, Quantitative and Mixed Methods Approaches (2nd ed.). Thousands Oaks, CA: Sage.

Creswell, J. W. (2007). Research Design: Qualitative, quantitative and mixed methods approaches. Thousand Oaks: Sage.

Cross, R., Borgatti, S. P., \& Parker, A. (2001). Beyond answers: dimensions of the advice network Social Networks, 3, 215-235.

Cross, R., Borgatti, S. P., \& Parker, A. (2002a). Making invisible work visible: using social network analysis to support strategic collaboration. California Management Review, 44(2), 25-46.

Cross, R., Laseter, T., Parker, A., \& Velasquez, G. (2006). Using Social Network Analysis to Improve Communities of Practice. California Management Review, 49(1), 32-60.

Cross, R., Nohria, N., \& Parker, A. (2002b). Six myths about informal networks - and how to overcome them. MIT Sloan Management Review, 43(3), 66-75.

Cross, R., \& Parker, A. (2004). The Hidden Power of Social Networks. Bosten: Harvard University Press.

Cross, R., \& Prusak, L. (2002). The people who make organizsations go or stop. Harvard Business Review, 80(6), 104-112.

Cummings, J. L. (2001). Knowledge Transfer across R\&D units: An Empirical Investigation of the Factors Affecting Successful Knowledge Transfer Across Intra- and Interorganizsational Units. George Washingtion University, Washingtion, DC.

Daft, R. L., \& Lengel, R. H. (1984). Information Richness: A New Approach to Managerial Behaviour and Organizational Design. In L. Cummings \& B. Staw (Eds.), Research in Organizational Behaviour (pp. 191-233). Homewood, IL: JAI Press. 
Dalsgaard, C. (2006). Social software: E-learning beyond learning management systems [Electronic Version]. European Journal of Open, Distance and Elearning. Retrieved 10 May 2009 from http://www.eurodl.org/materials/contrib/2006/Christian_Dalsgaard.htm.

Davenport, T., \& Prusak, L. (1998). Working knowledge: How organizsations manage what they know, Bosten, MA (USA): Harvard Business School Press.

Davis, F.D. (1989). Perceived Usefulness, Perceived Ease of Use, and User Acceptance of Information Technology, MIS Quarterly, 13(3), 319-339.

Delphi. (1997). Delphi On Knowledge Management. Research \& Perspectives on Today's Knowledge Landscape. Bosten, MA (USA): The Delphi Consulting Group.

DeLuca, D., \& Valacich, J. S. (2006). Virtual teams in and out of synchronicity. Information Technology \& People, 19(4), 323 - 344.

Dennis, A. R., Fuller, R. M., \& Valacich, J. S. (2008). Media, tasks, and communication processes: a theory of media synchronicity. MIS Quarterly, 32(3), 575-600.

Dennis, A. R., \& Valacich, J. S. (1999, 5-8 January). Rethinking Media Richness: Towards a Theory of Media Synchronicity. Paper presented at the 32nd Hawaii International Conference on System Sciences, Hawaii.

Denzin, N. K., \& Lincoln, Y. S. (1994). Introduction: Entering the field of qualitative research. In N. K. Denzin \& Y. S. Lincoln (Eds.), Handbook of qualitative research (pp. 1-17). Thousand Oaks, CA: Sage.

Despres, C., \& Chauvel, D. (2000). Knowledge horizons. Oxford: ButterworthHeinemann.

Dodds, P. S., Muhamad, R., \& Watts, D. J. (2003). An Experimental Study of Search in Global Social Networks. Science, 301(5634), 827 - 829.

Dreyfus, H., \& Dreyfus, S. (1986). Mind over machine. New York: Free Press.

Dubé, L., \& Paré, G. (2003). Rigor in Information Systems Positivist Case Research:Current Practices, Trends and Recommendations. MIS Quarterly, 27(4), 597-636.

Easterby-Smith, M., Lyles, M., \& Tsang, E. (2008). Inter-organizational Knowledge Transfer: Current Themes and Future Prospects. Journal of Management Studies, 45(4), 677-690. 
Eisenhardt, K. (1989). Building theories from case study research. Academy of Management Review, 14(4), 532-550.

Erdos, P., \& Renyi, A. (1960). On the evolution of random graphs. Publications of the Mathematical Institute of the Hungarian Academy of Sciences, 5(A), 1761.

Everett, M., \& Borgatti, S. P. (2008, 22-27 January ). Giving and Receiving Centrality. Paper presented at the Sunbelt XXVIII International Sunbelt Social Network Conference, St. Pete Beach, FL.

Ferligoj, A., \& Hlebec, V. (1999). Evaluation of social network measurement instruments. Social Networks, 21, 111-130.

Forment, M. A. (2007). A Social Constructionist Approach to Learning Communities: Moodle. In M. Lytras \& A. Naeve (Eds.), Open Source for Knowledge and Learning Management: Strategies Beyond Tools (pp. 369381). Hershey, PA: Idea Group Publishing.

Foster-Fishman, P. G., Salem, D. A., \& Allen, N. A. (2001). Facilitating Interorganizational Collaboration: The Contributions of Interorganizational Alliances. American Journal of Community Psychology, 29(6), 875-905.

Freeman, L. C. (1979). Centrality in social networks: Conceptual clarification. Social Networks, 1, 215-239.

Freeman, L. C., Borgatti, S. P., \& White, D. R. (1991). Centrality in valued graphs: a measure of betweenness based on network flow. Social Networks, 13, 141154.

Friedkin, N. (1980). A Test of Structural features of Granovetter's Strength of weak Ties Theory. Social Networks, 2, 411-422.

Fritsch, M., \& Franke, G. (2004). Innovation, regional knowledge spillovers and R\&D cooperation. Research Policy, 33(2), 245-255.

Galliers, R. D. (1992). Choosing information systems research approaches. In R. D. Galliers (Ed.), Information Systems Research: Issues, methods and practical guidelines (pp. 144-162). Oxford: Blackwell Scientific.

Galliers, R. D. (1993). Research issues in information systems. Journal of Information Technology 8(2), 92-98

Garton, L., Haythornthwaite, C., \& Wellman, B. (1997). Studying online social networks [Electronic Version]. Journal of Computer Mediated Communication, 3. Retrieved 10 May 2009 from http://jcmc.indiana.edu/vol3/issue1/garton.html. 
Goodhue, D., \& Thompson, R. (1995). Task-technology-fit and individual performance. MIS Quarterly, 19 (2), 213-236.

Graham, C. R. (2006). Blended Learning Systems: Definition, Current Trends, and Future Directions. In C. J. Bonk \& C. R. Graham (Eds.), The Handbook of Blended Learning (pp. 3-21). San Francisco: Pfeiffer Publishing.

Granovetter, M. (1973). The Strength of Weak Ties. American Journal of Sociology, $78(6), 1360-1380$.

Granovetter, M. (1974). Getting a job: A study of contacts and careers (1 ed.). Cambridge, Massachusetts: Harvard University Press.

Granovetter, M. (1995). Getting a job: A study of contacts and careers (2 ed.). Chicago: The University of Chicago Press.

Griliches, Z. (1991). The search for knowledge spillovers. Cambridge, MA: National Bureau of Economic Research.

Guba, E. G., \& Lincoln, Y. S. (1994). Competing paradigms in qualitative research. In N. K. Denzin \& Y. S. Lincoln (Eds.), Handbook of qualitative research (pp. 105-117). London: Sage.

Hanneman, R. (2005). Introduction to social network methods. Retrieved 7 July, 2007, from http://www.faculty.ucr.edu/

Hansen, M. T. (1999). The Search-Transfer Problem: The Role of Weak Ties in Sharing Knowledge across Organisational Subunits. Administrative Science Quarterly, 44(1), 82-111.

Hansen, M. T., Nohria, N., \& Tierney, T. (1999). What's your strategy for managing knowledge? Harvard Business Review, 77(3-4), 106-116.

Hara, N., \& Schwen, T. M. (2006). Communities of Practice in Workplaces. Performance Improvement Quality, 18(2), 93-114.

Harryson, S. J., Dudkowski, R., \& Stern, A. (2008). Transformation Networks in Innovation Alliances - The Development of Volvo C70. Journal of Management Studies, 45(4), 745-773.

Hayek, F. A. (1945). The Use of Knowledge in Society. The American Economic Review, 35(4), 519-530.

Haythornthwaite, C. (1996). Media use in support of communication networks in an academic research environment. University of Toronto, Toronto. 
Haythornthwaite, C. (1996). Social network analysis:An approach and technique for the study of information exchange. Library and Information Science Research, 18, 323-342.

Haythornthwaite, C. (1999). A Social Network Theory of Tie Strength and Media Use: A Framework for Evaluating Multi-level Impacts of New Media (No. Technical Report UIUCLIS--2002/1+DKRC). Champaign, IL: Graduate School of Library and Information Science, University of Illinois at UrbanaChampaign.

Haythornthwaite, C. (2005). Social Netwoks and Internet Connectivity Effects. Information, Communication \& Society, 8(2), 125-147.

Haythornthwaite, C., \& Wellman, B. (1998). Work, friendship, and media use for information exchange in a networked organization. Journal of the American Society for Information Science, 49(12), 1101 - 1114.

Herriot, R. E., \& Firestone, W. A. (1983). Multisite qualitative policy research: optimizing description and generalizability. Educational Researcher, 12(2), 14-19.

Hexmoor, H., Wilson, S., \& Bhattaram, S. (2006). A theoretical inter-organizational trust-based security model. The Knowledge Engineering Review, 21(2), 127161.

Hinds, P., \& Kiesler, S. (1995). Communication across boundaries: Work, structure, and use of communication technologies in a large organization. Organization Science, 6(4), 373-393.

Hirschheim, R., \& Klein, H. K. (1989). Four paradigms of information systems development. Communications of the ACM, 32(10), 1199 - 1216.

Holsapple, C. W., \& Jones, K. (2004). Exploring Primary Activities of the Knowledge Chain. Knowledge and Process Management, 11(3), pp 155-174.

Hooper, V. (2006). The Impact of the Alignment between Information Systems and Marketing on Business Performance. Victoria University of Wellington, Wellington.

Huberman, A. M., \& Miles, M. B. (1998). Data management and analysis methods. In N. K. Denzin \& Y. S. Lincoln (Eds.), Collecting and interpreting qualitative materials (pp. 179-210). London: Sage.

Infocent package:sna R Documentation: Find Information Centrality Scores of Network Positions Description.(2009).Retrieved May 24, 2009, from http:// genome.jouy.inra.fr/doc/bioinfo/statistiques/R2.6.0/library/sna/help/infocent 
Jewels, T., Underwood, A., Gregor, S., \& de Pablos, C. (2004, 8-11 July). Motivators and Inhibitors for Managing IT Project Knowledge: Findings from Three Exploratory Case Studies. Paper presented at the 8th Pacific Asia Conference on Information Systems, Shanghai, China.

Johnson, R. B., \& Onwuegbuzie, A. J. (2004). Mixed Methods Research: A Research Paradigm Whose Time Has Come Educational Researcher, 33(7), 14-26.

Jonkeren, E., \& Huysman, M. (2006, 20-22 March). Identifying informal knowledge networks through SNA, revealing the stickiness of communities. Paper presented at the International Conference on Organizational Learning, Knowledge and Capabilities University of Warwick, Conventry.

Kaplan, B., \& Duchon, D. (1988). Combining Qualitative and Quantitative Methods in Information Systems Research: A Case Study MIS Quarterly, 12(4), 571586.

Kaplan, B., \& Maxwell, J. A. (1994). Qualitative Research Methods for Evaluating Computer Information Systems. In J. Anderson, C. Aydin \& S. Jay (Eds.), Evaluating Health Case Information Systems: Methods and Applications (pp. 45-68). California: Sage.

Kapyla, T., Niemi, I., \& Lethola, A. (1998, 19-21 October). Towards an Accessible Web by Applying Push Technology. Paper presented at the Proc.Fourth ERCIM Workshop on "User interfaces for all", Stockholm, Sweden.

Karlsson, C. P., Flensburg.S.A., \& Hoerte. (2004). Knowledge spillovers and Knowledge Management. Chettenham: Edward Elgar.

Kelle, U. (2006). Combining qualitative and quantitative methods in research practice: purposes and advantages. Qualitative Research in Psychology, 3, 293-311.

Kendall, J. E., \& Kendall, K. E. (1999). Information Delivery Systems: an exploration of web pull and push technologies. Communication of the AIS, 1(4), Article No.1.

Kerres, M., \& De Witt, C. (2003). A Didactical Framework for the Design of Blended Learning Arrangements. Learning, Media and Technology, 28(2/3), 101-113.

Klein, H., \& Myers, M. (1999). A set of principles for conducting and evaluating interpretive field studies in information systems. MIS Quarterly, 23(1), 67-93.

Koschatzky, K. (2004). The role of R\&D services in managing regional knowledge generation - a regional differentiation. In C. Karlsson, P. Flensburg \& S.-A. Hoerte (Eds.), Knowledge Spillovers and Knowledge Management. Northampton, Massachussets: Edward Elgar Publishing. 
Krackhardt, D., \& Hanson, J. R. (1993). Informal Networks: The Company Behind the Chart. Harvard Business Review, 71(4), 104-111.

Kratzer, J., Gemünden, H. G., \& Lettl, C. (2008). Balancing Creativity and Time Efficiency in Multi-Team R\&D Projects: The Alignment of Formal and Informal Networks R\&D Management, 38(5), 538-549.

Krefting, L. (1991). Rigor in Qualitative Research: The Assessment of Trustworthiness. The American Journal of Occupational Therapy, 45(3), 214222.

Kupperschmidt, B. R. (2001). Understanding Net Generation Employees. The Journal of Nursing and Administration, 31(12), 570-574.

Kwan, M. M., \& Cheung, P.-K. (2006). The Knowledge Transfer Process: From Field Studies to Technology Development. Journal of Database Management, 17(1), 16-32.

Kwon, D., Oh, W., \& Jeon, S. (2007). Broken Ties: The Impact of Organizational Restructuring on the Stability of Information-Processing Networks. Journal of Management Information Systems, 24(1), 201-231.

Lamb, J. (2001, 6 June). "Blended Learning" is the new buzz phrase. Financial Times.

Langlois, S. (1977). Les Réseaux Personnels et la Diffusion des Informations sur les Emplois. Recherches Sociographiques, 2, 213-245.

Lee, A. S. (1989). A Scientific Methodology for MIS Case Studies. MIS Quarterly, 13(1), 33-52.

Lehmann, H., \& Lehner, F. (2005). Holistic perspectives of information sharing and knowledge exchange: selected frameworks and their use. International Journal of Information Technology and Management (IJITM), 4(3), 290-307.

Leskovec, J., \& Horvitz, E. (2008, April). Planetary-Scale Views on a Large InstantMessaging Network. Paper presented at the WWW 2008, Beijing, China.

Levin, D. Z., \& Cross, R. (2004). The strength of weak ties you can trust: The mediating role of trust in effective knowledge transfer. Management Science, 50(11), 1477-1490.

Liebowitz, J. (2005). Linking social network analysis with the analytic hierarchy process for knowledge mapping in organizations. Journal of Knowledge Management, 9(1), 76-86. 
Lin, N., Dayton, P. W., \& Greenwald, P. (1978). Analyzing the Instrumental Use of Relations in the Context of Social Structure. Sociological Methods and Research, 7, 149-166.

Lincoln, Y., \& Guba, E. (1985). Naturalistic Inquiry. Beverly Hills, CA: Sage Publications.

Linstone, H. A., \& Turoff, M. (1975). The Delphi Method. Techniques and Applications. London, UK: Addison-Wesley.

Lipp, M. (2006). VPN - virtuelle private Netzwerke - Aufbau und Sicherheit. Munich: Addison-Wesley Verlag.

Lumsden, L., Breathnach, C., \& Richards, D. (2003, 23 November). Using UCINET 6 to develop a questionnaire to collect weighted relationship data. Paper presented at the 34th Annual PSI Conference, Limerick.

Maier, R. (2001). Knowledge Management Systems (1 ed.). Berlin, Heidelberg: Springer.

Maier, R., \& Hädrich, T. (2006). Centralized Versus Peer-to-Peer Knowledge Management Systems. Knowledge and Process Management, 13(1), 47-61.

Maier, R., Haedrich, T., \& Peinl, R. (2005). Enterprise knowledge infrastructures Berlin: Springer Verlag.

Majchrzak, A., Cooper, L. P., \& Neece, O. E. (2004). Knowledge Reuse for Innovation. Management Science, 50(2), 174-188.

Markus, L. M. (2001). Toward a Theory of Knowledge Reuse: Types of Knowledge Reuse Situations and Factors in Reuse Success. Journal of Management Information Systems, 18(1), 57-93.

Markus, M. L. (1994). Electronic Mail As the Medium of Managerial Choice Organization Science, 5(4), 502-527.

Marsden, P. V., \& Campbell, K. E. (1984). Measuring Tie Strength. Social Forces, 63(2), 482-501.

Maruping, L. and Agarwal, R. (2004). Managing Team Interpersonal Processes Through Technology: A Task-Technology Fit Perspective. Journal of Applied Psychology, 89(6), 975-990.

Mason, K. J., \& Leek, S. (2008). Learning to Build a Supply Network: An Exploration of Dynamic Business Models. Journal of Management Studies, 45(4), 774-799. 
Mathews, K. M., White, M. C., Soper, B., \& Bergen, C. W. (1998). Association of indicators and predictors of tie-strength. Psychological Reports, 83, 14591469.

Matthews, P. (1999). Workplace learning: developing an holistic model. The Learning Organization, 6(1), 18.

McAdam, R., \& McCreedy, S. (1999). A critical review of knowledge management models. The Learning Organization, 6(3), 91-100.

McEvily, B., \& Zaheer, A. (1999). Bridging ties: a source of firm heterogeneity in competitive capabilities. Strategic Management Journal, 20(12), 1133 - 1156.

Miles, M. B., \& Huberman, A. M. (1984). Qualitative Data Analysis: A Sourcebook of New Methods. Thousand Oaks, CA: Sage.

Miles, M. B., \& Huberman, A. M. (1994). Qualitative Data Analysis: An Expanded Sourcebook (2nd ed.). Thousand Oaks,CA: Sage.

Mingers, J. (2001). Combining IS research methods:Towards a pluralist methodology. Information Systems Research, 12, 240-259.

Ministry of Research Science and Technology. (2006). Research and Development in New Zealand: A decade in review. Retrieved 10 May 2009. from http://www.mhttp://www.morst.govt.nz/publications/a-z/r/decade-in-review/report/.

Mitchell, V. L. (2006). Knowledge Integration and Information Technology Project Performance. MIS Quarterly, 30(4), 919-939.

Mizruchi, M. S., \& Marquis, C. (2006). Egocentric, sociocentric, or dyadic?: Identifying the appropriate level of analysis in the study of organizational networks Social Networks, 28(3), 187-208.

Mladenić, D., Grobelnik, M., Fortuna, B., \& Grćar, M. (2009). Knowledge Discovery for Semantic Web In J. Davies, M. Grobelnik \& D. Mladenić (Eds.), Semantic Knowledge Management (pp. 21-36). Berlin Heidelberg: Springer.

Moore, G., C., and Benbasat, I. (1991) Development of an Instrument to Measure the Perceptions of Adopting and Information Technology Innovation, Information Systems Reseaerch, 2(3), 192-222.

Moreira, A. C. (2009). Knowledge capability flows in buyer-supplier relationships: Challenges for small domestic suppliers in international contexts. Journal of Small Business and Enterprise Development, 16(1), 93-114.

Morgan, D. L. (2007). Paradigms Lost and Pragmatism Regained Journal of Mixed Methods Research, 1(1), 48-76. 
Mowery, D. C., Oxley, J. E., \& Silverman, B. S. (1996). Strategic Alliances and Interfirm Knowledge Transfer. Strategic Management Journal, 17(Winter Special Issue), 77-91.

Munkvold, B. E. (2003). Implementing Collaboration Technologies in Industry: Springer.

Munzer, S. and Holmer T. (2009). Bridging the Gap Between Media Synchronicity and Task Performance. Communication Research. 36(1), 76-103.

Murthy, U. S., \& Kerr, D. S. (2003). Decision Making Performance of Interacting Groups: An Experimental Investigation of the Effects of Task Type and Communication Mode. Information and Management, 40, 351-360.

National Board of Employment Education and Training (NBEET). (1993). Workplace Learning in the Professional Development of Teachers. Canberra: Australian Government publishing service.

Nguyen, V. K. (2006). Small-world graphs: Models, Analysis and Applications for Largescale Dynamic Networks. University of California, Davis.

Nonaka, I. (1991). The Knowledge-Creating Company. Harvard Business Review, 69(6), 96-104.

Nonaka, I. (1994). A Dynamic Theory of Organizational Knowledge Creation. Organization Science, 5(1), 14-37.

Nonaka, I., Toyama, R., \& Konno, N. (2000). SECI, Ba and Leadership: a Unified Model of Dynamic Knowledge Creation. Long Range Planning, 33(1), 5-34.

Nonaka, I., \& Krogh, G. (2009). Perspective-Tacit Knowledge and Knowledge Conversion: Controversy and Advancement in Organizational Knowledge Creation Theory. Organization Science, 20(3), 635-652.

Nonaka, I., \& Takeuchi, H. (1995). The Knowledge Creating Company: How Japanese Companies Create the Dynamics of Innovation. New York: Oxford University Press.

Nurmi, R. (1998). Knowledge-intensive firms.Business Horizons, 41(3), 26-32.

Onnela, J. P., Saramäki, J., Hyvönen, J., Szabó, G., Lazer, D., Kaski, K., et al. (2007). Structure and tie strengths in mobile communication networks. Proceedings of the National Academy of Sciences of the United States of America, 104(18), 7332-7336. 
Orlikowski, W., \& Baroudi, J. (1991). Studying Information Technology in Organizations: Research Approaches and Assumptions. Information Systems Research, 2(1), 1-28.

Paré, G. (2004). Investigating Information Systems with a Positivist Case Study. Research Communications of the AIS, 13, 244-264.

Patton, M. Q. (1990). Qualitative Evaluation and Research Methods (2nd ed.). Newbury Park, CA: Sage Publications.

Pauleen, D., \& Yoong, P. (2001). Relationship Building and the Use of ICT in Boundary-Crossing Virtual Teams: A Facilitator's Perspective. Journal of Information Technology, 16(4), 205-220.

Pawlowski, S. D., \& Robey, D. (2004). Bridging User Organizations: Knowledge Brokering and the Work of Information Technology Professionals. MIS Quarterly, 28(4), 645 - 672.

Pérez-Nordtvedt, L., Kedia, B. L., Datta, D. K., \& Rasheed, A. A. (2008). Effectiveness and Efficiency of Cross-Border Knowledge Transfer: An Empirical Examination. Journal of Management Studies, 45(4), 715-744.

Petróczi, A., Nepusz, T., \& Bazsó, F. (2007). Measuring tie-strength in virtual social networks. Connections, 27(2), 39-52.

Pickering, J. M., \& King, J. L. (1995). Hardwiring Weak Ties: Interorganizational Computer-mediated Communication, Occupational Communities, and Organisational Change. Organization Science, 6(4), 479-486.

Polanyi, M. (1966). The Tacit Dimension. Garden City, N.Y.: Doubleday.

Polites, G., \& Watson, R. (2008). The Centrality and Prestige of CACM. Communications of the ACM, 51(1), 95-100.

Porter, M. E. (2001, August, 3rd, 2001). New Zealand Competitiveness: The Next Agenda. Retrieved 16/1/2007, 2007.

Priestley, J. L. (2003). Inter-Organizational Knowledge Transfer Difficulty: The Influence of Organizational Network Type, Absorptive Capacity, Causal Ambiguity and Outcome Ambiguity. Georgia State University, Atlanta, Georgia.

Probst, G., Raub, S., \& Romhardt, K. (1998). Wissen managen: Wie Unternehmen ihre wertvollste Ressource optimal nutzen (2nd ed.). Wiesbaden: Gabler. 
Rabinowitz, V. C., \& Weseen, S. (1997). Elu(ci)d(at)ing epistemological impasses: Re-viewing the qualitative/quantitative debates in psychology. Journal of Social Issues, 53(4), 605-626.

Reagans, R., \& McEvily, B. (2003). Network structure and knowledge transfer: The effects of cohesion and range. Administrative Science Quarterly, 48, 240-267.

Ridenour, C. S., \& Newman, I. (2008). Mixed Methods Research: Exploring the Interactive Continuum. Carbondale, Southern Illinois: Southern Illinois University Press.

Riopelle, K., Gluesing, J., Alcordo, T., Baba, M., Britt, D., McKether, W., et al. (2003). Context, Task and the Evolution of Technology Use in Global Virtual Teams. In C. Gibson \& S. G. Cohen (Eds.), Virtual teams that work: Creating Conditions for Virtual Team Effectiveness. San Francisco, CA: Jossey-Bass.

Roberts, E. B. (1988). Managing Invention and Innovation. Research Technology Management, 31(1), 11-29.

Rogers, E. (1962). Diffusion of Innovations ( $1^{\text {st }}$ ed.). Glencoe: Free Press.

Rogers, E. (2003). Diffusion of Innovations (5th ed.). New York: The Free Press.

Rolland, N., \& Chauvel, D. (2000). Knowledge Transfer in Strategic Alliances. In C. Despres \& D. Chauvel (Eds.), Knowledge Horizons. Boston, Oxford, Auckland, Johannesburg, Melbourne, New Delhi: Butterworth Heinemann.

Rooney, J. E. (2003). Blending learning opportunities to enhance educational programming and meetings. Association Management, 55(5), 26-32.

Ruggles, R. L. (1998). The State of the Notion: Knowledge Management in Practice. California Management Review, 40(3), 80-89.

Sambamurthy, V., \& Subramani, M. (2005). Special Issue forword, Special issue on Information Technologies and Knowledge Management. MIS Quarterly, 29(1), 1-7.

Sammarra, A., \& Biggiero, L. (2008). Heterogeneity and Specificity of Inter-Firm Knowledge Flows in Innovation Networks. Journal of Management Studies, 45(4), 800-829.

Schilling, M. A., \& Phelps, C. C. (2007). Interfirm Collaboration Networks: The Impact of Small World Connectivity on Firm Innovation Management Science, 53(7), 1113-1126. 
Schmitz, C., Hotho, A., Jaeschke, R., \& Stumme, G. (2006). Kollaboratives Wissensmanagement. In T. Pellegrini, Blumauer, A. (Ed.), Semantic Web (pp. 273-290). Berlin Heidelberg: Springer.

Schoder, D., \& Fischbach, K. (2003). Peer-to-peer prospects. Communications of the $A C M, 46(2), 27-29$.

Schultze, U., \& Leidner, D. E. (2002). Studying knowledge management in information systems research: Discourse and theoretical assumptions. . MIS Quarterly, 26(3), 213-242.

Scott, J. (2000). Social Network Analysis: a handbook. London: Sage Publications Ltd.

Seale, C. (1999). Quality in qualitative research Thousand Oaks, CA: Sage.

Shahriza, N., Karim, A., \& Heckman, R. (2005). Group Communication Media Choice and the Use of Information and Communication Technology to Support Learning: A Case Study. Campus-Wide Information Systems, 22(1), $28-42$.

Simonin, B. L. (1999). Ambiguity and the process of knowledge transfer in strategic alliances. Strategic Management Journal, 20(7), 595 - 623.

Stankosky, M. (2005). Creating the discipline of knowledge management: the latest in university research. Boston: Elsevier Butterworth - Heinemann.

Stenmark, D. (2001). Leveraging tacit organisational knowledge. journal of Management Information Systems, 17(3), 9-24.

Stephenson, K., \& Zelen, M. (1989). Rethinking Centrality: Methods and Examples. Social Networks, 11, 1-37.

Swap, W., Leonard, D., Shields, M., \& Abrams, L. (2001). Using Mentoring and Storytelling to Transfer Knowledge in the Workplace. Journal of Management Information Systems 18(1), 95-114.

Szulanski, G. (1996). Exploring internal stickiness: Impediments to the transfer of best practice within the firm Strategic Management Journal, 17, 27-44.

Szulanski, G. (2000). The process of knowledge transfer: A diachronic analysis of stickiness. Organizational Behaviour and Human Decision Processes, 82(1), 9-27.

Tashakkori, A., \& Teddlie, C. (1998). Mixed methodology: Combining qualitative and quantitative approaches. Thousand, CA: Sage. 
Teddlie, C., \& Yu, F. (2007). Mixed Methods Sampling Journal of Mixed Methods Research, 1(1), 77-100.

Todeva, E. (2006). Business networks : strategy and structure London, New York: Routledge.

Todeva, E., \& Knoke, D. (2005). Strategic Alliances and models of collaboration. Management Decision, 43(1), 123-148.

Trauth, E. M., \& Jessup, L. M. (2000). Understanding Computer-Mediated Discussions: Positivist and Interpretive Analyses of Group Support System Use MIS Quarterly, 24(1), 43-79.

Tushman, M. (1977). Special boundary roles in the innovation process. Administrative Science Quarterly, 22(4), 587-605.

Valente, T. W., Coronges, K. A., Stevens, G. B., \& Cousineau, M. R. (2008). Collaboration and competition in a children's health initiative coalition: A network analysis Evaluation and Program Planning, 31(4), 392-402.

van Baalen, P., Bloemhof-Ruwaard, J., \& van Heck, E. (2005). Knowledge Sharing in an Emerging Network of Practice: The Role of a Knowledge Portal. European Management Journal, 23(3), 300-314.

van Wijk, R., Jansen, J. J. P., \& Lyles, M. A. (2008). Inter- and Intra-Organizational Knowledge Transfer: A Meta-Analytic Review and Assessment of its Antecedents and Consequences. Journal of Management Studies, 45(4), 831853.

Villain, S., Leiner, F., Gaffal, F., \& Pisl, G. (2004). TempoBy-DasTelemedizinPortal der bayerischen Universitätsklinika. Munich: Bayerisches Staatsministerium für Wissenschaft, Forschung und Kunst, Klinikum der Universität München, Siemens AG ICN.

Walsham, G. (1995). Interpretive case studies in IS research:nature and method. European Journal of Information Systems, 4, 74-81.

Wasserman, S., \& Faust, K. (1994). Social Network Analysis: Methods and applications. Cambridge, UK: Cambridge University Press.

Watson-Manheim, M. B., \& Belanger, F. (2007). Communication Media Repertoires: Dealing with the Multiplicity of Media Choices. MIS Quarterly, 31(2), 239265.

Watts, D. J., \& Strogatz, S. (1998). Collective dynamics of "small-world" networks. Nature, 393(6684), 440-442. 
Weatherill, G., \& Burton, P. W. (2009). Delineation of shallow seismic source zones using K-means cluster analysis, with application to the Aegean region. Geophysical Journal International, 176(2), 565 - 588.

Weick, K. E. (1995). Sensemaking in Organizations. Thousand Oaks, CA: Sage.

Weiss, A. (2005). The power of collective intelligence. Collective Intelligence, September 2005, 17-23.

Wenger, E. (1998). Communities of practice. Learning, meaning and identity. Oxford, UK: Cambridge University Press.

Wenger, M. S., \& Ferguson, C. (2006). A Learning Ecology Model for Blended Learning from Sun Microsystems. In C. J. Bonk \& C. R. Graham (Eds.), The Handbook of Blended Learning (pp. 76-91). San Francisco: Pfeiffer.

White, D., \& Houseman, M. (2003). The navigability of strong ties: Small worlds, tie strength, and network topology. Complexity, 8(1), 82-86.

Willging, P. A. (2005). Analyzing asynchronous online interactions through social network analysis techniques and visualizations. University of Illinois at Urbana-Champaign.

Yin, R. K. (1993). Applications of case study research. Newbury Park: Sage.

Yin, R. K. (2003). Case Study Research, Design and Methods (3rd ed.). Newbury Park: Sage Publications.

Yoshino, M. Y., \& Rangan, U. S. (1995). Strategic Alliances: An Entrepreneurial Approach to Globalization: Harvard Business School Press.

Zack, M. H. (1999). Developing a Knowledge Strategy. California Management Review, 41(3), 125-145.

Zander, U., \& Kogut, B. (1995). Knowledge and the Speed of the Transfer and Imitation of Organizational Capabilities: An Empirical Test. Organization Science, 6(1), 76-92.

Zanner, R. A. (2006, 11-12 December). Deliberate Inter-organisational Knowledge Transfer: Recognition and Effective Utilisation of Complimentary Social and Technological Channels. Paper presented at the Australian Conference for Knowledge Management and Intelligent Decision Support (ACKMIDS), Melbourne, Australia.

Zheng, X., Chen, D., \& Wu, Z. (2004, 10-13 October). Secure strategy for networked manufacturing system. Paper presented at the IEEE International Conference on Systems, Man and Cybernetics, Den Haag, The Netherlands. 


\section{Appendices}

\section{Appendix 1: Copy of the online survey}

1. Which organisation are you working for?

2. Your gender?
Male
Female

3. What is your job position?

4. How many years have you been working in this or a similar job position?

$\begin{array}{llll}<1 \text { year } 1-3 \text { years } 4-10 \text { years } 11-19 & 20-30 & >30 \text { years } \\ \text { years years } & \end{array}$

5. For your continuous education, which learning methods would you prefer to use after a face-to-face workshop or seminar? Please indicate a maximum of two options.

\begin{tabular}{|ll|}
\hline online discussion forum and chats & online learning \\
\hline e-book & online interaction with experts \\
\hline
\end{tabular}

Other (please specify below)

6. What are the two most important learning method characteristics?

provides current information

allows for topic exploration provides guidance for learning

allows for communication with peers (participation)

Other (please specify below) 
7. How does an informal communication (e.g. give or seek advice) with your colleagues on a marine biology related work topic typically start ?

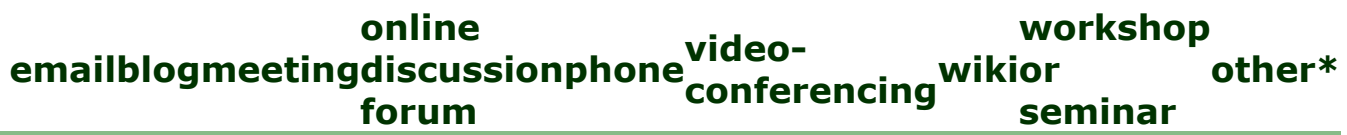

\section{<organisation $1>$} $<$ organisation2> <organisation3> <organisation4> <organisation5> <organisation6>

*Other (please specify below)

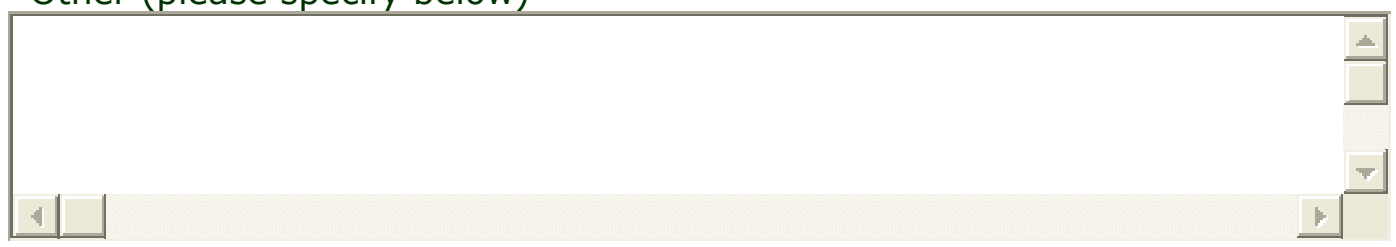

8. How is an informal communication after the initial contact typically followedup? Please indicate a maximum of two options (per organisation)!

$$
\begin{gathered}
\text { online } \\
\text { emailblogmeetingdiscussionphone } \\
\text { forum }
\end{gathered} \text { conferencing }_{\substack{\text { videor } \\
\text { seminar }}}^{\text {workshop }} \text { other* }
$$

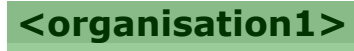

*Other (please specify below) 
9. After the first follow-up, how is an informal contact typically maintained?

Please indicate a maximum of two options (per organisation)!

$\quad \begin{gathered}\text { online } \\ \text { emailblogmeetingdiscussionphone } \\ \text { forum }\end{gathered}$
conferencing
<organisation1>
<organisation2>
<organisation3>
<organisation4>
<organisation5>
<organisation6>

*Other (please specify below)

10. During the last year, how often have you given advice to colleagues working on marine biology related topics?

at least at least at least at least once every once every once every once every never week month 3 months 6 months

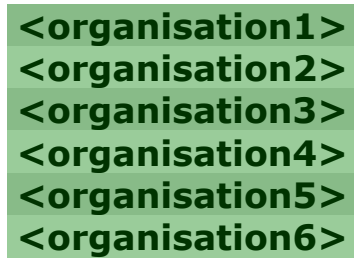


11. Please indicate a maximum of three most important types of content that are communicated when you give advice to colleagues from other organisations.

\section{electronically accessible}

\section{electronically inaccessible (but ${ }^{\text {non-codified }}$ codified)}

\section{an expert's contact details

* non-codified (tacit) content refers to the knowledge that people carry in their minds, but which is not written down (as codified knowledge is). Other (please specify below)

12. What are the two most important media characteristics when you give advice?

\begin{tabular}{|lr|}
\hline provides a trustworthy environment & \multicolumn{2}{c|}{ ability to save information for later } \\
\hline quick transfer of information & reuse \\
\hline ability to use multiple ways of & ease of use \\
\hline transfer & ability to edit information before \\
\hline tctures &
\end{tabular}

Other (please specify below)

13. During the last year, how often have you sought advice from colleagues working on marine biology related topics?
at least at least
at least at least
once every once every once every once every never
week month
3 months 6 months

<organisation1> 
14. Please indicate a maximum of three most important types of content that are communicated when you seek advice from colleagues from other organisations.

\section{electronically accessible}

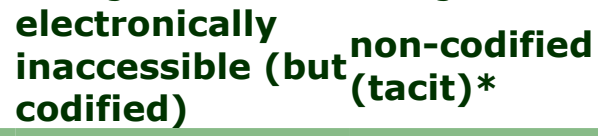
codified)

\section{an expert's contact}

details

a reference to

another source of

information

formal

information(how-to

expertise)

formal

information(facts)

informal information

(how-to expertise)

informal information

(facts)

* non-codified (tacit) content refers to the knowledge that people carry in their minds, but which is not written down (as codified knowledge is). Other (please specify below)

15. What are the two most important media characteristics when you seek advice?

ability to use multiple ways of communicating with text, voice and/or pictures

ability to save information for later transfer reuse

provides a trustworthy environment ease of use ability to edit information before quick transfer of information

Other (please specify below)

Thanks a lot for your participation! 


\section{Appendix 2: Information Sheet}

$<$ Date $>$

Dear <participant>,

\section{Interorganisational knowledge transfer and Information and Communication Technology support among Research and Development organisations}

This letter follows up our conversation in which I expressed my interest in interviewing you on how information and communication technology (ICT) can facilitate knowledge exchange between you and your colleagues from other organisations. Prior to conducting the proposed interview, Victoria University of Wellington requires that I obtain your written informed consent.

\section{Purpose of the Research}

The purpose of this research project is to investigate how knowledge exchange takes place between you and your colleagues from other organisations, such as other research institutes, businesses and governments. It is intended to identify structures and processes of knowledge transfer, to identify purposes and interests of participants to exchange knowledge and to find out how suitable ICT could support these scenarios, for example to provide other collaborative services in addition to workshops/seminars. The research is being undertaken for the purposes of completing the degree of $\mathrm{PhD}$ in Information Systems. A summary of the research findings will be available to you in the form of a business report.

\section{Confidentiality}

All raw data will be kept confidential to myself and my supervisor. Collected, collated and analysed data may be published in case studies, academic journals and presented at conferences. Any information and opinions that you provide will not be attributed to you, and you will not be able to be identified in any way. There will be an opportunity for you to review any written notes or transcripts of recorded sessions that result from the interviews, to ensure that material is recorded accurately. Throughout the project, raw data will be kept under password and/or lock protection and destroyed two years after the conclusion of the project.

Please feel free to contact my supervisor Dr Pak Yoong or myself if you require further information about the project or the informed consent requirement. You may withdraw from this project at any time up until [Date], and in this case, your data will be immediately destroyed and excluded from the study.

The consent form is attached. It includes a request for permission to tape-record interviews. The interviews will last 30-45 minutes. If you agree to participate, please complete the form, sign it and return it to me in the enclosed, stamped and addressed envelope by [Date]. Please contact me if you have any questions. Thank you.

Yours sincerely,

Ms Silke Retzer (PhD student)

clo Associate Professor Pak Yoong, School of Information Management, Victoria University of Wellington, Private Bag, Wellington.

Email: silke.retzer@vuw.ac.n 


\section{Appendix 3: Screenshots of the Wiki tool}

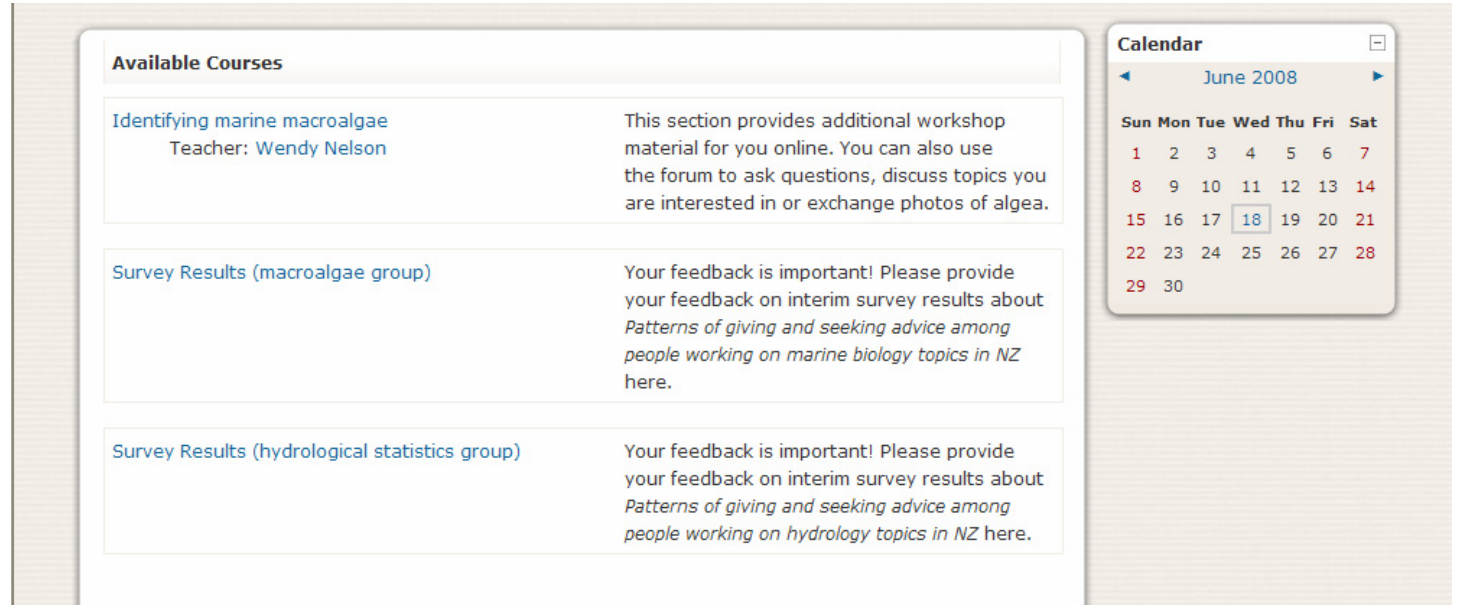

feedback wiki one

View Edit Links History

Reload this page

\section{feedback wiki 1}

1. Why do you think that only a few people working on hydrological topics are using wikis or blogs for seeking and/or giving advice? In this regard, do you think that advice giving/seeking happens more often in groups or between two people? To answer please click the following link: answer page 1/1.

2. Are you participating in any sort of social networking site on the web, for example facebook or Linkedin? If yes, how often and for what purposes are you using it? Would you be interested to use an online social network for exchanging work knowledge with people from other organisations? To answer please click the following link: answer page 1/2. 

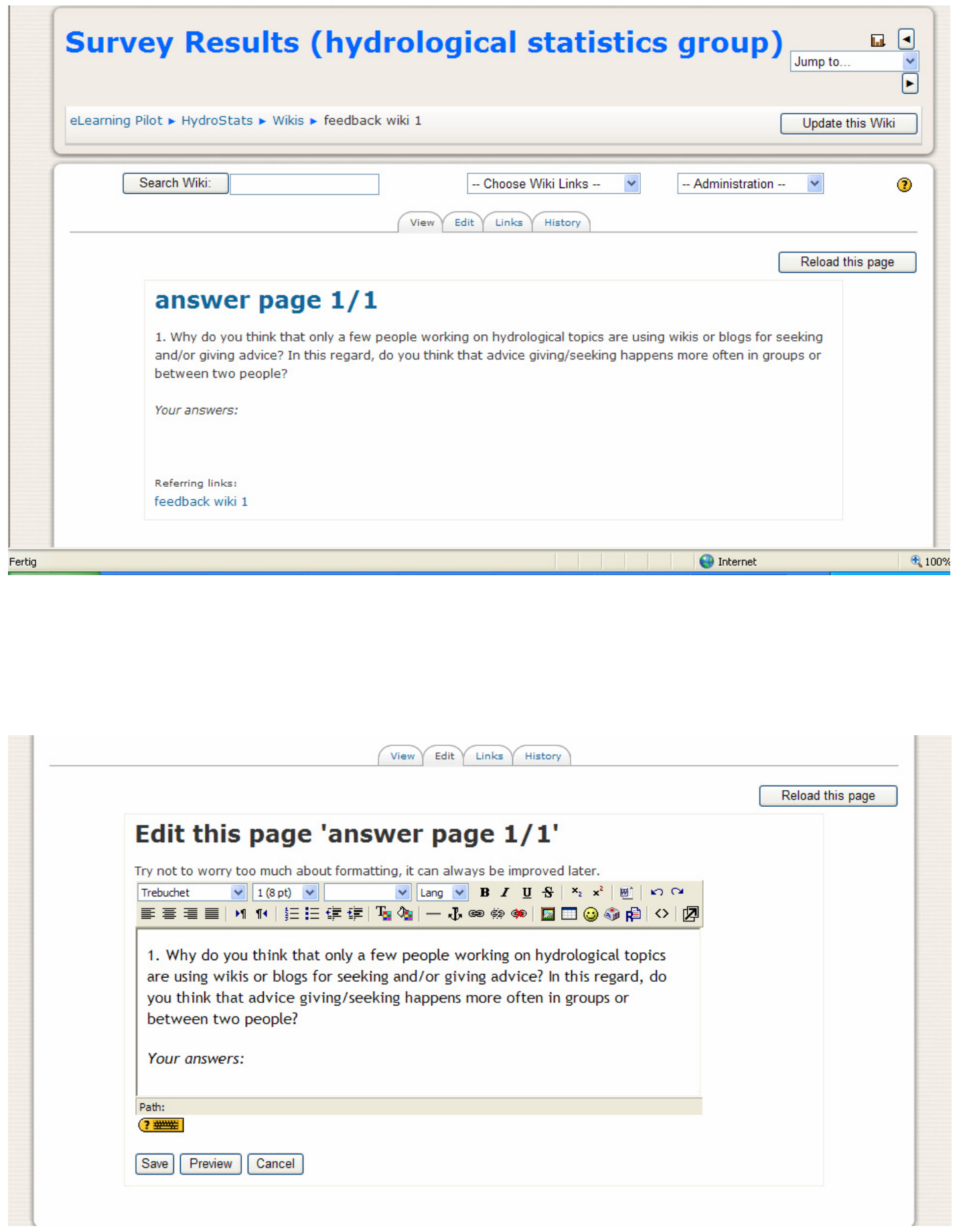


\section{Appendix 4 : Meta-matrix outlining a set of initial categories as a result}

of the cross-case analysis

\begin{tabular}{|c|c|}
\hline Category & Results that all 3 cases had in common \\
\hline Type of network topology & small-world networks \\
\hline $\begin{array}{l}\text { Tie strength: key (strongest) } \\
\text { relationships }\end{array}$ & local governments - private businesses \\
\hline Intermediate relationships & $\begin{array}{l}\text { link a type of orga that is not included in the } \\
\text { most central types of orgas of the small-world } \\
\text { group (linking 'isolates' or weakly tied } \\
\text { organisations) or link two otherwise strongly } \\
\text { connected types ('knowledge brokering tie') }\end{array}$ \\
\hline Levels of interaction & $\begin{array}{l}\text { Key sources and key sinks, knowledge brokers, } \\
\text { balance of interactions between types }\end{array}$ \\
\hline Information centrality & CRIs \\
\hline $\begin{array}{l}\text { Most central groups of organisation } \\
\text { present in small-world network }\end{array}$ & CRIs, private businesses, local governments \\
\hline $\begin{array}{l}\text { Electronically inaccessible types of } \\
\text { knowledge }\end{array}$ & informal factual knowledge \\
\hline $\begin{array}{l}\text { Other most important types of } \\
\text { knowledge }\end{array}$ & $\begin{array}{l}\text { Electronically accessible references to } \\
\text { other sources of knowledge (seek and give) }\end{array}$ \\
\hline $\begin{array}{l}\text { Most important media } \\
\text { characteristics (MST) }\end{array}$ & $\begin{array}{l}\text { ease of use, quick transfer of information, } \\
\text { ability to save information for later reuse }\end{array}$ \\
\hline $\begin{array}{l}\text { Most preferred knowledge services } \\
\text { (Wenger \& Ferguson, 2006) }\end{array}$ & (online) interaction with experts \\
\hline Other ICT use & $\begin{array}{l}\text { email priority, few-non social software, } \\
\text { few discussion forum, video conferencing }\end{array}$ \\
\hline Participant characteristics & $\begin{array}{l}\text { more male participants, but more female } \\
\text { job beginners ( } 0 \text {-3years) }\end{array}$ \\
\hline
\end{tabular}


Appendix 5 : Clustered summary table outlining the clustered categories as key categories of the cross-case analysis.

\begin{tabular}{|l|l|}
\hline \multicolumn{1}{|c|}{ Main category } & Summarised sub-categories \\
\hline Ontological network structure & Small-world network characteristics \\
\hline & $\begin{array}{l}\text { Most central and strongest tied groups of } \\
\text { organisation }\end{array}$ \\
\hline $\begin{array}{l}\text { Types of knowledge } \\
\text { transferred }\end{array}$ & Email advice network (ICT use) \\
\hline & Electronically inaccessible \\
\hline Levels of interaction & Most often transferred \\
\hline Centrality:Type of knowledge - Information \\
centrality
\end{tabular}


Appendix 6: Case 1 survey results - participation by gender

\begin{tabular}{|l|c|c|}
\hline \multicolumn{2}{|l|}{ Your gender? } & \\
\hline Answer & & \\
Options & Response Percent & Response Count \\
\hline Male & $83,8 \%$ & 67 \\
\hline Female & $16,3 \%$ & 13 \\
\hline \multicolumn{2}{|c|}{ answered question } & \\
\hline
\end{tabular}




\section{Appendix 7: Case 1 survey results - types of knowledge transferred}

\section{Types of knowledge transferred for giving advice activities}

\begin{tabular}{|c|c|c|c|}
\hline & electronically accessible & electronically inaccessible & $\begin{array}{l}\text { Response } \\
\text { Count }\end{array}$ \\
\hline $\begin{array}{r}\text { an expert's contact details (know- } \\
\text { who) }\end{array}$ & $88.0 \%(22)$ & $20.0 \%(5)$ & 25 \\
\hline $\begin{array}{r}\text { a reference to another source of } \\
\text { information }\end{array}$ & $100.0 \%(29)$ & $17.2 \%(5)$ & 29 \\
\hline $\begin{array}{r}\text { a formal document(how-to } \\
\text { expertise) }\end{array}$ & $88.9 \%(16)$ & $27.8 \%(5)$ & 18 \\
\hline a formal document(facts) & $88.9 \%(24)$ & $18.5 \%(5)$ & 27 \\
\hline $\begin{array}{r}\text { an informal information (how-to } \\
\text { expertise) }\end{array}$ & $56.7 \%(17)$ & $60.0 \%(18)$ & 30 \\
\hline \multirow[t]{3}{*}{ an informal information (facts) } & $73.9 \%(17)$ & $39.1 \%(9)$ & 23 \\
\hline & & Other (please specify) & 0 \\
\hline & & answered question & 53 \\
\hline
\end{tabular}

\section{Types of knowledge transferred for seeking advice activities}

\begin{tabular}{|c|c|c|c|}
\hline & electronically accessible & electronically inaccessible & $\begin{array}{l}\text { Response } \\
\text { Count }\end{array}$ \\
\hline $\begin{array}{r}\text { an expert's contact details (know- } \\
\text { who) }\end{array}$ & $92.3 \%(24)$ & $19.2 \%(5)$ & 26 \\
\hline $\begin{array}{r}\text { a reference to another source of } \\
\text { information }\end{array}$ & $88.2 \%(30)$ & $20.6 \%(7)$ & 34 \\
\hline $\begin{array}{r}\text { a formal document (how-to } \\
\text { expertise) }\end{array}$ & $95.0 \%(19)$ & $15.0 \%(3)$ & 20 \\
\hline a formal document (facts) & $100.0 \%(25)$ & $8.0 \%(2)$ & 25 \\
\hline $\begin{array}{r}\text { an informal information (how-to } \\
\text { expertise) }\end{array}$ & $65.4 \%(17)$ & $46.2 \%(12)$ & 26 \\
\hline \multirow[t]{3}{*}{ an informal information (facts) } & $72.7 \%(16)$ & $40.9 \%(9)$ & 22 \\
\hline & & Other (please specify) & 1 \\
\hline & & answered question & 53 \\
\hline
\end{tabular}




\section{Appendix 8: Case 1 survey results - contact frequencies}

The dl. file format was used for coding the contact frequency per tie and the number of study participants from each (group of) organisation. In the following dl. file extract the contact frequencies were coded as follows:

\begin{tabular}{|l|l|}
\hline frequency & coding \\
\hline & \\
\hline at least once every week & 4 \\
\hline at least once every month & 3 \\
\hline at least once every 3 months & 2 \\
\hline at least once every 6 months & 1 \\
\hline never & 0 \\
\hline
\end{tabular}

In regard to the contact frequencies and the number of study participants, the following file extract shows the non-aggregated survey data for the advice network. The 'labels' indicate all possible knowledge givers and receivers in this case. These are 27 in this case $(n=27)$. In an ascending order, the listed organisations can be numbered (coded) 1 to 27. The respective code appears in brackets after each organisation, e.g. CRI3 (1). This means CRI3 is coded with a 1 in the following table.

The following numbers represent the coded knowledge givers, the coded knowledge seekers and the coded contact frequencies. The first number in each set of three numbers in the table below indicates the coded knowledge giver whereas the second number indicates the coded knowledge receiver. The third number shows the coded contact frequency. For example, the first set of numbers (1 7 1) indicates CRI3 (1) as the knowledge giver, RegionalCouncill (7) as the knowledge receiver and a contact frequency of at least once every 6 months (1).

\section{dl $\mathbf{n}=\mathbf{2 7}$ format $=$ edgelist1}

labels: CRI3 (1),privatebusiness2 (2), privatebusiness1 (3), RegionalCouncil3 (4) ,privatebusiness3 (5) ,privatebusiness4 (6) ,RegionalCouncill (7), RegionalCouncil4 (8) ,CRI4 (9) ,CRI2 810), privatebusiness5 (11),RegionalCouncil5 (12), privatebusiness6 (13), CRI5 (14), privatebusiness7 (15), centralgovernment (16) ,privatebusiness8 (17), CRI1 (18), privatebusiness9 (19), privatebusiness 10 (20) ,privatebusiness 11 (21), RegionalCouncil6 (22), privatebusiness13 (23) ,privatebusiness 14 (24), University1 (25), University2 (26), RegionalCouncil2 (27) 


\begin{tabular}{|c|c|c|}
\hline data: & 22182 & 1832 \\
\hline 171 & 22272 & 1873 \\
\hline 1101 & 2371 & 1872 \\
\hline 1181 & 23101 & 1872 \\
\hline 273 & 23181 & 1871 \\
\hline 2103 & 2531 & 1871 \\
\hline 373 & 2571 & 1873 \\
\hline 373 & 2631 & 1872 \\
\hline 374 & 26183 & 18102 \\
\hline 374 & 2771 & 18101 \\
\hline 372 & 27103 & 18103 \\
\hline 371 & 27183 & 18101 \\
\hline 3101 & 372 & 18101 \\
\hline 3181 & 3181 & 18272 \\
\hline 3181 & 372 & 18271 \\
\hline 3181 & 372 & 18272 \\
\hline 3181 & 374 & 18272 \\
\hline 3182 & 371 & 18271 \\
\hline 5103 & 3101 & 19182 \\
\hline 5183 & 3182 & 1972 \\
\hline 6101 & 3181 & 2031 \\
\hline 6183 & 473 & 2071 \\
\hline 6272 & 4181 & 20101 \\
\hline 731 & 571 & 20182 \\
\hline 731 & 6181 & 20271 \\
\hline 732 & 6271 & 21181 \\
\hline 7102 & 732 & 2271 \\
\hline 7101 & 7101 & 22102 \\
\hline 7101 & 7181 & 22182 \\
\hline 7101 & 732 & 22273 \\
\hline 7181 & 732 & 23101 \\
\hline 7181 & 732 & 26181 \\
\hline 7181 & 731 & \\
\hline 7182 & 7102 & \\
\hline 871 & 7101 & \\
\hline 8181 & 7101 & \\
\hline
\end{tabular}




\begin{tabular}{|c|c|}
\hline 8181 & 7101 \\
\hline 8183 & 7182 \\
\hline 933 & 7181 \\
\hline 972 & 7181 \\
\hline 9101 & 7271 \\
\hline 9182 & 871 \\
\hline 1072 & 8181 \\
\hline 10182 & 8181 \\
\hline 10183 & 932 \\
\hline 1171 & 972 \\
\hline 11181 & 9101 \\
\hline 1371 & 9182 \\
\hline 13181 & 9271 \\
\hline 13271 & 1073 \\
\hline 1432 & 10183 \\
\hline 1471 & 1173 \\
\hline 1471 & 11183 \\
\hline 14102 & 1272 \\
\hline 14103 & 12101 \\
\hline 14181 & 12181 \\
\hline 14182 & 1332 \\
\hline 14182 & 1373 \\
\hline 1531 & 13181 \\
\hline 1572 & 13273 \\
\hline 15101 & 1432 \\
\hline 15101 & 1472 \\
\hline 15181 & 14103 \\
\hline 15181 & 14183 \\
\hline 1632 & 1471 \\
\hline 1673 & 14102 \\
\hline 16101 & 14181 \\
\hline 16183 & 14182 \\
\hline 17182 & 1531 \\
\hline 1831 & 15181 \\
\hline 1831 & 1531 \\
\hline 1871 & 1573 \\
\hline 1871 & 15102 \\
\hline 1871 & 15182 \\
\hline 1872 & 1632 \\
\hline
\end{tabular}




$\begin{array}{ll}18101 & 1673 \\ 18103 & 16101 \\ 18271 & 16183 \\ 18102 & 17102 \\ 1971 & 17182 \\ 19182 & 1831 \\ 20101 & 1872 \\ 20181 & 18103 \\ 20272 & 18272 \\ 21181 & 1831 \\ 2271 & 1831 \\ 22101 & 1831\end{array}$




\section{Appendix 9: Case 1 survey results - tie strength matrices}

Matrix of valued tie strength among all non-aggregated study participants underlying the visualisation of tie strength with Netdraw:

\begin{tabular}{|c|c|c|c|c|c|c|c|c|c|c|c|c|c|c|c|c|c|c|c|c|c|c|c|c|c|c|c|c|}
\hline & & & 2 & 3 & 4 & 5 & 6 & 7 & 8 & 9 & 10 & & 12 & 13 & 14 & 15 & & 17 & 18 & 19 & 20 & 21 & 22 & 23 & 24 & 25 & & \\
\hline & & $\mathrm{CR}$ & pr & pr & Re & pr & pr & $\operatorname{Re} 1$ & $\operatorname{Re}$ & $\mathrm{CR}$ & $\mathrm{CR}$ & pr & $\operatorname{Re}$ & pr & & pr & ce & $\mathrm{pr}$ & $\mathrm{CR}$ & pr & pr & pr $\mathrm{F}$ & $\operatorname{Re}$ & pr & pr & Un & Un & e \\
\hline 1 & CRI3 & 0 & 0 & 0 & 0 & 0 & 0 & 1 & 0 & 0 & 1 & 0 & 0 & 0 & 0 & 0 & 0 & 0 & 1 & 0 & 0 & 0 & 0 & 0 & 0 & 0 & 0 & \\
\hline 2 & privatebusiness 2 & 0 & 0 & 0 & 0 & 0 & 0 & 3 & 0 & 0 & 3 & 0 & 0 & 0 & 0 & 0 & 0 & 0 & 0 & 0 & 0 & 0 & 0 & 0 & 0 & 0 & 0 & 0 \\
\hline 3 & privatebusiness 1 & 0 & 0 & 0 & 0 & 0 & 0 & 28 & 0 & 0 & 2 & 0 & 0 & 0 & 0 & 0 & 0 & 0 & 10 & 0 & 0 & 0 & 0 & 0 & 0 & 0 & 0 & \\
\hline 4 & RegionalCouncil3 & 0 & 0 & 0 & 0 & 0 & 0 & 3 & 0 & 0 & 0 & 0 & 0 & 0 & 0 & 0 & 0 & 0 & 1 & 0 & 0 & 0 & 0 & 0 & 0 & 0 & 0 & 0 \\
\hline 5 & privatebusiness 3 & 0 & 0 & 0 & 0 & 0 & 0 & 1 & 0 & 0 & 3 & 0 & 0 & 0 & 0 & 0 & 0 & 0 & 3 & 0 & 0 & 0 & 0 & 0 & 0 & 0 & 0 & \\
\hline 6 & privatebusiness 4 & 0 & 0 & 0 & 0 & 0 & 0 & 0 & 0 & 0 & 1 & 0 & 0 & 0 & 0 & 0 & 0 & 0 & 4 & 0 & 0 & 0 & 0 & 0 & 0 & 0 & 0 & \\
\hline 7 & RegionalCouncill & 0 & 0 & 13 & 0 & 0 & 0 & 0 & 0 & 0 & 11 & 0 & 0 & 0 & 0 & 0 & 0 & 0 & 10 & 0 & 0 & 0 & 0 & 0 & 0 & 0 & 0 & \\
\hline 8 & RegionalCouncil4 & 0 & 0 & 0 & 0 & 0 & 0 & 2 & 0 & 0 & 0 & 0 & 0 & 0 & 0 & 0 & 0 & 0 & 7 & 0 & 0 & 0 & 0 & 0 & 0 & 0 & 0 & \\
\hline 9 & CRI 4 & 0 & 0 & 5 & 0 & 0 & 0 & 4 & 0 & 0 & 2 & 0 & 0 & 0 & 0 & 0 & 0 & 0 & 4 & 0 & 0 & 0 & 0 & 0 & 0 & 0 & 0 & \\
\hline 10 & CRI2 & 0 & 0 & 0 & 0 & 0 & 0 & 5 & 0 & 0 & 0 & 0 & 0 & 0 & 0 & 0 & 0 & 0 & 8 & 0 & 0 & 0 & 0 & 0 & 0 & 0 & 0 & \\
\hline 11 & privatebusiness 5 & 0 & 0 & 0 & 0 & 0 & 0 & 4 & 0 & 0 & 0 & 0 & 0 & 0 & 0 & 0 & 0 & 0 & 4 & 0 & 0 & 0 & 0 & 0 & 0 & 0 & 0 & \\
\hline 12 & RegionalCouncil5 & 0 & 0 & 0 & 0 & 0 & 0 & 2 & 0 & 0 & 1 & 0 & 0 & 0 & 0 & 0 & 0 & 0 & 1 & 0 & 0 & 0 & 0 & 0 & 0 & 0 & 0 & \\
\hline 13 & privatebusiness 6 & 0 & 0 & 2 & 0 & 0 & 0 & 4 & 0 & 0 & 0 & 0 & 0 & 0 & 0 & 0 & 0 & 0 & 2 & 0 & 0 & 0 & 0 & 0 & 0 & 0 & 0 & \\
\hline 14 & CRI5 & 0 & 0 & 4 & 0 & 0 & 0 & 5 & 0 & 0 & 10 & 0 & 0 & 0 & 0 & 0 & 0 & 0 & 11 & 0 & 0 & 0 & 0 & 0 & 0 & 0 & 0 & \\
\hline 15 & privatebusiness? & 0 & 0 & 3 & 0 & 0 & 0 & 5 & 0 & 0 & 4 & 0 & 0 & 0 & 0 & 0 & 0 & 0 & 5 & 0 & 0 & 0 & 0 & 0 & 0 & 0 & 0 & \\
\hline 16 & centralgovernment & 0 & 0 & 4 & 0 & 0 & 0 & 6 & 0 & 0 & 2 & 0 & 0 & 0 & 0 & 0 & 0 & 0 & 6 & 0 & 0 & 0 & 0 & 0 & 0 & 0 & 0 & \\
\hline 17 & privatebusiness 8 & 0 & 0 & 0 & 0 & 0 & 0 & 0 & 0 & 0 & 2 & 0 & 0 & 0 & 0 & 0 & 0 & 0 & 4 & 0 & 0 & 0 & 0 & 0 & 0 & 0 & 0 & \\
\hline 18 & CRI1 & 0 & 0 & 8 & 0 & 0 & 0 & 21 & 0 & 0 & 17 & 0 & 0 & 0 & 0 & 0 & 0 & 0 & 0 & 0 & 0 & 0 & 0 & 0 & 0 & 0 & 0 & 1 \\
\hline 19 & privatebusiness 9 & 0 & 0 & 0 & 0 & 0 & 0 & 3 & 0 & 0 & 0 & 0 & 0 & 0 & 0 & 0 & 0 & 0 & 4 & 0 & 0 & 0 & 0 & 0 & 0 & 0 & 0 & \\
\hline 20 & privatebusiness 10 & 0 & 0 & 1 & 0 & 0 & 0 & 1 & 0 & 0 & 2 & 0 & 0 & 0 & 0 & 0 & 0 & 0 & 3 & 0 & 0 & 0 & 0 & 0 & 0 & 0 & 0 & \\
\hline 21 & privatebusiness 11 & 0 & 0 & 0 & 0 & 0 & 0 & 0 & 0 & 0 & 0 & 0 & 0 & 0 & 0 & 0 & 0 & 0 & 2 & 0 & 0 & 0 & 0 & 0 & 0 & 0 & 0 & \\
\hline 22 & RegionalCouncil6 & 0 & 0 & 0 & 0 & 0 & 0 & 2 & 0 & 0 & 3 & 0 & 0 & 0 & 0 & 0 & 0 & 0 & 4 & 0 & 0 & 0 & 0 & 0 & 0 & 0 & 0 & \\
\hline 23 & privatebusiness 13 & 0 & 0 & 0 & 0 & 0 & 0 & 1 & 0 & 0 & 2 & 0 & 0 & 0 & 0 & 0 & 0 & 0 & 1 & 0 & 0 & 0 & 0 & 0 & 0 & 0 & 0 & \\
\hline 24 & privatebusiness 14 & 0 & 0 & 0 & 0 & 0 & 0 & 0 & 0 & 0 & 0 & 0 & 0 & 0 & 0 & 0 & 0 & 0 & 0 & 0 & 0 & 0 & 0 & 0 & 0 & 0 & 0 & \\
\hline 25 & Universit & 0 & 0 & 1 & 0 & 0 & 0 & 1 & 0 & 0 & 0 & 0 & 0 & 0 & 0 & 0 & 0 & 0 & 0 & 0 & 0 & 0 & 0 & 0 & 0 & 0 & 0 & \\
\hline 26 & University & 0 & 0 & 1 & 0 & 0 & 0 & 0 & 0 & 0 & 0 & 0 & 0 & 0 & 0 & 0 & 0 & 0 & 4 & 0 & 0 & 0 & 0 & 0 & 0 & 0 & 0 & \\
\hline & RegionalCouncil2 & 0 & 0 & 0 & 0 & 0 & 0 & 1 & 0 & 0 & 3 & 0 & 0 & 0 & 0 & 0 & 0 & 0 & 3 & 0 & 0 & 0 & 0 & 0 & 0 & 0 & 0 & \\
\hline
\end{tabular}

Matrix of valued tie strength among key organisations underlying the visualisation of tie strength with Netdraw:

\begin{tabular}{|c|c|c|c|c|c|}
\hline & $\begin{array}{r}1 \\
\mathrm{pr}\end{array}$ & ${ }^{2}$ & $\mathrm{CR}$ & $\mathrm{Cr}$ & $\begin{array}{r}5 \\
\text { Fie }\end{array}$ \\
\hline privatebusiness & 0 & 24 & 2 & 10 & \\
\hline 2 FegiønalCaunoili & 13 & 0 & 11 & $\begin{array}{l}10 \\
10\end{array}$ & \\
\hline CRI2 & 0 & 3 & 0 & 4 & \\
\hline CRI 1 & 8 & 17 & 11 & 0 & \\
\hline RegionalCouncil2 & 0 & 1 & 2 & 2 & \\
\hline
\end{tabular}


Matrix of valued tie strength among all groups of organisation underlying the visualisation of tie strength with Netdraw:

\begin{tabular}{|c|c|c|c|c|c|}
\hline & $\mathrm{C}$ & $\begin{array}{r}2 \\
\mathrm{pr}\end{array}$ & $\begin{array}{r}3 \\
10\end{array}$ & & $\begin{array}{r}5 \\
\text { un }\end{array}$ \\
\hline & $\overline{c_{4}}$ & -17 & $-\frac{1}{10}$ & - & - \\
\hline $\begin{array}{r}\text { CFIs } \\
\text { riwate husine:s=s }\end{array}$ & $\begin{array}{l}54 \\
52\end{array}$ & $1 / 3$ & 48 & 0 & \\
\hline local_governments & 42 & 13 & 14 & 0 & \\
\hline central_gavernments & 19 & 7 & 13 & 0 & \\
\hline universities & 4 & 2 & 1 & 0 & [0 \\
\hline
\end{tabular}


Appendix 10: Case 1 univariate statistics

Level of interaction for groups of organisation

By column (key sinks)

\begin{tabular}{|l|c|c|c|c|c|}
\hline & CRIs & $\begin{array}{l}\text { Private } \\
\text { businesses }\end{array}$ & $\begin{array}{l}\text { Local } \\
\text { government }\end{array}$ & $\begin{array}{l}\text { Central } \\
\text { government }\end{array}$ & Universities \\
\hline Mean & 29.25 & 9.75 & 29.25 & 0.0 & 0.0 \\
\hline Std Dev & 18.86 & 5.72 & 22.79 & 0.0 & 0.0 \\
\hline Sum & 117.0 & 39.0 & 117.0 & 0.0 & 0.0 \\
\hline Variance & 355.69 & 32.69 & 519.19 & 0.0 & 0.0 \\
\hline SSQ & 4845.0 & 511.0 & 5499.0 & 0.0 & 0.0 \\
\hline MCSSQ & 1422.75 & 130.75 & 2076.75 & 0.0 & 0.0 \\
\hline Euc Norm & 69.61 & 22.61 & 74.16 & 0.0 & 0.0 \\
\hline Min & 4.0 & 2.0 & 1.0 & 0.0 & 0.0 \\
\hline Max & 52.0 & 17.0 & 55.0 & 0.0 & 0.0 \\
\hline N of Obs & 4.0 & 4.0 & 4.0 & 4.0 & 4.0 \\
\hline
\end{tabular}

By row (key sources)

\begin{tabular}{|l|c|c|c|c|c|}
\hline & CRIs & $\begin{array}{l}\text { Private } \\
\text { businesses }\end{array}$ & $\begin{array}{l}\text { Local } \\
\text { government }\end{array}$ & $\begin{array}{l}\text { Central } \\
\text { government }\end{array}$ & Universities \\
\hline Mean & 16.25 & 26.75 & 13.75 & 9.75 & 1.75 \\
\hline Std Dev & 19.60 & 26.77 & 17.15 & 7.05 & 1.48 \\
\hline Sum & 65.0 & 107.0 & 55.0 & 39.0 & 7.0 \\
\hline Variance & 384.19 & 716.69 & 294.19 & 49.69 & 2.19 \\
\hline SSQ & 2593.0 & 5729.0 & 1933.0 & 579.0 & 21.0 \\
\hline MCSSQ & 1536.75 & 2866.75 & 1176.75 & 198.75 & 8.75 \\
\hline Euc Norm & 50.92 & 75.69 & 43.97 & 24.06 & 4.58 \\
\hline Min & 0.0 & 0.0 & 0.0 & 0.0 & 0.0 \\
\hline Max & 48.0 & 55.0 & 42.0 & 19.0 & 4.0 \\
\hline N of Obs & 4.0 & 4.0 & 4.0 & 4.0 & 4.0 \\
\hline
\end{tabular}


Appendix 11: Case 2 survey results - job experience

\begin{tabular}{|l|c|c|}
\hline \multicolumn{2}{|c|}{ Your gender? } & \multicolumn{2}{|c|}{} \\
\hline \multirow{2}{*}{$\begin{array}{l}\text { Answer } \\
\text { Options }\end{array}$} & Response Percent & Response Count \\
\hline Male & $55,6 \%$ & 45 \\
\hline Female & $44,4 \%$ & 36 \\
\hline \multicolumn{2}{|c|}{ answered question } \\
\hline
\end{tabular}




\section{Appendix 12: Case 2 survey results - types of knowledge transferred}

\section{Types of knowledge transferred for giving advice activities}

11. Please indicate a maximum of three most important types of content that are communicated when you give advice to colleagues from other organisations.

\begin{tabular}{|c|c|c|c|c|}
\hline & electronically accessible & $\begin{array}{c}\text { electronically } \\
\text { inaccessible (but } \\
\text { codified) }\end{array}$ & non-codified (tacit) ${ }^{\star}$ & $\begin{array}{l}\text { Response } \\
\text { Count }\end{array}$ \\
\hline an expert's contact details & $83.9 \%(26)$ & $12.9 \%(4)$ & $12.9 \%(4)$ & 31 \\
\hline $\begin{array}{r}\text { a reference to another source of } \\
\text { information }\end{array}$ & $76.7 \%(33)$ & $27.9 \%(12)$ & $18.6 \%(8)$ & 43 \\
\hline formal information(how-to expertise) & $81.3 \%(26)$ & $12.5 \%(4)$ & $28.1 \%(9)$ & 32 \\
\hline formal information(facts) & $76.9 \%(30)$ & $28.2 \%(11)$ & $23.1 \%(9)$ & 39 \\
\hline $\begin{array}{r}\text { informal information (how-to } \\
\text { expertise) }\end{array}$ & $44.1 \%(15)$ & $11.8 \%(4)$ & $76.5 \%(26)$ & 34 \\
\hline informal information (facts) & $51.7 \%(15)$ & $24.1 \%(7)$ & $62.1 \%(18)$ & 29 \\
\hline \multicolumn{4}{|c|}{$\begin{array}{l}\text { *non-codified (tacit) content refers to the knowledge that people carry in their minds, but which is not written down } \\
\text { (as codified knowledge is). Other (please specify below) }\end{array}$} & 1 \\
\hline & & & inswered que & 63 \\
\hline
\end{tabular}

\section{Types of knowledge transferred for seeking advice activities}

\begin{tabular}{|c|c|c|c|c|}
\hline & electronically accessible & $\begin{array}{c}\text { electronically } \\
\text { inaccessible (but } \\
\text { codified) }\end{array}$ & non-codified (tacit) ${ }^{\star}$ & $\begin{array}{l}\text { Response } \\
\text { Count }\end{array}$ \\
\hline an expert's contact details & $84.8 \%(28)$ & $15.2 \%(5)$ & $21.2 \%(7)$ & 33 \\
\hline $\begin{array}{r}\text { a reference to another source of } \\
\text { information }\end{array}$ & $86.1 \%(31)$ & $19.4 \%(7)$ & $13.9 \%(5)$ & 36 \\
\hline $\begin{array}{r}\text { formal information (how-to } \\
\text { expertise) }\end{array}$ & $62.1 \%(18)$ & $20.7 \%(6)$ & $51.7 \%(15)$ & 29 \\
\hline formal information (facts) & $76.9 \%(30)$ & $25.6 \%(10)$ & $17.9 \%(7)$ & 39 \\
\hline $\begin{array}{r}\text { informal information (how-to } \\
\text { expertise) }\end{array}$ & $52.4 \%(22)$ & $11.9 \%(5)$ & $64.3 \%(27)$ & 42 \\
\hline informal information (facts) & $64.0 \%(16)$ & $20.0 \%(5)$ & $44.0 \%(11)$ & 25 \\
\hline \multicolumn{4}{|c|}{$\begin{array}{r}\text { * non-codified (tacit) content refers to the knowledge that people carry in their minds, but which is not written down (as codified } \\
\text { knowledge is). Other (please specify below) }\end{array}$} & 0 \\
\hline & & & answered question & 63 \\
\hline
\end{tabular}




\section{Appendix 13: Case 2 survey results - tie strength matrices}

Matrix of valued tie strength among all non-aggregated study participants underlying the visualisation of tie strength with Netdraw:

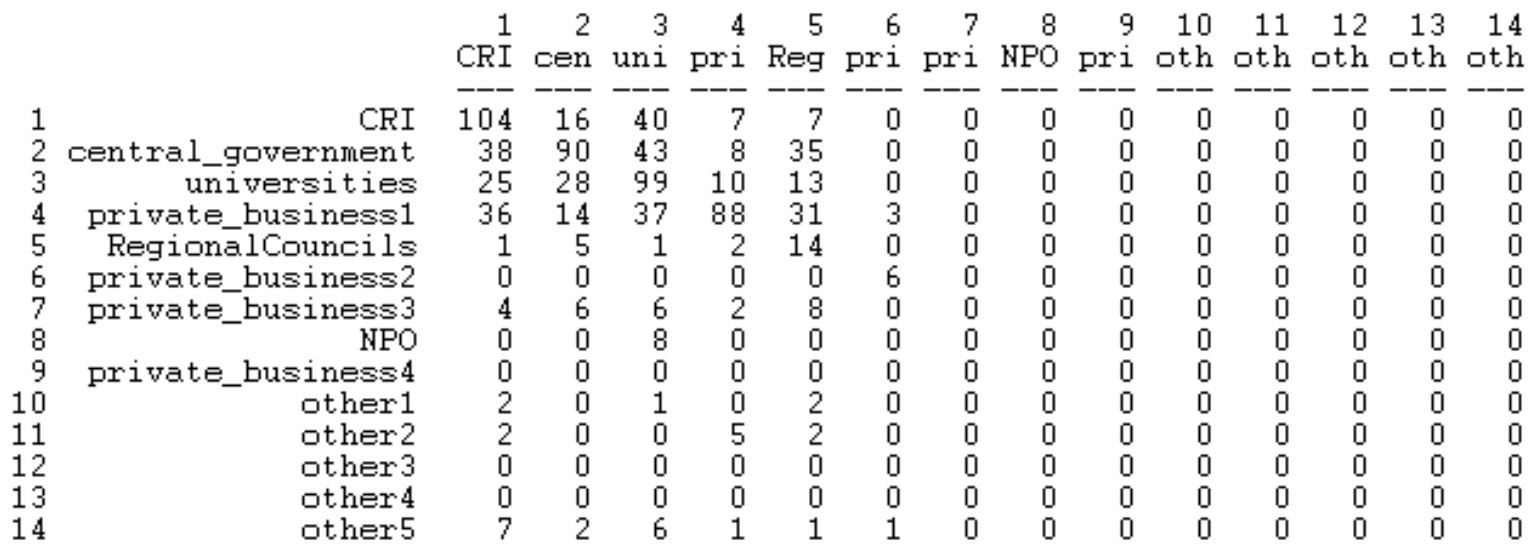

Matrix of valued tie strength among key (groups of) organisations underlying the visualisation of tie strength with Netdraw:

$$
\begin{array}{rrrrrrr} 
& & 1 & 2 & 3 & 4 & 5 \\
& & \text { CRI } & \text { Cen } & \text { Uni } & \text { pri } & \text { Reg } \\
\cline { 3 - 5 } & & 104 & 16 & 40 & 7 & 7 \\
2 & \text { central_government } & 38 & 90 & 43 & 8 & 35 \\
3 & \text { Universities } & 25 & 28 & 99 & 10 & 13 \\
4 & \text { private_business1 } & 36 & 14 & 37 & 88 & 31 \\
5 & \text { RegionalCouncils } & 1 & 5 & 1 & 2 & 14
\end{array}
$$

Matrix of valued tie strength among all groups of organisation underlying the visualisation of tie strength with Netdraw:

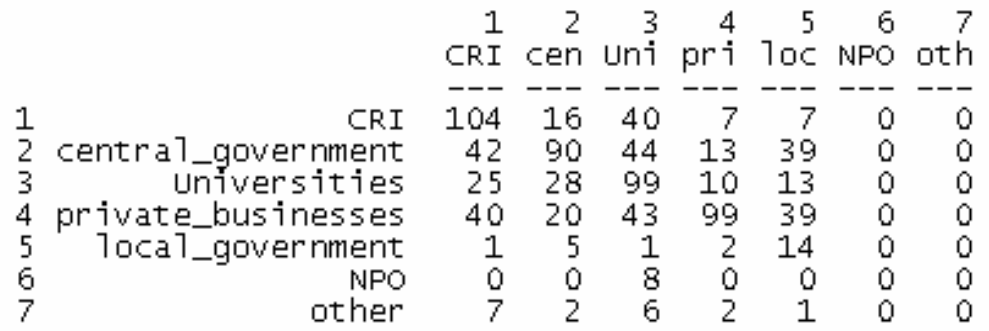




\section{Appendix 14: Case 2 survey results - contact frequencies}

The dl. file format was used for coding the contact frequency per tie and the number of study participants from each (group of) organisation.

In the following $\mathrm{dl}$. file extract the contact frequencies were coded as follows:

\begin{tabular}{|l|l|}
\hline frequency & coding \\
\hline & \\
\hline at least once every week & 4 \\
\hline at least once every month & 3 \\
\hline at least once every 3 months & 2 \\
\hline at least once every 6 months & 1 \\
\hline never & 0 \\
\hline
\end{tabular}

In regard to the contact frequencies and the number of study participants, the following file extract shows the non-aggregated survey data for the advice network. The 'labels' indicate all possible knowledge givers and receivers in this case. These are 14 in this case $(n=14)$. In an ascending order, they are numbered (coded) 1 to 14 . The respective code appears in brackets after each (group of) organisation, e.g. CRIs (1). This means CRIs are coded with a 1 in the following table.

The following numbers represent the coded knowledge givers, the coded knowledge seekers and the coded contact frequencies. The first number in each set of three numbers in the table below indicates the coded knowledge giver whereas the second number indicates the coded knowledge receiver. The third number shows the coded contact frequency. For example, the first set of numbers $\left(\begin{array}{lll}1 & 1 & 3\end{array}\right)$ indicates a knowledge exchange within CRIs (1) with a relatively high contact frequency of at least once every month (3).

dl $\mathbf{n}=14$ format $=$ edgelist 1

labels: CRIs (1), central_government (2), universities (3),private_business1 (4) ,RegionalCouncils (5), private_business2 (6), private_business3 (7) ,NPO (8) ,private_business4 (9), other1 (10), other2 (11), other3 (12), other4 (13), other5 (14)

$\begin{array}{lll}\text { data: } & 150 & 420 \\ 113 & 160 & 450 \\ 133 & 114 & 444 \\ 122 & 310 & 212 \\ 333 & 333 & 231 \\ 114 & 320 & 224 \\ 113 & 350 & 251\end{array}$




\begin{tabular}{|c|c|c|}
\hline 332 & 1111 & 240 \\
\hline 210 & 1130 & 260 \\
\hline 231 & 1151 & 310 \\
\hline 224 & 1142 & 320 \\
\hline 252 & 710 & 330 \\
\hline 240 & 720 & 340 \\
\hline 260 & 730 & 350 \\
\hline 212 & 740 & 360 \\
\hline 231 & 750 & 112 \\
\hline 224 & 760 & 133 \\
\hline 252 & 311 & 114 \\
\hline 241 & 334 & 132 \\
\hline 260 & 320 & 122 \\
\hline 411 & 340 & 151 \\
\hline 430 & 350 & 142 \\
\hline 420 & 360 & 433 \\
\hline 450 & 333 & 443 \\
\hline 444 & 310 & 211 \\
\hline 211 & 334 & 231 \\
\hline 231 & 321 & 223 \\
\hline 223 & 351 & 252 \\
\hline 252 & 340 & 240 \\
\hline 240 & 360 & 260 \\
\hline 260 & 312 & 213 \\
\hline 413 & 334 & 233 \\
\hline 432 & 321 & 224 \\
\hline 421 & 340 & 251 \\
\hline 453 & 350 & 240 \\
\hline 444 & 360 & 260 \\
\hline 461 & 311 & 510 \\
\hline 411 & 330 & 530 \\
\hline 432 & 321 & 522 \\
\hline 421 & 351 & 551 \\
\hline 452 & 341 & 540 \\
\hline 444 & 310 & 560 \\
\hline 460 & 334 & 211 \\
\hline 410 & 320 & 232 \\
\hline 452 & 340 & 223 \\
\hline 443 & 350 & 240 \\
\hline
\end{tabular}




\begin{tabular}{|c|c|c|}
\hline 211 & 360 & 250 \\
\hline 232 & 1010 & 260 \\
\hline 224 & 1031 & 443 \\
\hline 251 & 1020 & 213 \\
\hline 240 & 1052 & 233 \\
\hline 260 & 1040 & 224 \\
\hline 313 & 1060 & 251 \\
\hline 334 & 313 & 240 \\
\hline 321 & 333 & 260 \\
\hline 350 & 324 & 412 \\
\hline 341 & 352 & 431 \\
\hline 360 & 341 & 421 \\
\hline 413 & 360 & 453 \\
\hline 432 & 112 & 444 \\
\hline 421 & 510 & 460 \\
\hline 453 & 531 & 1413 \\
\hline 444 & 521 & 1433 \\
\hline 460 & 552 & 1420 \\
\hline 213 & 541 & 1450 \\
\hline 234 & 560 & 1460 \\
\hline 224 & 834 & 1441 \\
\hline 252 & 332 & 333 \\
\hline 241 & 112 & 321 \\
\hline 260 & 120 & 351 \\
\hline 412 & 130 & 342 \\
\hline 434 & 140 & 332 \\
\hline 420 & 150 & 360 \\
\hline 450 & 160 & 113 \\
\hline 444 & 711 & 133 \\
\hline 460 & 733 & 554 \\
\hline 410 & 722 & 334 \\
\hline 420 & 754 & 323 \\
\hline 430 & 721 & 310 \\
\hline 440 & 760 & 340 \\
\hline 450 & 330 & 350 \\
\hline 460 & 113 & 360 \\
\hline 113 & 131 & 113 \\
\hline 131 & 121 & 113 \\
\hline 121 & 140 & 133 \\
\hline
\end{tabular}




\begin{tabular}{|c|c|c|}
\hline 140 & 150 & 120 \\
\hline 150 & 160 & 140 \\
\hline 160 & 114 & 150 \\
\hline 210 & 131 & 160 \\
\hline 231 & 121 & 114 \\
\hline 224 & 140 & 131 \\
\hline 252 & 150 & 120 \\
\hline 240 & 160 & 151 \\
\hline 260 & 663 & 140 \\
\hline 442 & 114 & 160 \\
\hline 334 & 130 & 113 \\
\hline 212 & 121 & 312 \\
\hline 233 & 150 & 334 \\
\hline 224 & 141 & 322 \\
\hline 241 & 160 & 350 \\
\hline 251 & 333 & 1111 \\
\hline 260 & 114 & 1151 \\
\hline 412 & 114 & 1143 \\
\hline 432 & 333 & 712 \\
\hline 423 & 213 & 732 \\
\hline 452 & 231 & 722 \\
\hline 444 & 224 & 752 \\
\hline 460 & 251 & 741 \\
\hline 413 & 240 & 760 \\
\hline 431 & 260 & 311 \\
\hline 420 & 212 & 334 \\
\hline 450 & 231 & 320 \\
\hline 444 & 224 & 351 \\
\hline 212 & 252 & 341 \\
\hline 231 & 241 & 360 \\
\hline 224 & 260 & 312 \\
\hline 241 & 412 & 334 \\
\hline 251 & 432 & 320 \\
\hline 260 & 421 & 340 \\
\hline 310 & 452 & 350 \\
\hline 320 & 442 & 360 \\
\hline 330 & 212 & 310 \\
\hline 340 & 231 & 334 \\
\hline 350 & 223 & 322 \\
\hline
\end{tabular}




\begin{tabular}{|c|c|c|}
\hline 360 & 252 & 352 \\
\hline 112 & 240 & 340 \\
\hline 131 & 260 & 360 \\
\hline 114 & 413 & 312 \\
\hline 134 & 433 & 332 \\
\hline 123 & 421 & 321 \\
\hline 142 & 453 & 351 \\
\hline 152 & 444 & 340 \\
\hline 410 & 461 & 360 \\
\hline 433 & 411 & 311 \\
\hline 443 & 431 & 320 \\
\hline 211 & 420 & 330 \\
\hline 231 & 452 & 340 \\
\hline 224 & 444 & 350 \\
\hline 252 & 460 & 360 \\
\hline 240 & 452 & 334 \\
\hline 260 & 443 & 310 \\
\hline 211 & 211 & 320 \\
\hline 233 & 231 & 340 \\
\hline 224 & 224 & 350 \\
\hline 252 & 251 & 360 \\
\hline 240 & 240 & 1012 \\
\hline 260 & 260 & 313 \\
\hline 510 & 314 & 333 \\
\hline 530 & 334 & 324 \\
\hline 521 & 322 & 353 \\
\hline 551 & 350 & 340 \\
\hline 540 & 342 & 360 \\
\hline 211 & 360 & 112 \\
\hline 231 & 413 & 511 \\
\hline 222 & 432 & 530 \\
\hline 240 & 421 & 521 \\
\hline 250 & 452 & 552 \\
\hline 260 & 444 & 541 \\
\hline 444 & 460 & 560 \\
\hline 211 & 213 & 834 \\
\hline 232 & 234 & 332 \\
\hline 224 & 224 & 114 \\
\hline 251 & 252 & 133 \\
\hline
\end{tabular}




\begin{tabular}{|c|c|c|}
\hline 240 & 241 & 122 \\
\hline 260 & 260 & 140 \\
\hline 412 & 413 & 150 \\
\hline 431 & 433 & 160 \\
\hline 422 & 420 & 711 \\
\hline 454 & 450 & 731 \\
\hline 444 & 444 & 721 \\
\hline 1414 & 460 & 752 \\
\hline 1433 & 411 & 741 \\
\hline 1422 & 431 & 760 \\
\hline 1451 & 444 & 114 \\
\hline 1440 & 114 & 133 \\
\hline 1461 & 132 & 121 \\
\hline 333 & 121 & 151 \\
\hline 322 & 150 & 140 \\
\hline 351 & 160 & 160 \\
\hline 342 & 141 & 114 \\
\hline 333 & 211 & 131 \\
\hline 114 & 231 & 120 \\
\hline 133 & 224 & 140 \\
\hline 152 & 253 & 150 \\
\hline 554 & 241 & 160 \\
\hline 310 & 260 & 663 \\
\hline 333 & 441 & 114 \\
\hline 323 & 334 & 131 \\
\hline 340 & 211 & 120 \\
\hline 350 & 233 & 150 \\
\hline 360 & 224 & 141 \\
\hline 113 & 251 & 160 \\
\hline 113 & 241 & \\
\hline 132 & 260 & \\
\hline 120 & 412 & \\
\hline 140 & 433 & \\
\hline 150 & 422 & \\
\hline 160 & 451 & \\
\hline 114 & 444 & \\
\hline 132 & 461 & \\
\hline 121 & 412 & \\
\hline 140 & 431 & \\
\hline
\end{tabular}


Appendix 15: Case 3 survey results - job experience

\begin{tabular}{|l|c|c|}
\hline \multicolumn{2}{|l|}{ Your gender? } & \\
\hline Answer & Response Percent & Response Count \\
Options & $53,8 \%$ & 28 \\
\hline Male & $46,2 \%$ & 24 \\
\hline Female & answered question & \\
\hline \multicolumn{2}{|c|}{} \\
\hline
\end{tabular}




\section{Appendix 16: Case 3 survey results - types of knowledge transferred}

\section{Types of knowledge transferred for giving advice activities in strong ties}

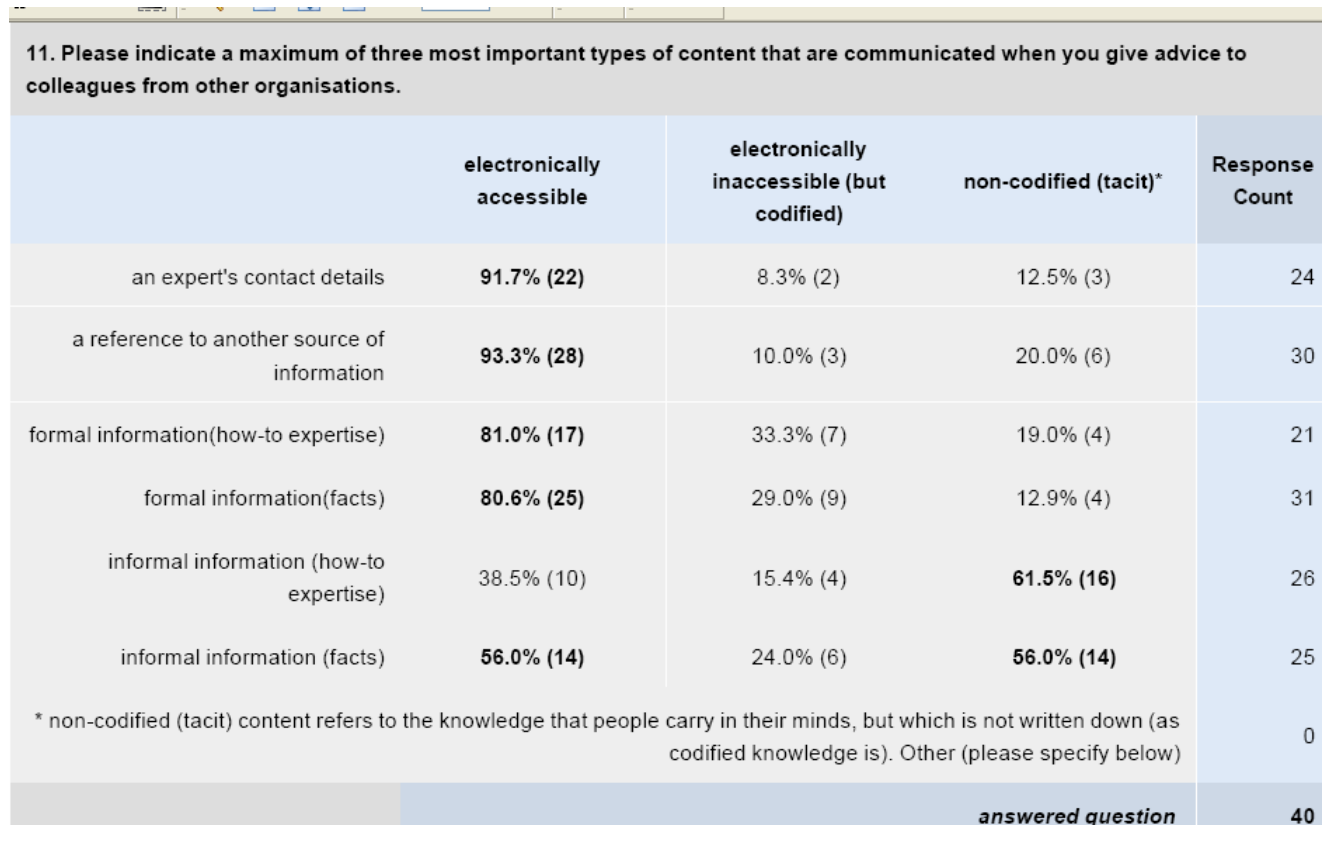

\section{Types of knowledge transferred for seeking advice activities in strong ties}

\begin{tabular}{|c|c|c|c|c|}
\hline & $\begin{array}{l}\text { electronically } \\
\text { accessible }\end{array}$ & $\begin{array}{c}\text { electronically } \\
\text { inaccessible (but } \\
\text { codified) }\end{array}$ & non-codified (tacit) ${ }^{*}$ & $\begin{array}{l}\text { Response } \\
\text { Count }\end{array}$ \\
\hline an expert's contact details & $95.2 \%(20)$ & $4.8 \%(1)$ & $14.3 \%(3)$ & 21 \\
\hline $\begin{array}{r}\text { a reference to another source of } \\
\text { information }\end{array}$ & $90.3 \%(28)$ & $9.7 \%(3)$ & $16.1 \%(5)$ & 31 \\
\hline $\begin{array}{r}\text { formal information (how-to } \\
\text { expertise) }\end{array}$ & $84.2 \%(16)$ & $10.5 \%(2)$ & $15.8 \%(3)$ & 19 \\
\hline formal information (facts) & $61.3 \%(19)$ & $25.8 \%(8)$ & $38.7 \%(12)$ & 31 \\
\hline $\begin{array}{r}\text { informal information (how-to } \\
\text { expertise) }\end{array}$ & $52.9 \%(9)$ & $17.6 \%(3)$ & $58.8 \%(10)$ & 17 \\
\hline informal information (facts) & $38.1 \%(8)$ & $23.8 \%(5)$ & $66.7 \%(14)$ & 21 \\
\hline \multicolumn{4}{|c|}{$\begin{array}{r}\text { * non-codified (tacit) content refers to the knowledge that people carry in their minds, but which is not written down (as } \\
\text { codified knowledge is). Other (please specify below) }\end{array}$} & 1 \\
\hline & & & answered question & 38 \\
\hline
\end{tabular}




\section{Types of knowledge transferred for giving advice activities in weak ties}

\begin{tabular}{|c|c|c|c|c|}
\hline & $\begin{array}{l}\text { electronically } \\
\text { accessible }\end{array}$ & $\begin{array}{c}\text { electronically } \\
\text { inaccessible (but } \\
\text { codified) }\end{array}$ & non-codified (tacit) ${ }^{*}$ & $\begin{array}{l}\text { Response } \\
\text { Count }\end{array}$ \\
\hline an expert's contact details & $60.0 \%(3)$ & $60.0 \%(3)$ & $80.0 \%(4)$ & 5 \\
\hline $\begin{array}{r}\text { a reference to another source of } \\
\text { information }\end{array}$ & $66.7 \%(4)$ & $66.7 \%(4)$ & $66.7 \%(4)$ & 6 \\
\hline formal information(how-to expertise) & $75.0 \%(3)$ & $50.0 \%(2)$ & $50.0 \%(2)$ & 4 \\
\hline formal information(facts) & $80.0 \%(4)$ & $60.0 \%(3)$ & $40.0 \%(2)$ & 5 \\
\hline $\begin{array}{r}\text { informal information (how-to } \\
\text { expertise) }\end{array}$ & $80.0 \%(4)$ & $20.0 \%(1)$ & $60.0 \%(3)$ & 5 \\
\hline informal information (facts) & $50.0 \%(3)$ & $50.0 \%(3)$ & $66.7 \%(4)$ & 6 \\
\hline \multicolumn{4}{|c|}{$\begin{array}{r}\text { * non-codified (tacit) content refers to the knowledge that people carry in their minds, but which is not written down (as } \\
\text { codified knowledge is). Other (please specify below) }\end{array}$} & 1 \\
\hline & & & ered qu & 6 \\
\hline
\end{tabular}

\section{Types of knowledge transferred for seeking advice activities in weak ties}

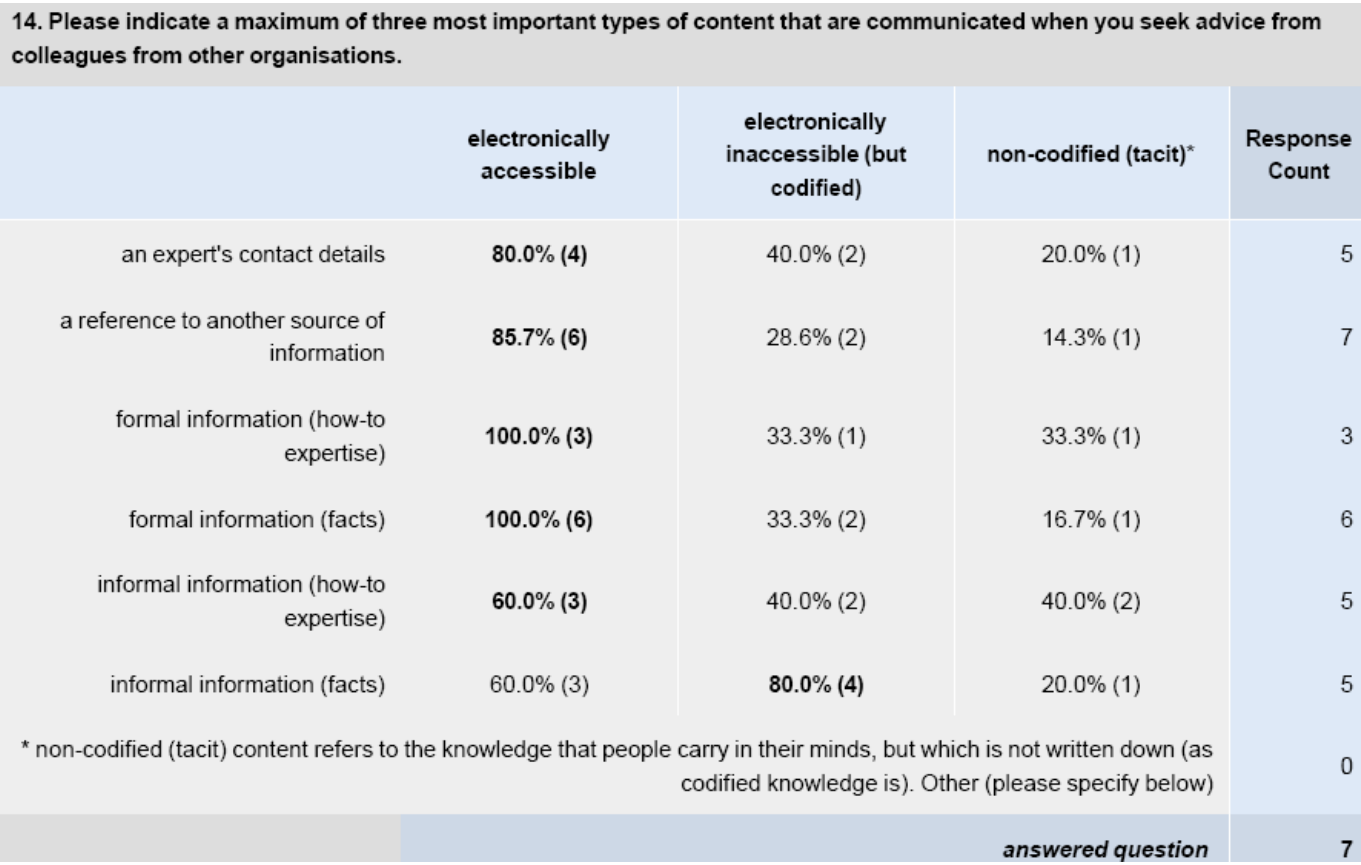




\section{Appendix 17: Case 3 survey results - tie strength matrices}

Matrix of valued tie strength among all groups of organisation underlying the visualisation of tie strength with Netdraw:

\begin{tabular}{rrrrrrrr} 
& & 1 & 2 & 3 & 4 & 5 & 6 \\
& & CR & un & pr & ce & lo & ot \\
\cline { 3 - 6 } & -1 & -- & -- & -- & -- & -- \\
1 & 49 & 22 & 19 & 23 & 30 & 0 \\
2 & universities & 6 & 26 & 8 & 13 & 14 & 0 \\
3 & private_businesses & 17 & 16 & 31 & 29 & 25 & 0 \\
4 & Central_government & 9 & 6 & 14 & 48 & 25 & 0 \\
5 & local_government & 26 & 20 & 35 & 36 & 42 & 0 \\
6 & others & 1 & 1 & 0 & 0 & 2 & 0
\end{tabular}




\section{Appendix 18: Case 3 survey results - contact frequencies}

The dl. file format was used for coding the contact frequency per tie and the number of study participants from each (group of) organisation.

In the following $\mathrm{dl}$. file extract the contact frequencies were coded as follows:

\begin{tabular}{|l|l|}
\hline frequency & coding \\
\hline & \\
\hline at least once every week & 4 \\
\hline at least once every month & 3 \\
\hline at least once every 3 months & 2 \\
\hline at least once every 6 months & 1 \\
\hline never & 0 \\
\hline
\end{tabular}

In regard to the contact frequencies and the number of study participants, the following file extract shows the non-aggregated survey data for the advice network. The 'labels' indicate all possible knowledge givers and receivers in this case. These are 6 in this case $(n=6)$. In an ascending order, they can be numbered (coded) 1 to 6 . The respective code appears in brackets after each group of organisation, e.g. CRIs (1). This means CRIs are coded with a 1 in the following table.

The following numbers represent the coded knowledge givers, the coded knowledge seekers and the coded contact frequencies. The first number in each set of three numbers in the table below indicates the coded knowledge giver whereas the second number indicates the coded knowledge receiver. The third number shows the coded contact frequency. For example, the first set of numbers $\left(\begin{array}{lll}4 & 3 & 3\end{array}\right)$ indicates central government (4) as the knowledge giver, private businesses (3) as the knowledge receivers and a relatively high contact frequency of at least once every month (3).

dl $n=6$ format $=$ edgelist 1

labels: CRIs (1), universities (2),private_businesses (3), central_government (4) ,local_government (5), others (6)

$\begin{array}{lll}\text { data: } & 333 & 140 \\ 433 & 342 & 140 \\ 442 & 352 & 150 \\ 452 & 512 & 512 \\ 511 & 521 & 521 \\ 521 & 533 & 531 \\ 532 & 541 & 543 \\ 543 & 542 & 541\end{array}$




\begin{tabular}{|c|c|c|}
\hline 553 & 553 & 552 \\
\hline 512 & 312 & 510 \\
\hline 523 & 320 & 520 \\
\hline 534 & 333 & 531 \\
\hline 541 & 340 & 540 \\
\hline 541 & 341 & 540 \\
\hline 554 & 353 & 551 \\
\hline 511 & 311 & 113 \\
\hline 520 & 324 & 120 \\
\hline 531 & 333 & 130 \\
\hline 542 & 340 & 143 \\
\hline 540 & 344 & 142 \\
\hline 552 & 352 & 152 \\
\hline 411 & 313 & 111 \\
\hline 421 & 321 & 151 \\
\hline 431 & 332 & 120 \\
\hline 440 & 340 & 130 \\
\hline 442 & 340 & 140 \\
\hline 451 & 351 & 140 \\
\hline 211 & 410 & 221 \\
\hline 224 & 420 & 310 \\
\hline 233 & 432 & 320 \\
\hline 241 & 440 & 331 \\
\hline 241 & 443 & 342 \\
\hline 253 & 452 & 341 \\
\hline 221 & 311 & 353 \\
\hline 411 & 321 & 552 \\
\hline 420 & 333 & 413 \\
\hline 433 & 340 & 420 \\
\hline 441 & 343 & 430 \\
\hline 444 & 353 & 443 \\
\hline 453 & 211 & 440 \\
\hline 442 & 224 & 450 \\
\hline 442 & 231 & 411 \\
\hline 211 & 240 & 420 \\
\hline 223 & 241 & 443 \\
\hline 231 & 252 & 441 \\
\hline 240 & 511 & 451 \\
\hline 240 & 520 & 511 \\
\hline
\end{tabular}




\begin{tabular}{|c|c|c|}
\hline 251 & 531 & 520 \\
\hline 511 & 540 & 530 \\
\hline 522 & 541 & 540 \\
\hline 533 & 552 & 541 \\
\hline 540 & 311 & 550 \\
\hline 541 & 310 & 113 \\
\hline 554 & 333 & 123 \\
\hline 550 & 341 & 130 \\
\hline 114 & 341 & 140 \\
\hline 123 & 352 & 140 \\
\hline 134 & 652 & 150 \\
\hline 144 & 610 & 333 \\
\hline 141 & 620 & 111 \\
\hline 154 & 630 & 121 \\
\hline 110 & 640 & 113 \\
\hline 120 & 640 & 121 \\
\hline 130 & 223 & 133 \\
\hline 140 & 242 & 142 \\
\hline 140 & 251 & 141 \\
\hline 151 & 520 & 152 \\
\hline 511 & 531 & 114 \\
\hline 520 & 541 & 122 \\
\hline 531 & 553 & 132 \\
\hline 542 & 112 & 140 \\
\hline 540 & 114 & 140 \\
\hline 551 & 122 & 153 \\
\hline 510 & 131 & 551 \\
\hline 520 & 140 & 511 \\
\hline 533 & 140 & 521 \\
\hline 540 & 152 & 531 \\
\hline 540 & 411 & 541 \\
\hline 551 & 420 & 550 \\
\hline 112 & 431 & 322 \\
\hline 120 & 440 & 332 \\
\hline 132 & 442 & 342 \\
\hline 141 & 452 & 352 \\
\hline 141 & 510 & 512 \\
\hline 152 & 522 & 521 \\
\hline 111 & 532 & 531 \\
\hline
\end{tabular}




\begin{tabular}{|c|c|c|}
\hline 121 & 540 & 541 \\
\hline 131 & 541 & 540 \\
\hline 141 & 550 & 551 \\
\hline 141 & 410 & 312 \\
\hline 152 & 421 & 320 \\
\hline 220 & 430 & 330 \\
\hline 310 & 440 & 340 \\
\hline 321 & 441 & 340 \\
\hline 332 & 451 & 350 \\
\hline 343 & 510 & 314 \\
\hline 342 & 520 & 323 \\
\hline 353 & 530 & 332 \\
\hline 552 & 540 & 343 \\
\hline 411 & 540 & 342 \\
\hline 421 & 550 & 351 \\
\hline 430 & 512 & 311 \\
\hline 444 & 523 & 321 \\
\hline 442 & 533 & 330 \\
\hline 452 & 540 & 341 \\
\hline 410 & 542 & 340 \\
\hline 420 & 553 & 352 \\
\hline 430 & 512 & 410 \\
\hline 443 & 520 & 421 \\
\hline 440 & 530 & 431 \\
\hline 451 & 542 & 440 \\
\hline 510 & 541 & 443 \\
\hline 520 & 553 & 452 \\
\hline 532 & 411 & 311 \\
\hline 541 & 421 & 321 \\
\hline 542 & 431 & 322 \\
\hline 550 & 440 & 340 \\
\hline 114 & 442 & 341 \\
\hline 124 & 451 & 351 \\
\hline 132 & 211 & 222 \\
\hline 141 & 222 & 230 \\
\hline 140 & 231 & 240 \\
\hline 153 & 240 & 241 \\
\hline 333 & 242 & 251 \\
\hline 112 & 252 & 511 \\
\hline
\end{tabular}




\begin{tabular}{|c|c|c|}
\hline 113 & 221 & 520 \\
\hline 121 & 410 & 530 \\
\hline 133 & 421 & 540 \\
\hline 141 & 432 & 540 \\
\hline 141 & 441 & 551 \\
\hline 152 & 443 & 311 \\
\hline 113 & 454 & 320 \\
\hline 122 & 410 & 331 \\
\hline 153 & 420 & 340 \\
\hline 550 & 430 & 340 \\
\hline 512 & 441 & 350 \\
\hline 522 & 441 & 611 \\
\hline 532 & 451 & 621 \\
\hline 541 & 212 & 630 \\
\hline \multirow{25}{*}{550} & 222 & 640 \\
\hline & 232 & 640 \\
\hline & 241 & 650 \\
\hline & 241 & 210 \\
\hline & 252 & 223 \\
\hline & 511 & 230 \\
\hline & 521 & 242 \\
\hline & 530 & 241 \\
\hline & 540 & 252 \\
\hline & 540 & 511 \\
\hline & 551 & 520 \\
\hline & 550 & 532 \\
\hline & 114 & 540 \\
\hline & 121 & 541 \\
\hline & 131 & 552 \\
\hline & 143 & 111 \\
\hline & 140 & 113 \\
\hline & 152 & 121 \\
\hline & 111 & 130 \\
\hline & 120 & 140 \\
\hline & 130 & 140 \\
\hline & & 151 \\
\hline & & 410 \\
\hline & & 420 \\
\hline & & 430 \\
\hline
\end{tabular}


440

442

452

512

522

531

541

542

550 
Appendix 19: cross-case analysis - dichotomised network data per case

Case 1: Dichotomised advice network used for the clustering coefficient and density measure.

\begin{tabular}{|c|c|}
\hline $\begin{array}{rr}1 & \text { CRIs } \\
2 & \text { private_businesses } \\
3 & \text { lacal_governments } \\
4 & \text { central_govermilnts } \\
5 & \text { universities }\end{array}$ & $\begin{array}{lllll}1 & 2 & 3 & 4 & 5 \\
C & p & 1 & 0 & 1 \\
- & - & - & - & -1 \\
1 & 1 & 1 & 0 & 0 \\
1 & 1 & 1 & 0 & 0 \\
1 & 1 & 1 & 0 & 0 \\
1 & 1 & 1 & 0 & 0 \\
1 & 1 & 1 & 0 & 0\end{array}$ \\
\hline
\end{tabular}

Case 1: Comparable random network used for the clustering coefficient measure

\begin{tabular}{|llllll|}
\hline & 1 & 2 & 3 & 4 & 5 \\
& - & - & - & - & - \\
1 & 0 & 1 & 1 & 0 & 1 \\
2 & 0 & 1 & 0 & 1 & 0 \\
3 & 0 & 0 & 1 & 1 & 1 \\
4 & 0 & 1 & 1 & 0 & 1 \\
5 & 0 & 0 & 0 & 1 & 0 \\
\hline
\end{tabular}


Case 2: Dichotomised advice network used for the clustering coefficient and density measure.

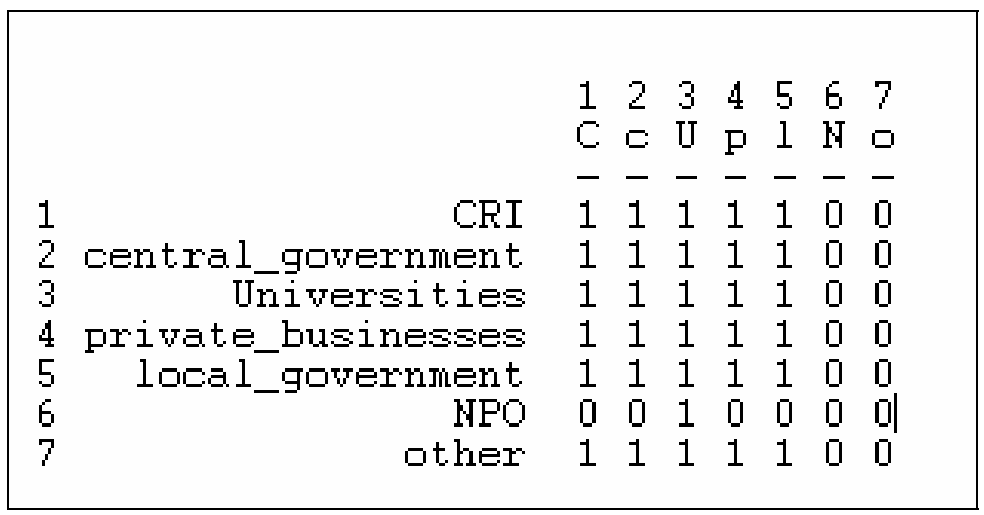

Case 2: comparable random network used for the clustering coefficient measure

\begin{tabular}{|llllllll|}
\hline & 1 & 2 & 3 & 4 & 5 & 6 & 7 \\
& - & - & - & - & - & - & - \\
1 & 0 & 1 & 0 & 1 & 0 & 1 & 0 \\
2 & 0 & 1 & 0 & 0 & 0 & 0 & 1 \\
3 & 1 & 1 & 1 & 0 & 1 & 1 & 0 \\
4 & 0 & 0 & 1 & 0 & 0 & 1 & 0 \\
5 & 1 & 0 & 0 & 0 & 1 & 1 & 1 \\
6 & 1 & 0 & 0 & 0 & 0 & 0 & 0 \\
7 & 0 & 1 & 1 & 0 & 1 & 1 & 0 \\
\hline
\end{tabular}


Case 3: Dichotomised advice network used for the clustering coefficient and density measure.

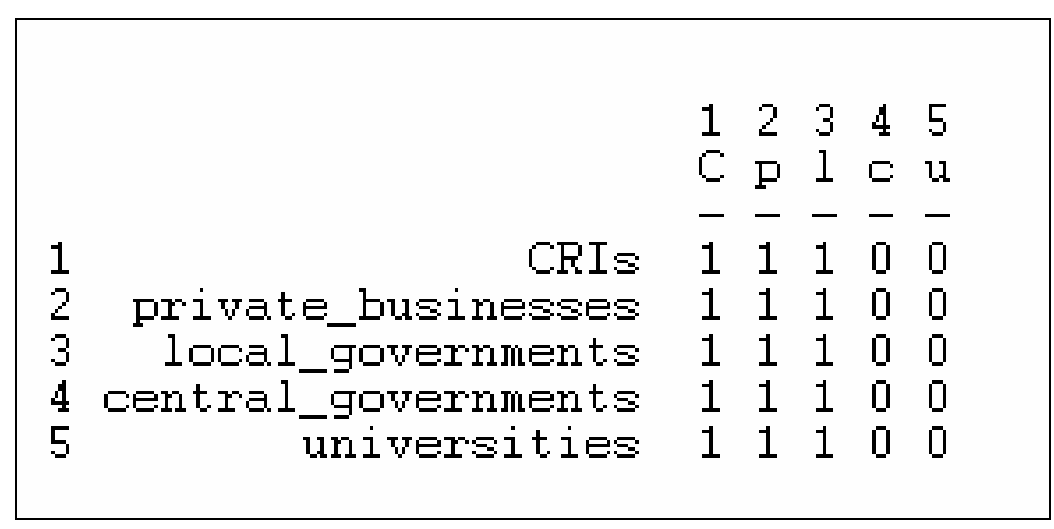

Case 3: comparable random network used for the clustering coefficient

\begin{tabular}{|lllllll|}
\hline & 1 & 2 & 3 & 4 & 5 & 6 \\
1 & $\frac{1}{1}$ & $\frac{1}{1}$ & $\overline{0}$ & $\frac{1}{1}$ & $\overline{0}$ & $\frac{1}{1}$ \\
2 & 1 & 1 & 0 & 1 & 1 & 1 \\
3 & 0 & 0 & 1 & 1 & 0 & 0 \\
4 & 0 & 0 & 0 & 1 & 0 & 0 \\
5 & 0 & 1 & 0 & 1 & 0 & 0 \\
6 & 1 & 1 & 1 & 1 & 1 & 1 \\
\hline
\end{tabular}


Appendix 20: cross-case analysis - intermediate ties data

\section{Intermediate ties in Case1}

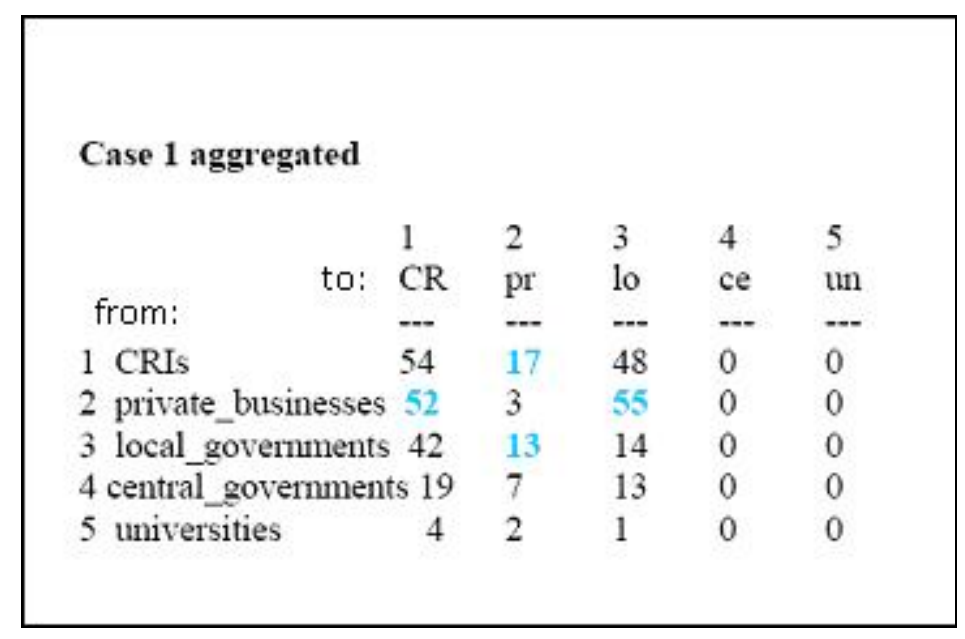

Case 1: uni-directional tie strength values

\section{$\underline{\text { K-means clustering results for Case 1: }}$}

QUICK CLUSTER VAR00001

/METHOD=KMEANS(NOUPDATE)

\begin{tabular}{|l|r|c|c|}
\hline \multicolumn{1}{|c|}{ Initial Cluster Centers } \\
\hline & \multicolumn{3}{|c|}{ Cluster } \\
\cline { 2 - 4 } & 1 & 2 & 3 \\
\hline VAR00001 & .00 & 90.00 & 69.00 \\
\hline
\end{tabular}




\begin{tabular}{|l|l|r|r|}
\hline Case & & & \\
Number & VAR00002 & Cluster & Distance \\
\hline 1 & weak & 1 & 7.500 \\
2 & inte & 1 & 5.500 \\
3 & stro & 1 & 3.500 \\
4 & & 1 & .500 \\
5 & & 1 & 5.500 \\
6 & & 1 & 11.500 \\
7 & & 3 & .500 \\
8 & & 3 & .500 \\
9 & & 2 & .000 \\
\hline
\end{tabular}

Final Cluster Centers

\begin{tabular}{|l|r|r|r|}
\hline \multirow{2}{*}{} & \multicolumn{3}{|c|}{ Cluster } \\
\cline { 2 - 4 } & \multicolumn{1}{|c|}{1} & \multicolumn{1}{|c|}{2} & 3 \\
\hline VAR00001 & 7.50 & 90.00 & 68.50 \\
\hline
\end{tabular}

Distances between Final Cluster Centers

\begin{tabular}{|l|l|l|l|}
\hline Cluster & 1 & 2 & 3 \\
\hline 1 & & 82.500 & 61.000 \\
2 & 82.500 & & 21.500 \\
3 & 61.000 & 21.500 & \\
\hline
\end{tabular}


Final classification of total tie strength values for Case 1

\begin{tabular}{|c|c|c|}
\hline $\begin{array}{c}\text { Total (reciprocal) } \\
\text { tie strength } \\
\text { values }\end{array}$ & Type of tie & Cluster \\
\hline $1(0+1)$ & Universities - local governments & weak \\
\hline $2(2+0)$ & Universities - private businesses & weak \\
\hline $4(0+4)$ & CRIs - universities & weak \\
\hline $7(7+0)$ & Private businesses - central governments & weak \\
\hline $13(13+0)$ & Local governments - central governments & weak \\
\hline $19(0+19)$ & CRls - central governments & weak \\
\hline $\mathbf{6 8}(\mathbf{5 5 + 1 3 )}$ & Private businesses - local governments & intermediate \\
\hline $\mathbf{6 9}(\mathbf{1 7 + 5 2})$ & CRIs - private businesses & intermediate \\
\hline $90(48+42)$ & CRIs - local governments & strong \\
\hline
\end{tabular}

\section{Intermediate ties in Case2}

Case 2 aggregated

\begin{tabular}{|c|c|c|c|c|c|c|c|}
\hline to: & $\stackrel{1}{\mathrm{CRI}}$ & $\begin{array}{l}2 \\
\text { cen }\end{array}$ & $\begin{array}{l}3 \\
\text { Uni }\end{array}$ & $\begin{array}{l}4 \\
\text { pri }\end{array}$ & $\begin{array}{l}5 \\
\text { loc }\end{array}$ & $\begin{array}{l}6 \\
\text { NPO }\end{array}$ & $\begin{array}{l}7 \\
\text { oth }\end{array}$ \\
\hline om: & - & -- & $\cdots$ & -- & $\cdots$ & -- & - \\
\hline $1 \mathrm{CRI}$ & 104 & 16 & 40 & 7 & 7 & 0 & 0 \\
\hline 2 central_government & 42 & 90 & 44 & 13 & 39 & 0 & 0 \\
\hline 3 Universities & 25 & 28 & 99 & 10 & 13 & 0 & 0 \\
\hline 4 private_businesses & 40 & 20 & 43 & 99 & 39 & 0 & 0 \\
\hline local_government & 1 & 5 & 1 & 2 & 14 & 0 & 0 \\
\hline $\mathrm{PO}$ & 0 & 0 & 8 & 0 & 0 & 0 & 0 \\
\hline other & 7 & 2 & 6 & 2 & 1 & 0 & 0 \\
\hline
\end{tabular}




\section{K-means clustering results for Case 2:}

QUICK CLUSTER VAR00001

/METHOD=KMEANS(NOUPDATE)

Initial Cluster Centers

\begin{tabular}{|l|r|r|r|}
\hline \multirow{2}{*}{} & \multicolumn{3}{|c|}{ Cluster } \\
\cline { 2 - 4 } & 1 & 2 & 3 \\
\hline VAR00001 & 1.00 & 33.00 & 72.00 \\
\hline
\end{tabular}

Cluster Membership

\begin{tabular}{|c|c|c|}
\hline $\begin{array}{l}\text { Case } \\
\text { Number }\end{array}$ & Cluster & Distance \\
\hline 1 & 1 & 5.000 \\
\hline 2 & 1 & 4.000 \\
\hline 3 & 1 & 4.000 \\
\hline 4 & 1 & .000 \\
\hline 5 & 1 & 1.000 \\
\hline 6 & 1 & 2.000 \\
\hline 7 & 1 & 2.000 \\
\hline 8 & 1 & 8.000 \\
\hline 9 & 2 & 8.250 \\
\hline 10 & 2 & .250 \\
\hline 11 & 2 & 2.750 \\
\hline 12 & 2 & 5.750 \\
\hline 13 & 3 & 9.000 \\
\hline 14 & 3 & 4.000 \\
\hline 15 & 3 & 3.000 \\
\hline 16 & 3 & 10.000 \\
\hline
\end{tabular}


Final Cluster Centers

\begin{tabular}{|l|r|r|r|}
\hline \multirow{2}{*}{} & \multicolumn{3}{|c|}{ Cluster } \\
\cline { 2 - 4 } & 1 & \multicolumn{1}{|c|}{2} & \multicolumn{1}{c|}{3} \\
\hline VAR00001 & 6.00 & 41.25 & 62.00 \\
\hline
\end{tabular}

Distances between Final Cluster Centers

\begin{tabular}{|l|l|l|l|}
\hline Cluster & 1 & 2 & 3 \\
\hline 1 & & 35.250 & 56.000 \\
2 & 35.250 & & 20.750 \\
3 & 56.000 & 20.750 & \\
\hline
\end{tabular}

Intermediate ties in Case 3

\begin{tabular}{|c|c|c|c|c|c|c|}
\hline \multicolumn{7}{|l|}{ Case 3 aggregated } \\
\hline & 1 & 2 & 3 & 4 & 5 & 6 \\
\hline to: & CR & un & $\mathrm{pr}$ & $\mathrm{ce}$ & lo & ot \\
\hline trom: & -- & -- & $\ldots$ & -- & $\cdots$ & - \\
\hline 1 CRIs & 49 & 22 & 19 & 23 & 30 & 0 \\
\hline 2 universities & 6 & 26 & 8 & 13 & 14 & 0 \\
\hline 3 private_businesses & 17 & 16 & 31 & 29 & 25 & 0 \\
\hline 4 central government & 9 & 6 & 14 & 48 & 25 & 0 \\
\hline 5 local_government & 26 & 20 & 35 & 36 & 42 & 0 \\
\hline 6 others & 1 & 1 & 0 & 0 & 2 & 0 \\
\hline
\end{tabular}

Case 3: uni-directional tie strength values 


\section{$\underline{\text { K-means clustering results for Case 3: }}$}

QUICK CLUSTER VAR00001

/METHOD=KMEANS(NOUPDATE)

\begin{tabular}{|l|r|r|r|}
\multicolumn{4}{|c|}{ Initial Cluster Centers } \\
\hline & \multicolumn{3}{|c|}{ Cluster } \\
\cline { 2 - 4 } & 1 & 2 & 3 \\
\hline VAR00001 & 1.00 & 19.00 & 61.00 \\
\hline
\end{tabular}

\begin{tabular}{|l|r|r|}
\hline \multicolumn{2}{|c|}{ Cluster Membership } \\
\hline Case & \multicolumn{1}{c|}{} \\
Number & Cluster & Distance \\
\hline 1 & 1 & .333 \\
2 & 1 & .333 \\
3 & 1 & .667 \\
4 & 2 & 9.833 \\
5 & 2 & 4.833 \\
6 & 2 & .833 \\
7 & 2 & 3.167 \\
8 & 2 & 5.167 \\
9 & 2 & 7.167 \\
10 & 3 & 12.000 \\
11 & 3 & 1.000 \\
12 & 3 & 5.000 \\
13 & 3 & 6.000 \\
\hline
\end{tabular}

Final Cluster Centers

\begin{tabular}{|l|r|r|r|}
\hline \multirow{2}{*}{} & \multicolumn{3}{|c|}{ Cluster } \\
\cline { 2 - 4 } & 1 & 2 & \multicolumn{1}{c|}{3} \\
\hline VAR00001 & 1.33 & 28.83 & 55.00 \\
\hline
\end{tabular}


Distances between Final Cluster Centers

\begin{tabular}{|l|l|l|l|}
\hline Cluster & 1 & 2 & 3 \\
\hline 1 & & 27.500 & 53.667 \\
2 & 27.500 & & 26.167 \\
3 & 53.667 & 26.167 & \\
\hline
\end{tabular}

Final classification of total tie strength values for Case 3

\begin{tabular}{|c|c|c|}
\hline $\begin{array}{c}\text { Total } \\
\text { (reciprocal) tie } \\
\text { strength values }\end{array}$ & Type of tie & Cluster \\
\hline $1(0+1)$ & CRIs - others & weak \\
\hline $1(0+1)$ & Universities - others & weak \\
\hline $2(0+2)$ & Local governments - others & weak \\
\hline $19(13+6)$ & Universities - central governments & intermediate \\
\hline $24(8+16)$ & Universities - private businesses & intermediate \\
\hline $28(22+6)$ & CRIs - universities & intermediate \\
\hline $32(23+9)$ & CRIs - central governments & intermediate \\
\hline $34(14+20)$ & Universities - local governments & intermediate \\
\hline $36(19+17)$ & CRIs - private businesses & intermediate \\
\hline $43(29+14)$ & Private businesses - central governments & intermediate \\
\hline $56(30+26)$ & CRIs - local governments & strong \\
\hline $60(25+35)$ & Private businesses - local governments & strong \\
\hline $61(36+25)$ & Local governments - central governments & strong \\
\hline
\end{tabular}


Appendix 21: cross-case analysis - non-aggregated intermediate ties data per case

Social network data (at the organisational level) supporting intermediate ties among groups of organisation in the small-world networks

Coding for levels of contract frequency:

\begin{tabular}{|l|l|}
\hline frequency & coding \\
\hline & \\
\hline at least once every week & 4 \\
\hline at least once every month & 3 \\
\hline at least once every 3 months & 2 \\
\hline at least once every 6 months & 1 \\
\hline never & 0 \\
\hline
\end{tabular}

In regard to the contact frequencies and the number of study participants, the following file extract shows the non-aggregated survey data for the advice network. The 'labels' indicate all possible knowledge givers and receivers in this case. These are five in this case $(\mathrm{n}=5)$. In an ascending order, they can be numbered (coded) 1 to 5. The respective code appears in brackets after each group of organisation, e.g. CRIs (1). This means CRIs are coded with a 1 in the following table.

The following numbers represent the coded knowledge givers, the coded knowledge seekers and the coded contact frequencies. The first number in each set of three numbers in the table below indicates the coded knowledge giver whereas the second number indicates the coded knowledge receiver. The third number shows the coded contact frequency. For example, the first set of numbers $\left(\begin{array}{lll}4 & 2 & 1\end{array}\right)$ indicates central government (4) as the knowledge giver, private businesses (3) as the knowledge receivers and a relatively low contact frequency of at least once every 6 months (1).

\section{Intermediate ties - Case 1: five groups of organisation}

$\mathrm{dl} n=5$ format $=$ edgelist 1

labels:

CRIs (1) ,private_businesses (2), local_governments (3), central_governments (4) ,universities (5)

data:

421

432

411 
422

433

411

413

432

411

411

421

411

421

433

412

412

422

433

411

413

Intermediate ties - Case 2: seven groups of organisation $\mathrm{dl} n=7$ format $=$ edgelist 1

labels: CRIs (1) ,central_government (2),Universities (3),private_businesses (4) ,local_government (5),NPO (6), other (7)

data:

$\begin{array}{lll}240 & 421 & 351 \\ 240 & 150 & 350 \\ 420 & 150 & 350 \\ 240 & 150 & 353 \\ 240 & 240 & 511 \\ 421 & 240 & 530 \\ 421 & 241 & 634 \\ 240 & 240 & 150\end{array}$




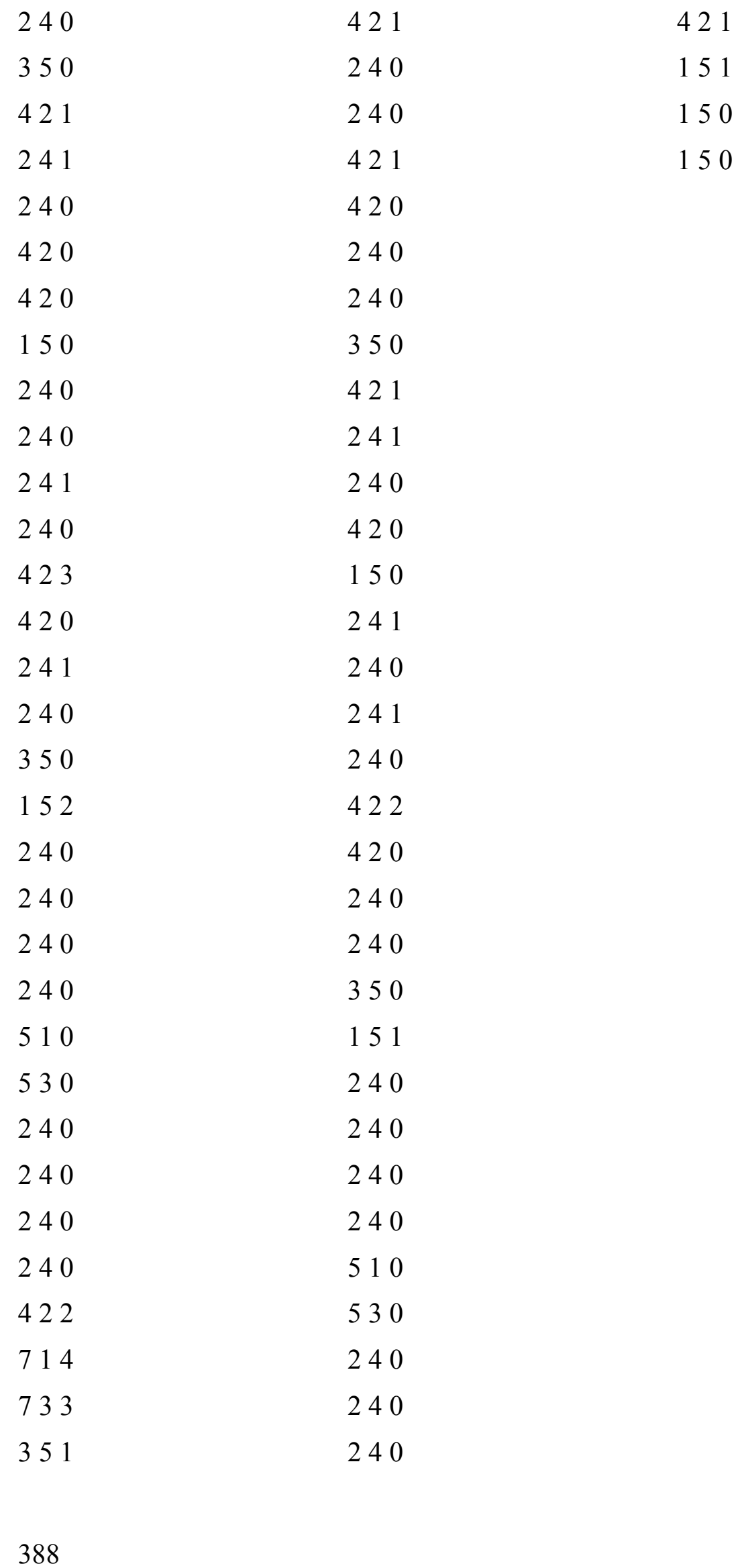




$\begin{array}{ll}152 & 240 \\ 350 & 421 \\ 150 & 713 \\ 150 & 733 \\ 350 & 351 \\ 242 & 350 \\ 420 & 150 \\ 350 & 151 \\ 351 & 350 \\ 350 & 243 \\ 351 & 422 \\ 350 & 351 \\ 240 & 350 \\ 240 & 352 \\ 352 & \\ 510 & \\ 531 & \\ 634 & \\ 150 & \\ 422 & \end{array}$

Intermediate ties - Case 3: six groups of organisation

$\mathrm{dl} n=6$ format $=$ edgelist 1

labels: CRIs (1), universities (2) ,private_businesses (3) ,central_government (4) ,local_government (5), others (6)

data:

521

310

321

523

242

421 


\begin{tabular}{|c|c|c|}
\hline 520 & 251 & 311 \\
\hline 421 & 520 & 321 \\
\hline 233 & 131 & 322 \\
\hline 241 & 420 & 240 \\
\hline 241 & 522 & 241 \\
\hline 253 & 421 & 520 \\
\hline 420 & 520 & 311 \\
\hline 231 & 523 & 320 \\
\hline 240 & 520 & 230 \\
\hline 240 & 421 & 242 \\
\hline 251 & 231 & 241 \\
\hline 522 & 240 & 252 \\
\hline 134 & 242 & 520 \\
\hline 130 & 252 & 130 \\
\hline 520 & 421 & 420 \\
\hline 520 & 420 & 522 \\
\hline 132 & 232 & \\
\hline 131 & 241 & \\
\hline 310 & 241 & \\
\hline 321 & 252 & \\
\hline 421 & 521 & \\
\hline 420 & 131 & \\
\hline 520 & 130 & \\
\hline 132 & 521 & \\
\hline 133 & 520 & \\
\hline 522 & 130 & \\
\hline 521 & 130 & \\
\hline 312 & 310 & \\
\hline 320 & 320 & \\
\hline 311 & 420 & \\
\hline 324 & 420 & \\
\hline 313 & 520 & \\
\hline
\end{tabular}




$\begin{array}{ll}321 & 130 \\ 420 & 133 \\ 311 & 132 \\ 321 & 521 \\ 231 & 322 \\ 240 & 521 \\ 241 & 312 \\ 252 & 320 \\ 520 & 314 \\ 311 & 323 \\ & 311\end{array}$


Appendix 22: cross case analysis - Case 2 tie strength distribution

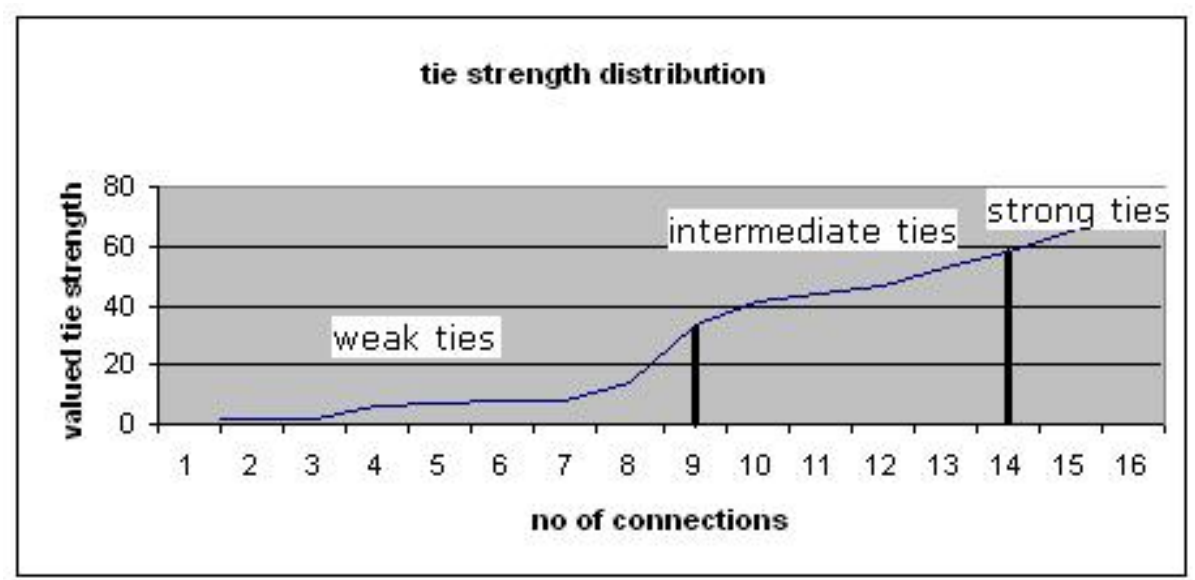


Appendix 23: cross-case analysis: univariate statistics results per case at the group of organisation level

Case 1: Summary of univariate statistics for key groups of organisation by column (received relationships)

\begin{tabular}{|l|l|l|l|l|l|}
\hline & CRIs & $\begin{array}{l}\text { Private } \\
\text { businesses }\end{array}$ & $\begin{array}{l}\text { Local } \\
\text { governments }\end{array}$ & $\begin{array}{l}\text { Central } \\
\text { governments }\end{array}$ & Universities \\
\hline Mean & 29.25 & 9.75 & 29.25 & 0.00 & 0.00 \\
\hline Std Dev & 18.86 & 5.72 & 22.79 & 0.00 & 0.00 \\
\hline Sum & 117.00 & 39.00 & 117.00 & 0.00 & 0.00 \\
\hline Variance & 355.69 & 32.69 & 519.19 & 0.00 & 0.00 \\
\hline Minimum & 4.00 & 2.00 & 1.00 & 0.00 & 0.00 \\
\hline Maximum & 52.00 & 17.00 & 55.00 & 0.00 & 0.00 \\
\hline No of Obs & 4.00 & 4.00 & 4.00 & 4.00 & 4.00 \\
\hline
\end{tabular}

Case 1: Summary of univariate statistics for key groups of organisation by row (initiated relationships)

\begin{tabular}{|l|l|l|l|l|l|}
\hline & CRIs & $\begin{array}{l}\text { Private } \\
\text { businesses }\end{array}$ & $\begin{array}{l}\text { Local } \\
\text { governments }\end{array}$ & $\begin{array}{l}\text { Central } \\
\text { governments }\end{array}$ & Universities \\
\hline Mean & 16.25 & 26.75 & 13.75 & 9.75 & 1.75 \\
\hline Std Dev & 19.60 & 26.77 & 17.15 & 7.05 & 1.48 \\
\hline Sum & 65.00 & 107.00 & 55.00 & 39.00 & 7.00 \\
\hline Variance & 384.19 & 716.69 & 294.19 & 49.69 & 2.19 \\
\hline Minimum & 0.00 & 0.00 & 0.00 & 0.00 & 0.00 \\
\hline Maximum & 48.00 & 55.00 & 42.00 & 19.00 & 4.00 \\
\hline No of Obs & 4.00 & 4.00 & 4.00 & 4.00 & 4.00 \\
\hline
\end{tabular}


Case 2: Table: Summary of univariate statistics for groups of organisations by column (received relationships)

\begin{tabular}{|l|l|l|l|l|l|l|l|}
\hline & CRIs & $\begin{array}{l}\text { Central } \\
\text { governments }\end{array}$ & Universities & $\begin{array}{l}\text { Private } \\
\text { businesses }\end{array}$ & $\begin{array}{l}\text { Local } \\
\text { governments }\end{array}$ & NPOs & others \\
\hline Mean & 19.17 & 11.83 & 23.67 & 5.67 & 16.50 & 0.00 & 0.00 \\
\hline Std Dev & 17.49 & 10.24 & 18.82 & 4.71 & 16.47 & 0.00 & 0.00 \\
\hline Sum & 115.00 & 71.00 & 142.000 & 34.00 & 99.00 & 0.00 & 0.00 \\
\hline Variance & 305.81 & 104.81 & 354.22 & 22.22 & 271.25 & 0.00 & 0.00 \\
\hline Minimum & 0.00 & 0.00 & 1.00 & 0.00 & 0.00 & 0.00 & 0.00 \\
\hline Maximum & 42.00 & 28.00 & 44.00 & 13.00 & 39.00 & 0.00 & 0.00 \\
\hline $\begin{array}{l}\text { No of } \\
\text { Obs }\end{array}$ & 6.00 & 6.00 & 6.00 & 6.00 & 6.00 & 6.00 & 6.00 \\
\hline
\end{tabular}

Case 2: Table: Summary of univariate statistics for groups of organisations by row (initiated relationships)

\begin{tabular}{|l|l|l|l|l|l|l|l|}
\hline & CRIs & $\begin{array}{l}\text { Central } \\
\text { governments }\end{array}$ & Universities & $\begin{array}{l}\text { Private } \\
\text { businesses }\end{array}$ & $\begin{array}{l}\text { Local } \\
\text { governments }\end{array}$ & NPOs & others \\
\hline Mean & 11.67 & 23.00 & 12.67 & 23.67 & 1.50 & 1.33 & 3.00 \\
\hline Std Dev & 13.77 & 19.22 & 10.92 & 18.30 & 1.71 & 2.98 & 2.58 \\
\hline Sum & 70.00 & 138.00 & 76.00 & 142.00 & 9.00 & 8.00 & 18.00 \\
\hline Variance & 189.56 & 369.33 & 119.22 & 334.89 & 2.92 & 8.89 & 6.67 \\
\hline Minimum & 0.00 & 0.00 & 0.00 & 0.00 & 0.00 & 0.00 & 0.00 \\
\hline Maximum & 40.00 & 44.00 & 28.00 & 43.00 & 5.00 & 8.00 & 7.00 \\
\hline $\begin{array}{l}\text { No of } \\
\text { Obs }\end{array}$ & 6.00 & 6.00 & 6.00 & 6.00 & 6.00 & 6.00 & 6.00 \\
\hline
\end{tabular}


Case 3: Table: Summary of univariate statistics for groups of organisations by column (received relationships)

\begin{tabular}{|l|l|l|l|l|l|l|}
\hline & CRIs & Universities & $\begin{array}{l}\text { Private } \\
\text { businesses }\end{array}$ & $\begin{array}{l}\text { Central } \\
\text { governments }\end{array}$ & $\begin{array}{l}\text { Local } \\
\text { governments }\end{array}$ & others \\
\hline Mean & 11.80 & 13.00 & 15.20 & 20.20 & 19.20 & 0.00 \\
\hline Std Dev & 8.795 & 8.149 & 11.754 & 12.608 & 10.068 & 0.00 \\
\hline Sum & 59.00 & 65.00 & 76.00 & 101.00 & 96.00 & 0.00 \\
\hline Variance & 77.36 & 66.40 & 138.16 & 158.96 & 101.36 & 0.00 \\
\hline Minimum & 1.00 & 1.00 & 0.00 & 0.00 & 2.00 & 0.00 \\
\hline Maximum & 26.00 & 22.00 & 35.00 & 36.0 & 30.00 & 0.00 \\
\hline $\begin{array}{l}\text { No of } \\
\text { Obs }\end{array}$ & 5.00 & 5.00 & 5.00 & 5.00 & 5.00 & 5.00 \\
\hline
\end{tabular}

Case 3: Table: Summary of univariate statistics for groups of organisations by row (initiated relationships)

\begin{tabular}{|l|l|l|l|l|l|l|}
\hline & CRIs & Universities & $\begin{array}{l}\text { Private } \\
\text { businesses }\end{array}$ & $\begin{array}{l}\text { Central } \\
\text { governments }\end{array}$ & $\begin{array}{l}\text { Local } \\
\text { governments }\end{array}$ & others \\
\hline Mean & 18.80 & 8.20 & 17.40 & 10.80 & 23.40 & 0.80 \\
\hline Std Dev & 10.07 & 5.07 & 9.97 & 8.42 & 13.11 & 0.75 \\
\hline Sum & 94.00 & 41.00 & 87.00 & 54.00 & 117.00 & 4.00 \\
\hline Variance & 101.36 & 25.76 & 99.44 & 70.96 & 171.84 & 0.56 \\
\hline Minimum & 0.00 & 0.00 & 0.00 & 0.00 & 0.00 & 0.00 \\
\hline Maximum & 30.00 & 14.00 & 29.00 & 25.00 & 36.00 & 2.00 \\
\hline $\begin{array}{l}\text { No of } \\
\text { Obs }\end{array}$ & 5.00 & 5.00 & 5.00 & 5.00 & 5.00 & 5.00 \\
\hline
\end{tabular}


Appendix 24: cross-case analysis - information centrality scores per case

This table shows the Information Centrality score for each group of organisation per case.

\begin{tabular}{|l|l|l|}
\hline Case 1 & Case 2 & Case 3 \\
\hline CRIs $=25.45$ & Universities $=30.13$ & Local government $=19.12$ \\
\hline $\begin{array}{l}\text { Private businesses }= \\
24.62\end{array}$ & Central governments $=28.61$ & CRIs $=18.46$ \\
\hline $\begin{array}{l}\text { Local governments }= \\
24.60\end{array}$ & CRIs $=28.54$ & Central governments $=18.31$ \\
\hline $\begin{array}{l}\text { Central governments }= \\
19.35\end{array}$ & Private businesses $=28.53$ & Private businesses $=18.28$ \\
\hline Universities $=8.18$ & Local governments $=26.32$ & Universities $=17.76$ \\
\hline & Others $=14.27$ & Others $=4.65$ \\
\hline & NPO $=8.16$ & \\
\hline
\end{tabular}




\section{Appendix 25: cross-case analysis - preferred types of knowledge transferred by tie strength in Case 2 and Case 3}

Case 2: types of knowledge transferred by weak and strong ties for giving and seeking advice activities.

\section{Weak ties:}

\begin{tabular}{|c|c|c|c|c|}
\hline & $\begin{array}{l}\text { electronically } \\
\text { accessible }\end{array}$ & $\begin{array}{c}\text { electronically } \\
\text { inaccessible (but } \\
\text { codified) }\end{array}$ & non-codified (tacit) ${ }^{*}$ & $\begin{array}{l}\text { Response } \\
\text { Count }\end{array}$ \\
\hline an expert's contact details & $80.0 \%(4)$ & $20.0 \%(1)$ & $20.0 \%(1)$ & 5 \\
\hline $\begin{array}{r}\text { a reference to another source of } \\
\text { information }\end{array}$ & $66.7 \%(4)$ & $33.3 \%(2)$ & $16.7 \%(1)$ & 6 \\
\hline formal information(how-to expertise) & $100.0 \%(3)$ & $0.0 \%(0)$ & $66.7 \%(2)$ & 3 \\
\hline formal information(facts) & $100.0 \%(4)$ & $25.0 \%(1)$ & $50.0 \%(2)$ & 4 \\
\hline $\begin{array}{r}\text { informal information (how-to } \\
\text { expertise) }\end{array}$ & $50.0 \%(2)$ & $25.0 \%(1)$ & $75.0 \%(3)$ & 4 \\
\hline informal information (facts) & $50.0 \%(2)$ & $25.0 \%(1)$ & $75.0 \%(3)$ & 4 \\
\hline \multicolumn{4}{|c|}{$\begin{array}{r}{ }^{*} \text { non-codified (tacit) content refers to the knowledge that people carry in their minds, but which is not written down (as } \\
\text { codified knowledge is). Other (please specify below) }\end{array}$} & 0 \\
\hline & & & answered question & 7 \\
\hline
\end{tabular}

14. Please indicate a maximum of three most important types of content that are communicated when you seek advice from colleagues from other organisations.

\begin{tabular}{|c|c|c|c|c|}
\hline & $\begin{array}{l}\text { electronically } \\
\text { accessible }\end{array}$ & $\begin{array}{c}\text { electronically } \\
\text { inaccessible (but } \\
\text { codified) }\end{array}$ & non-codified (tacit) ${ }^{\star}$ & $\begin{array}{l}\text { Response } \\
\text { Count }\end{array}$ \\
\hline an expert's contact details & $100.0 \%(4)$ & $0.0 \%(0)$ & $0.0 \%(0)$ & 4 \\
\hline $\begin{array}{r}\text { a reference to another source of } \\
\text { information }\end{array}$ & $100.0 \%(3)$ & $0.0 \%(0)$ & $0.0 \%(0)$ & 3 \\
\hline $\begin{array}{r}\text { formal information (how-to } \\
\text { expertise) }\end{array}$ & $75.0 \%(3)$ & $0.0 \%(0)$ & $50.0 \%(2)$ & 4 \\
\hline formal information (facts) & $80.0 \%(4)$ & $0.0 \%(0)$ & $40.0 \%(2)$ & 5 \\
\hline $\begin{array}{r}\text { informal information (how-to } \\
\text { expertise) }\end{array}$ & $100.0 \%(3)$ & $0.0 \%(0)$ & $33.3 \%(1)$ & 3 \\
\hline informal information (facts) & $100.0 \%(2)$ & $0.0 \%(0)$ & $50.0 \%(1)$ & 2 \\
\hline ntent refe & edge that & $\begin{array}{l}\text { in their minds, } b \\
\text { fied knowledge is }\end{array}$ & $\begin{array}{l}\text { S not written down (as } \\
\text { please specify below) }\end{array}$ & 0 \\
\hline
\end{tabular}




\section{Strong ties:}

\begin{tabular}{|c|c|c|c|c|}
\hline \multicolumn{5}{|c|}{$\begin{array}{l}\text { 11. Please indicate a maximum of three most important types of content that are communicated when you give advice to } \\
\text { colleagues from other organisations. }\end{array}$} \\
\hline & $\begin{array}{l}\text { electronically } \\
\text { accessible }\end{array}$ & $\begin{array}{l}\text { electronically } \\
\text { inaccessible (but } \\
\text { codified) }\end{array}$ & non-codified (tacit) ${ }^{*}$ & $\begin{array}{l}\text { Response } \\
\text { Count }\end{array}$ \\
\hline an expert's contact details & $84.6 \%(22)$ & $11.5 \%(3)$ & $11.5 \%(3)$ & 26 \\
\hline $\begin{array}{r}\text { a reference to another source of } \\
\text { information }\end{array}$ & $77.8 \%(28)$ & $27.8 \%(10)$ & $19.4 \%(7)$ & 36 \\
\hline formal information(how-to expertise) & $78.6 \%(22)$ & $14.3 \%(4)$ & $25.0 \%(7)$ & 28 \\
\hline formal information(facts) & $76.5 \%(26)$ & $26.5 \%(9)$ & $20.6 \%(7)$ & 34 \\
\hline $\begin{array}{r}\text { informal information (how-to } \\
\text { expertise) }\end{array}$ & $43.3 \%(13)$ & $10.0 \%(3)$ & $76.7 \%(23)$ & 30 \\
\hline informal information (facts) & $52.0 \%(13)$ & $24.0 \%(6)$ & $60.0 \%(15)$ & 25 \\
\hline \multicolumn{4}{|c|}{$\begin{array}{r}\text { * non-codified (tacit) content refers to the knowledge that people carry in their minds, but which is not written down (as } \\
\text { codified knowledge is). Other (please specify below) }\end{array}$} & 1 \\
\hline & & & on & 55 \\
\hline
\end{tabular}

14. Please indicate a maximum of three most important types of content that are communicated when you seek advice from colleagues from other organisations.

\begin{tabular}{|c|c|c|c|}
\hline $\begin{array}{l}\text { a reference to another source of } \\
\text { information }\end{array}$ & $84.4 \%(27)$ & $21.9 \%(7)$ & $15.6 \%(5)$ \\
\hline $\begin{array}{r}\text { formal information (how-to } \\
\text { expertise) }\end{array}$ & $60.0 \%(15)$ & $24.0 \%(6)$ & $52.0 \%(13)$ \\
\hline formal information (facts) & $76.5 \%(26)$ & $29.4 \%(10)$ & $14.7 \%(5)$ \\
\hline $\begin{array}{r}\text { informal information (how-to } \\
\text { expertise) }\end{array}$ & $48.7 \%(19)$ & $12.8 \%(5)$ & $66.7 \%(26)$ \\
\hline informal information (facts) & $63.6 \%(14)$ & $18.2 \%(4)$ & $45.5 \%(10)$ \\
\hline & & knowledge & ase specify below) \\
\hline & & & nswered question \\
\hline
\end{tabular}


Case 3: types of knowledge transferred by weak and strong ties for giving and seeking advice activities.

\section{Weak ties:}

\begin{tabular}{|c|c|c|c|c|}
\hline & $\begin{array}{l}\text { electronically } \\
\text { accessible }\end{array}$ & $\begin{array}{c}\text { electronically } \\
\text { inaccessible (but } \\
\text { codified) }\end{array}$ & non-codified (tacit) ${ }^{*}$ & $\begin{array}{l}\text { Response } \\
\text { Count }\end{array}$ \\
\hline an expert's contact details & $60.0 \%(3)$ & $60.0 \%(3)$ & $80.0 \%(4)$ & 5 \\
\hline $\begin{array}{r}\text { a reference to another source of } \\
\text { information }\end{array}$ & $66.7 \%(4)$ & $66.7 \%(4)$ & $66.7 \%(4)$ & 6 \\
\hline formal information(how-to expertise) & $75.0 \%(3)$ & $50.0 \%(2)$ & $50.0 \%(2)$ & 4 \\
\hline formal information(facts) & $80.0 \%(4)$ & $60.0 \%(3)$ & $40.0 \%(2)$ & 5 \\
\hline $\begin{array}{r}\text { informal information (how-to } \\
\text { expertise) }\end{array}$ & $80.0 \%(4)$ & $20.0 \%(1)$ & $60.0 \%(3)$ & 5 \\
\hline informal information (facts) & $50.0 \%(3)$ & $50.0 \%(3)$ & $66.7 \%(4)$ & 6 \\
\hline \multicolumn{4}{|c|}{$\begin{array}{r}\text { * non-codified (tacit) content refers to the knowledge that people carry in their minds, but which is not written down (as } \\
\text { codified knowledge is). Other (please specify below) }\end{array}$} & 1 \\
\hline & & & answered question & 6 \\
\hline
\end{tabular}

\begin{tabular}{|c|c|c|c|c|}
\hline & $\begin{array}{l}\text { electronically } \\
\text { accessible }\end{array}$ & $\begin{array}{c}\text { electronically } \\
\text { inaccessible (but } \\
\text { codified) }\end{array}$ & non-codified (tacit) ${ }^{\star}$ & $\begin{array}{l}\text { Response } \\
\text { Count }\end{array}$ \\
\hline an expert's contact details & $80.0 \%(4)$ & $40.0 \%(2)$ & $20.0 \%(1)$ & 5 \\
\hline $\begin{array}{r}\text { a reference to another source of } \\
\text { information }\end{array}$ & $85.7 \%(6)$ & $28.6 \%(2)$ & $14.3 \%(1)$ & 7 \\
\hline $\begin{array}{r}\text { formal information (how-to } \\
\text { expertise) }\end{array}$ & $100.0 \%(3)$ & $33.3 \%(1)$ & $33.3 \%(1)$ & 3 \\
\hline formal information (facts) & $100.0 \%(6)$ & $33.3 \%(2)$ & $16.7 \%(1)$ & 6 \\
\hline $\begin{array}{r}\text { informal information (how-to } \\
\text { expertise) }\end{array}$ & $60.0 \%(3)$ & $40.0 \%(2)$ & $40.0 \%(2)$ & 5 \\
\hline informal information (facts) & $60.0 \%(3)$ & $80.0 \%(4)$ & $20.0 \%(1)$ & 5 \\
\hline \multicolumn{4}{|c|}{$\begin{array}{r}{ }^{*} \text { non-codified (tacit) content refers to the knowledge that people carry in their minds, but which is not written down (as } \\
\text { codified knowledge is). Other (please specify below) }\end{array}$} & 0 \\
\hline
\end{tabular}




\section{Strong ties:}

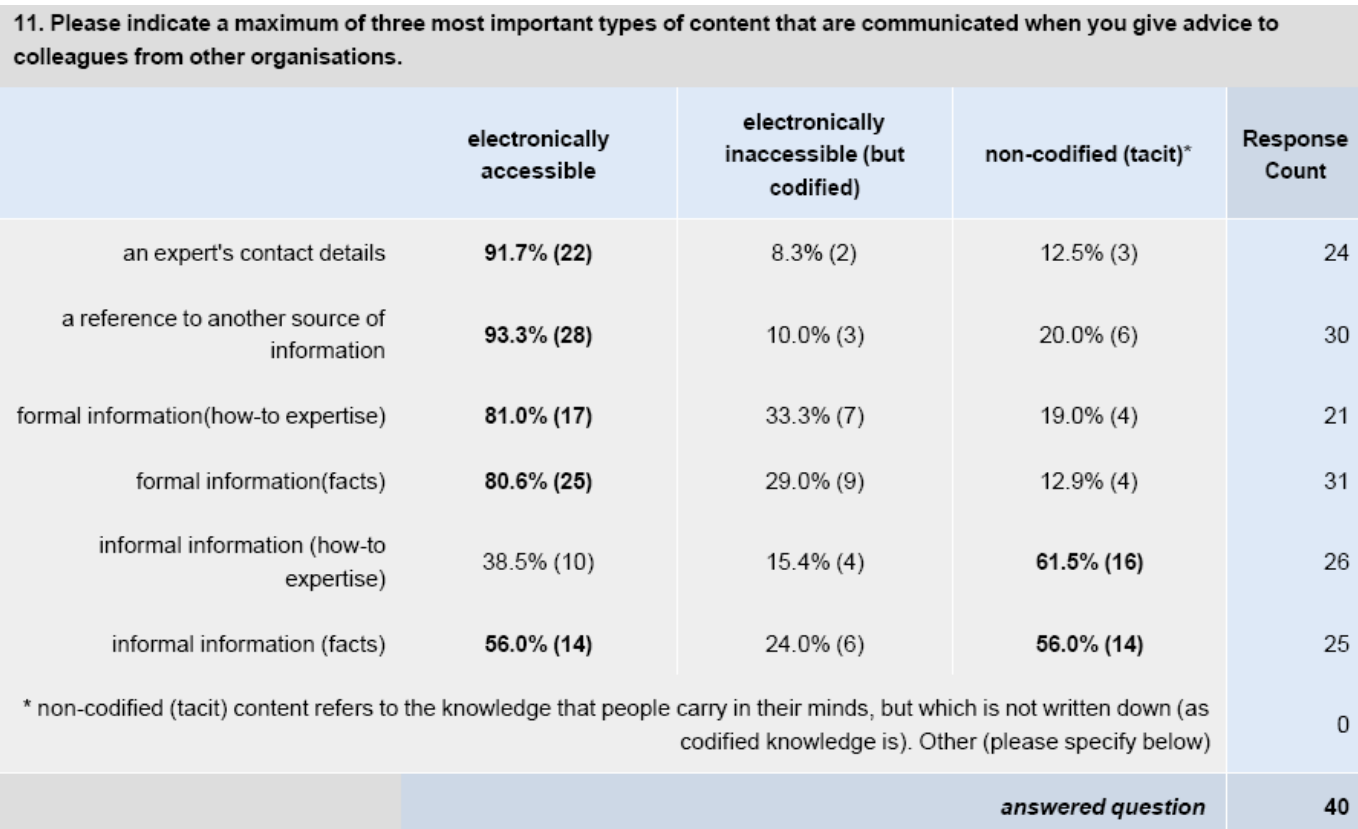

14. Please indicate a maximum of three most important types of content that are communicated when you seek advice from colleagues from other organisations.

\begin{tabular}{|c|c|c|c|c|}
\hline & $\begin{array}{l}\text { electronically } \\
\text { accessible }\end{array}$ & $\begin{array}{c}\text { electronically } \\
\text { inaccessible (but } \\
\text { codified) }\end{array}$ & non-codified (tacit) ${ }^{\star}$ & $\begin{array}{l}\text { Response } \\
\text { Count }\end{array}$ \\
\hline an expert's contact details & $95.2 \%(20)$ & $4.8 \%(1)$ & $14.3 \%(3)$ & 21 \\
\hline $\begin{array}{r}\text { a reference to another source of } \\
\text { information }\end{array}$ & $90.3 \%(28)$ & $9.7 \%(3)$ & $16.1 \%(5)$ & 31 \\
\hline $\begin{array}{r}\text { formal information (how-to } \\
\text { expertise) }\end{array}$ & $84.2 \%(16)$ & $10.5 \%(2)$ & $15.8 \%(3)$ & 19 \\
\hline formal information (facts) & $61.3 \%(19)$ & $25.8 \%(8)$ & $38.7 \%(12)$ & 31 \\
\hline $\begin{array}{r}\text { informal information (how-to } \\
\text { expertise) }\end{array}$ & $52.9 \%(9)$ & $17.6 \%(3)$ & $58.8 \%(10)$ & 17 \\
\hline informal information (facts) & $38.1 \%(8)$ & $23.8 \%(5)$ & $66.7 \%(14)$ & 21 \\
\hline \multicolumn{4}{|c|}{$\begin{array}{r}\text { * non-codified (tacit) content refers to the knowledge that people carry in their minds, but which is not written down (as } \\
\text { codified knowledge is). Other (please specify below) }\end{array}$} & 1 \\
\hline & & & answ & 38 \\
\hline
\end{tabular}




\section{Appendix 26: Case 1 survey results - media/ICT use}

Media/ICT use differentiated by two phases: initial contact and follow-up contact

$\begin{array}{ll} & \text { no of hits by survey } \\ \text { initial contact } & \text { participants } \\ \text { email } & 81 \\ \text { phone } & 42 \\ \text { meeting } & 28 \\ \text { workshop/seminar/conference } & 11 \\ \text { video-conferencing } & 0 \\ \text { online discussion forum } & 0 \\ \text { blog } & 0 \\ \text { wiki } & 0 \\ & \\ \text { follow-up contact } & \\ \text { email } & 129 \\ \text { phone } & 94 \\ \text { meeting } & 61 \\ \text { workshop/seminar/conf } & 12 \\ \text { video-conferencing } & 2 \\ \text { blog } & 0 \\ \text { online discussion forum } & 0 \\ \text { wiki } & 0\end{array}$




\section{Appendix 27: Case 2 survey results - media/ICT use}

Media/ICT use differentiated by three phases: initial contact, follow-up contact, maintenance

$\begin{array}{ll} & \text { no of hits by survey } \\ \text { initial contact } & \text { participants } \\ \text { email } & 230 \\ \text { phone } & 105 \\ \text { meeting } & 58 \\ \text { workshop/seminar } & 55 \\ \text { other: conference, lunch, casual meeting, } & 6 \\ \text { video-conferencing } & 3 \\ \text { online discussion forum } & 2 \\ \text { blog } & 1 \\ \text { wiki } & 0 \\ & \\ \text { follow-up contact } & \\ \text { email } & 208 \\ \text { phone } & 130 \\ \text { meeting } & 114 \\ \text { workshop/seminar } & 19 \\ \text { video-conferencing } & 2 \\ \text { other:face-to-face } & 1 \\ \text { blog } & 0 \\ \text { online discussion forum } & 0 \\ \text { wiki } & 0 \\ & \end{array}$




\section{Appendix 28: Case 3 survey results - media/ICT use}

Media/ICT use differentiated by three phases: initial contact, follow-up contact, maintenance

\begin{tabular}{|l|l|l|l|}
\hline Media & Initial (hits) & follow-up (hits) & maintain (hits) \\
\hline email & 131 & 171 & 181 \\
\hline phone & 94 & 104 & 98 \\
\hline meeting & 59 & 63 & 33 \\
\hline workshop/seminar & 53 & 8 & 9 \\
\hline online discussion forum & 3 & 2 & 1 \\
\hline video-conferencing & 1 & 1 & 1 \\
\hline wiki & 0 & 0 & 0 \\
\hline blog & 0 & 6 & 2 \\
\hline
\end{tabular}




\section{$\underline{\text { Appendix 29: Case } 1 \text { survey results - media/ICT use by tie strength }}$}

Media/ICT use differentiated by strong ties and weak ties and the three phases of initial contact and follow-up contact

\begin{tabular}{llll} 
Strong ties & \multicolumn{3}{l}{ Weak ties } \\
initial contact & no of hits & initial contact & no of hits \\
email & 45 & email & 37 \\
phone & 18 & phone & 32 \\
meeting & 18 & meeting & 11 \\
workshop/seminar/conf & 5 & workshop/seminar/conf & 7 \\
video-conferencing & 0 & video-conferencing & 0 \\
online discussion forum & 0 & online discussion forum & 0 \\
blog & 0 & blog & 0 \\
wiki & 0 & wiki & 0 \\
& & & \\
follow-up contact & no of hits & follow-up contact & no of hits \\
email & 79 & email & 62 \\
phone & 59 & phone & 38 \\
meeting & 32 & meeting & 24 \\
workshop/seminar/conf & 9 & workshop/seminar/conf & 3 \\
video-conferencing & 2 & video-conferencing & 0 \\
blog & 0 & blog & 0 \\
online discussion forum & 0 & online discussion forum & 0 \\
wiki & 0 & wiki & 0
\end{tabular}




\section{$\underline{\text { Appendix 30: Case } 2 \text { survey results - media/ICT use by tie strength }}$}

Media/ICT use differentiated by strong ties and weak ties and the three phases of initial contact, follow-up contact and maintenance.

\begin{tabular}{|c|c|c|c|}
\hline \multicolumn{2}{|l|}{ Strong ties } & \multicolumn{2}{|l|}{ Weak ties } \\
\hline initial contact & no of hits & initial contact & no of hits \\
\hline email & 200 & email & 27 \\
\hline phone & 95 & phone & 10 \\
\hline meeting & 49 & meeting & 6 \\
\hline workshop/seminar/conf & 51 & workshop/seminar & 5 \\
\hline video-conferencing & 2 & video-conferencing & 1 \\
\hline online discussion forum & 2 & online discussion forum & 0 \\
\hline blog & 1 & blog & 0 \\
\hline wiki & 0 & wiki & 0 \\
\hline casual & 3 & no casual & \\
\hline follow-up contact & no of hits & follow-up contact & no of hits \\
\hline email & 184 & email & 18 \\
\hline phone & 123 & phone & 7 \\
\hline meeting & 97 & meeting & 15 \\
\hline workshop/seminar & 18 & workshop/seminar & 1 \\
\hline video-conferencing & 2 & video-conferencing & 0 \\
\hline other:face-to-face casual & 1 & blog & 0 \\
\hline blog & 0 & online discussion forum & 1 \\
\hline online discussion forum & 0 & wiki & 0 \\
\hline wiki & 0 & & \\
\hline maintenance & no of hits & maintenance & no of hits \\
\hline email & 216 & email & 23 \\
\hline phone & 125 & phone & 11 \\
\hline meeting & 57 & meeting & 1 \\
\hline workshop/seminar & 10 & workshop/seminar & 2 \\
\hline online discussion forum & 3 & online discussion forum & 1 \\
\hline other: casual & 2 & video-conferencing & 0 \\
\hline video-conferencing & 1 & blog & 0 \\
\hline blog & 0 & wiki & 0 \\
\hline wiki & 0 & & \\
\hline
\end{tabular}




\section{$\underline{\text { Appendix 31: Case } 3 \text { survey results - media/ICT use by tie strength }}$}

Media/ICT use differentiated by strong ties and weak ties and the three phases of initial contact, follow-up contact and maintenance.

$\begin{array}{llll}\text { Strongly tied } & \text { Weakly tied } & \\ \text { initial contact } & \text { no of hits } & \text { initial contact } & \text { no of hits } \\ \text { email } & 113 & \text { email } & 18 \\ \text { phone } & 82 & \text { phone } & 12 \\ \text { meeting } & 59 & \text { meeting } & 0 \\ \text { workshop/seminar } & 50 & \text { workshop/seminar } & 3 \\ \text { video-conferencing } & 1 & \text { video-conferencing } & 0 \\ \text { online discussion forum } & 3 & \text { online discussion forum } & 0 \\ \text { blog } & 0 & \text { blog } & 0 \\ \text { wiki } & 0 & \text { wiki } & 0 \\ & & & \\ \text { follow-up contact } & \text { no of hits } & \text { follow-up contact } & \text { no of hits } \\ \text { email } & 154 & \text { email } & 17 \\ \text { phone } & 93 & \text { phone } & 11 \\ \text { meeting } & 57 & \text { meeting } & 6 \\ \text { workshop/seminar } & 8 & \text { workshop/seminar } & 0 \\ \text { video-conferencing } & 1 & \text { video-conferencing } & 0 \\ \text { online discussion forum } & 2 & \text { online discussion forum } & 0 \\ \text { blog } & 6 & \text { blog } & 0 \\ \text { wiki } & 0 & \text { wiki } & 0 \\ & & & \\ \text { maintenance } & \text { no of hits } & \text { maintenance } & \text { no of hits } \\ \text { email } & 165 & \text { email } & 16 \\ \text { phone } & 86 & \text { phone } & 12 \\ \text { meeting } & 31 & \text { meeting } & 2 \\ \text { workshop/seminar } & 9 & \text { workshop/seminar } & 0 \\ \text { online discussion forum } & 1 & \text { online discussion forum } & 0 \\ \text { video-conferencing } & 1 & \text { video-conferencing } & 0 \\ \text { blog } & 2 & \text { blog } & 0 \\ \text { wiki } & 0 & \text { wiki } & 0\end{array}$


Appendix 32: Case 1 survey results - preferred media characteristics

Media characteristic preferences for strongly tied givers of advice

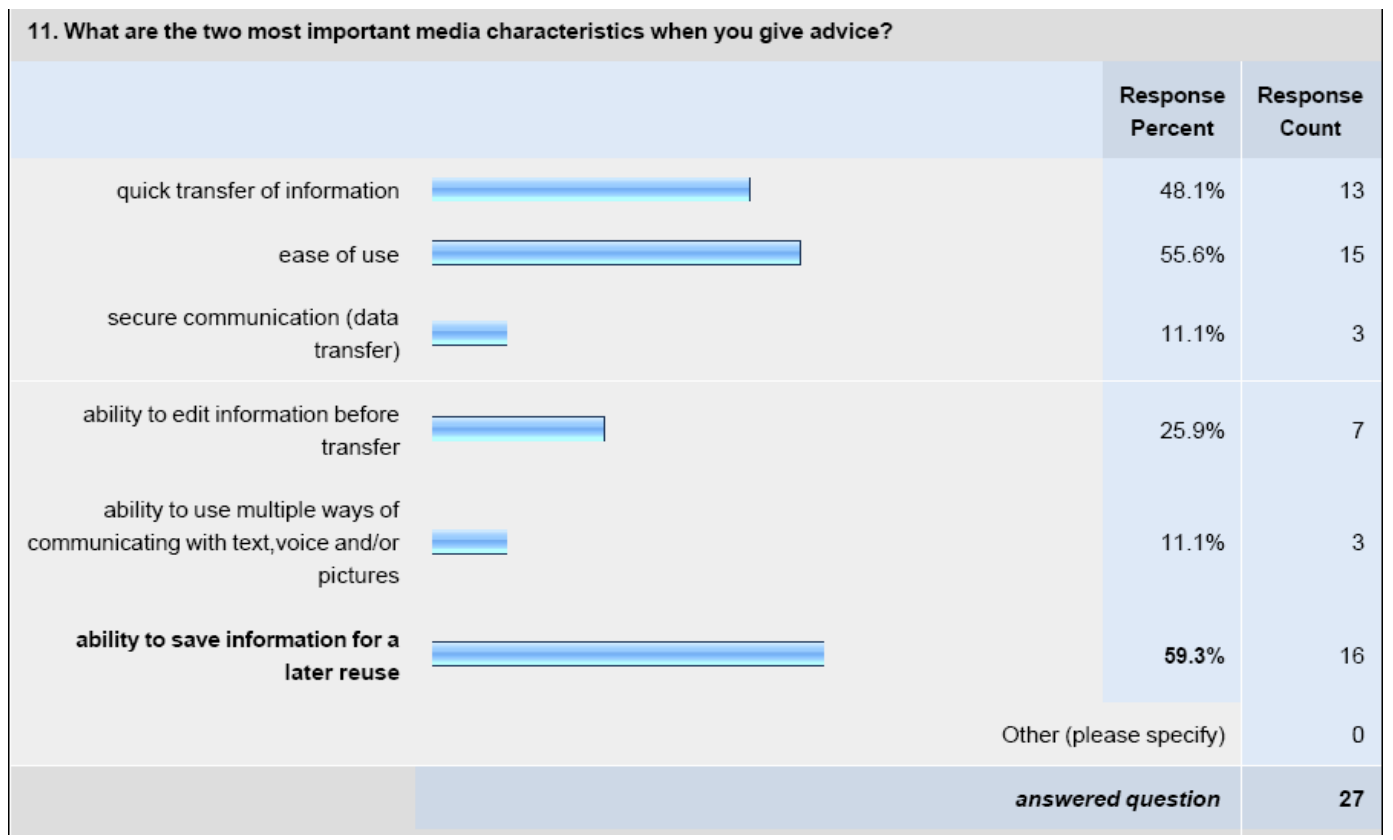

Media characteristic preferences for strongly tied seekers of advice

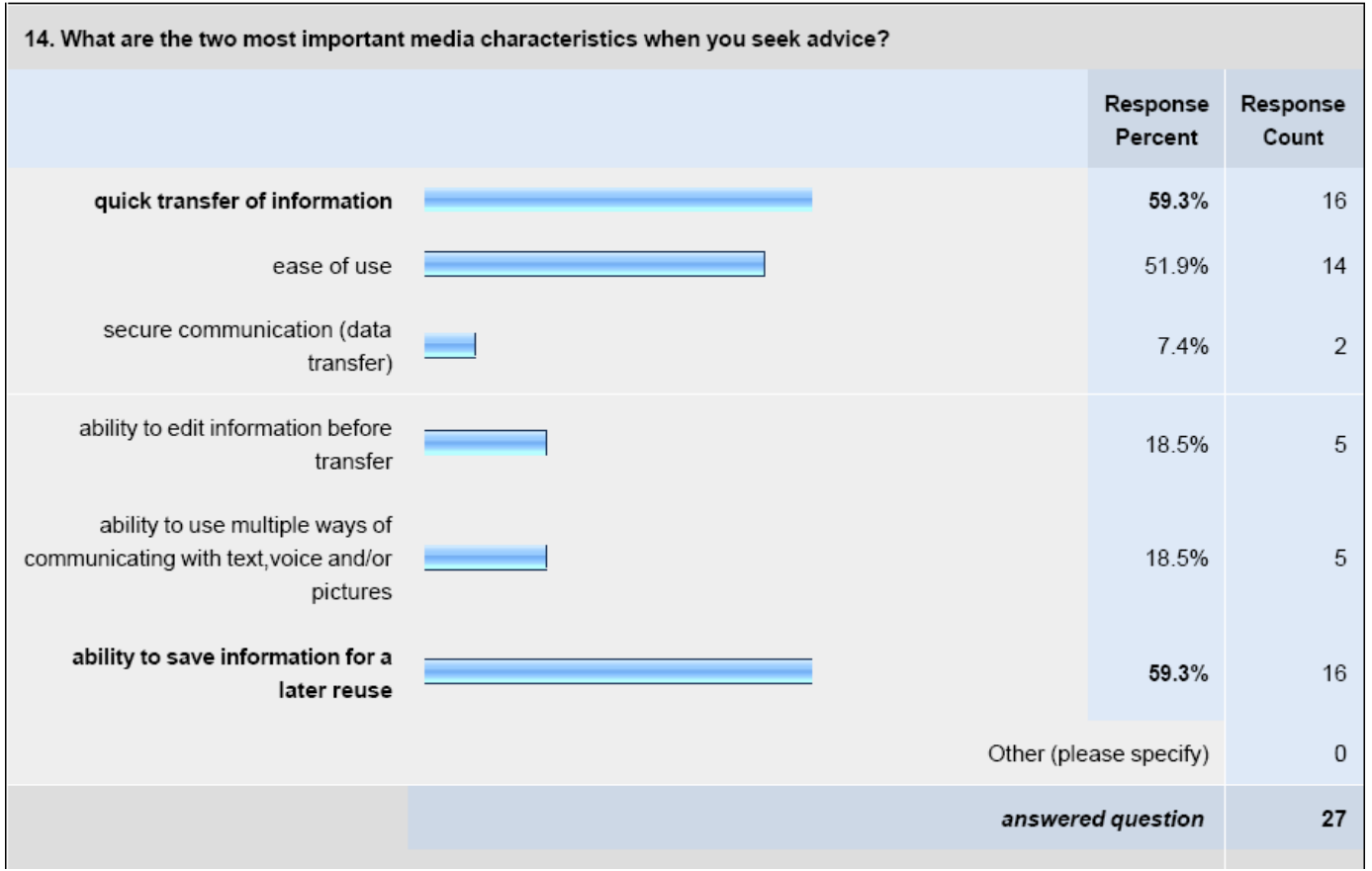




\section{Media characteristic preferences for weakly tied givers of advice}

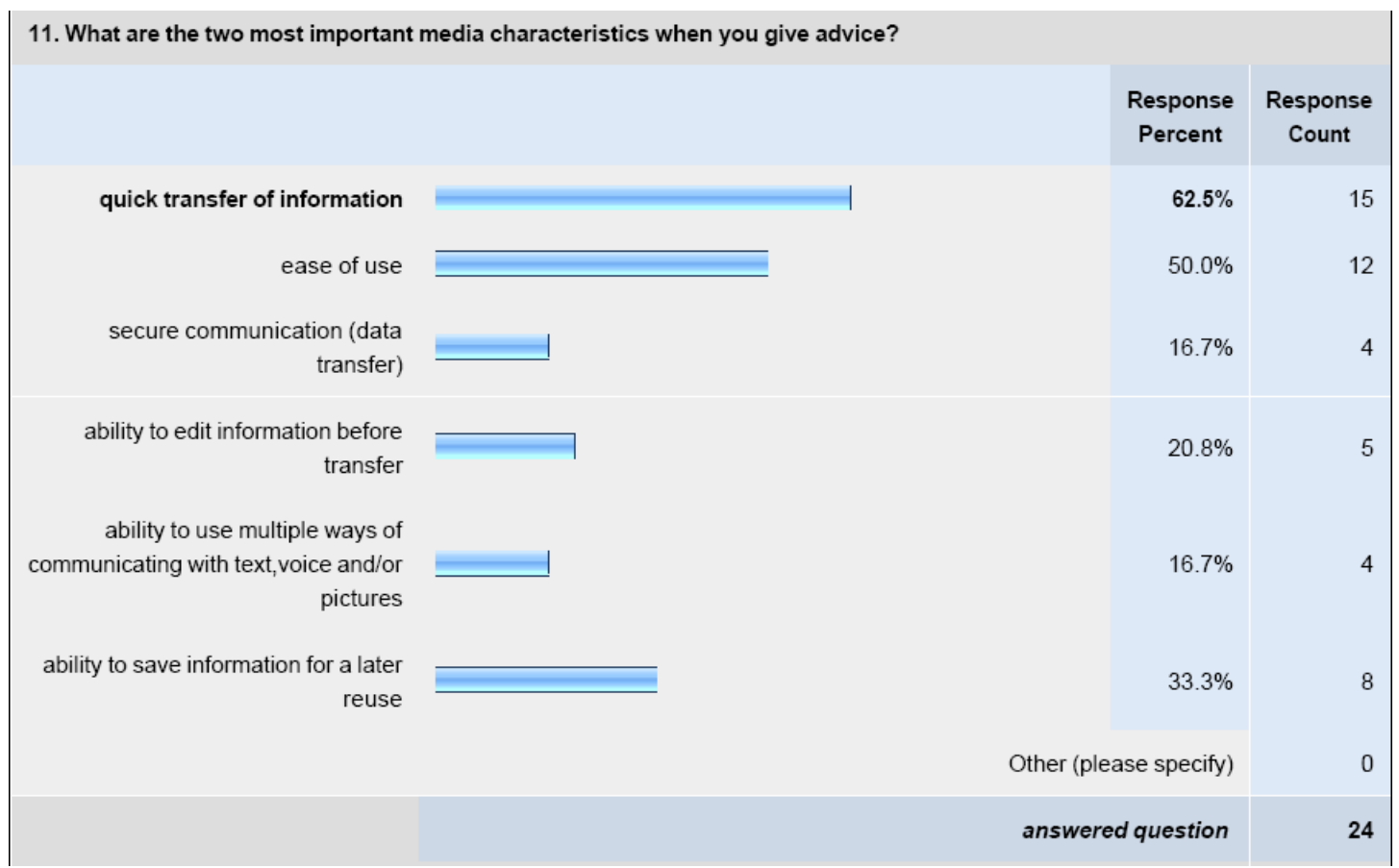

\section{Media characteristic preferences for weakly tied seekers of advice}

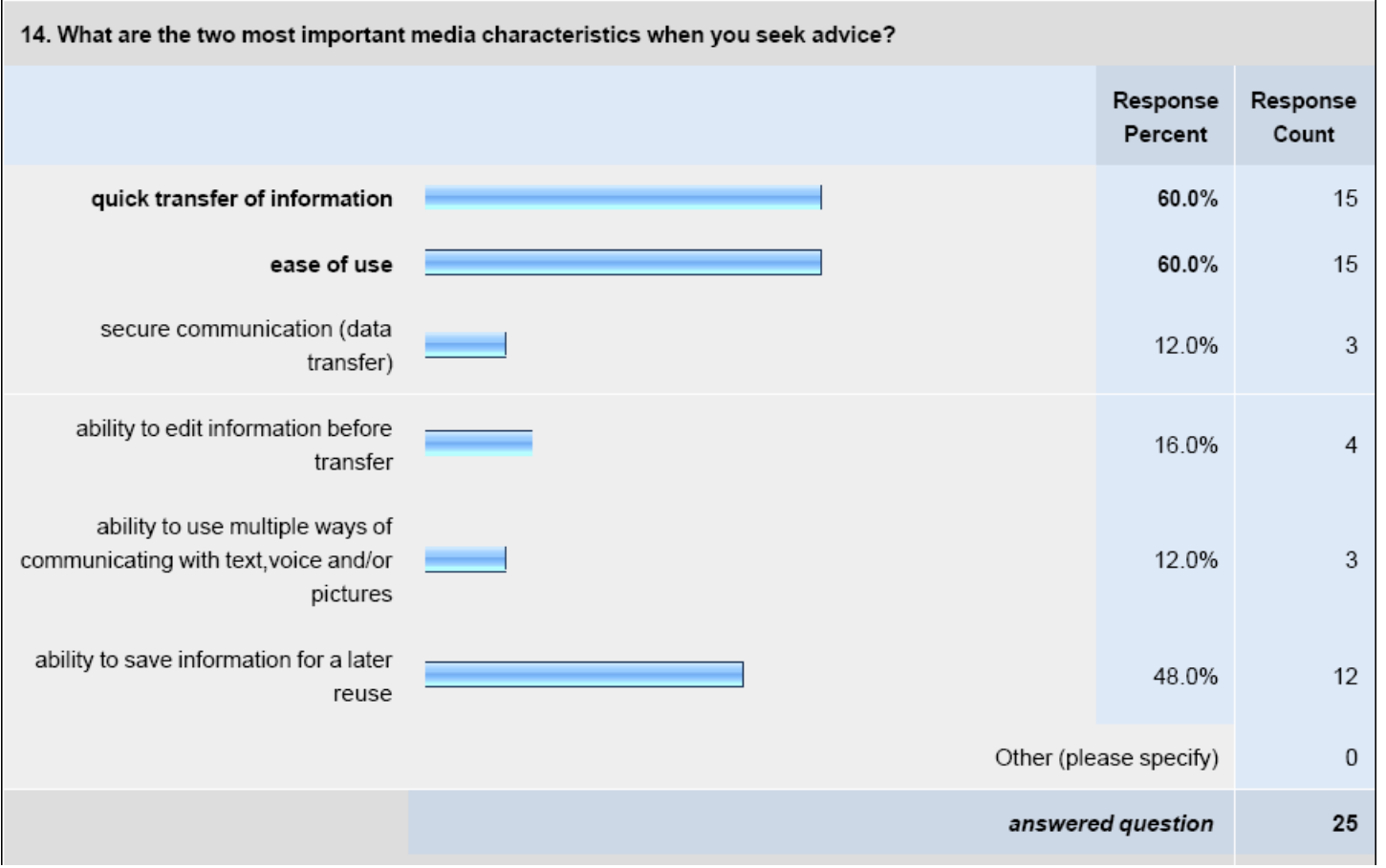




\section{$\underline{\text { Appendix 33: Case } 2 \text { survey results - preferred media characteristics }}$}

\section{Media characteristic preferences for strongly tied givers of advice}

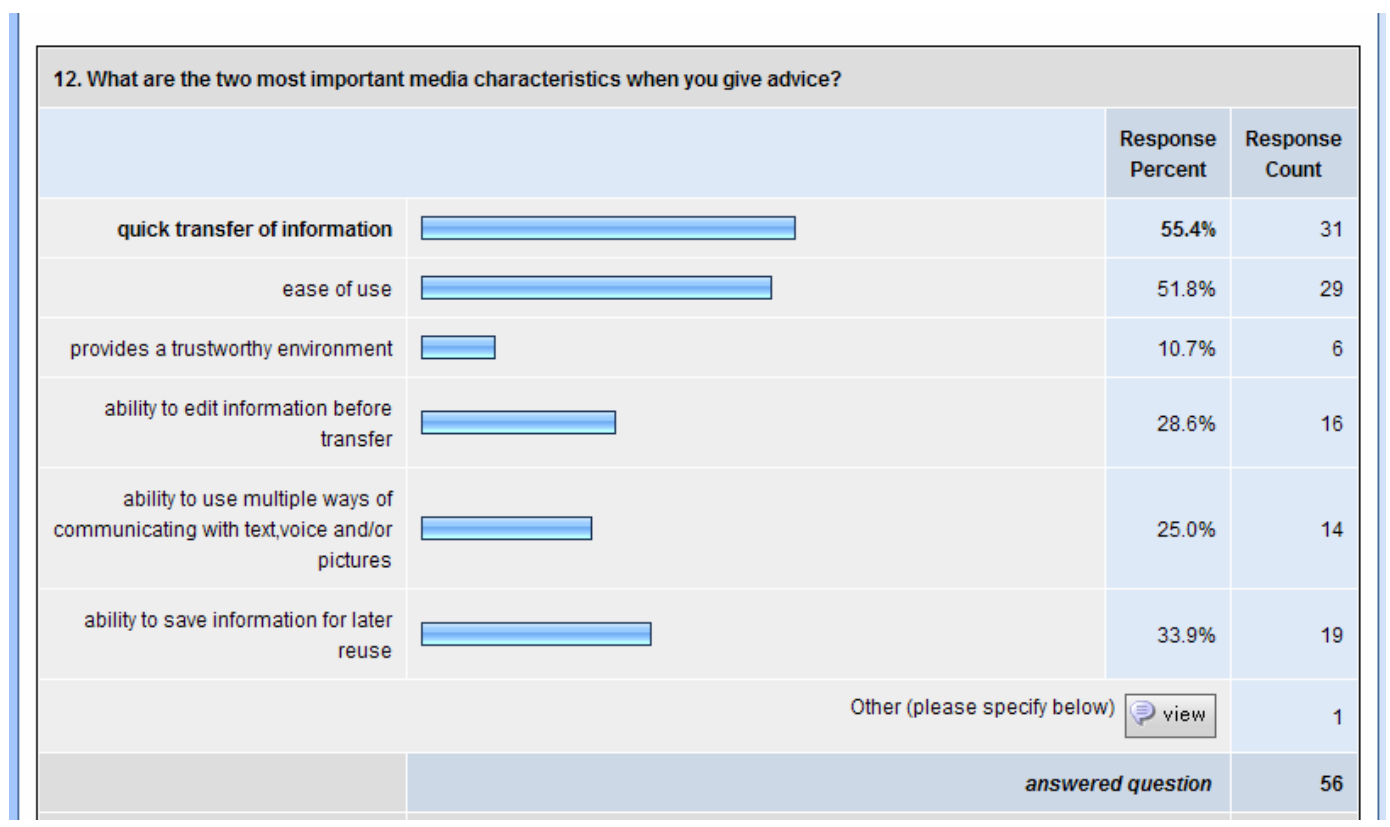

\section{Media characteristic preferences for strongly tied seekers of advice}

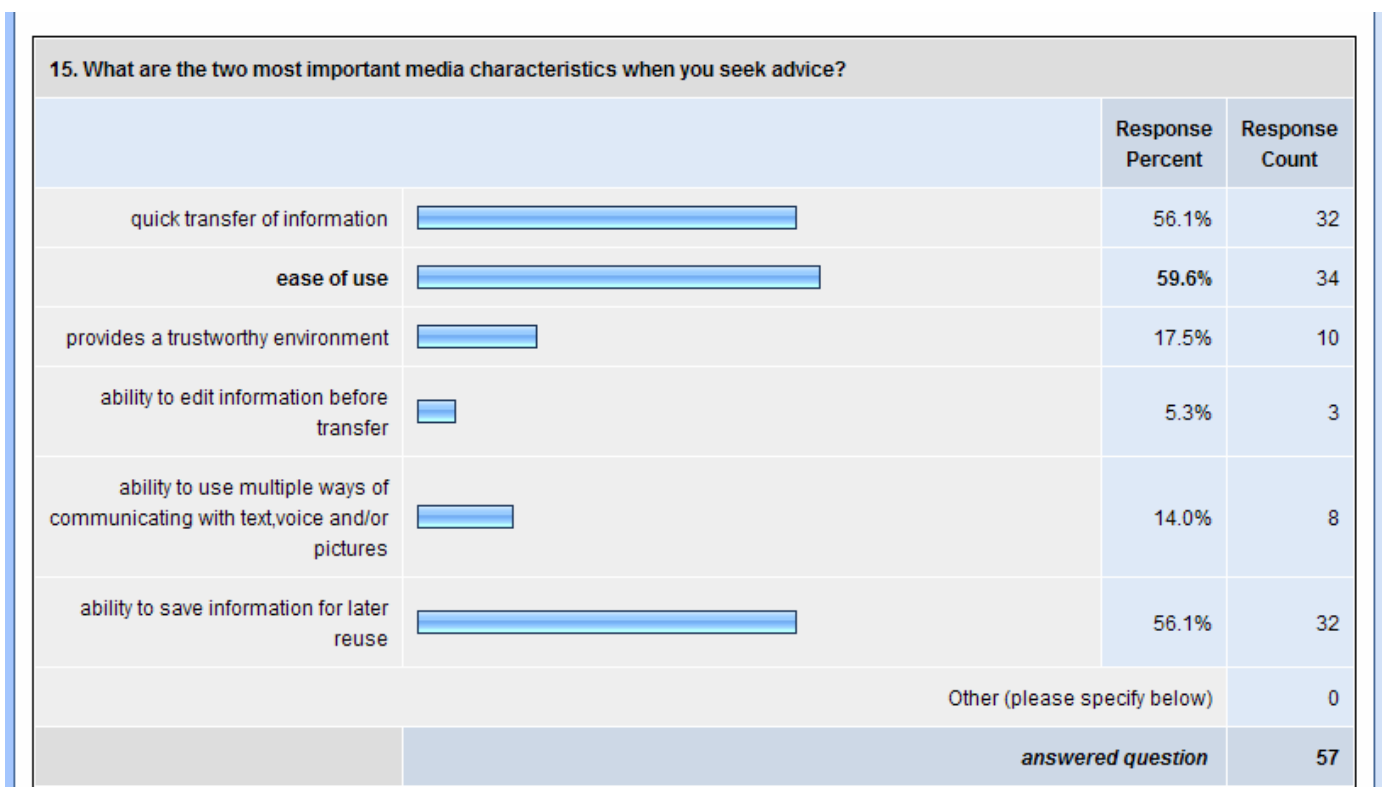




\section{Media characteristic preferences for weakly tied givers of advice}

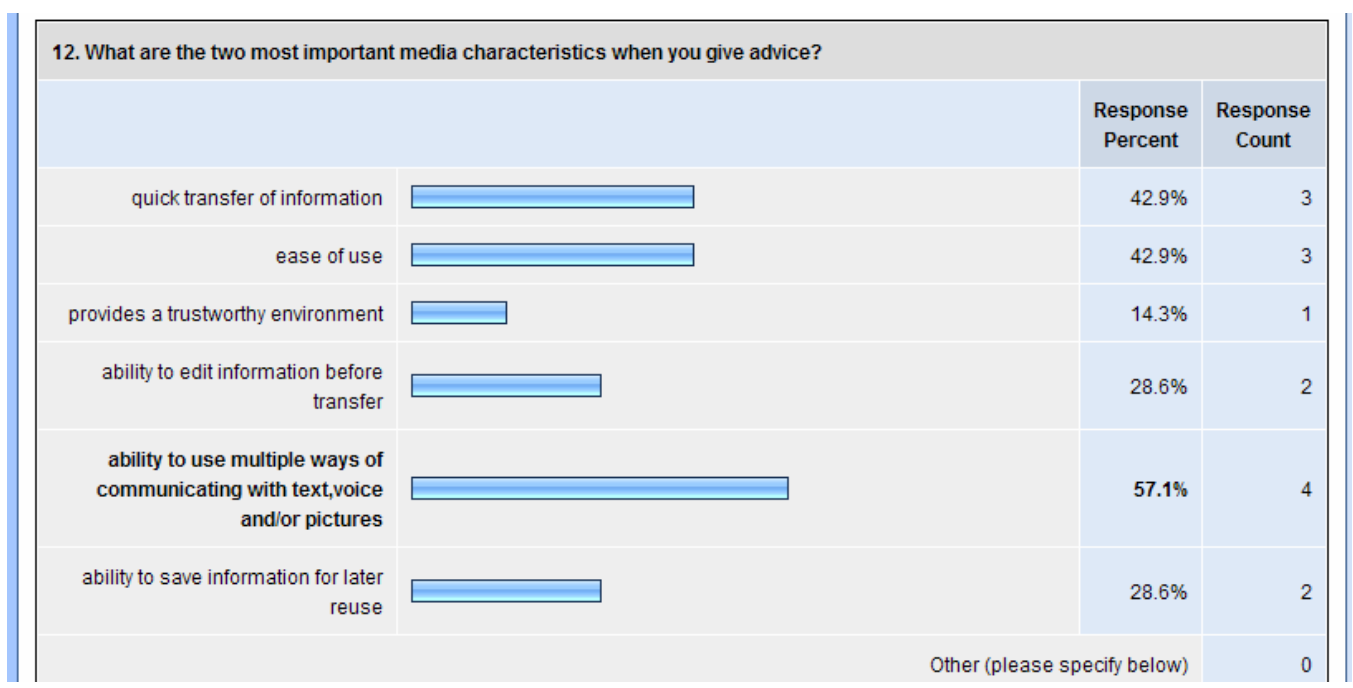

\section{Media characteristic preferences for weakly tied seekers of advice}

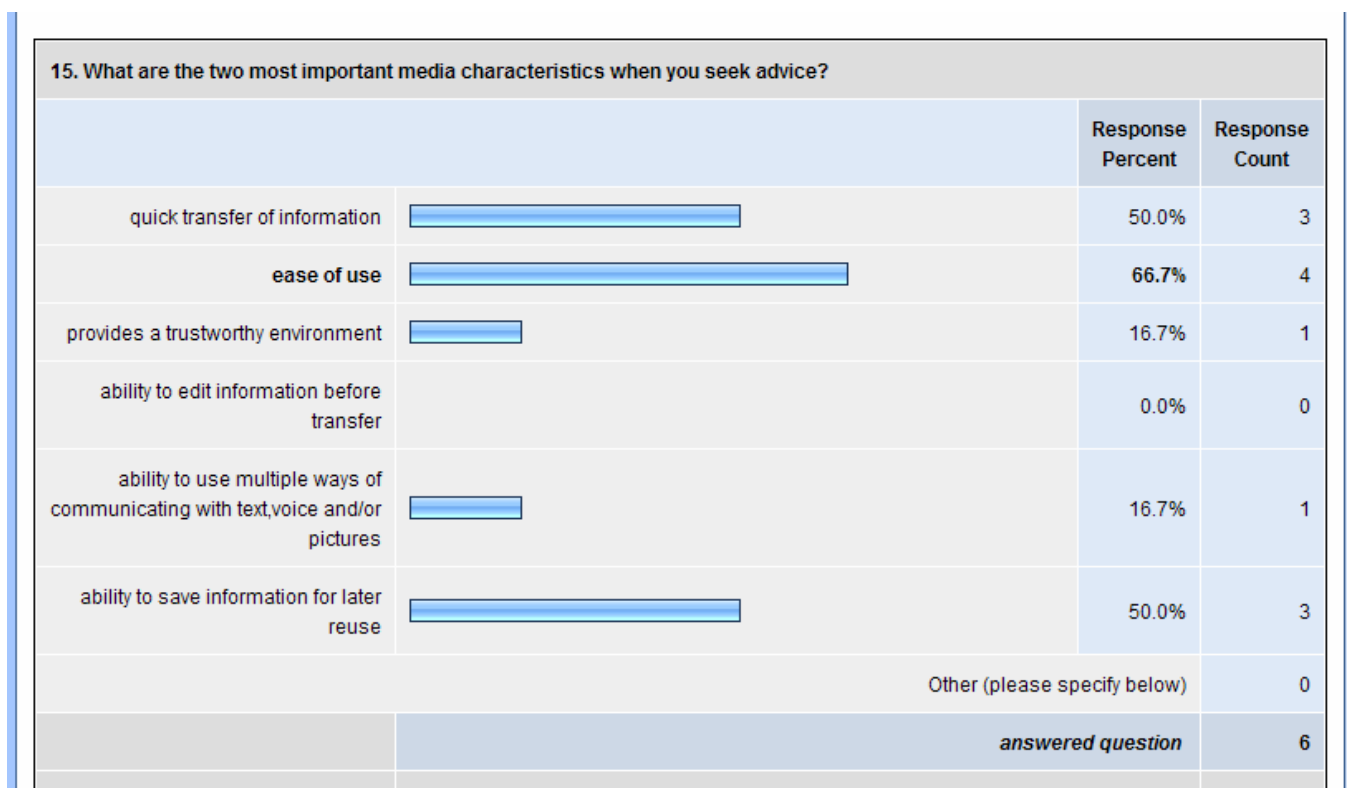




\section{$\underline{\text { Appendix 34: Case } 3 \text { survey results - preferred media characteristics }}$}

\section{Media characteristic preferences for strongly tied givers of advice}

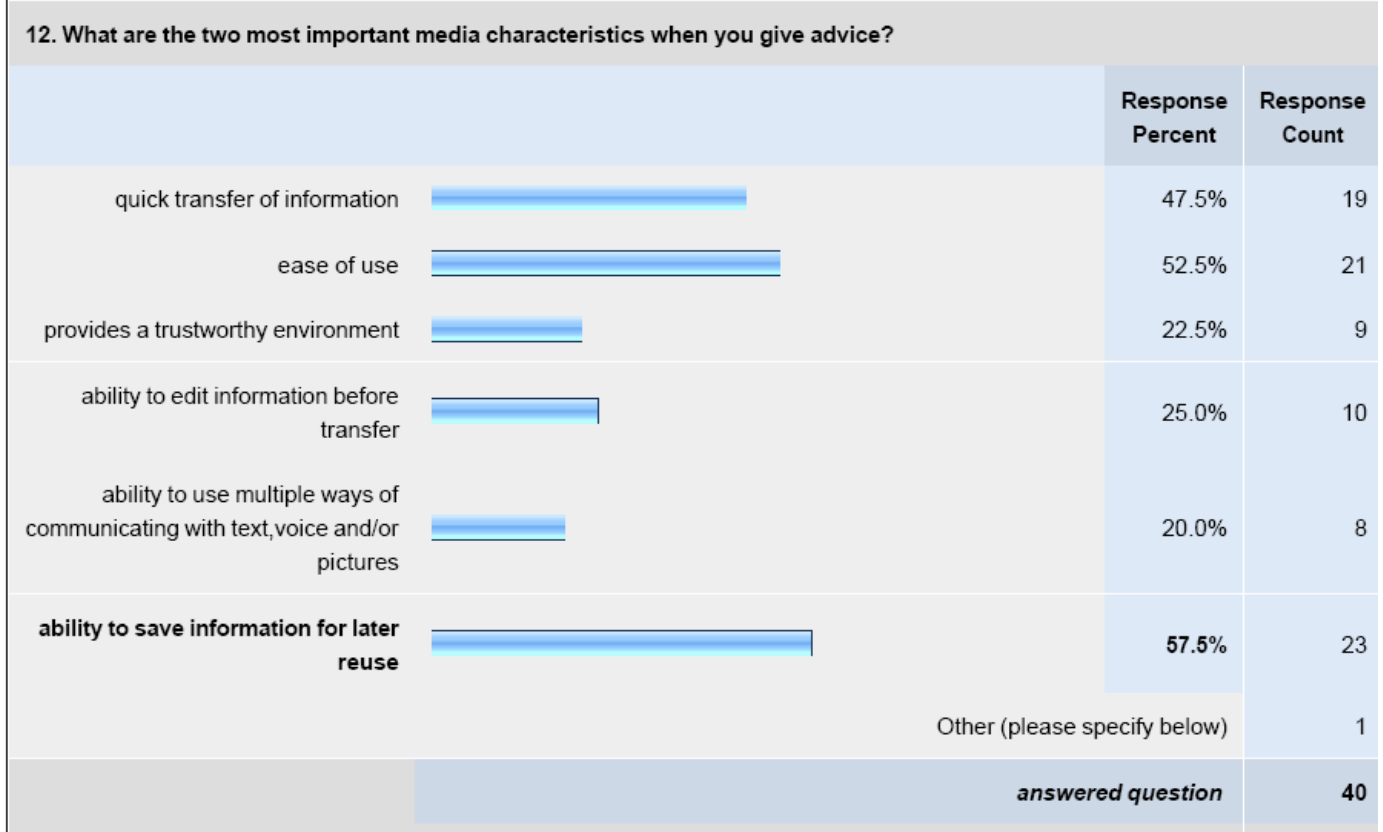

Media characteristic preferences for strongly tied seekers of advice

15. What are the two most important media characteristics when you seek advice?

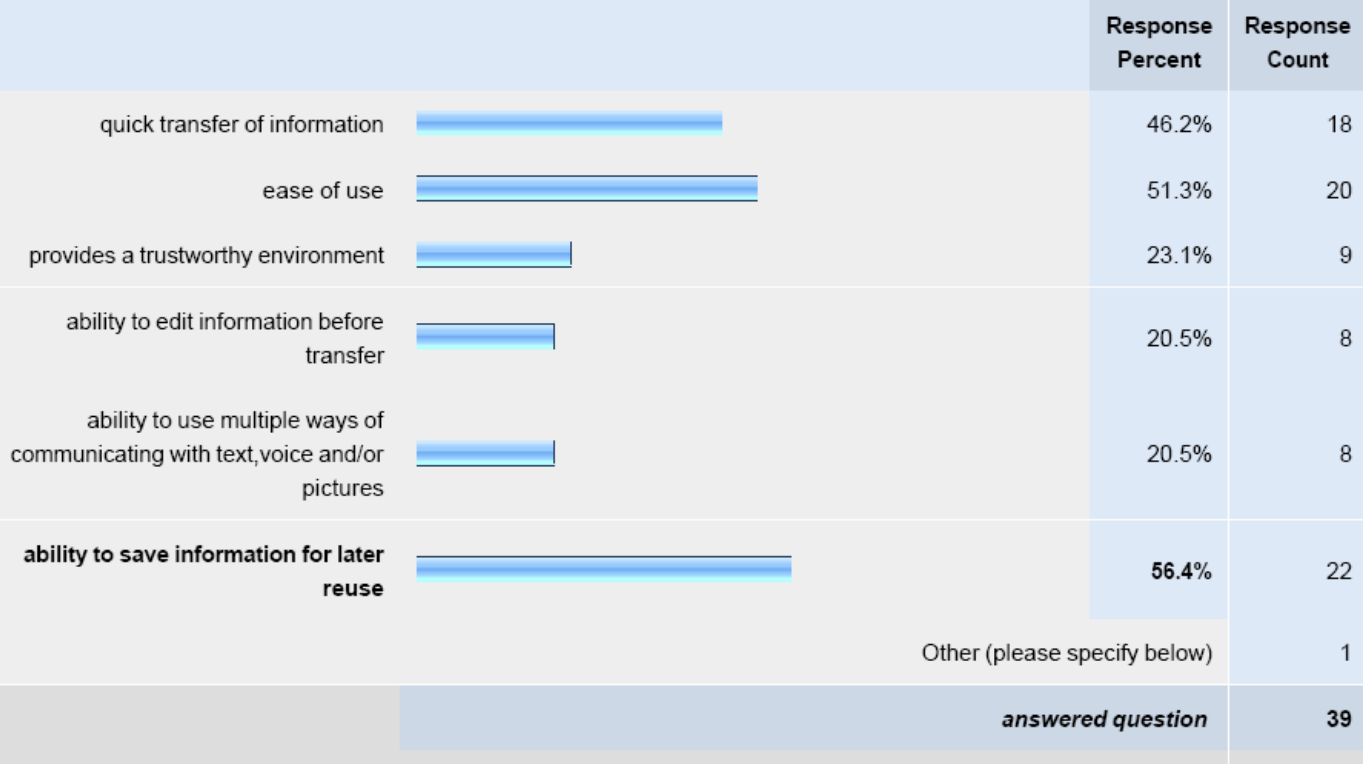




\section{Media characteristic preferences for weakly tied givers of advice}

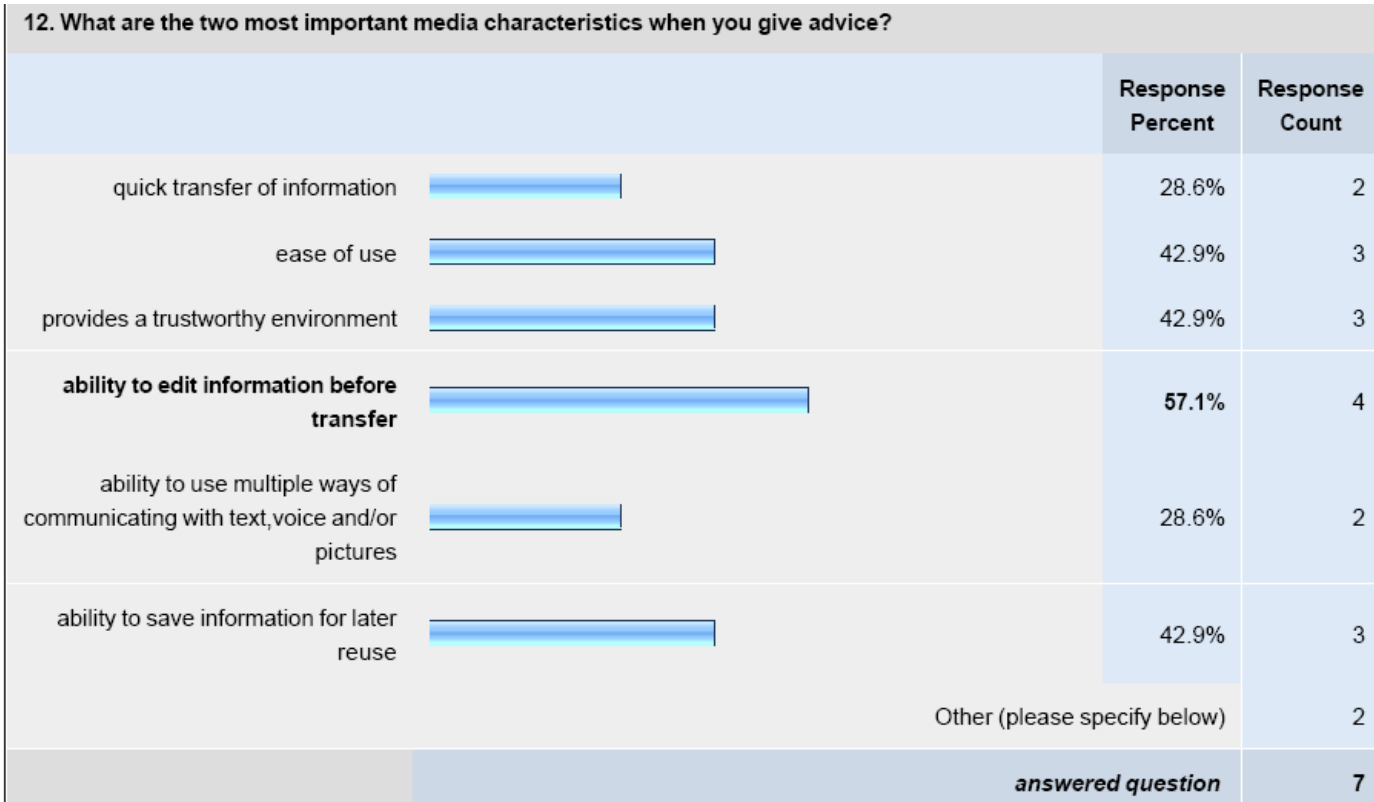

\section{Media characteristic preferences for weakly tied seekers of advice}

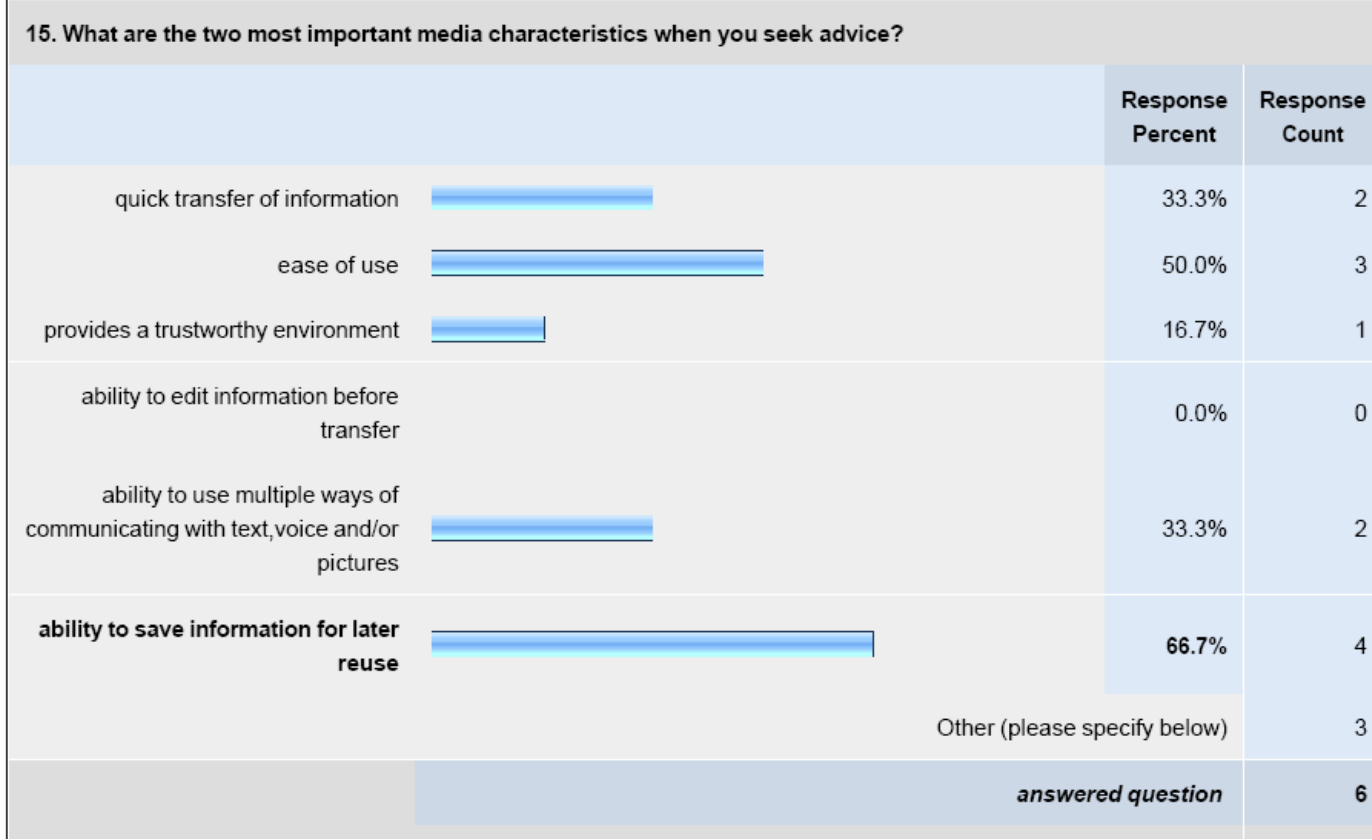




\section{Appendix 35: Case 1 survey results - preferred knowledge services}

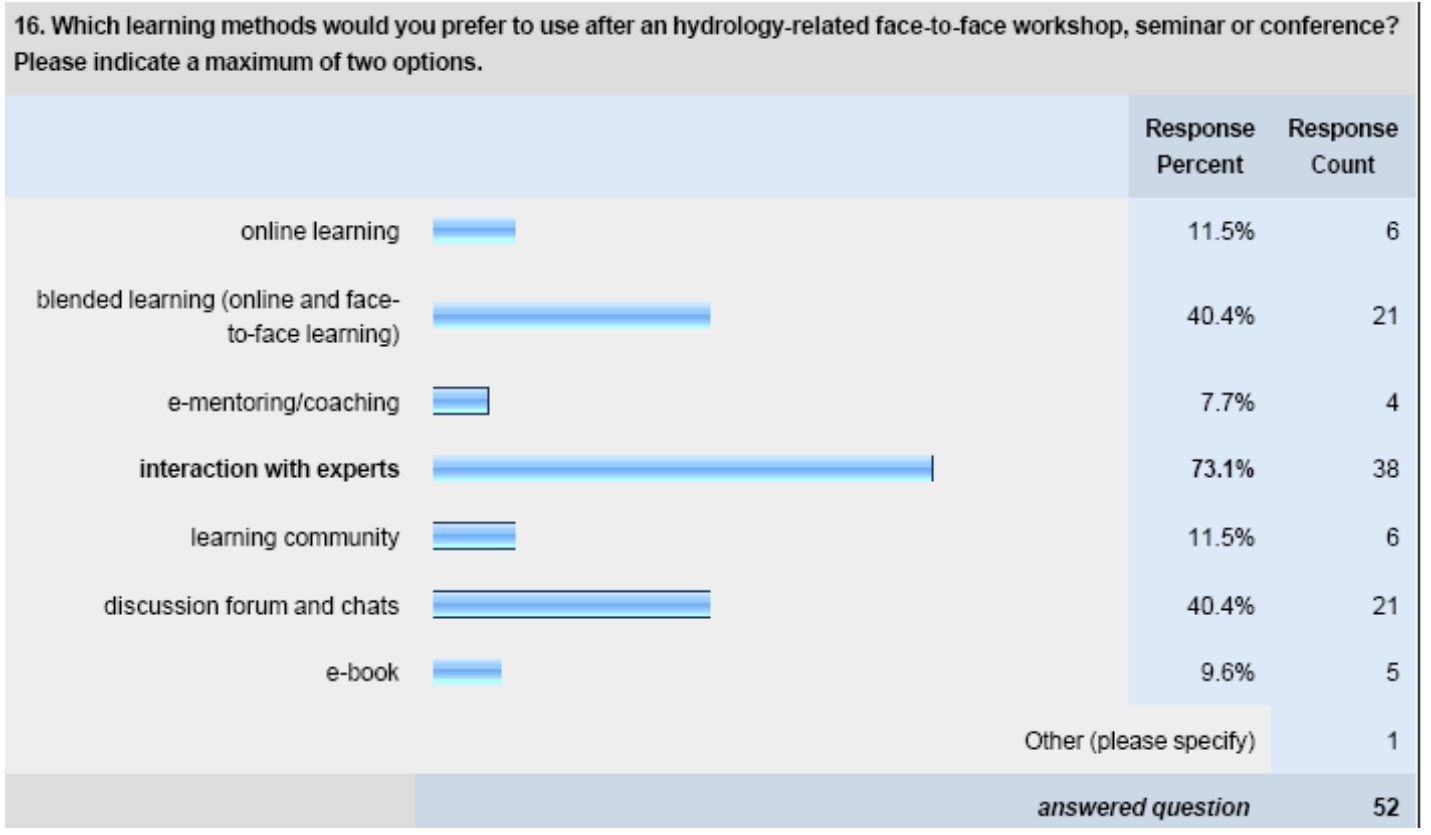

\section{By gender and throughout all job experience levels}

\begin{tabular}{|c|c|c|c|}
\hline \multicolumn{4}{|l|}{ 2. Your gender? } \\
\hline & & $\begin{array}{c}\text { Response } \\
\text { Percent }\end{array}$ & $\begin{array}{c}\text { Response } \\
\text { Count }\end{array}$ \\
\hline Male & 1 & $92.1 \%$ & 35 \\
\hline Female & $\Longrightarrow$ & $7.9 \%$ & 3 \\
\hline \multirow{2}{*}{\multicolumn{4}{|c|}{ 4. How many years have you been working in this or a similar job position? }} \\
\hline & & & \\
\hline & & $\begin{array}{l}\text { Response } \\
\text { Percent }\end{array}$ & $\begin{array}{c}\text { Response } \\
\text { Count }\end{array}$ \\
\hline$<1$ year & $\Xi$ & $5.3 \%$ & 2 \\
\hline $1-3$ years & 1 & $13.2 \%$ & 5 \\
\hline 4-10 years & 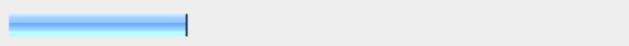 & $26.3 \%$ & 10 \\
\hline $11-19$ years & $\overline{\overline{ }}$ & $15.8 \%$ & 6 \\
\hline 20-30 years & 1 & $26.3 \%$ & 10 \\
\hline$>30$ years & $\Longrightarrow$ & $13.2 \%$ & 5 \\
\hline \multicolumn{3}{|c|}{ answered question } & 38 \\
\hline
\end{tabular}




\section{$\underline{\text { Appendix 36: Case } 2 \text { survey results - preferred knowledge services }}$}

\section{Preferred learning method (knowledge service) : online interaction with experts}

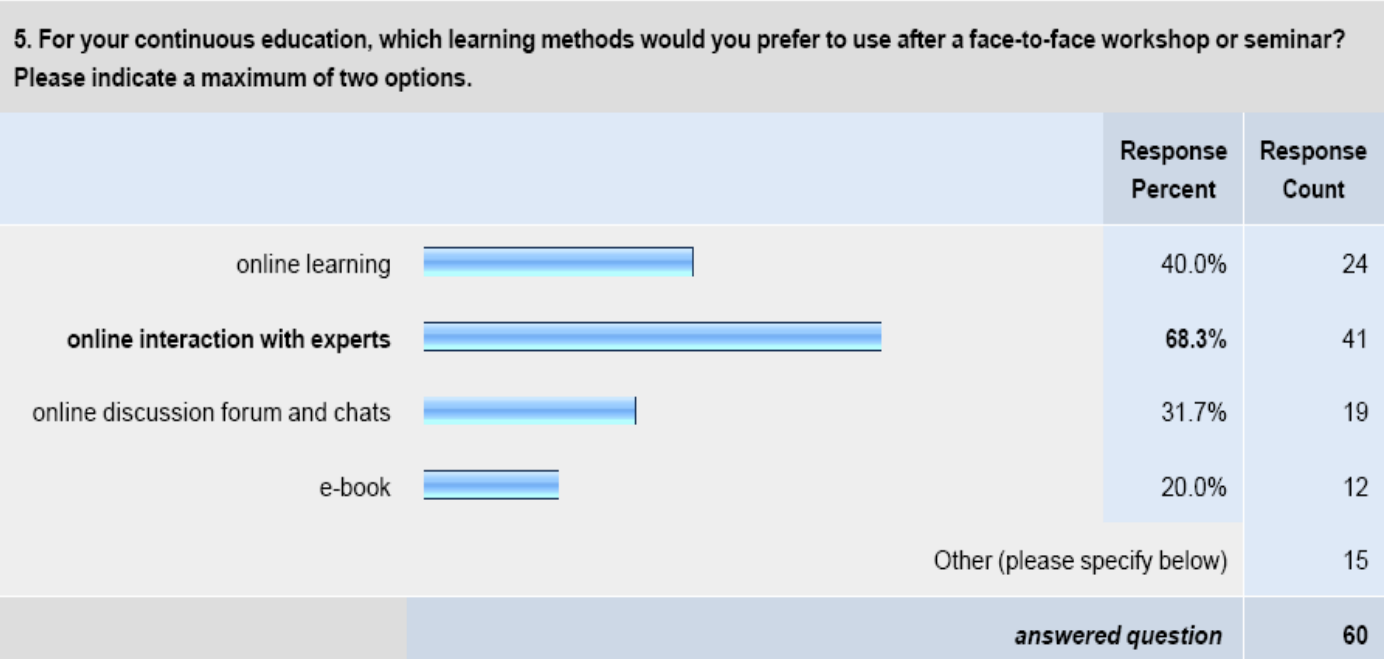

by gender and job experience

\begin{tabular}{|c|c|c|c|}
\hline \multicolumn{4}{|l|}{ 2. Your gender? } \\
\hline & & $\begin{array}{c}\text { Response } \\
\text { Percent }\end{array}$ & $\begin{array}{c}\text { Response } \\
\text { Count }\end{array}$ \\
\hline Male & 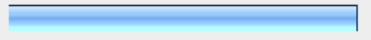 & $52.1 \%$ & 25 \\
\hline Female & $\Longrightarrow$ & $47.9 \%$ & 23 \\
\hline & & answered question & 48 \\
\hline & & skipped question & 0 \\
\hline \multicolumn{4}{|c|}{ 4. How many years have you been working in this or a similar job position? } \\
\hline & & $\begin{array}{c}\text { Response } \\
\text { Percent }\end{array}$ & $\begin{array}{c}\text { Response } \\
\text { Count }\end{array}$ \\
\hline$<1$ year & -1 & $6.3 \%$ & 3 \\
\hline $1-3$ years & $\equiv$ & $37.5 \%$ & 18 \\
\hline $4-10$ years & 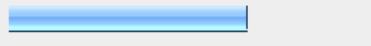 & $35.4 \%$ & 17 \\
\hline $11-19$ years & $=$ & $4.2 \%$ & 2 \\
\hline $20-30$ years & $\Longrightarrow$ & $12.5 \%$ & 6 \\
\hline$>30$ years & $=$ & $4.2 \%$ & 2 \\
\hline & & answered question & 48 \\
\hline & & skipped question & 0 \\
\hline
\end{tabular}


Appendix 37: Case 2 survey results - preferred learning method characteristics

Characteristics of knowledge services (learning methods) selected by survey participants in combination with the provided knowledge services

\section{Characteristics for online interactions with experts:}

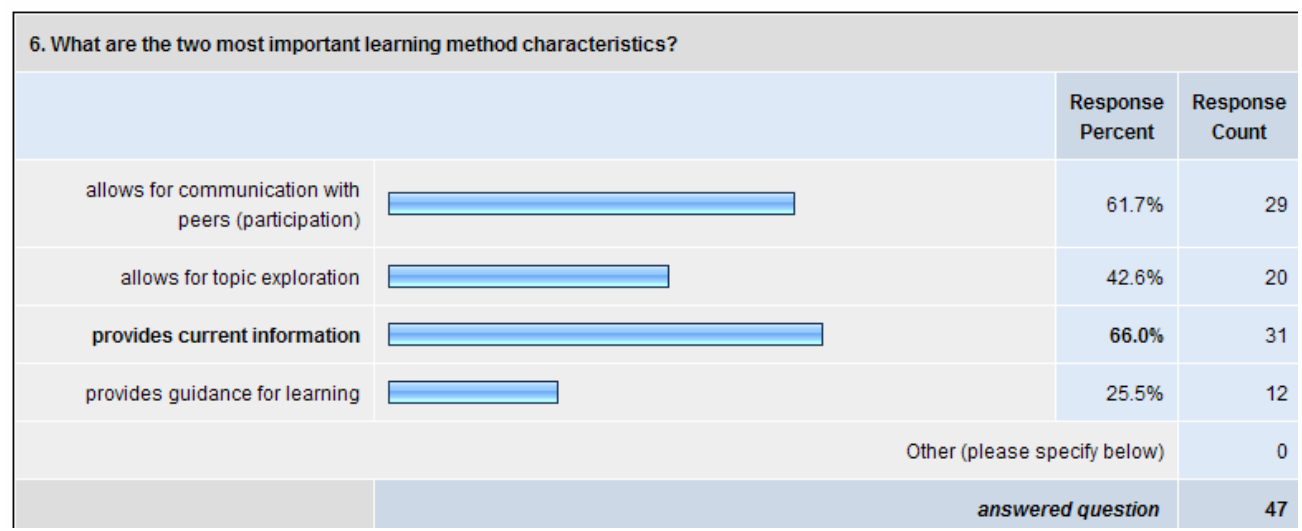




\section{Appendix 38: Case 3 survey results - preferred knowledge services}

\section{Preferred learning method (knowledge services) : online interaction with experts}

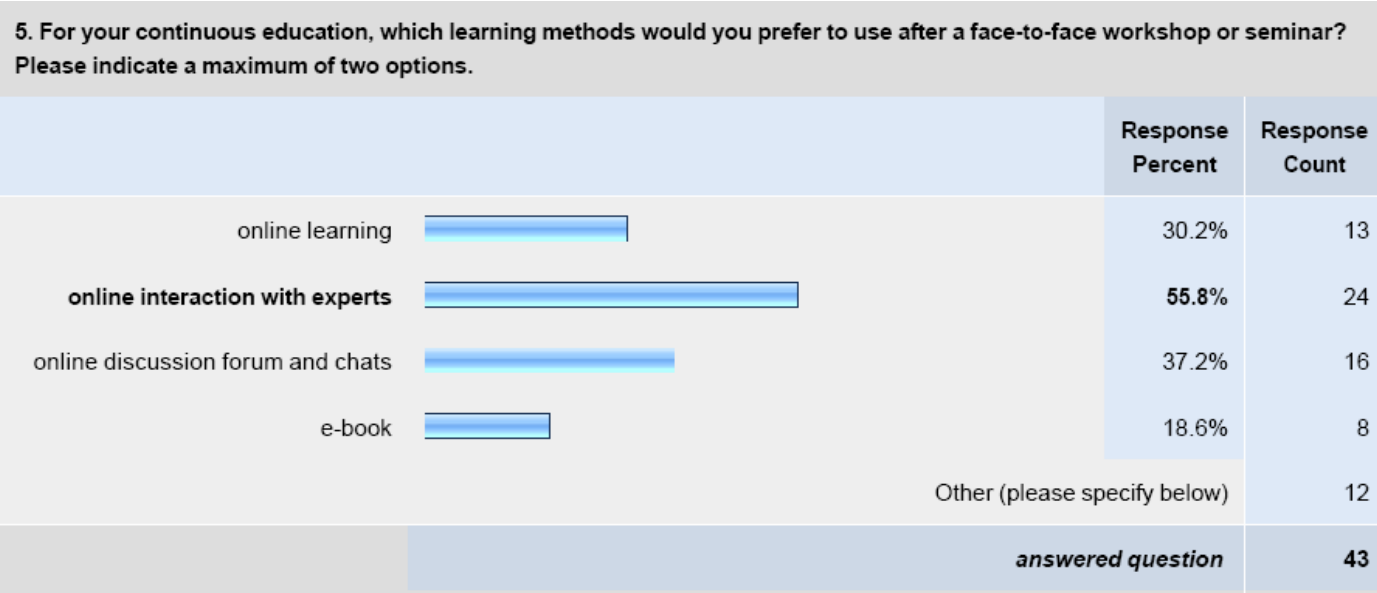

By gender and job experience

2. Your gender?

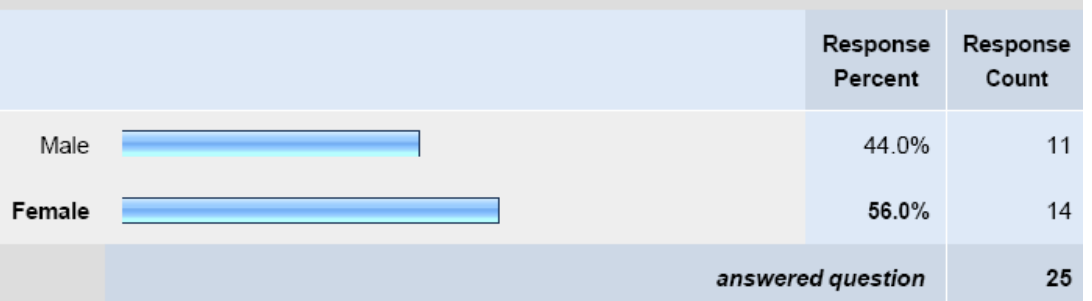

4. How many years have you been working in this or a similar job position?

\begin{tabular}{|c|c|c|c|}
\hline & & $\begin{array}{c}\text { Response } \\
\text { Percent }\end{array}$ & $\begin{array}{c}\text { Response } \\
\text { Count }\end{array}$ \\
\hline$<1$ year & 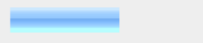 & $16.0 \%$ & 4 \\
\hline $1-3$ years & $\bar{\equiv}$ & $24.0 \%$ & 6 \\
\hline $4-10$ years & $\overline{\overline{-}}$ & $28.0 \%$ & 7 \\
\hline $11-19$ years & 9 & $8.0 \%$ & 2 \\
\hline $20-30$ years & 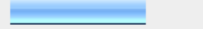 & $20.0 \%$ & 5 \\
\hline$>30$ years & 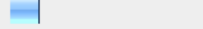 & $4.0 \%$ & 1 \\
\hline & & $d$ question & 25 \\
\hline
\end{tabular}




\section{Appendix 39: Case 3 survey results - preferred learning method characteristics}

Characteristics of knowledge services selected by survey participants in combination with the provided knowledge services (learning methods)

\section{Characteristics for online interactions with experts:}

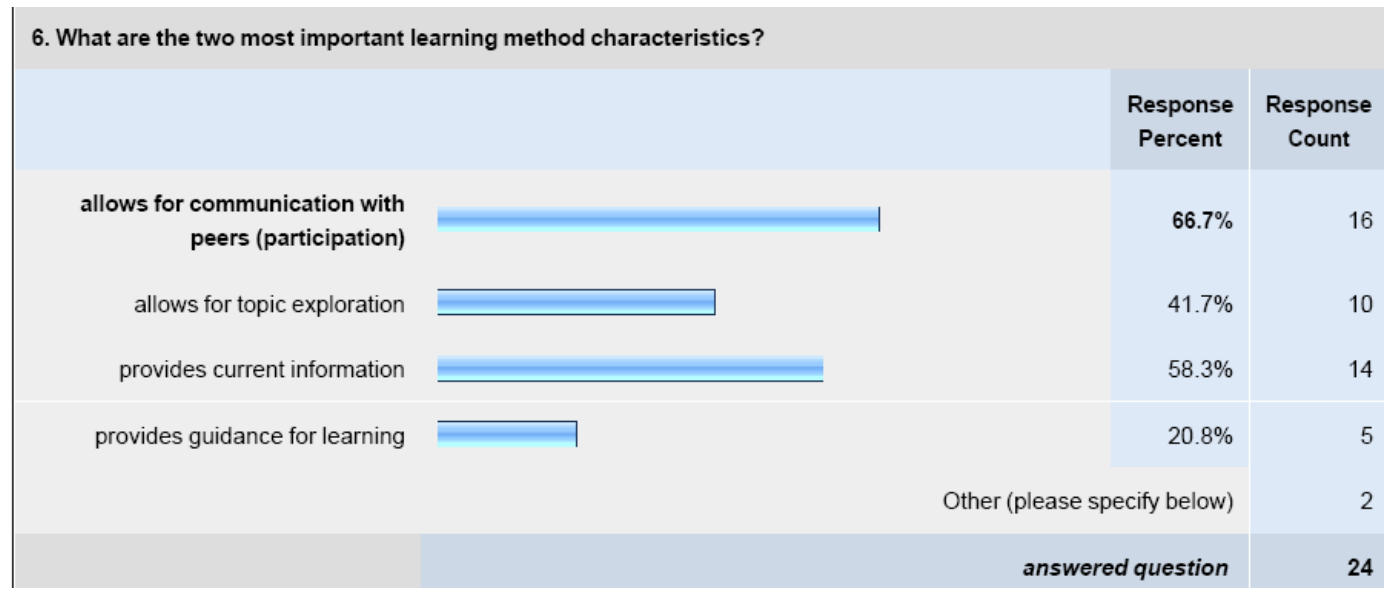




\section{Appendix 40: Interview guidelines}

\section{General work-related questions}

(1) Which organisation do you work for?

(2) Can you please describe your job?

(3) Do you work on one main topic area or with several related topic areas in your current job? Please describe the areas and projects shortly

(4) How does this area/ these areas relate to the workshop you attended?

(5) For how long have you been working in this position/ in this field?

(6) What are your tasks and responsibilities?

\section{Workshops/seminars}

Do you want to give shortly feedback on Friday's workshop?

(7) Please describe shortly any other workshops/seminars you attended in this or a similar topic area? (NIWA or somewhere else)

(8) How did you stay in contact with other participants after a workshop/seminar in order to exchange practical experiences?

(9) How would you like to stay in contact with other Hydrological Statistics workshop participants in order to exchange future work experiences with them, e. g. when working with specific statistical methods or working Tideda?

(10) Which online (learning) tools would you like to use in addition to currently provided face-to-face workshops/seminars, e.g. for exchanging information before or after workshops/seminars?

Inter-organisational communications with colleagues about work-related topics

I will ask you for some names of people you communicate with about work-related topics. I'll email your contacts then to ask them for their participation into the questionnaire of my research (Dec/Jan). Please note these names and the names of their organisations down and email them together with their email addresses to me (silke.retzer@vuw.ac.nz) after the interview. (to avoid spelling errors)

I'll also ask some questions in the interview on informal communications about during the last six months. Some examples of informal communication about work-related topics may be seeking advice from or providing advice to colleagues outside usual office hours, seek advice from/provide advice to acquaintances or 
friends you know from events such as conferences or workshops/seminars, former colleagues you previously worked together with, or colleagues who are in the same project with you, but you do not have to formally report to

Please think about the last 6 months for the following questions.

(11) Do you seek advice from or provide advice around the Hydrology topic to colleagues who are from other organisations (such as research institutes, government agencies, universities and private businesses) or from your own organisation? If yes, who are these people? ( $\sim 5$ people). Please note down their names and the names of the organisations they are working for.

(12) Why do you informally communicate with colleagues about Hydrology issues? What's happening? What do you expect to achieve? What are the outcomes of such communications?

(13) Please describe the characteristics of a helpful/useful (successful) informal work-related communication from your point of view. What are the things that are really important to you when you seek advice from/provide advice to a colleague who is working at another organisation?

(14) With around how many colleagues have you informally communicated with in relation to Hydrology issues approximately during the last 6 months? If you remember now any other names than before, please note two or three additional names down in your list (and email and the organisations they are working for).

(15) Can you please shortly describe some situations when you informally communicated with your colleagues on a Hydrological topic? What did you exchange (talking, documents)?

(16) Which tools/technologies do you use for these work-related communications (e.g. IT infrastructure, software, Web-based tools)?

(18) Which characteristics would you expect a good ICT solution should have to facilitate communications with your colleagues, especially with those from other organisations?

\section{Thank you very much for your participation!}

Please remember to email me the list of names for questions 11 and 14. I'll contact you soon with the interview's transcript for you to confirm, and with the follow-up questionnaire in about Dec/Jan. 